\title{
Schülerinnen- und Schülervorstellungen vom Grenzwertbegriff beim Ableiten
}

\author{
Dissertation \\ zur Erlangung des Grades eines Doktors der Naturwissenschaften (Dr. rer. nat.)
}

vorgelegt am Fachbereich 17 Mathematik und Informatik der Universität Gesamthochschule Paderborn

Hauke Friedrich

Paderborn, Februar 2001 


\section{Vorwort}

Die vorliegende Arbeit befasst sich mit den Vorstellungen, die Schülerinnen und Schüler von einem zentralen Begriff der Differenzialrechnung, dem Grenzwertbegriff beim Ableiten entwickeln, wenn man innen eine anregende Aufgabe zu diesem Begriff zur Verfügung stellt.

An dieser Stelle möchte ich allen danken, die mir in Gesprächen die vielen wertvollen Anregungen und kritische Hinweise gaben, die zur Entstehung dieser Arbeit unerlässlich waren. In erster Linie möchte ich meinem Doktorvater Herrn Prof. Dr. H.-D. Rinkens danken, der mir insbesondere dabei geholfen hat, den Überblick zu behalten. Ebenso gilt mein Dank Herrn Prof. Dr. P. Bender, mit dem ich so viele Details diskutieren konnte. Weiterhin möchte ich meinen Eltern Inka und Dr. Gotthard Friedrich danken, die den Grundstein für alles legten, indem sie weise meine Schullaufbahn planten und mein Studium in verschiedener Hinsicht erst ermöglichten. Zuletzt möchte ich meiner Frau Kerstin dafür danken, dass sie meine Launen während der Arbeit ertragen und mich immer unterstützt hat. 


\section{Inhalt:}

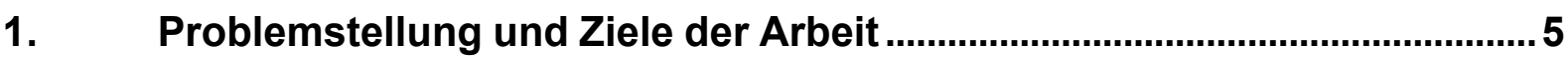

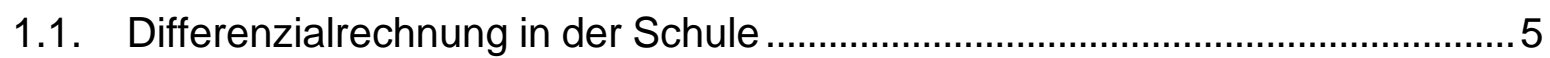

1.2. Newtons Vorstellungen von Funktionen und Differenzialrechnung .................7 Indivisibilien und das Hauptproblem ........................................................ 7

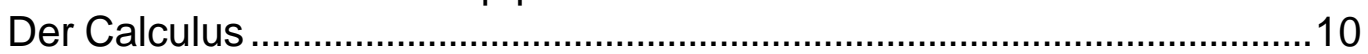

Newtons Vorstellungen vom Grenzwert ................................................11

Newtons Vorstellungen und Schulanalysis ................................................15

2. Zum Design der Untersuchung ……......................................................18

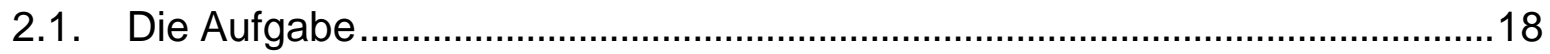

Diskussion der Teilaufgaben .................................................................19

Bezüge zur Schulpraxis .......................................................................26

2.2. Bemerkung zum Begriff der Denkwelt..........................................................27

Denkwelten und Modellbildung ..............................................................2

Die Frage nach einer Physik-Welt............................................................32

Die Frage nach einer Welt der Graphen ....................................................34

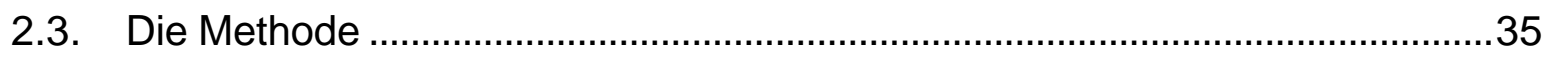

Die empirische Methode und die Stoffdidaktik .........................................36

Interpretative Forschung ........................................................................ 40

2.4. Rekrutierung der Probandinnen und Probanden.............................................44

3. Bemerkungen zum Geschwindigkeitsbegriff .........................................45

3.1. Durchschnittsgeschwindigkeit und Momentangeschwindigkeit als

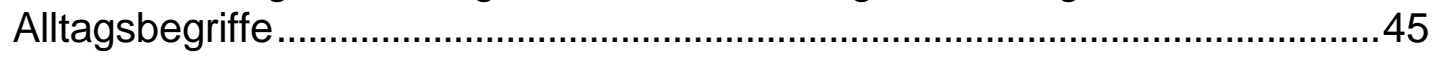

3.2. Mathematische Beschreibungen von Geschwindigkeit................................48

Weg-Zeit- oder Orts-Zeit-Funktion? ......................................................49

Die Momentangeschwindigkeit .............................................................54

Messbarkeit von Momentangeschwindigkeit...........................................60

Gibt es Momentangeschwindigkeit? - Entscheidungskriterien der

Schülerinnen und Schüler...............................................................................64

4. Die isolierende und die einbettende Sichtweise .........................................66

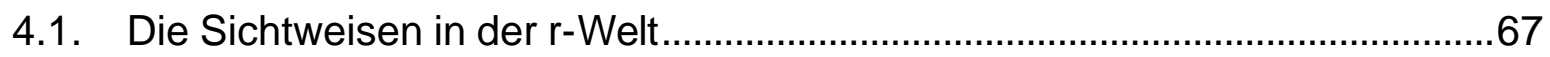

4.2. Einbettung zur Ermittlung des Grenzwerts .................................................70

4.3. Die isolierende Sichtweise ......................................................................73

4.4. Zwei Bemerkungen zu den Sichtweisen .....................................................75

Prototypische Argumentationen...........................................................75

Eine Gefahr der einbettenden Sichtweise .................................................77

4.5. Kritik an der lokalen Änderungsrate als Grundvorstellung .............................78

4.6. Statische und dynamische Sichtweise bei Folgen.......................................... 83 


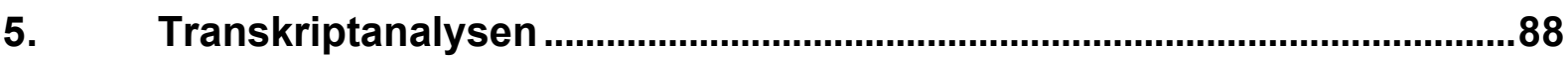

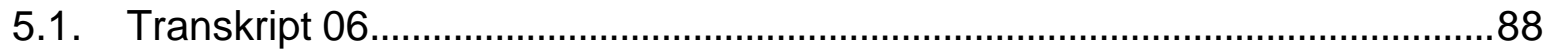

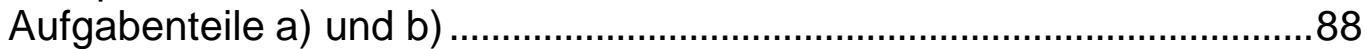

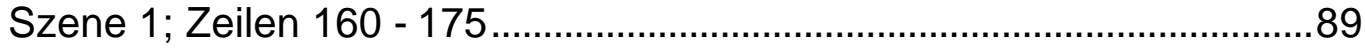

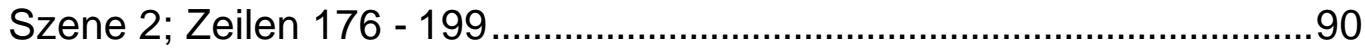

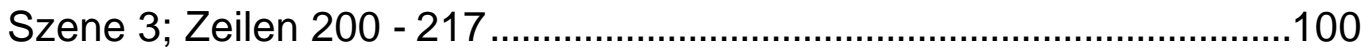

Szene 4; Zeilen 218 - 247 .................................................................103

Szene 5; Zeilen 248 - 272 .................................................................109

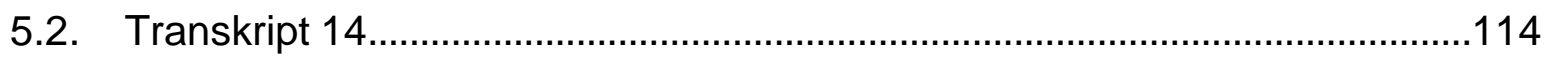

Zum Aufgabenteil a) und b) ............................................................114

Szene 1; Zeilen 25 - 45 ......................................................................115

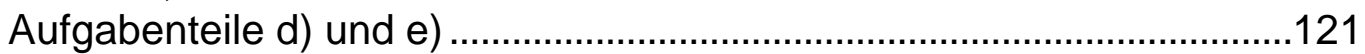

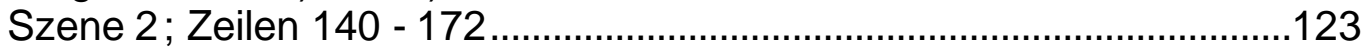

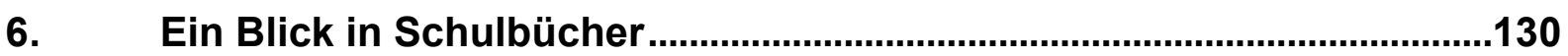

6.1. Einstiege in die Differenzialrechnung .....................................................130

6.2. Sichtweisen und Denkwelten in den Schulbüchern ......................................136

6.3. Konsequenzen für die Schulpraxis ..........................................................138

Die Sichtweisen - Ein Thema für den Unterricht? .................................138

Die Sichtweisen im Unterricht - ein Vorschlag ........................................142

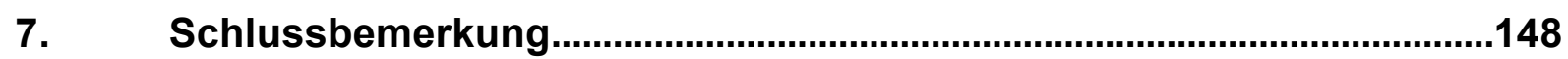

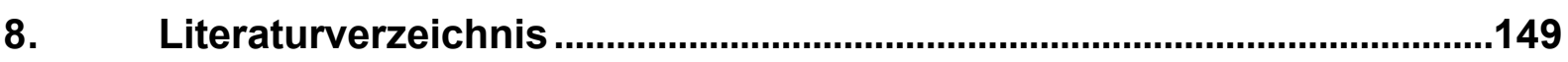

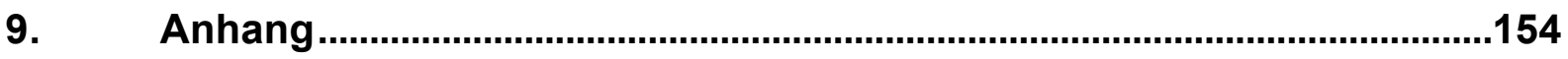

Verzeichnis der Transkripte .................................................................154

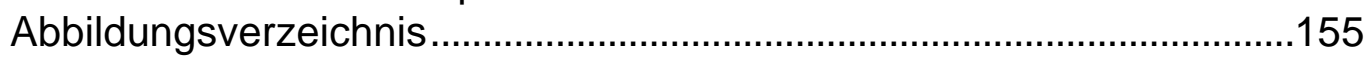

Verzeichnis der Tabellen ....................................................................155

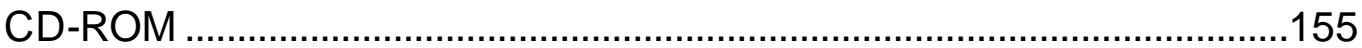




\section{Problemstellung und Ziele der Arbeit}

Mit dieser Arbeit werden vier Ziele verfolgt.

- Zum einen sollen Vorstellungen aufgedeckt werden, die Schülerinnen und Schüler vom Grenzwertbegriff beim Ableiten entwickeln.

- Weiterhin werden mögliche Ursachen für diese Vorstellungen aufgezeigt.

- Zudem soll erarbeitet werden, welche Zusammenhänge Schülerinnen und Schüler bei anwendungsorientierten Aufgaben zwischen dem Anwendungskontext in der realen Welt und den mathe matischen Methoden zur Bearbeitung sehen.

- Schließlich werden aus den Erkenntnissen Konsequenzen für den Analysisunterricht gezogen.

Oft wurde die Entwicklung der Analysis durch den Wunsch, physikalische Probleme zu lösen, vorangetrieben: Bewegungsprobleme, Schwingungs- und Pendelprobleme, aber auch Flächen und Volumenprobleme. In diesem Kapitel werden Newtons Vorstellungen von Bewegungsvorgängen vorgestellt, die ihm zur Rechtfertigung seines Ableitungskalküls dienten.

\subsection{Differenzialrechnung in der Schule}

In der Schule sollen verstärkt „anwendungsorientierte Aufgaben“ in den Unterricht einziehen. Das gilt auch und insbesondere für den Analysisunterricht, wie man z.B. dem neuen Lehrplan für die gymnasiale Oberstufe bzw. für die Gesamtschule des Landes Nordrhein-Westfalens entnehmen kann: Im Vergleich zum vorhergehenden Lehrplan ist die Sekanten-Tangentensteigungs-Vorstellung der Ableitung von der lokalen Änderungsrate abgelöst worden ${ }^{1}$. Die Sekanten-Tangenten-Vorstellung wird oft eine innermathematische genannt, während die Vorstellung der lokalen Änderungsrate fast immer mit einem außermathematischen Problem verknüpft wird. ${ }^{2}$

\footnotetext{
1 vgl. [NRW 1981] und [NRW 1999].

2 Tatsächlich ist die Vorstellung von der Tangentensteigung ein Spezialfall der Vorstellung der lokalen Änderungsrate, wie ich in 4.3 noch ausführen werde.
} 
Schon 1974 forderte Freudenthal, außermathematische Kontexte als Ausgangspunkt zur Entwicklung von Mathematik zu nutzen (und nicht umgekehrt):

\begin{abstract}
„Wenn man im traditionellen Mathematikunterricht schon die Anwendungs möglichkeiten berührt, so geschieht das immer nach dem Muster der antididaktischen Umkehrung. Statt auszugehen von der konkreten Fragestellung, um sie mathematisch zu erforschen, fängt man mit der Mathematik an, um das konkrete Problem als ,Anwendung'zu behandeln.“3
\end{abstract}

Er fordert den umgekehrten Weg: Aus Anwendungen soll Mathematik entstehen. Für die Analysis bieten sich besonders physikalische Anwendungen an. Wie bei jeder außermathematischen Einkleidung stellen die außermathematischen Begrifflichkeiten ein zusätzliches Problem dar. Daher werden für die Einführung in die Differenzialrechnung gerne Bewegungsprobleme gewählt, da die zugehörigen Begrifflichkeiten wohl als anspruchslos angesehen werden können: Da wir Menschen uns täglich zu Fuß, per Fahrrad und Auto bewegen und ständig bewegte Körper in unserer Umwelt wahrnehmen, kann man davon ausgehen, dass jede Schülerin und jeder Schüler Alltagserfahrungen aus der realen Welt (r-Welt) mitbringt. Der $r$-Welt gegenüber steht die Welt der mathematischen Beschreibungen der Bewegungsvorgänge, die mWelt ${ }^{4}$. Bewegungsaufgaben scheinen zudem besonders zur Entwicklung von Grenzwertvorstellungen der Differenzialrechnung geeignet zu sein. Schließlich hat einer der Entwickler dieses Kalküls (in einer heute nicht mehr gebräuchlichen Variante) denselben aus Vorstellungen von Bewegungsvorgängen aufgestellt. Er verfolgte das Ziel, den Zusammenhang von Weg-Zeit-Funktion und Geschwindigkeit-Zeit-Funktion mathematisch zu beschreiben. Diese Leistung Isaac Newtons soll nun kurz vorgestellt werden.

\footnotetext{
${ }^{3}$ [Freudenthal 1974], S. 126

${ }^{4}$ Zum Begriff der Welten vergleiche Kap. 2.2
} 


\title{
1.2. Newtons Vorstellungen von Funktionen und Differenzial- rechnung
}

Der Differenzialrechnung werden zwei geistige Väter zugeschrieben: Gottfried Wilhelm Leibniz (1646 - 1716) und Isaac Newton (1643 - 1727). Newton nutzte Vorstellungen von Bewe gungsvorgängen zur Entwicklung seines Grenzwertbegriffs und seiner Differenzialrechnung. Da im empirischen Teil dieser Arbeit Vorstellungen, die Schülerinnen und Schüler vom Grenzwertbegriff beim Ableiten in der Mathematik und von Momentangeschwindigkeit realer Körper die zentrale Rolle spielen, soll hier nur auf Newtons Arbeit eingegangen werden.

\section{Indivisibilien und das Hauptproblem}

Hinterlässt ein sich bewegender Punkt eine Spur, erzeugt er eine Kurve. Newton beschreibt das so:

\begin{abstract}
„Ich betrachte hier die mathematischen Größen nicht als aus äußerst kleinen Teilen bestehend, sondern als aus durch stetige Bewegung beschrieben. Linien werden beschrieben und im Beschreiben erzeugt nicht durch Aneinandersetzen von Teilen, sondern durch stetige Bewegung von Punkten; Flächen durch Bewegung von Linien; Körper durch Bewegung von Flächen; Winkel durch Rotation von Seiten; Zeiten durch stetiges Fließen; und ebenso ist es in anderen Fällen. Diese Erzeugungen finden in der Natur tatsächlich statt, und man kann sie täglich bei der Bewegung von Körpern beobachten. [... $]^{5}$
\end{abstract}

In dieser Eigenschaft nennt man den Punkt, die Strecke und die Fläche eine Indivisibilie. Durch Bewegung erzeugt sie ein geometrisches Objekt, das eine um eins höhere Dimension als sie selber besitzt. Als indivisibel, also unteilbar wird sie genau bezüglich der Dimension angesehen, die sie durch die Bewegung erschafft.

Newton bediente sich dieser Vorstellungen ausführlich bei der Entwicklung seines Verständ nisses von Funktionen. So stellt er sich vor, dass ein Funktionsgraph durch den Fluss eines gewissen Punktes (Indivisibilie) entsteht: Man nimmt zwei zu je einer Achse des Koordinatensystems parallele Geraden, die sich also (in der Regel senk-

\footnotetext{
${ }^{5}$ Newton: „Abhandlung über die Quadratur der Kurven“, 1704; nach [Becker 1975], S. 152 - 153
} 
recht) schneiden. Newton betrachtet sie als "geradweise und unbegrenzt wachsend“ 6 . Der Graph einer Funktion wird durch die Bewegung des Schnittpunktes der beiden Geraden erzeugt, vgl. Abbildung 1.

„Indem ich nun in Betracht zog, daß in gleichen Zeiten wachsende und wachsend erzeugte Größe je nach der größeren oder kleineren Geschwindigkeit, mit der sie wachsen und erzeugt werden, größer oder kleiner ausfallen, suche ich nach einer Methode zur Bestimmung der Größen aus der Geschwindigkeit der Bewegung oder des Wachsens, wodurch sie erzeugt werden. Diese Bewegungs- oder Wachstumsgeschwindigkeiten nannte ich Fluxionen, und die erzeugten Größen nannte ich Fluenten, [...].“7

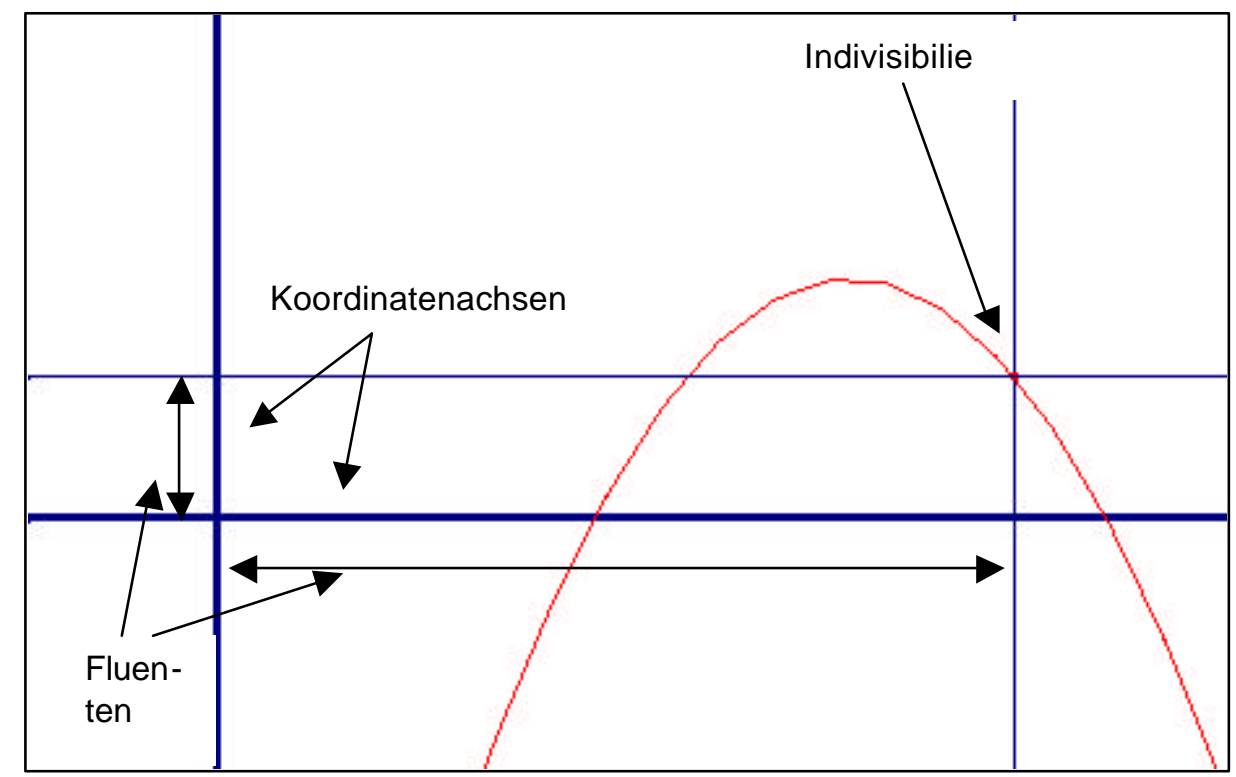

Indivisibilien, Fluenten und Fluxionen:

Die kräftigen Geraden sind die Achsen eines Koordinatensystems. Der Schnittpunkt der dünnen zu den Achsen parallelen Geraden ist die Indivisibilie, die die Kurve erzeugt.

Die Doppelpfeile deuten die Fluenten (Größen) an, die ebenfalls durch die Bewegung der Indivisibilien entstehen. Die Fluxionen der Fluenten sind die Geschwindigkeiten, mit denen sich die schmalen Linien jeweils senkrecht zu den zugehörigen Achsen bewegen.

Abbildung 1: Newtons Funktionsbegriff mit Indivisibilien, Fluenten und Fluxionen

Newton fragt hier nach einer Methode, die wir heute Integralrechnung nennen. Als er 1670/71 seine „Methodus fluxionum et serierum infinitarum“ schrieb, formuliert er mit den Hauptproblemen ebenso die Frage nach der Differenzialrechnung. Den mathe-

\footnotetext{
${ }_{7}^{6}$ [Becker 1975], S. 149 sinngemäß auch [Volkert 1988], S. 88

7 Newton: „Abhandlung über die Quadratur der Kurven“, 1704; nach [Becker 1975], S. 153
} 
matischen Zusammenhang der Lösungen beider Probleme nennen wir heute den Hauptsatz der Differenzial- und Integralrechnung :

„I. Gegeben die Länge des durchmessenen Wegs in jedem Zeitmoment. Zu finden die Geschwindigkeit der Bewegung zu einer gegebenen Zeit.

II. Wenn die Geschwindigkeit zu jeder Zeit gegeben ist, ist die Länge des beschriebenen Wegs zu finden zu einer gegebenen Zeit.“8

Das Zitat kann vermuten lassen, Newton würde ausschließlich den Zusammenhang zwischen Weg-Zeit-Funktionen und Geschwindigkeit-Zeit-Funktionen herstellen wor len. Tatsächlich aber betrachtete er eine viel allgemeinere Problemstellung:

\begin{abstract}
„Im folgenden betrachte ich die Zeit nicht als wirklich (formaliter), sondern setzte voraus, daß eine mit den anderen vorkommenden Größen gleichartige Größe mit diesen in gleichmäßigem Flusse wächst, auf welche, als gleichsam auf die Zeit, die übrigen Größen bezogen werden. Diese kann folgerichtig Zeit genannt werden. Wenn also im folgenden das Wort "Zeit“ gebraucht wird ..., ist dieses Wort nicht so zu verstehen, als ob ich die Zeit in ihrer wirklichen Bedeutung gemeint hätte, sondern in dem Sinn, daß ich jene von der Zeit verschiedene Größe im Auge habe, durch deren gleichförmiges Wachsen oder Fließen der Zeit dargestellt und gemessen wird."
\end{abstract}

Newton betrachtet allgemein gewisse gleichartige Größen, wobei eine dieser Größen stetig fließen können muss, wie die Zeit. Da die davon abhängigen Größen gleichartig zur ersten sein sollen, fließen auch sie (in Abhängigkeit von dieser). In seiner Vorstellung betrachtet Newton die erste Größe wie die Zeit, die für inn die „natürlichste" stetig fließende Größe zu sein scheint. Die Wachstumsgeschwindigkeit der abhängigen Größe entspricht nun der lokalen Änderungsrate im Allgemeinen. Man kann sich die erste Größe, Newton bezeichnet sie oft mit x, durch die Zeit parametrisiert vorstellen, $x(t)$. Bedeutet $x$ die Größe "Zeit" selber und die abhängige Größe die Weglänge, die eine bewegter Körper in der Zeit zurücklegt, entspricht die Wachstumsgeschwindigkeit der Momentangeschwindigkeit im üblichen Sinne.

\footnotetext{
${ }^{8}$ nach [Becker 1975], S. 148

9 nach [Becker 1975], S. 148 - 149
} 


\section{Der Calculus}

Der Calculus, den Newton zur Lösung seines ersten Hauptproblems verwendet, war schon zu seiner Zeit nicht neu. Fermat zum Beispiel nutzte inn schon. An der folgenden Passage kann man erkennen, wie Newton die Idee der linearen Approximation als Begründung für den Calculus benutzt:

„Die Momente der fließenden Größe (d.h. ihre unendlichen kleinen Teile, durch deren Hinzukommen in unendlich kleinen Zeitteilen die Größen selbst zugleich [jugiter] vermehrt werden) verhalten sich wie die Geschwindigkeiten, mit denen sie fließen oder wachsen.

Wenn daher das Moment der Größe $x$ dargestellt wird durch das Produkt ihrer Geschwindigkeit $\dot{x}$ mit einer unendlich kleinen Größe o (d.h. durch $\dot{x} o$ ), werden die Momente der anderen Größen $u, y, z$ durch $\dot{u} o, \dot{y} o, \dot{z} O$ darzustellen sein, weil $\dot{u} o, \dot{x} o, \dot{y} o, \dot{z} o$ zueinander dasselbe Verhältnis haben werden wie zu $\dot{u}, \dot{x}, \dot{y}, \dot{z}$. Da nun die Momente, wie z.B. $\dot{x} o$ und $\dot{y} o$, unendlich kleine Zuwüchse sind, durch die die Fluenten $x$ und $y$ in unendlich kleinen Zeitabschnitten vermehrt werden, so folgt, daß diese Größen $x$ und $y$ nach einem unendlich kleinen Zeitraum zu $x+\dot{x} o$ und $y+\dot{y} o$ werden. Die Gleichung, die die Beziehung zwischen den Fluenten zu jeder Zeit ausdrücke, wird ebensogut die Beziehung zwischen $x+\dot{x} O$ und $y+\dot{y} o$ wie die zwischen den $x$ und $y$ ausdrücken, so daß man in dieser Gleichung $x+\dot{x} o$ und $y+\dot{y} o$ für $x$ und $y$ setzen kann. Es sei demnach folgende Gleichung vorliegend“ 10

$$
y=x x \quad \text { bzw. } y-x x=0
$$

Substituiere darin $x$ durch $x+\dot{x} o$ und $y$ durch $y+\dot{y} o$ :

$$
y+\dot{y} o-(x+\dot{x} o)(x+\dot{x} o)=0 \Leftrightarrow y+\dot{y} o-x x-\dot{x} 0 \dot{x} o-2 x \dot{x} o=0
$$

Mit $y-x x=0$ bleibt:

$$
\dot{y} 0-\dot{x} \circ \dot{x} 0-2 x \dot{x} 0=0
$$

Dividiere durch 0 :

$$
\dot{y}-\dot{x} 0 \dot{x}-2 x \dot{x}=0
$$

„Da wir aber die Größe o unendlich klein angenommen haben (finxerimus), um die Momente der Größen ausdrücken zu können, können die mit ihr behafteten Terme

\footnotetext{
${ }^{10}$ [Becker 1975], S. 149; Allerdings habe ich hier ein anderes Beispiel zur Illustration gewählt.
} 
für nichts geachtet werden im Vergleich zu den anderen Termen. Ich vernachläs-

sige sie also, und es bleibt übrig:"11

$$
\dot{y}-2 x \dot{x}=0 \Leftrightarrow \dot{y}=2 x \dot{x}
$$

Die Umformung zur heute üblichen Schreibweise

$$
\dot{y}-2 x \dot{x}=0 \Leftrightarrow \dot{y}=2 x \dot{x} \Leftrightarrow \frac{\dot{y}}{\dot{x}}=2 x \quad \Leftrightarrow \quad y^{\prime}=2 x
$$

kann nur bei geeigneter Interpretation der allgemeinen Größen x und y sinnvoll sein.

An dieser Stelle soll die Betrachtung von Newtons Kalkül beendet werden. Wie oben angedeutet spielen im empirischen Teil dieser Arbeit Vorstellungen, die Schülerinnen und Schüler vom Grenzwertbegriff beim Ableiten in der Mathematik und von Momentangeschwindigkeit realer Körper eine zentrale Rolle. Daher sollen hier nun Newtons zugehörigen Vorstellungen aufgezeigt werden. Diese sind für inn essentiell bei der Rechtfertigung seines Kalküls.

\section{Newtons Vorstellungen vom Grenzwert}

Zu Beginn des vorhergehenden Zitats spricht Newton von „unendlich kleinen Teilen“:

„Die Momente der fließenden Größe (d.h. ihre unendlichen kleinen Teile, durch deren Hinzukommen in unendlich kleinen Zeitteilen die Größen selbst zugleich [jugiter] vermehrt werden) [...]."

Es sieht so aus, als würde Newton mit infinitesimalen Größen rechnen. Folgendes Zitat zeigt aber, dass Newton sich nicht wirklich infinitesimale Größen vorstellt. Vielmehr legt er auf eine Vorstellung des Grenzwerts als erstes und letztes Verhältnis Wert:

„Wenn ich also im folgenden irgendwelche Größen betrachte, die gleichsam aus konstanten Partikeln bestehen, oder wenn ich kleine Kurvenstücke als gerade betrachte, so möchte ich stets nicht etwa unteilbare, sondern verschwindende teil-

${ }^{11}$ [Becker 1975], S. 150 
bare Größen, nicht Summen und Verhältnisse bestimmter Teilchen, sondern Grenzwerte von Summen und Verhältnissen verstanden wissen, [...].“12

Newton greift wieder auf die Vorstellung von Bewegungsvorgängen zurück, wenn er versucht, das Wesen des Grenzwerts zu erläutern:

\begin{abstract}
„Es besteht der Einwand, daß es kein letztes Verhältnis verschwindender Größen gebe, weil, bevor sie verschwunden seien, das Verhältnis kein letztes sei, wenn sie aber verschwunden seien, kein Verhältnis statthabe. Aber mit demselben Argument kann man behaupten, es gebe keine letzte Geschwindigkeit eines Körpers, der an einem bestimmten Orte ankommt, wo die Bewegung endet. Denn diese Geschwindigkeit sei, bevor der Körper an diese Stelle komme, nicht die letzte, wenn er sie aber erreicht habe, gebe es keine Geschwindigkeit mehr. Und die Antwort ist leicht: Unter der letzten Geschwindigkeit ist diejenige zu verstehen, mit der der Körper sich bewegt weder bevor er seine letzte Stellung erreicht hat und die Bewegung endet noch danach, sondern dann, wenn er sie erreicht, d.h. gerade diejenige Geschwindigkeit, mit der er an dieser letzten Stelle ankommt und mit der die Bewegung endet. Ebenso ist unter dem letzten Verhältnis verschwindender Größen das Verhältnis dieser Größen zu verstehen nicht bevor sie verschwinden oder nachdem sie verschwunden sind, sondern mit dem sie verschwinden..."13
\end{abstract}

Die Beschreibung von „Bewegungen, die enden und beginnen“ können die Assoziation eines Körpers nahe legen, der ausrollt und stehen bleibt. Es ist problematisch, den Zeitpunkt, "mit dem der Körper stehen bleibt“ zu nennen, da man ein (halb-) offenes Zeitintervall betrachtet. Die Geschwindigkeit des Körpers nimmt streng monoton ab, das Intervall, in dem die Geschwindigkeitswerte des Körpers liegen, ist halboffen [a ; 0). Die Geschwindigkeitswerte fallen jedoch unter jede Schranke, ihr Grenzwert ist Null. Man muss gedanklich die Zeit weiterlaufen lassen, also den Körper noch einige Zeit in Ruhe betrachten, um insbesondere das Intervall der Geschwindigkeitswerte abschließen zu können. Dies ist ein eher trivialer Fall. Wahrscheinlicher ist, dass sich Newton z.B. eine Kugel vorstellt, die auf eine Wand trifft und nach deren Aufprallgeschwindigkeit er fragt. Die Aufprallgeschwindigkeit entspricht der letzten Geschwindigkeit, da (idealisiert gesehen) die Bewegung der Kugel an der Wand endet. In Kapitel 3.2 „Messbarkeit von Momentangeschwindigkeit“ wird der „Röhrentachometer" vorgestellt, der so eine Aufprallgeschwindigkeit messen könnte.

\footnotetext{
12 [Becker 1975], S. 151

13 [Becker 1975], S. 151
} 
Dass es allgemein ein letztes Verhältnis gibt, erklärt Newton also damit, dass es eine letzte Geschwindigkeit gibt. Hier ist zunächst die Momentangeschwindigkeit eines bewegten Körpers gemeint. Es wird deutlich, dass Newton hofft, durch den Rückgriff auf seine Altagserfahrungen von Geschwindigkeiten und Bewegungsvorgängen die Sinnhaftigkeit der von ihm entwickelten Mathe matik zu untermauern. Da er hofft, so seinen Kritikern begegnen zu können, muss er wohl davon ausgehen, dass die von inm verwendeten Eigenschaften von Bewegungen auch den Alltagserfahrungen anderer Menschen entsprechen. Für der Zeit gleichartige (also „stetig fließende“) GröBen und allgemeine Wachstumsgeschwindigkeiten geht Newton ebenfalls von der Richtigkeit seiner Überlegungen bzgl. der Grenzwerten aus. Aber: Dass Schülerinnen und Schüler heute durchaus nicht zwangsläufig mit Newtons Vorstellungen von Bewegungen konform gehen, kann den Transkripten entnommen werden.

In dieser Untersuchung wurde Schülerinnen und Schülern eine Radtour-Situation vorgelegt und gefragt, ob der Radfahrer so etwas wie eine Momentangeschwindigkeit haben kann. Newton hätte dem zugestimmt und argumentiert: Die Momentangeschwindigkeit des Radfahrers ist seine Aufprallgeschwindigkeit auf eine Mauer. Die Probandinnen und Probanden ersparen dem Radfahrer dieses Schicksal. Dennoch entwickeln sie z.T. ganz ähnliche Überlegungen: Gedanklich stellen sie sich am Radweg auf und denken über die Geschwindigkeit nach, mit der der Radfahrer vorbeifährt. ${ }^{14}$ Der Ort an dem die Beobachter stehen entspricht der Mauer, auf die die Kugel prallt. Gelegentlich sagen sie aber auch: Zu einem Zeitpunkt legt man keinen Weg zurück, daher gibt es keine Momentangeschwindigkeit. ${ }^{15}$ Diese Argumentation würde Newton vielleicht so formulieren: Wenn es keine letzten Größen gibt, gibt es auch kein letztes Verhältnis. Dies entspricht aber nicht Newtons Vorstellung von Bewegungsvorgängen. Er würde entgegnen, dass es sehr wohl ein letztes Verhältnis gibt, obwohl die beiden beteiligten Größen jeweils den Grenzwert Null haben:

„Diese letzten Verhältnisse, mit denen Größen verschwinden, sind nicht wirklich Verhältnisse letzter Größen, sondern Grenzwerte, denen sich die Verhältnisse von unbegrenzt abnehmenden Größen fortgesetzt nähern und denen sie näher kom-

\footnotetext{
${ }^{14} \mathrm{vgl.z.B.}[11]$

${ }^{15} \mathrm{vgl.}$ z.B. [13]
} 
men können als irgend eine vorgegebene Differenz, bevor die Größen unbegrenzt abgenommen haben.“ ${ }^{16}$

Dass es wiederum die letzten Verhältnisse gibt, begründet Newton mit der letzten (Aufprall-) Geschwindigkeit. Newton warnt davor, die Grenzwerte von Zähler und Nenner getrennt zu betrachten. Durch die Trennung treten die beiden Grenzwert für sich auf und können fälschlich für letzte Größen gehalten werden. Beide Grenzwerte jedoch sind Null. Dieses führt zum 0/0-Problem, mit dem sich auch die Schülerinnen und Schüler der vorliegenden Untersuchung plagen.

Es bleibt die Frage, welchen Wert der Grenzwert für Newton annimmt. Das macht er in dem folgenden Lemma I deutlich:

„Lemma I. Größen, wie auch Größenverhältnisse, die zu jeder endlichen Zeit beständig der Gleichheit zustreben und vor dem Ende jener Zeit einander näher kommen als jede vorgegebene Differenz, werden schließlich gleich. ${ }^{117}$

Sein Lemma beweist Newton mit dem naheliegenden Widerspruchsbeweis. Aufgrund dieser Äußerung nennt Volkert Newton den „Urgroßvater der Grenzwertauffassung, die im 19. Jahrhundert zum Fundament der Analysis gemacht werden sollte. " ${ }^{18}$ An Newtons Lemma I kann man eine Erweiterung des Gleichheitsbegriffs erkennen.

Für Newton als Naturwissenschaftler des 17. Jahrhunderts spielten stetige Vorgänge eine wesentliche Rolle. Daher geht er prinzipiell von stetigen bzw. differenzierbaren Kurven und Funktionen aus, wie besonders an der Vorstellung von Größen, die durch stetig bewegten Indivisibilien erzeugt werden, deutlich wird: „Ich betrachte hier die mathematischen Größen nicht als aus äußerst kleinen Teilen bestehend, sondern als aus durch stetige Bewegung beschrieben.“, siehe oben „Indivisibilien und Hauptproblem“. Durch die Weiterentwicklung des Funktionsbegriffs war es später möglich, auch unstetige und diskrete Funktionen, wie z.B. Folgen zu betrachten. Die nun notwendige mathe matische Präzisierung des Stetigkeits- und Grenzwertbegriffs (ins besondere für Funktionen) wurden u.a. von Cauchy und Weierstraß geleistet.

\footnotetext{
${ }^{16}$ [Becker 1975], S. 152

17 [Becker 1975], S. 150

${ }^{18}$ [Volkert 1988], S. 91
} 
Neben der Präzisierung von Begriffen spielen aber auch geeignete Schreibweisen eine wichtige Rolle. Ein großes Problem Newtons ist sicherlich seine Schreibweise mit dem kleinen,$\varrho^{\circ,}$ da man einer Zeile der Berechnungen für sich nicht ansehen kann, ob der Grenzprozess bereits durchgeführt wurde oder nicht; ob mit heutiger Schreibweise die linke oder rechte Seite folgender Gleichung gemeint ist: $\lim _{x \rightarrow x_{0}} f(x)=f\left(x_{0}\right)$. Möglicherweise hat Newtons Zeitgenosse Berkley diese Doppeldeutigkeit zum Anlass genommen, dessen Kalkül ironisierend zu kritisieren:

\begin{abstract}
„Bisher habe ich vorausgesetzt, daß $\times$ fließt, daß $\times$ einen wirklichen Zuwachs hat, daß o etwas ist. Und ich bin durchweg von dieser Voraussetzung ausgegangen, ohne die ich nicht imstande gewesen wäre, einen einzigen Schritt zu tun. Von dieser Voraussetzung aus komme ich zu dem Zuwachs von $x^{n}$, so daß ich inn mit dem Zuwachs von $x$ vergleichen und so das Verhältnis der beiden Zuwüchse finden kann. Ich bitte dann um die Erlaubnis, eine neue Voraussetzung machen zu dürfen, die der ersten entgegengesetzt ist; $d$.h. ich will jetzt voraussetzen, daß es keinen Zuwachs von $x$ gibt oder daß o nichts ist, welche zweite Voraussetzung meine erste zerstört und mit ihr unverträglich ist und deshalb mit allem, was sie voraussetzt. Ich bitte nichtsdestoweniger um die Erlaubnis $n x^{n-1}$ zurückzubehalten, welches ein Ausdruck ist, der vermöge meiner ersten Voraussetzung erhalten wurde, ja welcher notwendig eine solche Voraussetzung voraussetzt und nicht ohne sie erhalten werden könnte.

All das scheint mir eine sehr widerspruchsvolle Art von Argumentation zu sein, so wie sie in der Theologie nicht erlaubt wäre." ${ }^{19}$
\end{abstract}

\title{
Newtons Vorstellungen und Schulanalysis
}

Aus heutiger Sicht hat Newton noch keinen formalen Grenzwertbegriff. Seine Vorstellungen könnte man vielleicht mit einem eher intuitiven Grenzwertbegriff, wie er für den Mathematikunterricht in den 11. Klassen und für Mathematik-Grundkurse in Nordrhein-Westfalen gefordert wird (vgl. [NRW 1999]), vergleichen. Newtons Leistung liegt nicht in seinem Kalkül, der zu seiner Zeit ja bereits bekannt und verreitet war, sondern in den zugehörigen Vorstellungen vom Grenzwertbegriff, die im Lemma I zum Ausdruck kommen. Newton gründet sie auf Vorstellungen von Bewegungsvorgängen, insbesondere auf Vorstellungen von der Momentangeschwin-

\footnotetext{
${ }^{19}$ vgl. [Becker 1975], S. $157-158$
} 
digkeit. Die Überlegungen zu Bewegungsvorgängen können mit Grundvorstellungen $^{20}$ des Ableitungsbegriffs, wie sie für den Mathe matikunterricht gefordert werden, in Verbindung gebracht werden.

Wie die Transkripte dieser Untersuchung zeigen, bereiten Schülerinnen und Schülern Überlegungen zur Momentangeschwindigkeit jedoch Probleme. Im Alltag wird der Begriff „Momentangeschwindigkeit“ bedenkenlos im Sinne Newtons verwendet, vgl. Kap. 3.1. Denn: Wenn man sich die ganze Zeit bewegt, hat man doch zu jedem Zeitpunkt eine Geschwindigkeit. Also gibt es letzte Verhältnisse. Aus den Transkripten ergibt sich auch, dass das intensive Nachdenken über Momentangeschwindigkeit Menschen dazu bringen kann, diese Alltagsbedeutung in Frage zu stellen: Zu einem Zeitpunkt legt man schließlich keinen Weg zurück, wie soll man da eine Momentangeschwindigkeit haben können? Also gibt es keine letzten Verhältnisse. - Kommt man zu dem Ergebnis, dass es keine letzten Verhältnisse gibt, schließt sich die Frage an, ob der Wert der Momentangeschwindigkeit mit Hilfe der Weg-Zeit-Funktion und der Differenzialrechnung überhaupt berechnet werden kann. Das scheint wiederum ein Widerspruch dazu zu sein, dass die Differenzialrechnung die Verbindung zwischen Weg-Zeit-Funktion und Geschwindigkeit-Zeit-Funktion zwingend herstellt, wie an Newtons Vorstellungen gezeigt wurde.

Im Kapitel 4 dieser Arbeit versuche ich, geeignete Begriffe zur genaueren Beschreibung der gezeigten Denkweisen und für Ursachen für diese zu entwickeln. Dazu wird im Folgenden zwischen Überlegungen in der realen Altagswelt ( $r$-Welt) und der Welt der Mathematik ( $m$-Welt) unterschieden ${ }^{21}$. Es ist notwendig, sich zunächst mit dem Begriff der Momentangeschwindigkeit ( $r$-Welt), dem Grenzprozess ( $r$ - und $m$-Welt) und dem Grenzwertbegriff bei Funktionen (m-Welt) auseinanderzusetzen. In Abbildung 2 ist der Zusammenhang dieser Begriffe skizziert.

\footnotetext{
${ }^{20}$ Zum Begriff der Grundvorstellung vgl. z.B. [Bender 1991a], [Blum/Törner 1983] und [vom Hofe 1995a]

${ }^{21}$ Zu den Denkwelten vgl. Kap. 2.2
} 


\section{m-Welt}

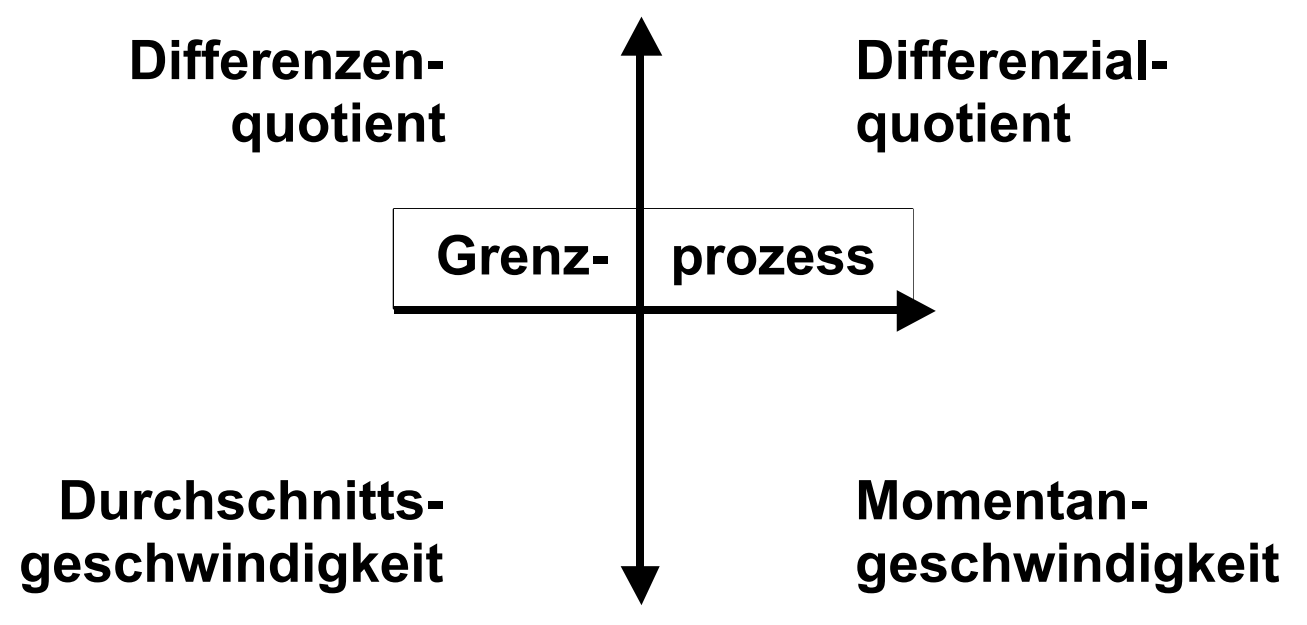

$r$-Welt

Abbildung 2: Skizze der zentralen Begrifflichkeiten in den Kapiteln 3 und 4 


\section{Zum Design der Untersuchung}

In diesem Kapitel soll zunächst die Aufgabe, die den Schülerinnen und Schülern vorgelegt worden war, vorgestellt und diskutiert werden (Kap. 2.1). In Kap. 2.2 finden sich einige Bemerkungen zum Denkwelt-Begriff. Zur Interpretation und Analyse habe ich Methoden der empirischen Unterrichtsforschung angewendet, jedoch nicht puristisch, sondern in einer, wie ich meine, fruchtbaren Verbindung mit der Sachanalyse mathematischer Begrifflichkeiten (Kap. 2.3). Für diese Art der Arbeit gibt es in jüngerer Zeit vermehrt positive Beispiele in der Gemeinschaft der deutschsprachigen Mathematikdidaktik, siehe z.B. vom Hofe und Hölzl. Darüber hinaus habe ich die in der explorativen Sozialforschung bekannte Methode der Grounded Theory herangezogen. In Kap. 2.4 folgen schließlich einige Bemerkungen zur Findung und Zusammensetzung der Gruppe der Probandinnen und Probanden zu finden.

\subsection{Die Aufgabe}

Folgende Aufgabe besteht aus fünf Teilaufgaben. Jede Teilaufgabe soll schriftlich bearbeitet werden.

a) Jan Ulrich macht eine Radtour. „Zufällig“ fährt er genau so, dass die Funktion f mit $f(t)=\frac{28 t(10-t)}{5}$ für die ersten 5 Stunden wiedergibt, wie weit er bis zu einem Zeitpunkt schon vorangekommen ist. Start ist um 14:00 Uhr.

Folgende Wertetabelle gibt einige Entfernungen an, die Jan Ulrich bis zum jeweiligen Zeitpunkt zurückgelegt hat:

\begin{tabular}{|l|l|l|l|l|l|l|l}
\hline Zeitpunkt & $14: 30$ & $15: 00$ & $15: 30$ & $16: 00$ & $17: 00$ & & \\
\hline Fahrdauer in $\mathrm{h}$ & 0.5 & 1 & 1.5 & 2 & & & \\
\hline $\begin{array}{l}\text { Zurückgelegter } \\
\text { Weg in km }\end{array}$ & 26.6 & 50.4 & 71.4 & 89.6 & & & \\
\hline
\end{tabular}

Wie weit ist er nach 3 Stunden gekommen?

b) Nach den ersten drei Stunden der Tour will der Trainer wissen, wie schnell Jan in diesen drei Stunden durchschnittlich gefahren ist. Auch interessieren inn die Durchschnittsgeschwindigkeiten für die letzten 2 1/2 Stunden, die letzten 2 Stunden, die letzten 1 1/2Stunden, die letzte Stunde und die letzte halbe Stunde.

c) Bisher war immer von Durchschnittsgeschwindigkeiten die Rede. Gibt es auch so etwas wie eine Momentangeschwindigkeit um 16:00 Uhr? Begründe! 
d) Wie kannst Du möglichst genau die Momentangeschwindigkeit berechnen?

e) Überlege, ob und wie Du mit Deiner Methode die Momentangeschwindigkeit ganz genau berechnen kannst.

\section{Diskussion der Teilaufgaben}

Jede der fünf Teilaufgaben der Aufgabe stand auf einem eigenen Blatt Papier. Die Schülerinnen und Schüler hatten daher immer genügend Raum, ihre Bearbeitungen unter der Teilaufgabe zu notieren. Außerdem sollten sie die nächste Teilaufgabe erst dann aufdecken, wenn die vorherige fertig bearbeitet worden war. Dadurch sollte verhindert werden, dass sie Aufgabenteile im Voraus lasen. Wie man an der Aufgabenstellung sieht, ist das sinnvoll, da die Aufgabenstellung einer späteren Teilaufgabe Hinweise auf die Bearbeitung der vorhergehenden Teilaufgaben geben kann. Jeweils zwei Schülerinnen und Schüler haben die Aufgabe gemeinsam, also nicht im Wettkampf bearbeitet. Diese Arbeitsphase wur de gefilmt und später transkribiert.

\section{Zu Aufgabenteil a)}

Dieser Aufgabenteil dient hauptsächlich der Einarbeitung in die modellierte Situation. Insbesondere macht er darauf aufmerksam, dass es zwei Typen von Zeitangaben gibt: die Uhrzeit und die Fahrdauer, wobei die Uhrzeit einen Zeitpunkt, und die Fahrdauer eben eine Zeitdauer wiedergibt ${ }^{22}$. Die Fahrdauer liefert Werte für das Argut ment der Funktion. Durch die Aufgabe werden also erste Zusammenhänge zwischen $\mathrm{r}$ - und $\mathrm{m}$-Welt vorgegeben.

Die Aufgabenstellung, der Auftrag, einen Funktionswert zu berechnen, ist schulüblich und wurde fast immer schnell erfüllt. Doch manchmal ist den Schülerinnen und Schülern nicht klar, wie sie den gesuchten Wert ermitteln sollen. Dann treten verschiedene Strategien auf: Den Videoaufnahmen ist zu entnehmen, dass viele Schülerinnen und Schüler, die zumindest eine Ahnung haben, wie sie vorgehen können, zunächst Fahrdauern in $f$ einsetzen, zu denen die zugehörigen Entfernungen bereits in der Wertetabelle vorgegeben waren. So testen sie ihr geplantes Vorgehen ${ }^{23}$. An-

\footnotetext{
$\overline{22}$ Die daraus entstehende Problematik wird in Kap. 3.2 „Weg-Zeit- oder Orts-Zeit-Funktion?“ ausführlich besprochen.

${ }^{23} \mathrm{vgl.} \mathrm{z.B.} \mathrm{[01],} \mathrm{[02],} \mathrm{[05],} \mathrm{[10],} \mathrm{[12]}$
} 
dere versuchen, die gesuchte Strecke mit Hilfe der gegebenen Strecken zu berechnen. Dabei lässt sich mehrfach die Idee finden, dass die Orts-Zeit-Funktion linear sein könnte / müsste ${ }^{24}$. Wieder anderen ist die Bedeutung der Funktion nicht klar. Wenn sie die Variable t mit dem Wort „time“ in Verbindung bringen, klärt sich die Aufgabenstellung ${ }^{25}$. - Die leeren Spalten der Wertetabelle haben offensichtlich einen hohen Aufforderungscharakter: Gelegentlich beginnen Probandenpaare damit, die Tabelle vollständig auszufüllen ${ }^{26}$.

Ein Grund für (aus Lehrersicht unverständlich) komplizierte Arbeitsansätze könnte sein, dass die Schülerinnen und Schüler versteckte Fallen und Tricks erwarten, da innen bewusst ist, dass sie Probandinnen und Probanden eines Forschungsversuchs sind: „Das ist doch ne Falle von inm.“ 27

\section{Zu Aufgabenteil b)}

Die Einführung der nur indirekt gegebenen Durchschnittsgeschwindigkeit (neben den direkt gegebenen und schon bekannten Entfernungen) erweitert und vertieft die Modellierung der Situation und stellt eine weitere Verbindung zwischen den Denkwelten her. Bis hier waren die Schülerinnen und Schüler hauptsächlich gefordert, die durch die Aufgabenstellung vorgegebene Modellierung nachzuvollziehen. Nun soll ein erster eigener Schritt, selber eine Entsprechung zwischen $r$ - und m-Welt zu entwickeln, geleistet werden, da die Berechnungsvorschrift für die Durchschnittsgeschwindigkeit nicht gegeben ist. Insbesondere die Erkenntnis, dass Durchschnittsgeschwindigkeiten der „letzten x Stunden“ berechnet werden sollen, also Wegdiffe renzen betrachtet werden müssen, führt dazu, dass die Probanden selber ein wenig Modellbildung betreiben müssen.

Um die letzte der geforderten Durchschnittsgeschwindigkeiten berechnen zu können, müssen die Probanden einen weiteren Funktionswert berechnen. Hier kann man nun einen Zweck der beiden ersten Aufgabenteile erkennen: Später werden GrenzwertBetrachtungen am Differenzenquotienten gefordert. In Aufgabenteil b) wird der Differenzenquotient teilweise aufgestellt. Teilweise, da hier im Nenner die Zeitdifferenz

\footnotetext{
${ }^{24}$ vgl. z.B. [02], [12]

${ }^{25}$ vgl. z.B. [02], [12]

${ }^{26}$ vgl. z.B. [11]

${ }^{27}[11,127]$
} 
nicht betrachtet werden muss, da der Wert der Differenz im Aufgabentext angegeben wird. Um Grenzwert-Betrachtungen anstellen zu können, muss unter anderem sichergestellt sein, dass man in einer Umgebung um die betrachtete Stelle alle Funktionswerte der gegebenen Funktion $f$ berechnen kann. Dass möglicherweise Funktionswerte berechnet werden müssen, wird durch den Aufgabenteil b) betont. Dass Funktionswerte berechnet werden können, zeigt Aufgabenteil a).

Die Berechnung der Durchschnittsgeschwindigkeitswerte und der Schwierigkeitsgrad der Modellbildung bewegen sich im Rahmen schulüblicher Aufgaben. Die Videoaufnahmen zeigen, dass viele Schülerinnen und Schüler die Aufgabenstellung zunächst nicht aufmerksam lesen: Oft werden zunächst die Durchschnittsgeschwindigkeiten der ersten $\times$ Stunden berechnet ${ }^{28}$. Der Begriff der Durchschnittsgeschwindigkeit ist nicht allen Schülerinnen und Schüler in seiner r-Welt-Bedeutung sofort geläufig. Daher wird der Differenzenquotient als zugehöriges mathematische Objekt nicht immer aufgestellt: Stattdessen werden die zurückgelegten Kilometer der Spalten der Wertetabelle aufaddiert, um sie durch die Gesamtzahl der Werte zu dividieren ${ }^{29}$. Mit dem Begriff „Durchschnitt“ wird hier wohl das arithmetische Mittel assoziiert, also mit dem m-Welt-Algorithmus „addieren und durch Anzahl der Summanden teilen“ verbunden. Gelegentlich soll die Summe auch durch die gesamte Fahrdauer geteilt werden.

Die Schülerinnen und Schüler wenden verschiedene Techniken an, um zu überprüfen, ob die von innen berechneten Werte der Durchschnittsgeschwindigkeiten richtig sein können. Unter Einbezug der r-Welt wird z.B. gefragt: „Ist so eine Durchschnittsgeschwindigkeit für einen Radfahrer realistisch?“ Oder: „Warum wird der immer langsamer?" Aber auch in der m-Welt werden Vergleiche mit vorangegangenen Rechenergebnissen angestellt ${ }^{30}$.

Bis hier werden weder in der $r$-noch in der m-Welt infinitesimale Denk- und Rechenweisen benötigt. Der realen Situation entsprechen konkrete Entfernungen und Durchschnittsgeschwindigkeiten, also Werte, die sich mit Hilfe von Kalkülen aus der Sekundarstufe I berechnen lassen. Dies ändert sich nun in den Aufgabenteilen c) und d),

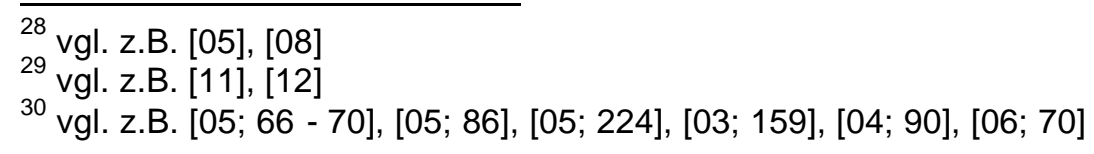


in denen die Schülerinnen und Schüler einen ersten Zusammenhang zwischen Durchschnittsgeschwindigkeit und Momentangeschwindigkeit in der r-Welt einerseits und dem Grenzprozess am Differenzenquotient in der m-Welt andererseits herstellen sollen. In Aufgabenteil e) schließlich geht es, mathe matisch betrachtet, um die Momentangeschwindigkeit und den Differenzialquotienten.

\section{Zu den Aufgabenteilen c) und d)}

In Aufgabenteil c) wird gefragt, ob es auch so etwas wie eine Momentangeschwindigkeit gibt. Es bleibt offen, in welcher Denkwelt diese Frage beantwortet werden soll. In der r-Welt ist diese Frage verwirrend. Jeder, der schon einmal Fahrrad, Auto oder Zug gefahren ist, weiß, dass es eine Momentangeschwindigkeit gibt. Geschwindigkeit ist eine Altagserfahrung, die jede Schülerin und jeder Schüler gemacht hat (vgl. 3.1). Die Momentangeschwindigkeit kann sogar mit einem Altagsinstrument, einem Tachometer, gemessen werden (vgl. 3.2). Die Erfahrung "Geschwindigkeit" und die Messbarkeit der Momentangeschwindigkeit lassen also die Frage nach der Existenz derselben verwirrend und überflüssig erscheinen. Dies empfinden wohl auch die Probandinnen und Probanden, wie man an verschiedenen Stellen der Transkripte erkennen kann. Sie stehen vor dem Problem, etwas zu begründen, das so alltäglich ist, dass es eigentlich keiner Begründung bedarf. Die Altagserfahrung und die Messbarkeit sind aber Elemente der r-Welt.

Die Transkripte zeigen, dass das Problem, etwas in der $\mathrm{r}$-Welt Selbstverständ liches begründen zu müssen, die Probanden dazu bringt, die Begründung außerhalb der rWelt zu suchen. Als der Versuchsleiter die Schülerinnen und Schüler für seinen Versuch geworben hat, hat er sich als angehender Mathe matiklehrer vorgestellt und die Untersuchung in den Kontext "Schülerinnen, Schüler und Mathematik" eingebettet. Da den Schülerinnen und Schülern der mathe matische Charakter der Untersuchung und der Aufgabe bewusst ist, und innen die Fragestellung in der r-Welt überflüssig erscheint, fühlen sie sich wohl angehalten, die Begründung in der m-Welt zu suchen. Schülerinnen und Schüler stellen für sich Annahmen auf, was sie meinen, was von innen erwartet wird. Diese Annahmen unterstützen wohl diesen Wechsel in die $\mathrm{m}$ Welt. - Die Aufgabe heißt „Begründe!“. Natürlich sind auch in der m-Welt Begründungen möglich. Allerdings werden oft zunächst Berechnungsversuche als Bearbeitung dieses Aufgabenteils angeboten. Die Schülerinnen und Schüler interpretieren die 
Aufgabenstellung um: von „Gibt es eine Momentangeschwindigkeit?" in „Wie können wir die Momentangeschwindigkeit berechnen?" Dieser Wechsel zeigt, welches Bild von Mathematik die Schule bei den Schülerinnen und Schülern erzeugen kann: Mathematik kann eng mit „Rechnen“ verbunden, oder sogar gleichgesetzt werden. Zudem kann die Erwartungshaltung, die dem Versuchsleiter von den Schülerinnen und Schülern möglicherweise zugeschrieben wird, eine Rolle spielen: Sie meinen vielleicht, dass in einer mathematischen Untersuchung Rechenfertigkeiten gefordert seien. Für die Frage nach der Existenz der Momentangeschwindigkeit mag der Gedanke dahinterstecken, dass die Berechnung des Wertes die Existenz der Momentangeschwindigkeit sicherstellt. Schülerinnen und Schüler, die von dieser Annahme ausgehen, können diesen Aufgabenteil natürlich durch die Berechnung des Werts der Momentangeschwindigkeit beantworten.

Eine alternative Formulierung dieses Aufgabenteils könnte etwa so aussehen:

„Bisher war von Durchschnittsgeschwindigkeiten die Rede.

i) Begründe, warum es auch eine Momentangeschwindigkeit gibt.

ii) Berechne diese möglichst genau."

Allerdings halte ich diese Formulierung für die vorliegende Untersuchung für nicht geeignet, da die zweite Frage die m-Welt, und somit die erste (als Alternative gesehen) die r-Welt betont. Diese alternative Aufgabenstellung würde vermutlich eine Trennung der beiden Welten hervorheben, so dass die Bemühungen der Schülerinnen und Schüler, die Welten in Einklang zu bringen, möglicherweise gemindert oder sogar verhindert worden wäre, so nach der Idee: Die Antworten zu zwei getrennten Fragen müssen nichts miteinander zu tun haben. Diese Haltung wäre der Untersuchung abträglich gewesen.

Die Transkripte zeigen, dass die Probandinnen und Probanden bei der Bearbeitung dieser Teilaufgabe in einem ständigen Spannungsverhältnis der beiden Denkwelten stehen $^{31}$. Dieses Spannungsverhältnis wird vom Versuchsleiter als produktiv und im positiven Sinne als provokativ empfunden. Den Transkripten der Vor- und Hauptuntersuchung ist zu entnehmen, dass den Probandinnen und Probanden intuitiv sehr

${ }^{31}$ vgl. [06] und dessen Interpretation in Kap. 5.1 
wohl der Unterschied zwischen den beiden Denkwelten klar ist, und es gibt auch Gegenüberstellungen der Welten. Aber vor allem gibt es die Bemühungen, die Welten in Einklang zu bringen.

Die Untersuchung zeigt auch, dass die Frage der Teilaufgabe c) gelegentlich zunächst nicht beantwortet werden kann. Manchmal wird ein Berechnungsversuch auch dann angeboten, wenn den Probandinnen und Probanden intuitiv klar ist, dass die Aufgabe nicht explizit eine Berechnung fordert.

Der Aufgabenteil d) fordert nun eine möglichst genaue Berechnung der Momentangeschwindigkeit, betont also die m-Welt. Hier stutzen viele Schülerinnen und Schüler, da sie hier eine Aufgabenstellung finden, die sie zu dem auffordert, was sie als Bearbeitung der vorhergehenden Teilaufgabe bereits getan haben. Durch die Bearbeitung des Aufgabenteils c) aber ist für alle Schülerinnen und Schüler klar, dass es einen Zusammenhang zwischen $r$ - und $m$-Welt geben muss. Daher kommt es nicht zu den oben befürchteten Auswirkungen, zu separaten Betrachtungen in beiden Welten. Allerdings wird wohl die Existenz der beiden Denkwelten so ins Bewusstsein der Schülerinnen und Schüler gehoben, dass oft eine erneute Bearbeitung des Aufgabenteils $\mathrm{c}$ ) angeregt wird. Das hat einerseits den Vorteil, dass nun zu beiden Aufgabenteilen Antworten aufgeschrieben werden können. Andererseits gibt die Neubearbeitung den Mut, als Antwort von Aufgabenteil c) eine Begründung durch intensive Beschreibung der Altagserfahrung "Geschwindigkeit" zu geben, wobei dazu oft mathe matische Vokabeln gebraucht werden.

Die typischen Denkweisen, die die Schülerinnen und Schüler hier an den Tag legen, sollen ausführlich in Kapitel 4 beschrieben werden. Es zeigte sich jedoch, dass die Schwierigkeiten dieser Aufgabenteile darin bestehen, einerseits den FWelt-Begriff „Momentangeschwindigkeit" zu klären, andererseits in der m-Welt den Grenzprozess am Differenzenquotienten zu initiieren, dann dessen Bedeutung für die $\mathrm{FWelt} \mathrm{zu}$ klären, und schließlich die jeweils zugehörigen Begrifflichkeiten beider Denkwelten zu verbinden.

Solche Begründungsaufgaben tauchen eher selten in Schulbüchern der Sekundarstufe I und II auf. Daher kann die Bearbeitung nicht auf Routine-Tätigkeiten zurück- 
geführt werden. Ein Paar ${ }^{32}$, eine Schülerin und ein Schüler, die beide sehr gute Noten im Mathematik-Leistungskurs erzielten, berechnete im Aufgabenteil c) bereits den Wert der Ableitung. Mit den beiden letzten Aufgabenteilen konnten die beiden nichts anfangen. Daher beklagten sich im abschließenden Gespräch mit dem Versuchsleiter darüber, dass dreimal die selbe Aufgabe gestellt wurde. Zwar gelang es diesem, den unterschiedlichen Charakter der Aufgabenteile zu verdeutlichen, aber anschließend beurteilten die Schülerin und der Schüler die Teile c) und d) so: Das hat nichts mit Mathematik zu tun. - Eine Dreier-Kandidatin ${ }^{33}$ eines Grundkurses antwortete auf die Klagen ihrer Mitstreiterin über die blöde Begründungsaufgabe: „Das ist doch gut, das machen wir in der Schule nie." Aus der Bemerkung der DreierKandidatin kann man den Schluss ziehen, dass einige Lehrerinnen und Lehrer vielleicht zu starke Bedenken gegenüber Begründungsaufgaben haben, die im Vergleich zu Berechnungsaufgaben als deutlich schwerer angesehen werden.

\section{Aufgabenteil e)}

Der Aufgabenteil e) regt an, noch einmal über das Verhältnis von $r$ - und $m$-Welt entlang eines Bogens zwischen Momentangeschwindigkeit und Differenzenquotient nachzudenken. Leider wird dieser Aufgabenteil der inm zugedachten Funktion nicht immer gerecht. Oft kommt es nur zu einer Wiederholung von Argumenten, die bereits in den vorhergehenden Aufgabenteilen genannt wurden. Gelegentlich geben die Probanden nach der Bearbeitung von Aufgabenteil d) auf. Oft haben die Schülerinnen und Schüler schon 40 Minuten konzentriert gearbeitet, bis sie zu dieser Teilaufgabe gelangen. Daher haben sich bei e) wahrscheinlich Ermüdungserscheinungen und ein Nachlassen der Motivation bemerkbar gemacht.

Manchmal jedoch regt der Aufgabenteil e) die Schülerinnen und Schüler dazu an, zuvor begonnene Gedanken wieder aufzunehmen und weiter zu treiben. Weil nun alle Aufgabenteile bekannt sind, können die Schülerinnen und Schüler die Intentionen, die sie dem Versuchsleiter bzgl. der einzelnen Aufgabenteilen zuschreiben, neu interpretieren. Das führt vielleicht dazu, dass Begründungen in den Bearbeitungen zu c) und d) noch einmal verbessert werden, da ja nun klar ist, dass am ehesten im letzten Aufgabenteil gerechnet werden soll.

\footnotetext{
${ }^{32}$ vgl. [16]

${ }^{33} \mathrm{vgl}$. [06]
} 


\section{Bezüge zur Schulpraxis}

In dieser Arbeit zeigt sich das persönliche Interesse des Autors an den Fragen nach den Vorstellungen vom Grenzwertbegriff bei Schülerinnen und Schüler. Folgen und Reihen mit ihren Grenzwerten werden inzwischen eher selten an Gymnasien unterrichtet ${ }^{34}$. Aus diesem Grund wurde die oben aufgeführte Aufgabe aus dem Bereich der Differenzialrechnung gewählt, bei der der Grenzwertbegriff immer noch eine zentrale Rolle spielt.

In der didaktischen Literatur werden oft drei Grundvorstellungen für den Grenzprozess der Differenzialrechnung vorgestellt: die Sekanten-Tangenten-Vorstellung, die Vorstellung von der durchschnittlichen und lokalen Änderungsrate und die Vorstellung der linearen Approximation ${ }^{35}$. In Kap. 4.3 wird gezeigt, dass es letztlich nur zwei verschiedene Grundvorstellungen gibt, da die Vorstellung der Tangentensteigung ein Spezialfall der Vorstellung der lokale Änderungsrate ist. Der bis August 1999 gültige Lehrplan für Mathematik für die gymnasiale Oberstufe des Landes NordrheinWestfalen ([NRW 1981]) betont als Zugang zur Differenzialrechnung ausschließlich die Tangentensteigungsvorstellung der Ableitung ${ }^{36}$. Der Zugang über diese Vorstellung wird oft als geometrisch und innermathe matisch bezeichnet ${ }^{37}$, da er zur Begründung des Differenzenquotienten und des Grenzprozesses aus schließlich mit mathematischen Objekten auskommt: Graphen von Funktionen, Sekanten, Tangenten und Steigungsdreieck, Differenzen- und Differenzialquotient und lim-Operator. Dem gegenüber steht der sogenannte außermathe matische Zugang, der mit der Vorstellung der lokalen Änderungsrate in Verbindung gebracht wird. „Außermathematisch“ ist hier im Vergleich zum innermathematischen Zugang nur die zusätzliche Einkleidung in einen außermathematischen Kontext. Als Anwendung der Differenzialrechnung findet man im genannten Lehrplan ${ }^{38}$ für Grundkurse im Unterthema „3. Anwendungen“" nur die Kurvendiskussion, die Bestimmung von Funktionsvorschriften von Funktionen mit gegebenen Eigenschaften und die Charakterisierung einfacher Funktionenklassen. Das entsprechende Unterthema für Leistungskurse sieht zudem eine

\footnotetext{
${ }^{34}$ Das kann der Autor aus eigener Erfahrung sagen, die z.B. von Bender geteilt wird, vgl. [Bender 1991b]. Seit kurzem unterstützt auch der Lehrplan des Landes Nordrhein-Westfalens die Vernachlässigung von Folgen und Reihen. Für Leistungskurse wird vorgeschlagen, Folgen und Reihen im Anschluss an die Differenzialrechnung zu betrachten, vgl. [NRW 1999].

${ }^{35} \mathrm{vgl.}$. z.B. [Blum/Törner 1983]

${ }^{36}$ [NRW 1981], S. 36, 38

${ }^{37} \mathrm{vgl}$. [Blum/Törner 1983] Kap. A5

${ }^{38}$ [NRW 1981], S. 37
} 
„exemplarische Anwendung in einer außermathe matischen Wissenschaft" vor $^{39}$. Hier kann man Ansätze jener antididaktischen Umkehrung erkennen, die Freudenthal beschreibt, vgl. Kap 1.1.

Im neuen, seit August 1999 gültigen Lehrplan für die gymnasiale Oberstufe [NRW 1999] hat aber nun ein Tausch stattgefunden. Als primäre Grundvorstellung wird nun die lokale Änderungsrate vorgeschlagen, die mit Hilfe der Tangentensteigung visualisiert werden kann. Dies ist ein Grund dafür, dass in der Aufgabe dieser Untersuchung die lokale Änderungsrate an einem Bewegungsproblem thematisiert wird. Die Schülerinnen und Schüler wurden zwar noch nach dem alten Lehrplan unterrichtet, jedoch gab es zwei Paare aus zwei Kursen (derselben Lehrerin), die den Einstieg in die Differenzialrechung über ein Bewegungsproblem kennengelernt hatten.

\subsection{Bemerkung zum Begriff der Denkwelt}

\section{Denkwelten und Modellbildung}

Mehrfach war von Denkwelten die Rede. Im Folgenden wird geklärt, wie dieser Begriff hier verstanden wird. Ähnlich wie in der Modellbildungstheorie sollen die reale Alltagswelt und die Mathematik-Welt gedanklich einerseits gegenübergestellt und andererseits miteinander verbunden werden. In einer Denkwelt denkt man über einen bestimmten Gegenstandsbereich nach. Die beiden Gegenstandsbereiche dieser Arbeit sind Bewegungsvorgänge in der realen Alltagswelt und die Differenzialrechnung in der Mathe matik. Die Denkwelten zu diesen Gegenstandsbereichen sollen $\mathrm{r}$ - und m-Welt genannt werden. Sie „enthalten" Vorstellungen und gedachte Strukturen des jeweiligen Gegenstandsbereichs und können mit Modellen der Modellbildungstheorie verglichen werden. Ähnlich, wie hier ein Gegenstandsbereich und die zugehörige Denkwelt gegenübergestellt werden, ist in der Modellbildungstheorie oft von dem „realen Problem“ und dem „realen Modell“, sowie von dem „mathematischen Modell“

${ }^{39}$ [NRW 1981], S. 39 
und der „mathematischen Lösung“ u.ä. die Rede ${ }^{40}$. Modelle im Sinne der Modellbildungstheorie charakterisiert Stachowiak so:

\section{„1. Abbildungsmerkmal:}

Modelle sind stets Modelle von etwas, nämlich Abbildungen und damit Repräsentationen gewisser natürlicher und künstlicher Originale, die selbst wieder Modelle sein können." ${ }^{41}$

Stachowiaks „natürliche und künstliche Originale“ entsprechen den, in der r-Welt eher natürlichen, in der m-Welt eher künstlichen Situationen. Modelle zu diesen Originalen finden sich in den Denkwelten. Der m-Welt können sowohl künstliche Originale als auch Modelle zugeschrieben werden. Das r-Welt-Modell „Weg-ZeitFunktion“ einer realen Situation „Bewegungsvorgang“ kann Original in der mWelt sein. Hier deutet sich an, dass es sehr schwer sein wird, Äußerungen der Schülerinnen und Schüler genau einer Denkwelt zuzuschreiben. Mehr als eine Betonung einer Denk welt wird man selten feststellen können.

\section{„2. Verkürzungsmerkmal:}

Modelle erfassen nicht alle Eigenschaften des durch sie repräsentierten Original(system)s, sondern nur solche, die den jeweiligen Modellerschaffern und -benutzern relevant erscheinen." 42

Verkürzungen lassen sich in den Transkripten finden. Über triviale wie die Unerheblichkeit der Farbe des Fahrrads soll nichts weiter gesagt werden. - Im Alltag und somit auch für die Schülerinnen und Schüler wird unter dem Begriff „Geschwindigkeit“ der physikalische Begriff des „Tempos“ verstanden. Zum physikalischen Geschwindigkeitsbegriff gehört neben dem Tempo auch die Richtung, die für die Schülerinnen und Schüler keine Rolle spielt. Oft stellen sie sich geradlinige Bewegungen vor, wie Handbewegungen einiger Schülerinnen und Schüler auf den Videofilmen ${ }^{43}$ vermuten lassen. Andere Schülerinnen und Schüler stellen sich gedanklich an dem Ort auf, an dem der Radfahrer um 16 Uhr vorbei kommt, „wie Jan Ullrich nun angeradelt kommt“ ${ }^{44}$. Hier wiederum kann man unterstellen, dass dieser sich nicht

\footnotetext{
${ }^{40}$ vgl. z.B. [Blum 1985]

41 [Stachowiak 1973], S. $131-133$

42 [Stachowiak 1973], S. $131-133$

43 z.B. [06, 190], $[14,170]$

${ }^{44}[11,291]$
} 
geradlinig bewegt. Das wird in den Transkripten aber nicht thematisiert. In der Physik kann die Geschwindigkeit durch einen Vektor repräsentiert werden, dessen Fußpunkt sich an dem Punkt eines Koordinatensystem befindet, der den Ort des Radfahrers zu einem Zeitpunkt darstellt. Die Richtung des Vektors entspricht der momentanen Richtung des Radfahrers, seine (absolute) Länge dem Tempo. Im Alltagsverständnis wird die Geschwindigkeit durch eine Zahl repräsentiert. Indem die Schülerinnen und Schüler den Geschwindigkeitsbegriff in seiner Alltagsbedeutung benutzen, verkürzen sie inn aus Sicht des Physikers. - Der Radfahrer bewegt sich auf einer Kurve durch den dreidimensionalen Raum. Für Schülerinnen und Schüler ist die Länge eines Kurvenabschnitts oder ein Ort, an dem der Radfahrer vorbei fährt, intuitiv existent. Der Mathe matiker müsste, um die Länge eines Kurvenabschnitts im $\mathrm{R}^{3} \mathrm{zu}$ bestimmen, rektifizieren, und um einen Ort im Raum zu beschreiben, einen Vektor und ein Koordinatensystem bemühen. Eine Verkürzung findet hier also nur aus der Sicht des Mathematikers statt, nicht aus der Alltagssicht.

\section{„3. Pragmatisches Merkmal:}

Modelle sind ihren Originalen nicht per se eindeutig zugeordnet. Sie erfüllen ihre Repräsentations - und Erzeugungsfunktion vielmehr immer nur
a) für bestimmte Subjekte
b) unter Einschränkungen auf bestimmte gedankliche oder tatsächliche Opera- tionen und
c) innerhalb bestimmter Zeitspannen.“ ${ }^{45}$

Zu Aspekt a): Tatsächlich zeigt sich in den Transkripten, dass verschiedene Schülerinnen und Schüler verschiedene Vorstellungen z.B. von Bewegungsvorgängen entwickeln: Sie stellen sich am Straßenrand auf, betrachten den Radfahrer über eine gewisse Zeitdauer der Radtour oder nur zu dem Zeitpunkt $16 \mathrm{Uhr}^{46}$. Andere stellen sich vor, sie säßen in einem Auto und beobachteten den Tachometer ${ }^{47}$. Wieder andere verdeutlichen sich Bewegungsvorgänge anhand von Handbewegungen ${ }^{48}$. Von diesen Vorstellungen hängt ab, wie sie den Aufgabenteil c) beantworten.

\footnotetext{
${ }_{46}^{45}$ [Stachowiak 1973], S. $131-133$

46 vgl. [06; 210], [11, 291], [14; 171], [13; 245]

47 z.B. $[06 ; 210-216]$

${ }^{48}$ z.B. $[14 ; 168-172]$
} 
Zu Aspekt b): Aus der Weg-Zeit-Funktion kann durch eine Operation leicht die Geschwindigkeit-Zeit-Funktion gewonnen werden. Dafür ist die Weg-Zeit-Funktion als Modell eines Bewegungsvorgangs gut geeignet. Völlig ungeeignet ist sie dagegen, wenn man z.B. herausfinden will, ob die Radtour in einem gewissen Abschnitt besonders anstrengend war oder nicht: Wird dort eine große Weglänge zurückgelegt, kann sich der Radfahrer besonders angestrengt haben, oder aber er ließ sich entspannt einen steilen Berg herabrollen.

Zu Aspekt c): In Kapitel 4 werden zwei Sichtweisen vorgestellt, die die Schülerinnen und Schüler unabhängig von den Denkwelten entwickeln. Diese Sichtweisen beziehen sich auf die Vorstellung von Momentangeschwindigkeit und vom Grenzwertbegriff. $\mathrm{Zu}$ jeder Sichtweise gehören typische Vorstellungen in $\mathrm{F}$ und $\mathrm{m}$-Welt. Im Laufe der Bearbeitung der Teilaufgaben c) bis e) kann man gelegentlich beobachten, wie eine Schülerin oder ein Schüler einen Sichtweisenwechsel vornimmt. Solche Sichtweisenwechsel werden in den Transkriptanalysen in Kapitel 5 herausgearbeitet. Zu Beginn und am Ende der Partnerarbeitsphase hat diese Schülerin / dieser Schüler also verschiedene Vorstellungen bzw. Modelle von Momentangeschwindigkeit.

Der Aspekt a) des pragmatischen Merkmals wird besonders betont, wenn man wie Busse $^{49}$ versucht, das Konzept der subjektiven Erfahrungsbereiche (SEBe) nach Bauersfeld ${ }^{50}$ auf die Modellbildung zu übertragen. Die Denkwelten gehen in verschiedenen SEBen auf, welche die „...Gesamtheit des als subjektiv wichtig Erfahrenen und Verarbeiteten, einschließlich der Gefühle, der Körpererfahrung usw. also nicht nur der kognitiven Dimension“51 umfassen. $r$ und $m$-Welt als Teile von SEBen können nach Bauersfeld völlig getrennt voneinander vorliegen. Die Herstellung von Zusammenhängen zwischen den SEBen erfordert die Ausbildung eines dritten SEBs. „Die Bildung eines Modells kann man als Konstituierung eines dritten SEBs interpretieren, der den Blick auf das reale Problem einerseits und auf dessen Mathematisierung andererseits zulässt und diese beiden integriert."52 Diese Überlegungen sind für diese Untersuchung nicht uninteressant:

\footnotetext{
${ }^{49} \mathrm{vgl}$. [Busse 1999]

50 vgl. [Bauersfeld 1983]

51 [Bauersfeld 1983], S. 2

52 [Busse 1999], S. 244
} 
Zusammenhänge zwischen Gegenstandsbereichen können vermutlich nur auf der Denkwelt-Ebene hergestellt werden, insbesondere wenn die Gegenstandsbereiche so unterschiedlich sind wie in dieser Arbeit. Wenn also hier die Frage gestellt wird, welche Zusammenhänge Schülerinnen und Schüler zwischen Bewegungsvorgängen und Differenzialrechnung sehen, muss man zunächst versuchen, die Denkwelten der Schülerinnen und Schüler so gut es geht, offen zu legen. Aber: Denkwelten sind subjektiv und daher von Mensch zu Mensch verschieden (vgl. Aspekt a)). Nur unter der Prämisse, dass Denkwelten verschiedener Menschen von gewissen Alltagssituationen eine gewisse Ähnlichkeit aufweisen, kann diese Arbeit akzeptiert werden. Oevermann beschreibt diese Voraussetzung bezüglich seiner objektiven Hermeneutik in der Sozialforschung so:

\begin{abstract}
„Kriterium für die Gültigkeit der Auslegung dieser Sinnstrukturen sind genau jene Regeln, die in der Realität selbst an der Erzeugung der Sinnstrukturen beteiligt waren und über die der Interpret mehr oder weniger gut per Sozialisation in seiner gesellschaftlichen Lebenspraxis verfügt... Da die Sinnstrukturiertheit von sozialen Abläufen und Objektivationen regelgeleitetes Handeln zwingend voraussetzt, gründet sich die methodologische Geltungsbegründung von Interpretationen auf die Geltung von Regeln und auf deren naturwüchsige Existenz, zudem darauf, daß die Regeln, da von Naturgesetzen wesensmäßig unterschieden, einen von diesen unabhängigen, eigenständigen Anspruch auf Geltung erheben." ${ }^{53}$
\end{abstract}

Oevermann geht davon aus, dass Denkwelten als Sinnstrukturen eines Gegenstandbereichs (z.B. Bewegungsvorgänge) nach gewissen Regeln aufgebaut werden. Ziel seiner objektiven Hermeneutik ist, die Bedeutung einer Situation aufgrund geltender Regeln zu erfassen. Auch die Nutzung der in Kap. 2.3 vorgestellten Grounded Theory setzt eine gewisse Ähnlichkeit der Denkwelten bezüglich Bewegungsvorgängen voraus. Man kann in dieser Arbeit ein Indiz dafür sehen, dass diese Ähnlichkeiten auftreten: Gäbe es neben aller subjektiver Wahrnehmung und subjektiver kognitiver Verarbeitung diese Ähnlichkeiten nicht, hätten die in Kap. 4 vorgestellten Sichtweisen als Oberbegriffe für die Schülerinnen- und Schüler-Vorstellungen in beiden Denkwelten wahrscheinlich nicht entwickelt werden können. Die gefundenen Sichtweisen haben sich bei der Analyse und Einordnung aller Schülerinnen- und Schüler-

\footnotetext{
${ }^{53}$ [Oevermann 1983], S. 97
} 
Äußerungen bewährt, die sich auf das Wesen der Momentangeschwindigkeit und des Grenzwerts beziehen, sie „passen“ und „funktionieren“ (vgl. 2.3).

Bei der Interpretation der Transkripte hat sich gezeigt: Wenn Schülerinnen und Schüler versuchen, Zusammenhänge zwischen $r$ - und $m$-Welt herzustellen, kommt es aufgrund des Versuchs, Verbindungen aufzuzeigen, zwangsläufig dazu, dass an ihren Äußerungen nicht klar zu erkennen ist, ob diese der einen oder anderen Denkwelt zugeordnet werden können. Erst der Gesprächskontext zeigt an, welche Welt jeweils stärker betont wird.

\section{Die Frage nach einer Physik-Welt}

Bei Bewegungsaufgaben, Vorstellungen und Beschreibungen von Bewegungsvorgängen kann man die Frage nach einer Physik-Welt mit der zugehörigen Denkwelt ( $p$-Welt) stellen. Gegenstandsbereich des hier wichtigen Teils der Physik sind Bewegungsvorgänge. Das ist aber auch Gegenstandsbereich der r-Welt. Es wird nun gezeigt, eine $p$-Welt in dieser Arbeit kaum auszumachen ist:

- Der Begriff der Momentangeschwindigkeit als physikalischer Begriff ist hier zentral. Daneben gibt es aber auch die Alltagsbedeutung des Worts „Momentangeschwindigkeit“. Wie im vorhergehenden Abschnitt „Verkürzungsmerkmal“ angedeutet, unterscheiden sich die Begriffe der Momentangeschwindigkeit in der Physik (Geschwindigkeit = Tempo und Richtung; Repräsentation durch einen Vektor) und im Altag (Geschwindigkeit bei geradlinigen Bewegungen; Repräsentation durch eine Zahl) erheblich. Die Schülerinnen und Schüler verwenden „Momentangeschwindigkeit“ ausschließlich in der Alltagsbedeutung.

- Zur Beschreibung einer physikalischen Theorie wird oft eine mathematische Sprache verwendet, wie bei den Gesetzen der Bewe gungslehre, z.B. $S=v \cdot$ t. Physikalische Beschreibungen von Bewegungsvorgängen sind in ihrer äußeren Form von mathematischen Schreibweisen kaum zu unterscheiden. Kann aber die Verwendung mathematischer Objekte und Kalküle zur Beschreibung von Bewegungsvorgängen durch die Schülerinnen und Schüler bzw. der Wunsch sie zu nutzen, ein Zeichen da- 
für sein, dass sie sich gedanklich in der $p$-Welt aufhalten? - Die Schreibform $S=v \cdot t$ kann wohl erst der $p$-Welt zugeordnet werden, wenn unter S (Weg), v ((Momentan-) Geschwindigkeit) und $t$ (Zeit) die physikalische und nicht die alltagsweltliche Bedeutung verstanden wird. Dies ist bei den Schülerinnen und Schülern aber, wie gesagt, nicht der Fall. Die in der Aufgaben gegebene Weg-Zeit-Funktion wird von den innen wohl eher in der mWelt genutzt: In die Funktionsgleichung werden für $t$ Zahlen eingesetzt (Aufgabenteil a)), mit Hilfe der Funktionsgleichung werden "Formeln“ hergestellt (Aufgabenteil b)) und sie wird z.T. abgeleitet (Aufgabenteile d) und e)). Sie wird also eher für mathematische Tätigkeiten genutzt, vgl. „pragmatisches Merkmal, Aspekt b)“

- In den Transkripten äußern einige Schülerinnen und Schüler die Idee des Messens von Weg, Zeit und Geschwindigkeit „Ja, man müsste die ganze Zeit messen.“54 Messungen und Abschätzungen von Größen finden im Alltag ständig statt. Gemessen oder geschätzt wird etwas, von dem man eine Alltagsvorstellung hat. Messungen nach wissenschaftlichen Standards gehören aber auch zu den Tätigkeiten des Physikers, zumindest des Experimentalphysikers, der das Gemessene unter physikalischen Gesichtspunkten betrachtet. Da Schülerinnen und Schüler unter dem, was sie messen, die Alltagsbedeutung verstehen, kann man aufgrund dieser Tätigkeit alleine nicht auf ein gedankliches Verweilen in der $p$-Welt schließen.

- Spielt eine explizite $p$-Welt eine Rolle für die Schülerinnen und Schüler? Das Wort „Physik“ fällt nur sehr selten in den Gesprächen und wenn, kann vermuten, dass die Physik gerne als „Formel-Lieferant“ für die m-Welt gesehen wird:

„S: Wie gut, dass ich Physik habe.

A: Ja ok. Sag mir, wie man Geschwindigkeit errechnet!

S: Geschwindigkeit ist Weg pro Zeit. “55

„S: Momentangeschwindigkeit.

A: Ehm, das haben wir in Physik gemacht. [...] ... da musst du aber, glaube ich, eine andere Formel nehmen“ ${ }^{\circ 6}$

\footnotetext{
$54[03 ; 417]$

$55[04 ; 20-22]$

$56[13 ; 26-27]$
} 
Als Hilfe für die Suche nach der Antwort auf die Frage, ob es eine Momentangeschwindigkeit gibt, wird die Physik nur einmal herangezogen, als ein Schüler über den Energieerhaltungssatz zu einer Erkenntnis über das Wesen der Momentangeschwindigkeit gelangen will, vgl. [13]. Nur hier versuchen zwei Schüler, einen physikalischen Begriff in seiner physikalischen Bedeutung zu nutzen. Da die p-Welt in den Transkripten kaum ausgemacht werden kann, soll sie auch in dieser Arbeit nicht weiter beachtet werden.

\section{Die Frage nach einer Welt der Graphen}

In den Transkripten lässt sich aber eine weitere Denkwelt finden, die g-Welt der (Funktions-) Graphen und Kurven. Daher müsste man die m-Welt in eine g-Welt und eine algebraisch-arithmetische aWelt aufteilen. Die g-Welt lässt sich in 7 von 16 Transkripten finden, jedoch in nur zwei Transkripten ([01] und [04]) werden Überlegungen am Graphen herangezogen, um die Frage nach dem Wesen der Momentangeschwindigkeit oder dem Grenzwertbegriff zu klären.

- Für diese Untersuchung nicht uninteressant, aber weniger zentral ist die bloße Feststellung, dass die Steigung des Graphs der Geschwindigkeit des Radfahrers entspricht und umgekehrt, (vgl. [01], [04] und [16]), wobei aber in nur zwei Fällen nach einer Methode gefragt wird, mit der man die Steigung ermitteln kann. Zumeist werden Momentangeschwindigkeit bzw. Steigung des Graphs an einer Stelle als intuitiv existent angenommen.

- Einmal wird der Graph einer Treppenfunktion betrachtet, wo bei in den Funktionswerten Durchschnittsgeschwindigkeitswerte gesehen werden, vgl. [14]. Überlegungen bzgl. eines Grenzprozesses am Graphen werden nicht durchgeführt. Weiterhin wird zweimal die Möglichkeit geäußert, einen Graphen erstellen zu können, vgl. [07] und [10]. Der Graph selber wird dann aber nicht gezeichnet.

- Zuletzt sind noch zwei Schülerinnen zu nennen, die eine Art Leiste erstellen, an der sowohl Uhrzeiten als auch die Entfernungen eingetragen wurden, die der Radfahrer bis zu diesen Zeitpunkten zurückgelegt hatte, vgl. [11]. 
Festzustellen ist: In drei Fällen wird der Zusammenhang Steigung-Geschwindigkeit explizit genannt. Interessant für diese Untersuchung sind im Wesentlichen nur die beiden Fälle, in denen Überlegungen über Sekanten und Tangenten am Graphen herangezogen werden, um dem Wesen der Momentangeschwindigkeit und des Grenzwertbegriffs näher zu kommen. Daher spreche ich im Folgenden weiterhin von der $\mathrm{r}$ - und der $\mathrm{m}$-Welt, wobei die $\mathrm{m}$-Welt der a-Welt entspricht. Nur in den Fällen, in denen explizit zwischen a- und g-Welt unterschieden werden soll, komme ich auf diese zurück.

Fazit: In der weiteren Arbeit werden im Wesentlichen nur die $\mathrm{r}$ - und die $\mathrm{m}$-Welt eine Rolle spielen. Um Schülerinnen- und Schüler-Äußerungen einer Denkwelt zuordnen zu können, muss der gesamte Kontext des Gesprächs berücksichtigt werden. Dazu gehören neben der Sprache die verwendeten Bilder, Metaphern und Vorstellungen.

\subsection{Die Methode}

Ähnlich wie in der vorliegenden Untersuchung beschäftigt sich auch Rudolf vom Hofe mit Vorstellungen, die Schülerinnen und Schüler vom Grenzwertbegriff beim Ableiten entwickeln ${ }^{57}$. Vom Hofes primäres Anliegen besteht allerdings in verschiedenen Aspekten des Computereinsatzes im Mathematikunterricht, wie z.B. die Auswirkungen des Computereinsatzes auf Lehr- und Lernprozesse oder Kommunikationsprozesse. In seinem Schulunterricht hat vom Hofe u.a. Schülerinnen und Schüler mit Hilfe eines Funktionenplotters Untersuchungen zum Ableiten durchführen lassen. Auf einem Arbeitsblatt erhielten sie eine Funktionsgleichung und die Aufgabe, den Graphen und einige Sekanten durch einen Punkt des Graphs vom Computer erstellen zu lassen. Er verwendet für den Differenzialquotienten die Schreibweise $\lim _{h \rightarrow 0} \frac{f(x+h)-f(x)}{h}$ und fordert die Schülerinnen und Schüler auf, für $h$ verschiedene Werte einzusetzen. Der Computer erzeugt sofort den Graphen der Funktion $f$ und der Sekante durch $(x+h ; f(x+h))$ und $(x ; f(x))$. Den Schülerinnen und Schülern wird also die Möglichkeit gegeben, mit der Situation auf der symbolischen und auf der graphischen Darstellungsebene zu experimentieren. Bei diesem heuristischen Arbei-

${ }^{57}$ [vom Hofe 1998] 
ten versuchen die Schülerinnen und Schüler nun die Bedeutung des Parameters $\mathrm{h}$ herauszufinden. Vom Hofe hat einige Paare von Schülerinnen und Schülern gefilmt und anschließend die Videofilme transkribiert.

Im Unterschied zu vom Hofes Arbeit, bei der die Schülerinnen und Schüler zunächst eine Funktionsgleichung ohne Anwendungsbezug erhalten ${ }^{58}$, arbeiten die Schülerinnen und Schüler in der vorliegenden Untersuchung nicht an einer innermathe matische Aufgabe. Zudem liegt der Schwerpunkt dieser Arbeit nicht nur bei den Vorstellungen an sich, sondern darauf, wie sie sich beeinflussen. Ein wichtiger Aspekt ist bei vom Hofe die Visualisierung, die hier eine geringere Rolle spielt.

\section{Die empirische Methode und die Stoffdidaktik}

Wenn man so will, kann man zwei große Methodenkomplexe der deutschsprachigen Mathematikdidaktik gegenüberstellen: die Stoffdidaktik und die etwas jüngere empirische Unterrichtsforschung. Thomas Jahnke charakterisierte diese Komplexe 1998 so:

„Der Mathematikdidaktik in Deutschland wird schon seit mindestens zwei Jahrzehnten ihre Konzentration, ihre Zentrierung auf den Stoff vorgehalten und vorgeworfen. Der am häufigsten vergessene Existenzsatz der Mathematikdidaktik, heißt es zum Beispiel schon fast kalauerhaft, sei der Satz von der Existenz der Schülerinnen und Schüler. [... und der] Lehrer übrigens auch, sollte man hinzufügen, auch wenn das erst neuerdings wieder stärker betont wird." [...]

„Es gab und gibt auch eine Gegenreaktion auf die Stoffdidaktik, die darin besteht, deren Arbeiten und Ergebnisse zu ignorieren, etwa unter dem Motto, in der Schule, also im Mathematikunterricht gehe es wesentlich um Sozialformen und nicht um Gleichheitszeichen oder Termumformungen. So wie die Stoffdidaktik in Gefahr steht, die Schüler und den Unterricht aus dem Blickfeld zu verlieren, so kann solche Unterrichtsmethodik ihren Stoff verlieren. Eine offensichtlich unsinnige Alternative." 59

\footnotetext{
${ }_{58}^{5}$ Später wird die Funktion als Bakterien-Wachstumfunktion gedeutet.

59 [Jahnke 1998], S. 63; Jahnke macht darauf aufmerksam, dass die Bezeichnung „Stoffdidaktik“ zumeist von deren Kritikern verwendet wird.
} 
Die Ziele der Stoffdidaktik sind kurz gesagt die schulgerechte Aufbereitung mathematischer Inhalte. Die dazu genutzte Methode wird im Allgemeinen die Sachanalyse genannt. Die Ziele und Methoden der empirischen Unterrichtsforschung beschreibt Hölzl so: Es

„ist die methodisch kontrollierte Analyse von Transkripten (Verschriftlichungen), die aus dem audio- oder videodokumentierten Unterrichtsgeschehen hervorgehen." $[\ldots]$

„Ziel dieser interpretativen Analysen ist,

- das Unterrichtsgeschehen aus der Binnenperspektive der Beteiligten (Lernende, Lehrende) zu verstehen, und

- die Regelhaftigkeiten, denen die Interaktion zwischen den Beteiligten unterliegt, zu rekonstruieren. “60

\begin{tabular}{|c|c|c|}
\hline & Stoffdidaktik & $\begin{array}{c}\text { interpretative } \\
\text { Unterrichtsforschung }\end{array}$ \\
\hline \multirow{3}{*}{ Methode } & normativ (präskriptiv) & deskriptiv - interpretativ \\
& didaktisch orientierte Sachanalyse & $\begin{array}{c}\text { qualitativ-empirische Methoden } \\
\text { (Fallstudien: Interpretation von } \\
\text { Transkripten von Videos aufge- } \\
\text { zeichneter Unterrichtsstunden) }\end{array}$ \\
\hline \multirow{3}{*}{ Sicht } & $\begin{array}{c}\text { a priori } \\
\text { dient der Unterrichtsvorbereitung }\end{array}$ & $\begin{array}{c}\text { "Unterrichtsnachbereitung“ } \\
\text { macht Geschehenes verstehbar }\end{array}$ \\
& $\begin{array}{c}\text { Da eine Unterrichtsnachbereitung zugleich die Vorbereitung auf nachfolgenden Unterricht } \\
\text { und insbesondere auf die erneute Lehre desselben Inhalts in anderen Kursen sein sollte, } \\
\text { sind die obenstehenden Charakterisierungen als Betonung zu verstehen. }\end{array}$ \\
\hline geht aus & $\begin{array}{c}\text { vom mathematischen Inhalt } \\
\text { von der Schülerin / vom Schüler }\end{array}$ \\
\hline Grenzen & $\begin{array}{c}\text { der einzelne Schüler } \\
\text { die einzelne Schülerin }\end{array}$ & Verallgemeinerbarkeit \\
\hline
\end{tabular}

Tabelle 1: Stoffdidaktik und empirische Unterrichtsforschung (angelehnt an Überlegungen von D. Maczey in Siegen) 
Hölzl und vom Hofe unternehmen in ihren Untersuchungen einen Brückenschlag, mit dem sie die beiden methodischen Ansätze ${ }^{61}$ verbinden wollen. Hölzl erhebt den Anspruch, in seinen Untersuchungen wie folgt vorzugehen ${ }^{62}$ : Zunächst fordert er eine Sachanalyse der Problemaufgabe, um den mathematischen Inhalt zu explizieren. Dabei soll mindestens eine Lösungsvariante aufgezeigt werden. Dann ist anhand der Transkripte Stück für Stück das Geschehen aus der „Binnenperspektive der Handelnden" nachzuzeichnen. Dabei kommt es nicht auf eine Beurteilung des Verhaltens und der Äußerungen der Handelnden an, zu der die Sachanalyse als Maßstab herangezogen werden könnte. Vielmehr geht es um die „subjektive Schlüssigkeit“ des Problemlösevorgangs. In einem dritten Schritt soll der Problemlösevorgang unter „Einbezug sachlogischer und stoffdidaktischer Aspekte" analysiert werden. Am Ende steht der „Entwurf einer Gesamtdeutung“, zu dem auch die „subjektbezogene Rekonstruktion des Geschehens" aus sachlogischer Sicht beurteilt wird.

Ähnlich arbeitet vom Hofe: Nachdem er in seiner Dissertationsschrift ${ }^{63}$ anhand einer von G. Malle erstellten Fallstudie ${ }^{64}$ zeigt, dass nur normative, also sachanalytische Überlegungen zur Ergründung des Verhaltens einer Schülerin oder eines Schülers nicht ausreichen, betont er, dass ihm die Klärung der Vorstellungen des Schülers wichtig sind, die zu dem von inm eingeschlagenen Lösungsweg führen. So kommt er zu einer Vorgehensweise, bei der sich normative und deskriptive Methoden zu Vorschlägen konstruktiver Maßnahmen vereinigen können:

„Eine umfassende Erklärung der Schülerstrategie und der Mißverständnisse, die sich angesichts des vom Lehrer erwarteten bzw. vom Schüler eingeschlagenen Lösungsversuchs ergeben, bringt eine Analyse der normativ verwendeten Grundvorstellungen und der deskriptiv feststellbaren Individualvorstellungen, etwa unter den Leitfragen:

- Welche Grundvorstellungen sind zur Lösung des Problems aus Sicht des Lehrenden adäquat? (Normativer Aspekt)

- Welche individuellen Vorstellungen lassen sich im Lösungsversuch des Schülers erkennen? (Deskriptiver Aspekt)

\footnotetext{
${ }_{61}$ z.B. [Hölzl 1994], [vom Hofe 1998], [vom Hofe 1999]

62 vgl. [Hölzl 1994], S. 95 - 110

63 [vom Hofe 1995a]

64 vgl. [Malle 1988]
} 
Worauf sind etwaige Divergenzen zurückzuführen, und wie lassen sich diese beheben? (Konstruktiver Aspekt)"65

Unter diesen Leitfragen kann auch die vorliegende Arbeit betrachtet werden: In Kapitel 3 werden normative Aspekte der Begriffe „Momentangeschwindigkeit" und "Grenzwert des Differenzenquotienten“ betrachtet und Schülerinnen- und SchülerÄußerungen gegenübergestellt. In Kapitel 4 werden Ursachen möglicher Divergenzen aufgezeigt. Mit der Ermittlung von Ursachen für die Divergenzen treten zugleich Vorschläge zur Lösung derselben auf. Die gewonnenen Erkenntnisse werden in Kapitel 5 genutzt, um individuelle Vorstellungen einiger Schülerinnen und Schülern ausführlich vorzustellen und zu analysieren.

Die eigentliche Interpretation der Transkripte findet bei vom Hofe auf zwei Ebenen statt. Zunächst nähert er sich den Schüleräußerungen auf der Beschreibungsebene, um dann auf die Erklärungsebene überzugehen. Seine Analysen basieren auf dem Wechselspiel von deskriptiven und präskriptiven Betrachtungen, zu denen er die for genden Leitfragen heranzieht:

"- Nachzeichnen der subjektiven Schülerlogik. Welche Vorstellungen und Ideen werden in den Lösungsversuchen der Schüler deutlich? Inwieweit lassen sich Denkmuster bzw. Lösungsstrategien nachzeichnen?

- Vergleichende Einbeziehung präskriptiver Kategorien. Inwieweit lassen sich Denkprozesse der Lernenden mit vorhandenen didaktischen Begriffen und Modellen erfassen und erklären?"66

Nach diesen Leitfragen sollen auch die Transkripte der vorliegenden Arbeit interpretiert und analysiert werden. Als „präskriptive Kategorien“ bzgl. des Grenzwertbegriffs bei Folgen, Reihen und Funktionen sollen die hier in Kapitel 4 entwickelten Sichtweisen genauso herangezogen werden, wie diejenigen, die aus einer Anzahl von Veröffentlichungen bekannt sind ${ }^{67}$. Auch zu Schülerinnen- und Schüler-Vorstellungen der genannten Begriffe, gibt es einige Literatur ${ }^{68}$.

\footnotetext{
${ }_{66}^{65}$ [vom Hofe 1995a], S. 116 - 117, Hervorhebung wie im Original

66 [vom Hofe 1999], S. 196, Hervorhebungen wie im Original

67 Exemplarisch seinen hier [Bender 1991b], [Bivens 1986], [Blum/Törner 1983], [Hering 1989], [Kirsch 1960], [Weigand 1988], [Weigand 1993] genannt.

${ }^{68}$ Exemplarisch seinen hier [Bender 1991a], [Fischbein u.a. 1979], genannt. Eine Übersicht über die

Erforschung von Grundvorstellungen gibt [vom Hofe 1996].
} 
Vom Hofe schätzte 1998 diesen Ansatz so ein:

"Qualitative Forschungsmethoden haben sich in den letzten Jahren zunehmend auch im Bereich der Mathematikdidaktik etabliert und werden inzwischen neben den herkömmlichen quantitativen Methoden innerhalb der empirischen Unterrichtsforschung weitgehend akzeptiert. Ihr Einsatzbereich liegt insbesondere dort, wo sich das Erkenntnisinteresse auf ein Gebiet richtet, das sich nicht mit direkt ables baren metrischen Daten beschreiben lässt. Dies ist insbesondere der Fall, wenn es darum geht, individuelle Strategien und Vorstellungen bzw. subjektive Wirklichkeiten von Menschen möglichst genau zu erfassen bzw. zu rekonstruieren. [...] Ganz besonders bieten sich solche Forschungsmethoden an, wenn man sich aus deskriptiver Sicht dafür interessiert, ob Erklärungsmodelle, mit denen man Lern- bzw. Problemlöseprozesse beschreibt, tatsächlich in den Denkprozessen der Schüler die Rolle spielen, die man aus theoretischer Sicht vermutet.

Solche Fragestellungen sind für das Lehren und Lernen aller Schulstufen von groBem Interesse, auch und insbesondere für die Oberstufenanalysis. Dennoch sind qualitative Methoden bislang nur wenig in diesen Bereich vorgedrungen, ihre Vertreter befassen sich bislang im wesentlichen mit elementaren Fragestellungen aus der Grundschule und der Sekundarstufe I. “69

\section{Interpretative Forschung}

Nach den Herangehensweisen, wie vom Hofe und Hölzl beschrieben, versuchte ich zunächst auch, meine Arbeit durchzuführen: Mein Interessensgebiet und somit die zentralen mathematischen Begrifflichkeiten standen fest, und ich führte eine Sachanalyse durch. Wie sich später zeigte, sollte das eine erste und vorläufige sein. Dann entwickelte ich die Aufgabe und führte eine Voruntersuchung mit vier Probandenpaaren durch. Nach einer kleinen Modifizierung der Aufgabenstellung rekrutierte ich 16 Paare von Schülerinnen und Schülern für die Hauptuntersuchung, videographierte sie bei der Bearbeitung der Aufgabe und fertigte die Transkripte an.

Im Rückblick fällt mir an der nun folgenden Arbeitsphase auf, dass bei mir Beobachtungen aus den Transkripten zu neuen theoretischen Überlegungen führten, die sich wiederum auf die Sachanalyse auswirkten. Die dadurch neu entstandenen Erkenntnisse und Beobachtungsschwerpunkte veränderten wiederum den Blick auf die Tran-

$\overline{{ }^{69} \text { [vom Hofe 1998], S. } 258-259}$ 
skripte, u.s.f. Man kann sagen, dass die Sachanalyse sowie die Entwicklung der in Kapitel 4 vorgestellten Sichtweisen mit den Analysen der Transkripte eng verzahnt sind (anders als wie bei vom Hofe beschrieben). Diese Arbeitsweise ist in der explorativen und qualitativen Sozialforschung in Deutschland seit mindestens 30 Jahren unter dem Namen "gegenstandsbezogene Theorie“ bekannt und wird als Methode erforscht und angewendet. In den USA erlebte die Grounded Theory in den 40er und 50 er Jahren des letzten Jahrhunderts eine erste Hochzeit. Auch in der deutschsprachigen Mathe matikdidaktik wird diese Methode verwendet: Es lassen sich z.B. Ähnlichkeiten zwischen Voigts „empirisch gestützter Theoriebildung“70, Beck/Jungwirths „Deutungshypothesen“71, Oevermanns „objektiver Hermeneutik“72 und der Grounded Theory feststellen.

Glaser und Strauss charakterisierten 1979 diese Methode in einem Aufsatz ${ }^{73}$, dessen erster Abschnitt den Titel „Die Gleichzeitigkeit der Sammlung und Analyse von Daten“ trägt. In der Sozialforschung ist „die Gleichzeitigkeit von Sammlung und Analyse“ wörtlich zu nehmen, da sich die Forscher über längere Zeiträume in dem zu untersuchenden Milieu bewegen. Bei mir lagen alle Transkripte vor, dann erst begann ich sie mit den Augen des Forschers zu lesen und interessante Textstellen herauszusuchen. Wenn man darunter auch „Sammeln von Daten“ verstehen will, beschreiben Glaser und Strauss meine Herangehensweise an die Transkripte:

\begin{abstract}
„Ganz gleich, ob der Feldforscher zunächst noch sehr orientierungslos damit beginnt, alles, was er sieht, aufzuzeichnen, weil alles bedeutsam sein könnte, oder ob er mit einer genau definierten Zielsetzung ins Feld geht: seine Beobachtungen werden sehr rasch von Hypothesenbildungen begleitet sein. Wenn dieser Prozeß der Hypothesenbildung beginnt, kann der Forscher nicht mehr, selbst wenn er dies wünscht, ein passiver Empfänger von Eindrücken bleiben; er wird ganz automatisch dazu übergehen, aktiv solche Daten zu sammeln, die für die Entwicklung und Verifizierung seiner Hypothesenbildung bedeutsam sind. ${ }^{74}$
\end{abstract}

\footnotetext{
${ }^{70}$ In [Voigt 1984], Kap. 3.1.3 wird zwischen Theoriebildung durch Deduktion, Induktion und Abduktion unterschieden. Man kann Ähnlichkeiten zwischen abduktiven Theorien und Grounded Theories erkennen. In [Voigt 1996] werden auf Seite 393 die Abduktion und die „empirisch gestützte Theoriebildung" verbunden. Dadurch werden Parallelen zur Grounded Theory noch deutlicher.

${ }_{72}^{71}$ [Beck/Jungwirth 1999]

72 [Oevermann 1983]

73 [Glaser/Strauss 1979a]

74 [Glaser/Strauss 1979a], S. 92 - 93
} 
Bei der vorliegenden Arbeit stand die Zielsetzung von Anfang an fest: Das Augenmerk sollte auf die Vorstellungen zum Grenzwertbegriff gerichtet werden. Das Studieren der Transkripte ermöglichte eine Hypothesenbildung, die zur Ausarbeitung der besagten Sichtweisen führte. Auch das aktive Sammeln von Daten gab es: Es gibt Kopien der Transkripte, die ausschließlich nach Textstellen bzgl. der Sichtweisen durchgearbeitet wurden.

Ziel dieser Herangehensweise ist die Entwicklung von Hypothesen und Theorien aus Daten:

„Deutungshypothesen in der interpretativen Forschung zielen auf das „Verstehen“ der Phänomene;“ „Das erklärungsbedürftige Phänomen wird nicht aus mehr oder minder Allgemeinem hergeleitet, sondern es wird gleichsam umgekehrt versucht, neue Prinzipien zu entwickeln, unter deren Annahme dann das Phänomen plausibel erscheint.“ „Es werden „Typen“ gebildet - Konstruktionen, in denen ein Moment der Abstraktion enthalten ist, und die deswegen nicht unbedingt beschränkt sind auf die in die Untersuchung eingegangenen Daten." 75

Die besagten Sichtweisen wurden tatsächlich als Abstraktion aus Beobachtungen aus den Transkripten entwickelt. Die Schlüsselstellen für meine Erarbeitung der Sichtweisen stammen aus zwei verschiedenen Transkripten und werden in Kapitel 4 vorgestellt. - Über den Wert so entstandener Hypothesen und Theorien sagen Glaser und Strauss:

\footnotetext{
„Wie wir zu zeigen versuchen, ,paßt‘ nämlich eine solche Theorie auf empirische Situationen, und ist für Soziologen wie für Laien verständlich. Vor allem aber: sie funktioniert - sie liefert uns relevante Vorhersagen, Erklärungen, Interpretationen und Anwendungen!"

„Mit ,passen“ meinen wir, daß [gefundene] Kategorien leicht (nicht mit Gewalt) auf die untersuchten Daten anwendbar und durch se angezeigt sein müssen; mit ,funktionieren" meinen wir, daß sie in sinnvoller Weise für das untersuchte Verhalten relevant sind und es erklären können. ${ }^{“ 76}$
}

Es mag wie ein Ringschluss erscheinen, dass man aus Daten eine Theorie entwickelt, um dann zu sehen, dass die Daten zur Theorie passen. Dies ist aber keine Schwäche, sondern eine Stärke dieses Ansatzes. Schließlich müssen die beobach-

\footnotetext{
${ }_{76}^{7}$ [Beck/Jungwirth 1999], S. 232, S. 235, S. 243

76 [Glaser/Strauss 1979b], S. 63 und 64
} 
teten Phänomene nicht in vorgegebene Kategorien hinein gesehen werden. Zudem werden ja auch Forderungen an die Qualität der Theorie gestellt: Sie muss für das zu untersuchende Phänomen relevant sein und es erklären können.

Die Relevanz von Beobachtungen für eine Theorie kann durch die Hinzunahme von Vergleichsgruppen geprüft werden. Ähnlichkeiten und Unterschiede werden herausgearbeitet. Ähnliche Beobachtungen in verschiedenen Gruppen deuten auf Relevanz hin. In der vorliegenden Arbeit gab es 16 Vergleichsgruppen. Der Vergleich von Ähnlichkeiten und Unterschieden ermöglicht eine "generalisierende Analyse der Beziehungen zwischen den Kategorien, aus welcher dann Hypothesen folgen, die in die gegenstandsbezogene Theorie integriert werden“77. Dass eine so entwickelte Theorie diesen Ansprüchen genügen kann, begründen Glaser und Strauss weiterhin so:

\begin{abstract}
„Darüber hinaus lassen strategisch bemerkenswerte Ereignisse neue Kategorien und Hypothesen entstehen oder erzeugen Zweifel an der Tragfähigkeit bestimmter Kategorien oder stellen früher formulierte Hypothesen in Frage. Solche bemerkenswerten Ereignisse werden entweder sofort, wenn sie geschehen, analysiert; andernfalls tauchen sie immer wieder mit unerträglich werdender Hartnäckigkeit in der Erinnerung auf, bis sie systematisch bei der Anfertigung von Vermerken ausgewertet werden." ${ }^{78}$
\end{abstract}

Der Entwicklungsprozess einer Theorie wird als abgeschlossen betrachtet, wenn sich neue empirische Fälle unter die Theorie fassen lassen. Ein Indiz für eine Art Sättigung bei der Theoriebildung sehen Glaser und Strauss darin, dass dem Forscher aus diesem Grunde langweilig wird, er entdeckt nichts Neues mehr. Aus diesem Grund werden im 5. Kapitel dieser Arbeit auch nicht alle vorhandenen Transkripte und ihre Analysen vorgestellt. Vielmehr wurde versucht, an einigen Szenen einer Auswahl von Transkripten typische Denk- und Argumentationsweisen der Schülerinnen und Schüler aufzuzeigen. Die Entwicklung der Theorie, hier der Sichtweisen, wird durch Hinzunahmen von Beispielen verschiedener Transkripte illustriert und nachvollzogen.

\footnotetext{
${ }^{77}$ [Glaser/Strauss 1979a], S. 98

78 [Glaser/Strauss 1979a], S. 94
} 
Also kann man den Aufbau dieser Arbeit auch so verstehen: In Kapitel 3 findet eine Sachanalyse, in 4 eine Theorieentwicklung statt. Die Theoriebildung wird mittels Beispielen aus Transkripten durchgeführt und begründet. Die Transkriptanalysen in Kapitel 5 dienen dem empirischen Nachweis der Theorie. Sie zeigen, dass man mit den entwickelten Begriffen Vorstellungen der Schülerinnen und Schüler adäquat beschreiben und erklären kann, dass die Theorie passt und funktioniert.

\subsection{Rekrutierung der Probandinnen und Probanden}

Um einen Einblick in die oben genannten Vorstellungen in den Denkwelten und das Verhältnis der Denkwelten zueinander erhalten zu können, wurde 16 Paaren von Schülerinnen und Schülern aus verschiedenen Gymnasien die oben angeführte Aufgabe gestellt. Zwei Paare besuchten zum Zeitpunkt der Videoaufnahme die 10. Klasse, ein Paar die 11. Klasse, die restlichen 13 Paare die 12. Klasse. Fünf Paare belegten einen Leistungskurs, neun Paare einen Grundkurs. Es nahmen 23 Mädchen und neun Jungen teil.

Die Probandinnen und Probanden wurden aus verschiedenen Klassen bzw. Kursen in Mathematikstunden rekrutiert. Der Versuchsleiter stellte sich als angehender Mathematiklehrer vor, der z.Z. „in der Mathematikdidaktik an der Uni“ arbeitet und eine Untersuchung mit Schülerinnen und Schülern durchführen will. Er informierte die Schülerinnen und Schüler darüber, dass immer Paare von Probanden gesucht seien, und dass diese gemeinsam und nicht im Wettbewerb gegeneinander eine Aufgabe bearbeiten sollen. Auch wurde den Schülerinnen und Schülern verdeutlicht, dass weniger eine perfekte „Lösung“ von Interesse sei, sondern der Weg der Bearbeitung. So konnte auch begründet werden, dass die Probandinnen und Probanden bei der Arbeit gefilmt werden sollten. 


\section{Bemerkungen zum Geschwindigkeitsbegriff}

In diesem Kapitel soll der Geschwindigkeitsbegriff, insbesondere die Begriffe der Durchschnittsgeschwindigkeit und der Momentangeschwindigkeit unter verschiedenen Gesichtspunkten betrachtet werden. Dabei spielt die mathematische Beschreibung von Bewegungsvorgängen eine wichtige Rolle, insbesondere der Zusammenhang zwischen Weg-Zeit-Funktionen und Geschwindigkeits-Zeit-Funktionen. In Abschnitt 3.1 wird der alltägliche Geschwindigkeitsbegriff analysiert und mit dem physikalischen Geschwindigkeitsbegriff verglichen. Im zweiten Unterkapitel geht es um mathematische Beschreibungen von Bewegungsvorgängen. Zunächst werden zwei Sichtweisen bezüglich der Weg-Zeit-Funktion entwickelt. Anschließend findet sich eine Analyse des Begriffs der Momentangeschwindigkeit. Weiterhin werde ich zeigen, dass die bekanntesten (alltäglichen) Messinstrumente für die Momentangeschwindigkeit diese genau genommen gar nicht messen. Zuletzt stelle ich die Kriterien vor, die Schülerinnen und Schüler nutzen, wenn sie versuchen für sich zu entscheiden, ob es so etwas wie eine Momentangeschwindigkeit überhaupt gibt oder nicht.

\subsection{Durchschnittsgeschwindigkeit und Momentangeschwindigkeit als Altagsbegriffe}

Das, was man mit "Momentangeschwindigkeit" und "Durchschnittsgeschwindigkeit“ bezeichnet, taucht in Altagssituationen auf. Im Folgenden werden einige dieser Situationen aufgezeigt und mit dem Typ von Geschwindigkeit in Verbindung gebracht, der aus meiner Sicht stärker betont wird.

- „Geblitzt werden“: „Ich war so 63 (Sachen, Kilometer, km/h, ...) schnell.“ Gemeint ist die Momentangeschwindigkeit, mit der man zu dem Zeitpunkt unterwegs war, als man geblitzt wurde. Ob tatsächlich eine Momentangeschwindigkeit gemessen wurde, ist hier unwichtig, es kommt hier darauf an, als was der Wert angesehen wird. 
- Skispringen: Ist der Springer über den Schanzentisch gekommen, wird im Fernsehen oft eine Geschwindigkeit (z.B. 89,6 km/h) eingeblendet. Da der Springer, solange er sich auf der Schanze befindet, ständig an Geschwindigkeit zunimmt, wird man unter diesem Wert wohl die maximale Geschwindigkeit zum Zeitpunkt des Absprungs verstehen, also eine Momentangeschwindigkeit.

- Beim Tennis wird nach einem Aufschlag gelegentlich die Aufschlaggeschwindigkeit angezeigt. Auch diese versteht man als Momentangeschwindigkeit.

- Eisschnelllauf / 100m-Lauf / Radrennen / Formel 1: Am Ende des Wettkampfes werden nicht nur die Wettkampfzeiten (benötigte Zeit) angezeigt, sondern öfter auch Geschwindigkeitswerte. Damit sind Durchschnittsgeschwindigkeiten gemeint.

- Formel 1: "Fahrer A war der schnellste, weil er das Rennen gewonnen hat, Fahrer B hat die schnellste Runde hingelegt, und Fahrer C war der schnellste bei der TopSpeed-Messung." Hier liegt kein Widerspruch vor, da sich die Schnelligkeit bei Fahrer A auf die Durchschnittsgeschwindigkeit über das gesamte Rennen bezieht, bei Fahrer B auf die Durchschnittsgeschwindigkeit einer Rennrunde, und die Schnelligkeit bei Fahrer $\mathrm{C}$ auf eine Momentangeschwindigkeit bei der Top-SpeedMessung.

Man sieht, dass man sich zu beiden Begriffen lebensweltliche Situationen vorstellen kann. Beide Begriffe haben eine Alltagsbedeutung. In jedem der Beispiele kann die Geschwindigkeit durch eine Zahl beschrieben werden. Für einige Schülerinnen und Schüler kann dieser Wert auch negativ sein. Für sie entscheidet das Vorzeichen des Werts, ob der Radfahrer vorwärts oder rückwärts fährt ${ }^{79}$.

In der klassischen Bewegungslehre, bei der man relativistische und quantenphysikalische Überlegungen nicht berücksichtigt, wird die Momentangeschwindigkeit durch einen Vektor beschrieben. Der Fußpunkt des Vektors befindet sich gewöhnlich an dem Ort (im R³), an dem sich der beobachtete Körper gerade befindet. Die Richtung des Vektors gibt die Bewegungsrichtung an. In ihr versteckt sich das Vorzeichen, das die Schülerinnen und Schüler z.T. berücksichtigen. Dem Wert der Momentange-

\footnotetext{
${ }^{79}$ vgl. z.B. $[14 ; 136-137]$ und $[16 ; 184]$
} 
schwindigkeit entspricht die Länge des Vektors. Die Länge des Vektors wird in der klassischen Physik mit „Tempo“ bezeichnet. Dort spielt der Unterschied zwischen Geschwindigkeit und Tempo eine bedeutende Rolle: Jede Krafteinwirkung $(\neq 0)$ auf einen Körper muss eine Änderung seiner Geschwindigkeit (aber nicht zwangsläufig seines Tempos) bedeuten. Einem Gegenstand, den man mit gleichbleibender Kraft über dem Kopf schleudert, würde man im Alltagsverständnis eine konstante Geschwindigkeit zumessen. In der Tat hat er aufgrund der konstanten Winkelgeschwindigkeit bei festem Radius ein konstantes Tempo. Dadurch, dass man aber beim Schleudern ständig der Zentrifugalkraft des Steins entgegenwirken muss, übt man eine ständige Kraft $(\neq 0)$ auf den Stein aus. Diese Kraft hält den Stein auf der Kreisbahn, bewirkt also eine permanente Richtungsänderung bei gleichbleibendem Tempo.

In der vorliegenden Aufgabe spielt der Begriff der Momentangeschwindigkeit eine zentrale Rolle. Eigentlich müsste nach dem Momentantempo gefragt werden. Da aber die Schülerinnen und Schüler unter Geschwindigkeit das Tempo verstehen, spielen die Namen der Begriffe für die Vorstellungen von der lokalen Änderungsrate keine Rolle. Handbewegungen auf den Videos lassen vermuten, dass sich die Schülerinnen und Schüler geradlinige Bewegungen bei ihren Überlegungen vorstellen. Die Geradlinigkeit der Bewegung bedeutet die Vernachlässigung der Richtung der Bewegung. Auch diese Vereinfachung ändert nichts an den für diese Untersuchung relevanten Vorstellungen, die man von der Momentangeschwindigkeit als lokale Änderungsrate entwickeln kann. Eine geradlinige Bewegung, wie sie sich die Schülerinnen und Schüler wohl zumeist vorstellen, hat i.A. eine Orientierung, die einige Teilnehmerinnen und Teilnehmer mit Hilfe eines Vorzeichens des Geschwindigkeitswerts beschreiben wollen. Weiterhin reden die Schülerinnen und Schüler oft von positiver Momentangeschwindigkeit oder „Geschwindigkeit haben“, womit sie zum Ausdruck bringen wollen, dass sich der Radfahrer bewegt und nicht steht. Die außermathematische Einkleidung der verwendeten Aufgabe beschreibt eine Radtour des Radrennfahrers Jan Ullrich. Theoretisch könnte dieser auf seiner Radtour anhalten oder umdrehen, also die Orientierung der inm unterstellten geradlinigen Bewegung invertieren. Niemand erwartet das von einem Radrennfahrer. Aus diesem Grunde spielt auch die Orientierung in dieser Aufgabe keine Rolle, zumal sie wiederum keinen Ein- 
fluss auf die relevanten Vorstellungen vom Grenzwert und der Momentangeschwindigkeit hat.

Eine Vorabklärung der physikalischen Bedeutungen der Begriffe hätte bezüglich dieser Untersuchung zu unnötigen Präzisierungen geführt und hätte die Schülerinnen und Schülern wahrscheinlich verwirrt. Auch hier soll von nun an die ungerichtete Größe Tempo gemeint sein, wenn von Ge schwindigkeit die Rede ist.

Die allägliche Beobachtung von Körpern, die sich in einem Zeitraum ohne anzuhalten bewegen, legen es nahe, anzunehmen, dass die Körper in diesem Zeitraum zu jedem Zeitpunkt eine (positive) Geschwindigkeit haben. Denn hätten sie zu keinem Zeitpunkt positive Geschwindigkeit, würden sie sich nicht bewegen, wie ein Schüler bemerkt:

„Du kannst doch schreiben, dass [wenn] es keine Momentangeschwindigkeit geben würde, gibt's auch keine Durchschnittsgeschwindigkeit, weil dann wird er sich ja gar nicht bewegen. ${ }^{80}$

Weil natürliche Bewegungsvorgänge stetig verlaufen, lässt es die Alltagsvorstellung nicht zu, dass eine Bewegung nur zu gewissen zueinander diskreten Zeitpunkten keine Geschwindigkeit hat (Betrag der Geschwindigkeit ist Null): Es kann also z.B. nicht vorkommen, dass eine Bewegung mit einer konstanten positiven Geschwindigkeit über einen Zeitraum, an einigen zueinander diskreten Zeitpunkten die Momentangeschwindigkeit mit Wert Null hat. Den Fall, dass ein Körper ausrollt, anhält und sich dann sofort wieder in Bewegung setzt, lässt die Vorstellung eines natürlichen Bewegungsvorgangs selbstverständlich zu.

\subsection{Mathematische Beschreibungen von Geschwindigkeit}

Bei der Beschreibung von Bewegungsvorgängen sind physikalische Begriffe und mathematische Methoden kaum zu trennen. Bei der Bearbeitung der Aufgaben stet len sich viele Schülerinnen und Schüler einen über einen Zeitraum bewegten Körper

${ }^{80}[03 ; 281-283]$ 
vor. Ihnen ist intuitiv klar, dass seine Bewegung durch eine Weg-Zeit-Funktion beschrieben werden kann. Wäre das nicht der Fall, hätte innen die Interpretation der Bedeutung der Funktion f der Aufgabe wahrscheinlich deutlich mehr Probleme bereitet, als es in den Transkripten zu erkennen ist.

\section{Weg-Zeit- oder Orts-Zeit-Funktion?}

Grundsätzlich ist es möglich, die Funktion $\mathrm{f}$ der Aufgabe als Orts-Zeit-Funktion oder als Weg-Zeit-Funktion zu interpretieren. Eine Orts-Zeit-Funktion gibt an, an welchem Ort sich der Radfahrer zu einem Zeitpunkt befindet, eine Weg-Zeit-Funktion, wie weit er in dem Zeitraum gefahren ist. Die Interpretation hängt im Wesentlichen davon ab, ob man unter dem Symbol t einen Zeitpunkt oder eine Zeitdauer versteht. Die Aufgabenstellung (vgl. 2.1) selber lässt das offen:

In Aufgabenteil a) taucht das Symbol $t$ in beiden Bedeutungen auf: $t$ als Fahrdauer in Stunden (vgl. Wertetabelle), und $t$ als Zeitpunkt: Die Funktion $f$ gibt an, „wie weit er bis zu einem Zeitpunkt schon vorangekommen ist." Den Transkripten ist zu entnehmen, dass die Schülerinnen und Schüler unter $f(t)$ die Länge des Weges verstehen, der in diesem Zeitraum zurückgelegt wurde, bzw. der bis zu diesem Zeitpunkt zurückgelegt wurde. Für diejenigen, die die Ableitungsfunktion f' von $f$ betrachten, ist diese in jedem Fall die zu $f$ gehörige Geschwindigkeit-Zeit-Funktion. Auch hier sind wieder zwei Denkweisen möglich: Die Schülerinnen und Schüler überlegen in Aufgabenteil c), wie schnell der Radfahrer zum Zeitpunkt 16 Uhr der Radtour ist. Man könnte aber auch fragen, wie schnell der Radfahrer genau zwei Stunden nach dem Start war.

Folgende typische Formulierung zeigt, dass Schülerinnen und Schüler $t$ bezüglich $f$ als Zeitdauer, bezüglich $f^{\prime}$ als Zeitpunkt interpretieren: „A: Wir wolln ja wissen, wie schnell, wie schnell er fährt, um $16 \mathrm{Uhr}$, das ist mir klar. [...] J: [...] $16 \mathrm{Uhr}, \ldots$ in zwei Stunden hat er 89,6 Kilometer zurückgelegt. “81 Da der Start der Radtour um 14 Uhr ist, beschreibt 16 Uhr den Zeitpunkt 2 Stunden nach dem Start. Dieser Transkriptauszug zeigt, wie in der Alltagssprache Zeitdauern und Zeitpunkte anhand der Ein-

${ }^{81}$ [02, 77 - 80] ; aber auch z.B. [06 ; 258 - 259] 
heiten „Uhr“ und „Stunde“ unterschieden werden. Im Alltag bezeichnet man mit „16 Uhr 12 Minuten und 35 Sekunden“ einen Zeitpunkt, mit „16 Stunden 12 Minuten und 35 Sekunden“ eine Zeitdauer. Schülerinnen und Schüler, die in ihrer Bearbeitung tatsächlich f' berechnen, setzen für t dann ganz selbstverständlich 2 und nicht 16 ein ${ }^{82}$. Auf die Problematik, dass mit dem Symbol t in der Bewegungslehre sowohl Zeitpunkte, als auch Zeitdauern beschrieben werden, macht auch Arons aufmerksam. Dazu führt er in seinem Buch „A guide to introductory physics teaching“ insbesondere das Fallgesetz (1) und das Gesetz für die Durchschnittsgeschwindigkeit (2) an.
(1) $\mathrm{d}=\frac{1}{2} \mathrm{at}^{2}$
(2) $v=\frac{S}{t}$

Nach Arons wird beim Fallgesetz $t$ üblicherweise als Zeitpunkt, beim Gesetz für die Durchschnittsgeschwindigkeit als Zeitdauer gesehen. Er spricht sich dafür aus, $\mathrm{t}$ grundsätzlich als Zeitpunkt zu betrachten und bei dem Gesetz für die Durchschnittsgeschwindigkeit entsprechend $\Delta \mathrm{t}$ und $\Delta \mathrm{S}$ zu verwenden. Er empfiehlt, zu Beginn eines Lehrgangs geradlinige Bewegungsvorgänge zu betrachten und führt aus:

\footnotetext{
„The concept of acceleration is inextricably connected to instantaneous velocity. It is impossible to deal clearly and correctly with instantaneous quantities without discriminating between instants (or "clock readings") and time intervals. It is impossible to deal with back-and-forth motion without discriminating between positions, changes in position, and distances travelled by the body [...]. ${ }^{83}$
}

Seiner Erfahrung nach stellen sich Studienanfängerinnen und Studienanfänger die Momentangeschwindigkeit als eine (Durchschnitts-) Geschwindigkeit in einem sehr kurzem Zeitintervall vor. ${ }^{84}$ Vermutlich führt Arons diese Vorstellungen darauf zurück, dass seine Studierenden mit dem Symbol t einen Zeitraum verbinden, denn dann wird seine Forderung, t von Anfang an als Zeitpunkt zu betrachten, klar.

Diese Beobachtung kann an den vorliegenden Transkripten teilweise nachvollzogen werden. Eine für viele Schülerinnen und Schüler übliche Argumentation in dieser Untersuchung ist: Da zu einem $(\mathrm{t}=)$ Zeitpunkt keine Zeit vergeht, kann man keinen

\footnotetext{
82 vgl. z.B. [14] und [16]

83 [Arons 1990], S. 21

84 vgl. [Arons 1990], S. 22
} 
Weg zurücklegen, also keine Momentangeschwindigkeit haben. Auch die positive Formulierung dieser Aussage lässt sich finden: Bewegung findet nur in einem $(\mathrm{t}=)$ Zeitraum statt, daher kann man keine Momentangeschwindigkeit haben. Andererseits sagt die Alltagserfahrung, dass ein bewegter Körper zu je dem Zeitpunkt der Bewegung einen Momentangeschwindigkeit haben muss. Das Problem scheint zu sein, dass sich die Schülerinnen und Schüler einerseits eine Bewegung zu einem Zeitpunkt nicht vorstellen können, ihnen aber andererseits das beschriebene Aus weichen auf einen Zeitraum auch nicht weiter hilft. Dieses Phänomen soll später genauer untersucht werden, vgl. Kapitel 4. Weichen Schülerinnen und Schüler der Zeitpunktsvorstellung aus, kann der von Arons beschriebene Effekt auftreten:
„A: Wenn $t=0$ wird, (VL: Ja.) aber $t$ wird ja nicht $=0$.
$[\ldots]$
S: Also kriegt man einen ganz knapp davon abweichenden Näherungswert.

A: Grenzwert eben.“85

Ob die Doppeldeutigkeit von $t$ (alleinige) Ursache für dieses Phänomen ist, kann meines Erachtens aus den Transkripten nicht gefolgert werden: Z.B. betrachtet besagter Schüler A durchaus auch Zeitpunkte und Orte, bezüglich derer er die Länge einer Ortsänderung berechnet:

\footnotetext{
„Ehm, ja. Muss man, ... den Punkt, äh, $S_{2}$ minus den Punkt $S_{1}$ nehmen. [...] Genauso mit dem Zeitpunkt." ${ }^{86}$
}

Dennoch lehnt er mit der oben genannten Argumentation die Existenz einer ganz genauen Momentangeschwindigkeit ab.

Betrachtet man $t$ als Zeitpunkt, schlägt Arons vor, $f$ als Orts-Zeit-Funktion zu betrachten und $f(t)$ als Ort anzusehen. Ein zwischen zwei Zeitpunkten zurückgelegter Weg muss dann als Ortsänderung gesehen werden:

"One can, for example, start with a rolling ball or moving cart on the laboratory table; make (or imagine) a "flash picture" that shows the object at uniform time intervals; place a scale behind the object; lead the students to see that the

\footnotetext{
85 [13; $371-375]$

86 [13; 34 - 35]
} 
numbers on the scale do not represent distances travelled by the object; that, as distances they are distances from an arbitrary origin at which the object may never have been located; that it takes two such numbers to give information about a change of position within a specified time interval; that we give such numbers the name "position numbers"

Der Betrachtung von Orten mittels Vektoren im $\mathrm{R}^{3}$ geht Arons zu Beginn seiner Lehrgänge (sowohl in seinem Didaktik-Buch wie in seinem Lehrbuch zur Physik) aus dem Weg. Er reduziert die Komplexität, indem er zunächst nur "back-and-forth motions“ zulässt: Er betrachtet geradlinige Bewegungen, „a motion of a particle along our reference line“ ${ }^{\text {88, }}$, die eine Einheitsstrecke („scale“, s.o.) enthält. Zu jedem Zeitpunkt befindet sich der Körper an einem Punkt der Geraden. Auf dem eindimensionalem Objekt "Gerade" wird durch $f(t)$ genau ein Ort beschrieben. Ähnliche Vorstellungen sind bei den Schülerinnen und Schülern dieser Untersuchung zu finden. Z.B. zeichnet sich ein Probandenpaar eine Art "reference line“ auf. Sie enthält sowohl Orts- als auch Zeitangaben, vgl. Abbildung 3.

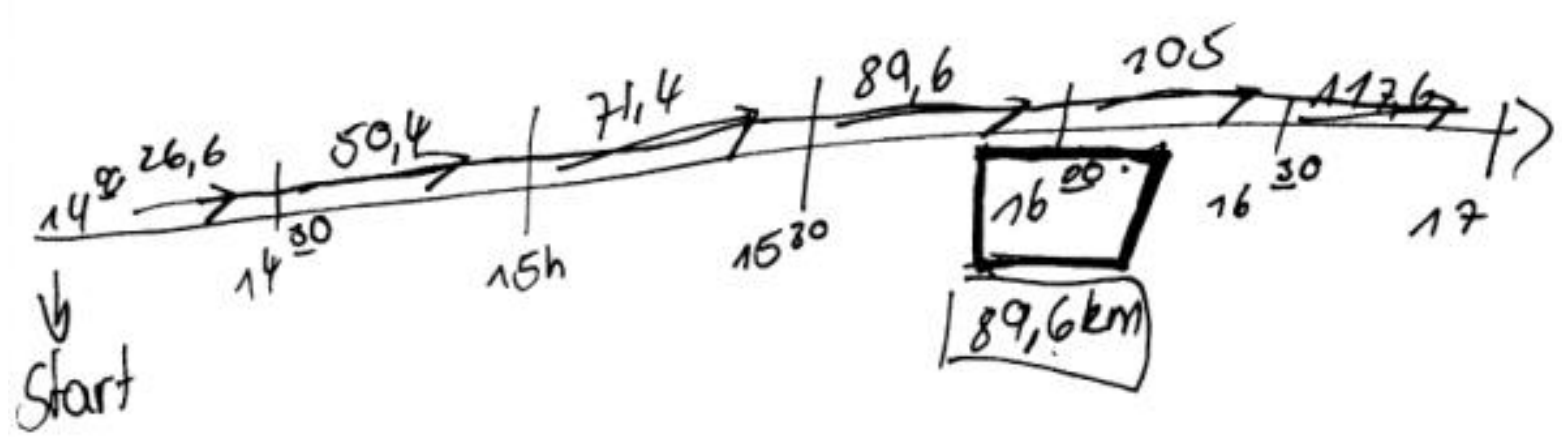

Abbildung 3 : Skizze aus Transkript 11, Bearbeitung zu Aufgabenteil c)

Ihrem Gespräch ist zu entnehmen, dass sich die beiden Schülerinnen gedanklich an dem Ort aufstellen, an dem der Radfahrer um 16 Uhr sein wird. Dann stellen sie sich vor, „wie Jan Ullrich nun angeradelt kommt“89. - Wenn die Route der Radtour feststeht, benötigt man keine geradlinige Bewegung, denn $f(t)$ kann als der Ort auf der Route gesehen werden, der auf dieser $f(t)$ Kilometer vom Start entfernt ist. Jedoch

\footnotetext{
87 [Arons 1990], S. 22

88 [Arons 1965], S. 3

$89[11,291]$
} 
lassen Handbewegungen einiger Schülerinnen und Schüler auf den Videos ${ }^{90}$ vermuten, dass diese sich auch geradlinige Bewegungen vorstellen. Vektorielle Repräsentationen von Orten sind in der vorliegenden Aufgabe nicht beabsichtigt und wurden von den Schülerinnen und Schülern auch nicht gezeigt.

Betrachtet man $\mathrm{t}$ als Zeitpunkt und $\mathrm{S}$ bzw. $\mathrm{s}(\mathrm{t})$ als Ort, also eine Orts-Zeit-Funktion, kann das Gesetz für die Durchschnittsgeschwindigkeit $v=\frac{S}{t}$ nicht mehr ohne Zusatzüberlegungen gelesen werden, da der Quotient aus einem Ort und einem Zeitpunkt nur schwerlich interpretierbar ist. Besser sollte man $v=\frac{\ddot{A} S}{A \ddot{t}}$ schreiben. Die Aufschlüsselung $v=\frac{\Delta S}{\Delta t}=\frac{S_{2}-S_{1}}{t_{2}-t_{1}}$ ist jedoch immer noch problematisch: Was soll z.B. unter der Differenz von zwei Orten verstanden werden? Erst wenn man wie Arons einen „arbitrary origin“ (s.o.), also einen willkürlich wählbaren Bezugspunkt in der Zeit to und den zugehörigen örtlichen Bezugspunkt $S_{0}$ hinzunimmt, kann man Weglängen und Zeitdauern notieren, da Orts-Differenzen bzgl. des Bezugszeitpunkts als Längen gesehen werden: $v=\frac{\left(S_{2}-S_{0}\right)-\left(S_{1}-S_{0}\right)}{\left(t_{2}-t_{0}\right)-\left(t_{1}-t_{0}\right)}$. to und $S_{0}$ sind in der vorliegenden Aufgabe sinnvoller Weise 14 Uhr und der Startort der Radtour. Betrachtet man den Raum seiner Lebens-Umwelt als Punktmenge mit Koordinatensystem, kommt durch die Möglichkeit der freien Wahl des Bezugspunkts nichts anderes als die Möglichkeit der freien Wahl des Ursprungs des Koordinatensystems zum Ausdruck.

Auch wenn man eine Weg-Zeit-Funktion betrachtet, benötigt man einen Bezugspunkt $S_{0}$ : Werden $S_{1}$ und $S_{2}$ in der Gleichung $v=\frac{S_{2}-S_{1}}{t_{2}-t_{1}}$ als Längen aufgefasst, kann man nach einem Startort der Bewegung fragen, bzgl. dessen die Entfernungen angegeben werden.

${ }^{90}$ z.B. [06, 190], [14, 170] 
Die Transkripte zeigen, dass die Interpretation von $S$ und t offensichtlich davon abhängig ist, was die Schülerinnen und Schüler (gedanklich) zu tun beabsichtigen ${ }^{91}$ : In Rechnungen ist es angenehmer, Zeitdauern und Entfernungen zu betrachten, bei Vorstellungen in der $r$-Welt werden Orte (an denen man sich gedanklich aufstellt) und Zeitpunkte (zu denen der Radfahrer irgendwo ist) bevorzugt. Bei (gedanklichen) Messvorgängen scheinen sich die Interpretationen zu treffen: Mit einem Maßband misst man die Entfernung $S_{2}-S_{1}$ zwischen zwei Orten $S_{1}$ und $S_{2}$.

Betrachtet man $t$ als Zeitpunkt und $f(t)$ als Ort, muss man konsequenterweise von einer Orts-Zeit-Funktion und nicht von einer Weg-Zeit-Funktion sprechen. Entsprechend ist eine Durchschnittsgeschwindigkeit als Orts-Änderungs-Rate, eine Momentangeschwindigkeit als lokale Orts-Änderungs-Rate zu sehen. Die Schülerinnen und Schüler interpretieren bei der Bearbeitung der Aufgabe $f$ jedoch zumeist als WegZeit-Funktion mit t als Zeitdauer. Unter Momentangeschwindigkeit verstehen sie fast immer, „wie viel er genau um 16 Uhr fährt“92, wobei t also als Zeitpunkt gesehen wird. Aus diesem Grund soll im Folgenden von der Weg-Zeit-Funktion die Rede sein, aber auch von den (lokalen) Orts-Änderungs-Raten.

Die sechs in dieser Diskussion zentralen Begriffe können wie folgt gegenübergestellt werden:

$$
\begin{array}{rrl}
\text { Ort } & \text { vs. } & \text { Weglänge, Strecke, Entfernung } \\
\text { Zeitpunkt } & \text { vs. } & \text { Zeitraum, Zeitdauer } \\
\text { Momentangeschwindigkeit } & \text { vs. } & \text { Durchschnittsgeschwindigkeit }
\end{array}
$$

\section{Die Momentangeschwindigkeit}

Wie in der Aufgabe soll jetzt als Information über die Bewegung nur eine Weg-ZeitFunktion s zur Verfügung stehen. Dann kann man die Orts-Änderungs-Rate des Körpers, also seine Durchschnittsgeschwindigkeit wie üblich berechnen:

\footnotetext{
${ }^{91}$ Dies ist ein schönes Beispiel dafür, dass verschiedene Modelle einer Situation in Abhängigkeit von der geplanten Operation gebildet werden, vgl. 2.2.

$92[11 ; 263]$
} 
„Ortsänderung / zugehörige Zeitdauer“:

$$
\frac{s\left(t_{2}\right)-s\left(t_{1}\right)}{t_{2}-t_{1}}
$$

Eine Bewegung wird aber durch die Angabe von $s\left(t_{1}\right)$ und $s\left(t_{2}\right)$, sowie $t_{1}$ und $t_{2}$ nicht vollständig beschrieben. Auch die Hinzunahme der Durchschnittsgeschwindigkeit liefert nicht alle Informationen, die man über eine Bewegung erlangen kann. Z.B. lässt sich so nicht feststellen, ob sich der Körper zu Beginn der Bewegung schnell oder langsam bewegt.

Zu jedem Zeitpunkt der Bewegung befindet sich ein solcher Körper an einem Ort zwischen Start- und Zielort. Zu jedem Zeitpunkt, also an jedem Ort hat er eine positive Momentangeschwindigkeit oder positive lokale Ortsänderungsrate. Bei gegebener Weg-Zeit-Funktion $s=s(t)$, kann die Momentangeschwindigkeit zum Zeitpunkt to mit Hilfe der Infinitesimalrechnung berechnet werden:

$$
\lim _{t \rightarrow t_{0}}\left(\frac{s(t)-s\left(t_{0}\right)}{t-t_{0}}\right)
$$

Bei einer linearen Weg-Zeit-Funktion kommt man auch ohne Infinitesimalrechnung aus, da sich der Körper mit konstanter Geschwindigkeit bewegt, die Durchschnittsgeschwindigkeit also denselben Wert hat, wie die Momentangeschwindigkeiten zu allen Zeitpunkten der Bewegung. Ein Körper, der über einen Zeitraum unbewegt bleibt, hat in diesem Zeitraum eine Durchschnittsgeschwindigkeit vom Wert Null. Ebenso haben seine Momentangeschwindigkeiten zu jedem Zeitpunkt des Zeitraums den Wert Null. Im Alltag wird hier oft formuliert, dass der Körper in diesem Fall keine Geschwindigkeit hat.

Eine Weg-Zeit-Funktion liefert hinreichend viel Informationen, um Durchschnittsgeschwindigkeiten $\frac{s\left(t_{2}\right)-s\left(t_{1}\right)}{t_{2}-t_{1}}$ berechnen zu können. Das bereitet Schülerinnen und Schüler (ab 10. Jahrgangsstufe wie in dieser Untersuchung) nur in den seltensten Fällen Probleme, zumal für die Berechnung nur elementare Arithmetik benötigt wird. Oft sind $t_{1}=0$ und $s\left(t_{1}\right)=0$, so dass die Differenzenbildung nicht durchgeführt werden muss. Wie die Einheiten z.B. „km/h“ bzw. „m/s“ anzeigen, geben (Durch- 
schnitts-) Geschwindigkeiten ein Verhältnis an. Damit sie einfach vergleichbar sind, werden gewöhnlich gleichnamige Nenner gewählt, in der Regel ist der Betrag des Nenners sogar gleich Eins. Somit brauchen beim Vergleich nur noch die Zähler betrachtet werden, was sich in der letztlich unpräzisen Alltagssprache ,ich war 160 Kilometer schnell“ niederschlägt. Wählt man die Weglängen als Maß für die Geschwindigkeit, kann man die Vorstellung des Verhältnisses umgehen. Der Quotient als solcher wird vielleicht gar nicht mehr wahrgenommen, da aufgrund der Eins im Nenner gar nicht gerechnet werden muss. Weglänge und Durchschnittsgeschwindigkeit werden identifiziert. Diese Identifikation wurde eine Zeit lang auf Verkehrsschildern genutzt, die die zulässige Höchstgeschwindigkeit (= maximale Momentangeschwindigkeit) begrenzten, vgl. Abbildung 4. Heute wird die Einheit „km“ nicht mehr auf die Schilder aufgebracht.

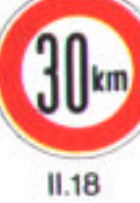

Abbildung 4: Identifikation von Weglänge und Momentangeschwindigkeit ${ }^{93}$

Die Geschwindigkeitsbegrenzung $30 \mathrm{~km} / \mathrm{h}$ bedeutet nicht, dass durchschnittlich maximal $30 \mathrm{~km} / \mathrm{h}$ gefahren werden dürfen, sondern, dass zu jedem Zeitpunkt die maximale Geschwindigkeit $30 \mathrm{~km} / \mathrm{h}$ betragen darf, dass also eine konstante Geschwindigkeit von maximal $30 \mathrm{~km} / \mathrm{h}$ erlaubt ist. Interessant ist, dass sowohl die Durchschnittsgeschwindigkeit als auch die Momentangeschwindigkeit auf die konstante Geschwindigkeit zurückgeführt werden können, also auf den trivialen Bewegungsvorgang: Durchschnittsgeschwindigkeit $30 \mathrm{~km} / \mathrm{h}$ bedeutet, dass, wenn man die ganze Zeit gleich schnell gewesen wäre, jede Stunde $30 \mathrm{~km}$ zurückgelegt hätte. Momentangeschwindigkeit $30 \mathrm{~km} / \mathrm{h}$ bedeutet, dass, wenn man die ganze Zeit so schnell fährt, in einer Stunde $30 \mathrm{~km}$ zurücklegt. In diesem Sinne ist die konstante Geschwindigkeit grundlegend. ${ }^{94}$

\footnotetext{
${ }_{93}$ [Duden 1984], S. 577

${ }^{94}$ Fragt man allerdings weiter, was bei der konstanten Geschwindigkeit "immer gleich schnell sein“ bedeuten soll, wird man wohl als Antwort erhalten, dass man zu jedem Zeitpunkt gleich schnell ist (immer $=$ zu jedem Zeitpunkt). Hier deutet sich an, dass die Begriffe der Momentangeschwindigkeit,
} 
Soll aus einer Weg-Zeit-Funktion die Momentangeschwindigkeit ermittelt werden, treten bei Schülerinnen und Schüler oft Probleme auf: Im Vergleich zu der elementaren Arithmetik werden die Infi nite simalrechnung und insbesondere Grenzprozesse oft als unnatürlich und schwer durchschaubar angesehen. Unterstützung sollen die Schülerinnen und Schüler u.a. von Grundvorstellungen für das Ableiten, z.B. die „lokale Änderungsrate" erhalten. Wie die Transkripte zeigen, bereitet aber die Momentangeschwindigkeit als lokale Orts-Änderungs-Rate selber bereits große Probleme. Oft betrachten Schülerinnen und Schüler Zähler und Nenner des Differenzen- und des Differenzialquotienten getrennt. Es ist natürlich richtig, dass zu einem Zeitpunkt keine Ortsänderung stattfindet, da keine Zeit vergeht. Daraus schließen einige Schüle rinnen und Schüler, dass es auch keine (positive) lokale Orts-Änderungs-Rate geben kann, dass es also in diesem Sinne keine Momentangeschwindigkeit geben kann. Bei Alexander klingt das so:

„Genau, weil in dem Augenblick keine Zeit vergeht. So. Und äh, theoretisch bewegt sich das Auto zu diesem Zeitpunkt nicht vorwärts. Sondern genau zu dieser Zeit bleibt es ja genau da stehen, wo ich hin gucke. [...] Ne Sekunde später ist das Auto woanders. Und dann kann ich den Weg, dann habe ich einmal den Weg, den Weg, den es zurückgelegt hat und die Zeit, und daraus ich irgendwie da die Geschwindigkeit berechnen. Also hat ne Geschwindigkeit, oder ne Beschleunigung oder wie auch immer, oder eine Verzögerung in diesem Zeitraum bestanden. [...] Aber genau zu dem Augenblick, wo ich kucke [...], ist es für mich gleich $0 .{ }^{\prime 95}$

Alexanders Argumentation zeigt das 0/0-Problem in der FWelt. Positive Geschwindigkeiten sind für inn nur über einen Zeitraum vorhanden. Er scheint nicht in der Lage zu sein, den Zeitpunkt innerhalb des Zeitraums der Bewegung zu sehen. Bezüglich des Zeitraums kann er aber nur Aussagen über die Durchschnittsgeschwindigkeit treffen, da sich die Geschwindigkeit des Radfahrers ständig ändern kann, wie andere Schülerinnen und Schüler bemerken ${ }^{96}$. Der "Moment“ wird bedeutungslos, daher kann es auch keine Momentangeschwindigkeit geben. Das steht aber im Gegensatz zur Altagsvorstellung dieser Größe, nach der ein bewegter Körper zu jedem Zeitpunkt der Bewegung eine positive Momentangeschwindigkeit hat. Im Tran-

der Durchschnittsgeschwindigkeit und der konstanten Geschwindigkeit einen Begriffskomplex bilden, dessen Teilbegriffe kaum getrennt voneinander verstanden werden können.

${ }_{95}[13 ; 245-253]$

96 vgl. z.B. [09; 86] und [12; 63 und 78] 
skript 06 kann man zwei Schülerinnen beobachten, die in genau diesen Konflikt geraten.

Im mathematischen Wortgebrauch stellt sich das 0/0-Problem so dar: Obwohl die lokale Ortsänderung und die lokale Zeitänderung jeweils den Wert Null haben, kann es zu dem Zeitpunkt eine positive lokale Orts-Änderungs-Rate geben.

Da sowohl Durchschnitts- als auch Momentangeschwindigkeit Verhältnisse angeben, dürfen Zähler und Nenner nicht getrennt betrachtet werden, jedenfalls nicht beim Grenzprozess. Also sind auch nicht zwei getrennte Grenzprozesse für eine Zählerund eine Nennerfolge zu betrachten, sondern der Grenzwert der Folge der Differenzenquotienten ist gesucht. Die getrennte Betrachtung kann eine Ursache für das 0/0Problem in der m-Welt sein, wie der folgende Dialog zeigt:
„A: Wenn $\mathrm{t}=0$ wird, (VL: Ja.) aber $\mathrm{t}$ wird ja nicht $=0$.
VL: Wenn $t=0$ wird, also einsetzen darfst du das nicht, dann hast du ja 0/0. (A: Ja, ja.)
S: Also kriegt man einen ganz knapp davon abweichenden Näherungswert.
A: Grenzwert eben.“97

Eine Folge von Alexanders Vorstellung ist, dass ein Grenzwert als etwas „unend lich Genaues, aber nicht ganz Genaues" angesehen wird, da man ja nicht 0/0 rechnen kann. An dem Transkriptauszug kann man zudem erkennen, dass nicht die oben genannte Doppeldeutigkeit des Symbols t für Alexanders Vorstellung verantwortlich ist. Bereits Newton unterschied sorgfältig zwischen "letzten Größen" und "letzten Verhältnissen“, vgl. Kap. 1.2.

Betrachtet man einen bewegten Körper ist intuitiv klar, dass es neben einer WegZeit-Funktion auch eine Geschwindigkeit-Zeit-Funktion geben muss, die zu jedem Zeitpunkt die Momentangeschwindigkeit des Körpers angibt. So eine Funktion taucht in der Alltagswelt z.B. bei Fahrtenschreibern von LKW auf. Hier tritt die Vorstellung der Momentangeschwindigkeit als lokale Orts-Änderungs-Rate in den Hintergrund. Da das Augenmerk meiner Untersuchung auf den Vorstellungen liegt, die Schülerinnen und Schüler vom Grenzwertbegriff beim Ableiten entwickeln, ist die Betrachtung

${ }_{97}[13 ; 371$ - 375]; $A$ = Alexander, $S=$ Sebastian, $V L=$ Versuchsleiter 
der Geschwindigkeit-Zeit-Funktion für mich zweitrangig. Dennoch ist es auch interessant zu sehen, wie z.B. ein Schüler der Jahrgangsstufe 10 in naiver Weise die Idee des Integrals als verallgemeinerten Mittelwert entwickelt, indem er die Durchschnittsgeschwindigkeit als verallgemeinerten Mittelwert der Momentangeschwindigkeiten sieht: $v=\frac{1}{t_{2}-t_{1}} \int_{t_{1}}^{t_{2}} v(t) d t$. Zur Erinnerung noch einmal das Zitat von oben:

„Du kannst doch schreiben, dass es keine Momentangeschwindigkeit geben würde, gibt's auch keine Durchschnittsgeschwindigkeit, weil dann wird er sich ja gar nicht bewegen." ${ }^{98}$

Oder anders formuliert: Wenn man sich zu keinem Zeitpunkt bewegt, bewegt man sich auch in dem Zeitraum, den die Zeitpunkte bilden, nicht. Diese Formulierung verweist auf die viel grundsätzlichere erkenntnistheoretische Diskussion über die Topologie des Kontinuums, auf die in Kapitel 4.5 eingegangen wird.

Ist eine Geschwindigkeit-Zeit-Funktion gegeben, bereitet es Schülerinnen und Schülern wohl keine Probleme, Momentangeschwindigkeiten als Funktionswerte zu berechnen. Im Gegensatz dazu wird nun die Berechnung von Durchschnittsgeschwindigkeiten wohl als schwer empfunden, da infinitesimale Mathematik benötigt wird.

Die Beschreibung von Bewegungsvorgängen durch Geschwindigkeit-Zeit-Funktionen (wie schnell man gerade ist) und Weg-Zeit-Funktionen (wo man gerade ist, bzw. wie weit man schon gefahren ist) lehnen sich eng an Alltagserfahrungen an und bereiten im Alltag keine Probleme. Das zeigen auch die Transkripte, in denen sich niemand über die gegebene Weg-Zeit-Funktion verwundert. Will man aus einer Weg-ZeitFunktion die Momentangeschwindigkeit ermitteln, rückt die Idee der lokalen Orts-Änderungs-Rate ins Zentrum der Aufmerksamkeit. Diese wird als unnatürlich empfunden. Es treten also Probleme auf, wenn man sich die Bewegung eines Körper nur zu einem Zeitpunkt und nicht über einen Zeitraum vorstellt. Da man keine Informationen über den Körper bezüglich seiner Lage vor oder nach diesem Zeitpunkt hat, kann man nicht entscheiden, ob dieser Körper ruht oder in Bewegung ist. Ein Foto als Momentaufnahme gibt keine Information über eine Bewegung. Die Vorstellung eines

${ }^{98}[03 ; 281-283]$ 
Zeitpunkts im Sinne eines Fotos verbietet die Vorstellung von Bewegung zu diesem Zeitpunkt und damit auch die Vorstellung von Geschwindigkeit zu diesem Zeitpunkt. Selbst wenn man wüsste, dass sich das Auto zum Zeitpunkt der Aufnahme bewegte, könnte man dem Foto die zugehörige Geschwindigkeit nicht entnehmen. („Verwischte" Fotos z.B. einer Radarfalle sollen hier nicht berücksichtigt werden, siehe unten "Messbarkeit von Momentangeschwindigkeit“.) Die Vorstellung einer Bewegung oder einer Geschwindigkeit zu einem Zeitpunkt erscheint sinnlos, bei der Bewegung zu Recht, bei der Geschwindigkeit zu Unrecht: Auch wenn zu einem Zeitpunkt keine lokale Orts-Änderung stattfindet, kann ein Körper eine positive lokale Orts-Änderungsrate haben. - Betrachtet man die Weg-Zeit-Funktion zu einem bestimmten Zeitpunkt, so weiß man, an welchem Ort sich der Körper zu diesem Zeitpunkt befindet, aber nicht, wie er dort hin gelangt ist. - Man kann sich Bewegungsvorgänge über Zeiträume vorstellen. Wenn man die Vorstellung von Bewegung zu einem Zeitpunkt ablehnt, werden Zeitpunkte bedeutungslos. Man kann nur noch Aussagen über Wegstrecken und Durchschnittsgeschwindigkeiten treffen.

Fazit: Ob im Mathematikunterricht die Berechnung und Vorstellungen von der Durchschnitts- und der Momentangeschwindigkeit als schwer oder leicht empfunden werden hängt davon $a b$, welcher funktionale Zugang vorgegeben ist, eine Weg-ZeitFunktion oder eine Geschwindigkeit-Zeit-Funktion.

Anhand von Transkriptauszügen wurden hier Schülerinnen- und Schüler-Vorstellungen vom Grenzwertbegriff und von Geschwindigkeiten (insbesondere Momentangeschwindigkeit) als Phänomene gezeigt, wie sie im Mathematikunterricht auftreten können. Sie wurden in der $\mathrm{kWelt}$ als auch in der m-Welt genauer beschrieben. $\mathrm{h}$ Kapitel 4 wird versucht, Ursachen für die Vorstellungen aufzuzeigen.

\section{Messbarkeit von Momentangeschwindigkeit}

In Aufgabenteil c) der Untersuchung werden die Schülerinnen und Schüler u.a. gefragt, ob es eine Momentangeschwindigkeit zu einem Zeitpunkt gibt. Oft argumentieren sie wie folgt: Wenn man die Momentangeschwindigkeit messen kann, gibt es sie auch. Daher soll hier der Frage der Messbarkeit der Momentangeschwindigkeit nach- 
gegangen werden. Zunächst sollen vier Beispiele angeführt werden, wie die Momentangeschwindigkeit nicht gemessen werden kann. Diese vier Beispiele wurden mir in Gesprächen mehrmals angeboten:

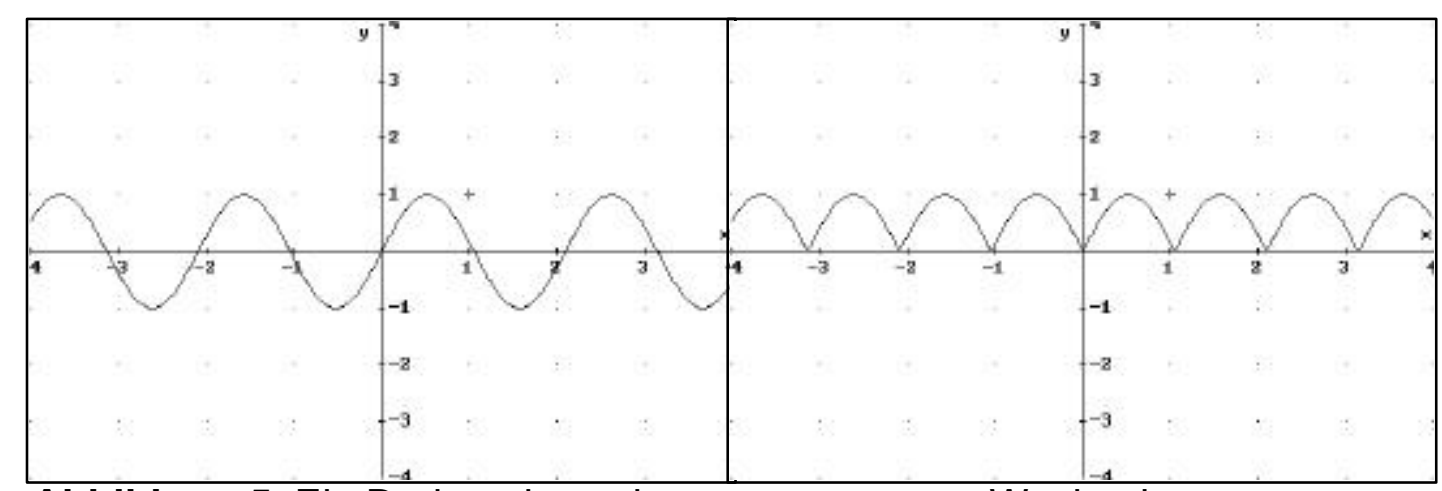

Abbildung 5: Ein Drehspulmessinstrument erzeugt Wechselstrom

Ein Tachometer misst nicht die Momentangeschwindigkeit! Bei digitalen Fahrradtachometer wechselt die Anzeige in gewissen festen Zeitintervallen. Mit Hilfe eines Magneten, der in den Speichen eines Rades befestigt ist und Impulse in einem am Rahmen montierten Leiter erzeugt, wird die Anzahl der Radumdrehungen in jedem Zeitintervall erfasst. Mittels des vorprogrammierten Radius des Rades wird der Weg berechnet und durch das Zeitintervall geteilt. Aufgrund der Zählung der vollen Radumdrehungen ist das ein diskretes Verfahren, das eben nur Durchschnittsgeschwindigkeiten misst. - Auch ein Zeigertachometer im Auto oder am Fahr rad zeigt keine Momentangeschwindigkeit an. Durch die Tachowelle wird im Gehäuse ein Permanentmagnet in einer Spule gedreht und dadurch ein Wechselstrom erzeugt. Der Zeiger des Tachos zeigt die Stromstärke an, die in einem streng monoton wachsendem Verhältnis zur Umdrehungszahl der Welle steht, die wiederum der Umdrehungszahl der Räder des Autos entspricht, also seiner Geschwindigkeit. Eigentlich müsste der Zeiger nun ständig entsprechend der sinusartig verlaufenden Ströme der Spule hin und her pendeln, siehe Abbildung 5 links. Die Größe der Amplituden wie die Frequenz (Periodenlänge) ist hier von der Umdrehungszahl, also von dem Tempo abhängig. Um das Pendeln zu vermeiden, setzt man einen Gleichrichter aus Dioden ein, der, bildlich gesprochen, die „negativen Ströme positiv macht“, siehe Abbildung 5, rechts. Man sieht aber, dass auch hier der Tachometerzeiger noch schwanken müsste. Ob aufgrund der Trägheit der Nadel oder aufgrund einer weiteren elektronischen Schaltung, auf jeden Fall zeigt die Nadel einen Wert an, der zwischen 0 und 
der Amplitudengröße liegt, eine Art Mittelwert. In einer ersten Näherung entspricht der Mittelwert der Höhe eines Rechtecks mit gleichem Flächeninhalt, wie die Kurve in Abbildung 5, rechts zwischen zwei Nullstellen beschreibt. (Da sich aber die Frequenz und die Amplitudenhöhe mit wechselnder Geschwindigkeit ständig ändern, kann das nur eine Näherung sein.) Jedenfalls wird für die Ermittlung der Position der Tachonadel die Zeitdauer der halben Periode der ursprünglichen sinusartigen Kurve benötigt.

Beim digitalen Fahrradtacho wird zu einer festen Zeitdauer die Anzahl der Radumdrehungen ermittelt, beim Zeigertacho wird zu einer festen Ortsänderung (= eine halbe Radumdrehung $=$ eine halbe Spulenumdrehung) die benötigte Zeit (= Periodenlänge) ermittelt. Beides führt zu einer Diskretisierung der Messdaten.

Auch ein „Blitzgerät“, wie es die Polizei zur Tempokontrolle einsetzt, gibt nicht die Momentangeschwindigkeit wieder. Auf dem Foto kann man zwei Dinge erkennen: Die „Schlieren“ des Fahrzeugs aufgrund seines Tempos (bei Urlaubsfotos würde man sagen, sie seien verwackelt) und die weißen dünnen Markierungen auf der Fahrbahn (die natürlich nicht verwackelt sind). Anhand der Länge der Schlieren und den Abständen der Markierungen kann man ermitteln, wie weit sich das Auto in der Verschlusszeit der Kamera bewegt hat. Der Quotient ergibt die Durchschnittsgeschwindigkeit.

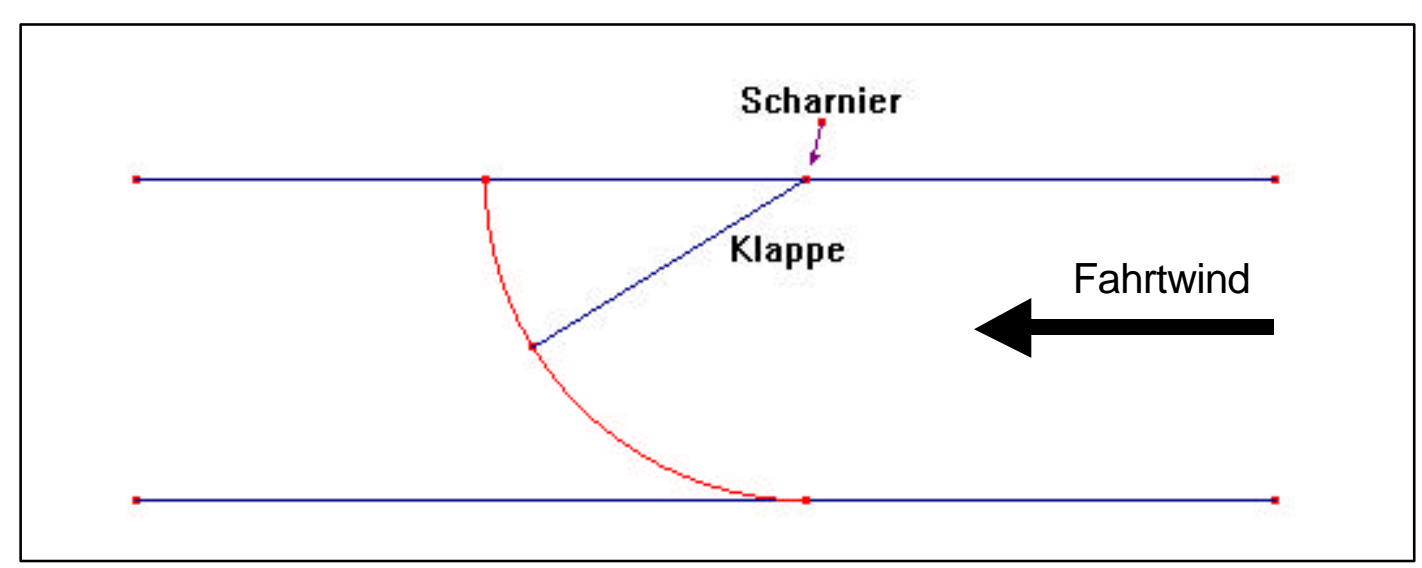

Abbildung 6: Der Röhrentacho 
Ebenso ermittelt die Radarpistole nur eine Durchschnittsgeschwindigkeit. Sie arbeitet nach dem Dopplerprinzip. Hier ist es also notwendig, mindestens eine volle Periode der reflektierten Radarwelle wieder zu empfangen, um an der Differenz der Ausgangsfrequenz und der reflektierten Frequenz die (Durchschnitts-) Geschwindigkeit des Fahrzeugs zu berechnen.

Es gibt aber ein analoges Messinstrument, mit dessen Hilfe sich im Prinzip die Momentangeschwindigkeit messen lässt, auf das mich Lutz Führer (Universität Frankfurt) in einer Diskussion aufmerksam machte, und den ich „Rohrtacho“ nennen will: Man stelle sich ein waagerecht liegendes Rohr rechteckigen Querschnitts vor. An der "Decke" befindet sich ein Scharnier an dem eine Klappe befestigt ist (siehe Abbildung 6 mit einen Längsschnitt durch das Rohr). Hängt die Klappe lotrecht herunter, verschließt sie das Rohr vollständig. Durch dieses Rohr bläst der Fahrtwind. In Abhängigkeit von der Stärke des Fahrtwinds wird nun die Klappe verschieden weit geöffnet. Der Anstellwinkel oder die Bogenlänge von der Fußstrecke der Klappe bis zur augenblicklichen Position sind Maße für die Momentangeschwindigkeit. Diese Art von Messgerät wird in Segelflugzeugen eingesetzt. Hier kann man von einem kontinuierlichen Fahrtwind ausgehen. Allerdings zeigt dieses Messinstrument die Geschwindigkeit des Segelflugzeugs bzgl. der Luftströmungen außerhalb des Fliegers an. Interessant ist, dass beim Röhrentacho weder Ortsänderungen noch Zeitspannen eine Rolle spielen.

Den Fahrtwind, der für den Öffnungswinkel der Klappe verantwortlich ist, kann man auch sinnlich wahrnehmen, wenn man z.B. bei einer Autofahrt die Hand aus dem Fenster hält. Da die Durchschnittsgeschwindigkeit über einem Zeitraum gebildet wird, kann man sie nicht sinnlich wahrnehmen. Vielleicht kann man sich aber diesen Zeitraum ins Gedächtnis rufen, sich das in diesem Zeitraum Wahrgenommene als Ganzes zugleich präsent machen. Ginge das, könnte man sich zumindest die Durchschnittsgeschwindigkeit denken, sie (nur) mittelbar wahrnehmen. Als sinnliche Wahrnehmung wie den Luftdruck auf der Hand kann das aber nicht mehr bezeichnet werden.

Das Prinzip des Röhrentachos lässt sich auch für Messungen von Momentangeschwindigkeit bewegter Körper nutzen: Prallt eine bewegte Kugel auf die Klappe, 
wird diese in Abhängigkeit der Aufprallgeschwindigkeit, das ist eine Momentangeschwindigkeit, entsprechend weit geöffnet. So könnte Newtons „letzte Geschwindigkeit“" gemessen werden, vgl. 1.2.

\section{Gibt es Momentangeschwindigkeit? - Entscheidungskriterien der Schülerinnen und Schüler}

Im Aufgabenteil c) werden die Schülerinnen und Schüler gefragt, ob es so etwas wie eine Momentangeschwindigkeit gibt. Die Transkripte lassen mindestens vier verschiedene Grundlagen erkennen, auf denen die Schülerinnen und Schüler ihre Entscheidung treffen:

\section{Vorstellung eines Bewegungsvorgangs in der r-Welt:}

Oft wird die Frage aufgrund der Vorstellung eines Bewegungsvorgangs entschieden. Dabei können die Schülerinnen und Schüler zu unterschiedlichen Ergebnissen kommen: „Sicher hat der auch ne Momentangeschwindigkeit. Weil, der ist ja zwischendurch nicht angehalten.“99; bzw.: Zu einem Zeitpunkt wird kein Weg zurückgelegt, also gibt es keine Momentangeschwindigkeit. ${ }^{100}$; bzw. Bewegungen finden nur in Zeiträumen statt, Aussagen über Momentangeschwindigkeiten sind nicht möglich. Diese Entscheidungsgrundlage kann man der r-Welt zuschreiben.

\section{Messbarkeit:}

Die Messbarkeit der Momentangeschwindigkeit bildet eine weitere Entscheidungsgrundlage. Auch sie kann der r-Welt zugeordnet werden, oder, wenn man Messvorgänge der Physik zuschreibt, auch einer Physik-Welt. Auch hier kommen verschiedene Schülerinnen und Schüler zu verschiedenen Ergebnissen: „Doch, das muss es geben. Wenn das ja, wenn er um 16 Uhr fährt, muss da auf seinem Tacho irgend etwas stehen, wie viel Kilometer er fährt.“"101 bzw. „Wie gesagt, er muss ja um 16 Uhr, ... irgend ne Geschwindigkeit gehabt haben ... Das heißt nein. Geschwindigkeit kann man nur messen, wenn man einen Zeitraum hat. “102

\footnotetext{
${ }^{99}[01 ; 33-34]$

${ }_{100}^{10}$ vgl. z.B. [13; $\left.245-253\right]$ und $[14 ; 157-158]$

$101[02 ; 64-65]$

$102[08 ; 243-245]$
} 


\section{Berechenbarkeit:}

Oft, wenn versucht wird, den Aufgabenteil c) mit Hilfe der Berechnung der Momentangeschwindigkeit zu beantworten, wird die Berechenbarkeit der Momentangeschwindigkeit als Entscheidungsgrundlage gewählt ${ }^{103}$. Kann die Momentangeschwindigkeit nicht berechnet werden, folgt aber für die Schülerinnen und Schüler nicht automatisch, dass es die Momentangeschwindigkeit nicht gibt: „Es gibt eine Momentangeschwindigkeit, wir können sie jedoch nicht ermitteln, da nicht genügend Informationen gegeben sind.“"104 Mit Informationen sind Funktionswerte der Weg-ZeitFunktion gemeint ${ }^{105}$. Einige Schülerinnen und Schüler sind aber auch der Meinung, dass man die Momentangeschwindigkeit prinzipiell nicht berechnen kann: „S: Ja hier steht doch mit deiner Methode. A: Ja, vielleicht gibt's ja mehrere, aber du kannst es mit keiner richtig machen. " ${ }^{106}$ Weiterhin gibt es Schülerinnen und Schüler, die nur ihr eigenes mathematisches Vermögen anzweifeln: „Es gibt eine Momentangeschwindigkeit, aber wir können sie nicht ermitteln.“ ${ }^{107}$

\section{Interpretation der Aufgabenstellung:}

Einige Schülerinnen und Schüler versuchen die Frage aufgrund der Aufgabenstellung und aufgrund von Reaktionen des Versuchsleiters zu beantworten: „Beide lesen Aufgabenteil d), lachen. S: Hähä... A: Also gibt's die doch!“108 „Das, das ist,... Wenn ses dieses fragen, dann muss es eine Momentangeschwindigkeit geben.“ 109 „H: Bist Du sicher, dass es die gibt? ... Tja. VL: Deckt doch mal die nächste Teilaufgabe auf! $\mathrm{K}$ : Also gibt's die! ${ }^{1110}$

\footnotetext{
103 vgl. z.B. [01]

104 [10], schriftliche Bearbeitung zu Aufgabenteil c)

105 vgl. z.B. [10; 117]

106 [11; 163 - 165]

$107[10 ; 111-112]$

$108[13 ; 75-76]$

109 [06; $322-323]$

110 [05; $411-414]$
} 


\section{Die isolierende und die einbettende Sichtweise}

Wie im vorhergehenden Kapitel angedeutet, bereitet die Vorstellung von Momentangeschwindigkeit in der Alltags- oder realen Welt einerseits keine Probleme („Ja, muss es ja geben. Er fährt ja immer weiter.“"111), andererseits sehr wohl (Zu einem Zeitpunkt bewegt sich ein Körper nicht, hat also keine Momentangeschwindigkeit, siehe oben, Alexander). In der Mathematik-Welt kann es passieren, dass dieselben Schülerinnen und Schüler den Grenzwert des Differenzenquotienten berechnen und dennoch in ihren Vorstellungen das 0/0-Problem haben, wenn sie die Grenzwerte von Zähler und Nenner des Differenzenquotienten getrennt betrachten.

In diesem Abschnitt werden zwei Sichtweisen als Erklärungsversuch für diese Phänomene entwickelt. Die Sichtweisen sollen die „isolierende Sichtweise“ und die „einbettende Sichtweise“ genannt werden. Folgende Tabelle 2 zeigt die bereits bekannten typischen Argumentationen, den Sichtweisen und Denkwelten zugeordnet. Anschließend zeige ich, dass die Sichtweisen das Grundparadoxon des infinitesimalen Denkens beschreiben und daher die lokale Änderungsrate als Grundvorstellung dieses Grund paradoxon vollständig enthält.

\begin{tabular}{|c|c|c|c|}
\hline & \multicolumn{2}{|c|}{ Denkwelten } \\
\hline & & $r$-Welt & m-Welt \\
\hline \multirow{2}{*}{ 兽 } & $\begin{array}{l}\text { isolierende } \\
\text { Sichtweise }\end{array}$ & $\begin{array}{l}\text { Zu einem Zeitpunkt legt man } \\
\text { keinen Weg zurück, daher gibt } \\
\text { es keine Momentangeschwin- } \\
\text { digkeit. - Bewegung findet nur } \\
\text { in einem Zeitraum statt. } \\
\end{array}$ & $\begin{array}{l}\text { Die lokalen Änderungen von } \\
\text { Weg und Zeit sind Null, daher } \\
\text { ist die lokale Änderungsrate } \\
\text { auch Null, bzw. 0/0 -Problem }\end{array}$ \\
\hline & $\begin{array}{l}\text { einbettende } \\
\text { Sichtweise }\end{array}$ & $\begin{array}{l}\text { Wenn man sich bewegt, hat } \\
\text { man zu jedem Zeitpunkt der } \\
\text { Bewegung eine positive } \\
\text { Momentangeschwindigkeit. }\end{array}$ & $\begin{array}{l}\text { Die lokale Änderungsrate ist } \\
\text { der Grenzwert des } \\
\text { Verhältnisses } \frac{\Delta f}{\Delta t}\end{array}$ \\
\hline
\end{tabular}

Tabelle 2: Typische Argumentationen, den Sichtweisen und Denkwelten zugeordnet

${ }^{111}$ [03; 273 - 275] 


\subsection{Die Sichtweisen in der $r$-Welt}

Bei einer Bewegung eines Körpers über einen Zeitraum ohne (vorübergehenden) Stillstand, kann man annehmen, dass er zu jedem Zeitpunkt der Bewegung eine positive Momentangeschwindigkeit hat. Wie die Transkripte zeigen, ist diese Schlussfolgerung jedoch nicht für alle Schülerinnen und Schüler zwingend. Obwohl innen wahrscheinlich klar ist, dass der von innen betrachtete Zeitpunkt innerhalb des Zeitraums der Bewegung liegt, scheint es so, als versuchten sie, sich die Bewegung nur zu einem bestimmten Zeitpunkt vorzustellen. Daraus resultiert wahrscheinlich das oben beschriebene Problem: Die Vorstellung einer Bewegung oder einer Geschwindigkeit zu einem Zeitpunkt erscheint sinnlos.

Für jeden Zeitpunkt der Bewegung kann man aber auch versuchen, die Bewegung nicht nur in diesem Moment zu sehen, sondern sie zudem als Ganzes ins Auge zu fassen: Wenn man sich die ganze Zeit bewegt, bewegt man sich auch zu jedem Zeitpunkt der Bewegung, hat also zu jedem Zeitpunkt eine Momentangeschwindigkeit. - Betrachtet man eine Bewegung über einen Zeitraum als Ganzes, bereitet es wohl keine Mühe, sich vorzustellen, dass zu jedem Zeitpunkt eine Momentangeschwindigkeit vorliegt. Bei der Schülerin Claudia hört sich das so an:

\footnotetext{
„Weil ich ja in dem Moment, die genau diese Geschwindigkeit (klopft mit einem Stift auf den Tisch und lässt die Stiftspitze auf einem Punkt), zu diesem Zeitpunkt, ich habe in diesem Moment vielleicht keine Strecke zurückgelegt, (bewegt den Stift über den Tisch) aber wenn ich das im Ganzen betrachte, würd ich, bin ich in dem Moment so schnell.“112
}

Fasst man die Bewegung zudem als Ganzes ins Auge, setzt man von vorne herein die Existenz der Momentangeschwindigkeit zu allen Zeitpunkten der Bewegung voraus. Wenn es einem also gelingt, die Bewegung als Ganzes ins Auge zu fassen, kann einem klar werden, dass es auch zu dem vorübergehend isoliert betrachteten Zeitpunkt der Bewegung eine Momentangeschwindigkeit geben muss. Denn die Bewegung findet von „vor bis nach dem Zeitpunkt“ statt, also auch an dem Zeitpunkt. Dieser wird nun in der Menge der Zeitpunkte des Zeitraums eingebettet gesehen. Ebenso kann auch die zeitweilig problematisch erscheinende, weil isoliert betrachtete

112 [14; 168 - 172] 
Momentangeschwindigkeit in die der Menge aller Momentangeschwindigkeiten über den gesamten Zeitraum eingebettet gesehen werden. Es ist also eine Frage der Sichtweise, ob man einem bewegten Körper zu einem Zeitpunkt eine Momentangeschwindigkeit zuschreibt, oder nicht.

Für die gedankliche Einbettung einer vormals isoliert betrachteten Momentangeschwindigkeit ist die Alltagsvorstellung, dass Geschwindigkeitsänderungen nicht sprung haft stattfinden, von Bedeutung. Denn dann kann man in der m-Welt von einer stetigen Geschwindigkeit-Zeit-Funk tion ausgehen. Die „benachbarten“ Funktionswerte bilden nun eine Art Referenzmenge für die betrachtete Momentangeschwindigkeit, die in dieser Wertemenge integriert ist. Sie bilden eine Art Rahmen für die speziell betrachtete Momentangeschwindigkeit und lassen aufgrund der r-Welt-Situation keine sprung hafte Veränderung zu.

Die Beschreibung der Sichtweise, bei der man die jeweiligen Größen (Zeitpunkt und Momentangeschwindigkeit) in ihren Referenzmengen eingebettet sieht, kann die Vermutung nahe legen, dass diese Größen zunächst gar nicht eingebettet sind. Das ist aus mathematisch-physikalischer Sicht natürlich falsch. Aber das EingebettetSehen oder das "Einbetten" (wenn man zuerst isoliert betrachtet hat) ist eine Aktivität des denkenden Subjekts. In der r-Welt bedeutet das folgendes: Sobald man die Bewegung als Ganzes sieht, geht man davon aus, dass sie sich stetig (im Altagssinn) verhält und dass zu jedem Zeitpunkt der Bewegung eine positive Momentangeschwindigkeit vorliegt. Also sind alle Momentangeschwindigkeiten (also auch die speziell betrachtete) von vorne herein vorhanden. Jede speziell betrachtete Momentangeschwindigkeit ist in die anderen eingebettet, sie betten sich in diesem Sinne gegenseitig ein. Sie werden aber nicht immer so gesehen. Einige Schülerinnen und Schüler isolieren nämlich sowohl den Zeitpunkt als auch die zugehörige Momentangeschwindigkeit von ihren jeweiligen Referenzmengen. Einen bewegten Körper zu einem Zeitpunkt isoliert zu betrachten bedeutet, gedanklich den Rest der Bewegung (wieder) auszublenden. Einer so isoliert betrachteten Momentangeschwindigkeit fehlt die Referenzmenge. Beim Sichtweisenwechsel zur einbettenden Sichtweise muss der Rest der Bewegung wieder in den Blick genommen werden und der spezielle Zeitpunkt mit der zugehörigen Momentangeschwindigkeit in diesem Sinne erst noch hinzugefügt und eingebettet werden. Natürlich kann es aufgrund eines außermathe- 
matischen Kontextes sinnvoll oder gar notwendig sein, gewisse Situationen lokal zu betrachten. Lokal bedeutet aber eben nicht isolierend hinzuschauen. Einbettend bedeutet eben auch nicht, die Situation ausschließlich als Ganzes zu betrachten. Um eine lokale Eigenschaft wie Momentangeschwindigkeit mittels der einbettenden Sichtweise begreifen zu können, muss man sowohl den Zeitpunkt als auch die Bewegung als Ganzes im Blick behalten.

In der m-Welt, der formalen Sprache Mathematik, ist mit einer differenzierbaren Funktion auch die Ableitungsfunktion gegeben. Somit ist mit der gegebenen Funktion bereits die Menge der Werte der Momentangeschwindigkeiten und auch die Geschwindigkeit zu dem besagten Zeitpunkt vorhanden. In dem Sinne muss keine Einbettung mehr stattfinden, es muss nur „mit der richtigen Sicht“ hingeschaut werden.

Sichtweisen müssen eingenommen werden. Da dies eine Aktivität des denkenden Subjekts ist, sollen sie ,einbettende Sichtweise" bzw. ,isolierende Sichtweise" und nicht "eingebettete" bzw. "isolierte Sichtwiese" genannt werden. Schließlich werden auch nicht die Sichtweisen eingebettet oder isoliert, sondern Größen, hier Zeitpunkte und Momentangeschwindigkeiten.

Das bedeutet: Liegt als Beschreibung eines Bewegungsvorgangs "nur“ eine WegZeit-Funktion vor und soll eine Momentangeschwindigkeit bestimmen werden, muss man einen Zeitpunkt der Bewegung in den Blick nehmen, ohne die Bewegung als Ganzes aus den Augen zu verlieren. Meine These ist, dass die einbettende Sichtweise wesentlich dazu beiträgt, die Existenz der Momentangeschwindigkeit in der rWelt plausibel erscheinen zu lassen.

Über den Wert der erwarteten Momentangeschwindigkeit wird hier noch nichts gesagt, da man die Geschwindigkeit-Zeit-Funktion auch nicht näher kennt, sondern ihr Vorhandensein nur intuitiv voraussetzt und gedanklich ausnutzt. Man erwartet jedoch, dass es eine Zahl gibt, die ihren Wert angibt. Diese müsste man ja z.B. an einem Tacho ablesen können ${ }^{113}$. Es bleibt also die Frage, welche Vorstellungen in der m-Welt geeignet sein könnten, damit der übliche Ableitungskalkül sinnhaft für die

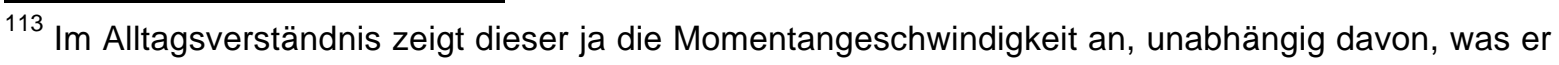
tatsächlich anzeigt, vgl. 3.2.
} 
Ermittlung des Werts der Momentangeschwindigkeit aus der Weg-Zeit-Funktion erscheint.

\subsection{Einbettung zur Ermittlung des Grenzwerts}

Was bedeutet die einbettende Sichtweise in der r-Welt für die m-Welt? Was passiert, wenn man „nur" eine Weg-Zeit-Funktion als Informationsquelle über eine Bewegung zur Verfügung hat und speziell die Frage nach der Geschwindigkeit zu einem Zeitpunkt und nach deren Wert stellt? In der Alltagsvorstellung ist klar, dass es eine Geschwindigkeit-Zeit-Funktion geben muss. Sie könnte z.B. durch einen Fahrtenschreiber, wie im einem LKW gebräuchlich, aufgezeichnet werden. Man erwartet in der $\mathrm{F}$ Welt also die Existenz eines Werts, der die Momentangeschwindigkeit angibt. Dieser Wert soll in der m-Welt ermittelt werden, eine "gute“ Mathematik sollte die Berechnung ermöglichen.

Der zur einbettenden Sichtweise in der FWelt adäquate Kalkül sollte natürlich der Grenzprozess am Differenzenquotienten $d(t)=\frac{f(t)-f\left(t_{0}\right)}{t-t_{0}}$ der Weg-Zeit-Funktion $f$ sein: Mit der Schreibweise $\lim _{t \rightarrow t_{0}} \frac{f(t)-f\left(t_{0}\right)}{t-t_{0}}$ wird der gesuchte Wert beschrieben. Warum? Und: Welcher Wert ist gemeint? Und vor allem: Gibt es in der m-Welt eine einbettende und eine isolierende Sichtweise, die, ähnlich wie in der r-Welt, adäquate und ungeeignete Vorstellungen von dem Kalkül nahe legen?

Mit dem m-Welt-Objekt "Differenzenquotientenfunktion an der Stelle b" kann man Durchschnittsgeschwindigkeiten bzgl. des Zeitpunkts $\hbar$ berechnen. Man kann nun eine Umgebung um $t_{0}$ betrachten, indem man gedanklich mit $t$ in der Umgebung über $t_{0}$ hin- und herwandert und dabei die Werte des Differenzenquotienten beobachtet. Die Differenzenquotientenfunktion an der Stelle $t_{0}$ hat bei $t_{0}$ eine stetig hebbare Definitionslücke. In der m-Welt kann man solche hebbaren Lücken bzgl. der Definitionsmenge (hier Zeit) und der Wertemenge (hier Durchschnittsgeschwindigkeiten) als eingebettet betrachten. Bedenkt man die r-Welt-Bedeutung der Differenzenquotientenfunktionswerte bei $t_{0}$, wenn man z.B. „von links mit $t$ an $t_{0}$ heranwandert“, stellt man fest, dass sich die Durchschnittsgeschwindigkeitswerte dem erwarteten Momentangeschwindigkeitswert (den man nicht kennt aber für existent hält) immer weiter 
nähern, in diesem Sinne genauer werden. „Geht man über $t_{0}$ hinaus“, werden die Werte wieder ungenauer. Die Intuition der Einbettung (in der r-Welt) fordert die Existenz eines Werts der Momentangeschwindigkeit bei $t_{0}$, und die eben aufgeführten Betrachtungen legen nahe, dass der fehlende Lückenwert der Differenzenquotientenfunktion (in der m-Welt) genau dieser Wert ist. Die Einbettung bezieht sich nicht wie bei den reinen $r$-Welt-Überlegungen nur auf die Wertemenge der intuitiv vorhandenen Geschwindigkeit-Zeit-Funktion. In der m-Welt kann der Grenzwert (bzw. die Definitionslücke) zudem als eindeutiger Häufungspunkt der Wertemenge der Differenzenquotientenfunktion bzgl. der Umgebung um to eingebettet gesehen werden. Man kann also von einer doppelten Einbettung sprechen, vgl. Abbildung 7. Die Interpretation des Grenzprozesses in der r-Welt sichert die Sinnhaftigkeit des mathe matischen Tuns.

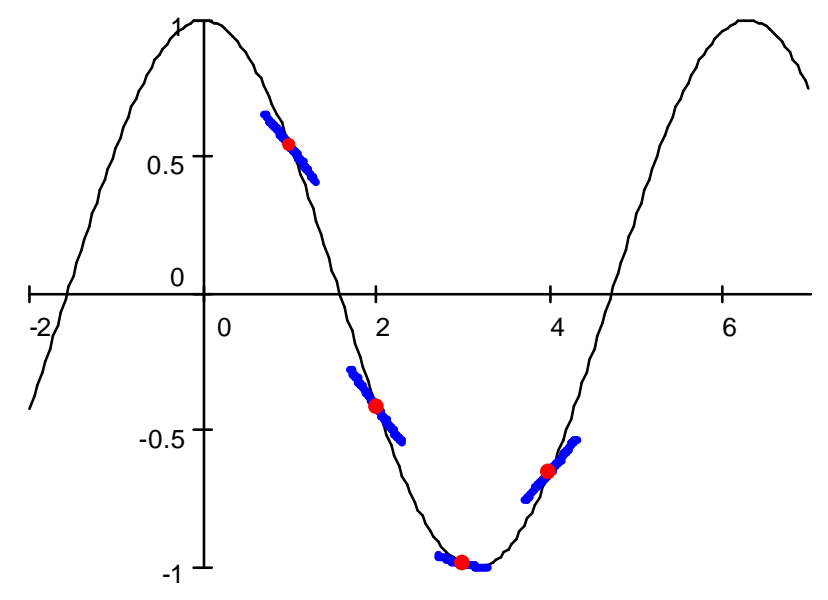

Die Abbildung zeigt den Graphen einer Geschwindigkeit-Zeit-Funktion und Abschnitte von Durchschnittsgeschwindigkeitsfunktionen an den Stellen 1, 2, 3 und 4. Die Schnittpunkte der Graphenabschnitte mit der Geschwindigkeit-Zeit-Funktion sind die Lücken. Da sich der Graph und die Graphenabschnitte schneiden, kann man erkennen, wie sich die Werte der Durchschnittsgeschwindigkeitsfunktionen dem Wert der jeweiligen Momentangeschwindigkeit (= Lückenwert) nähern, wenn bzgl. der jeweiligen Lückenstelle immer näherliegende Stellen betrachtet werden. Der Lückenwert ist sowohl in die Geschwindigkeit-Zeit-Funktion als auch in die Durchschnittsgeschwindigkeitsfunktion eingebettet.

Abbildung 7: Die doppelte Einbettung von Lückenwerten 
Die Idee, den Grenzwert der Differenzenquotienten als Lückenwert der zugehörigen Differenzenquotientenfunktion zu betrachten, ist nicht neu. Sie wird gelegentlich als eine Variante der Definition der Differenzierbarkeit angeführt:

„Definition Die reelle Funktion $\mathrm{f}$ sei auf dem (völlig beliebigen) Intervall I definiert.

Wir sagen, f sei differenzierbar im Punkte $\xi \in \mathrm{I}$, wenn [...] die Funktion

$$
F_{\xi}(x):=\left\{\begin{array}{c}
\frac{f(x)-f(\xi)}{x-\xi} \\
f^{\prime}(\xi)
\end{array} \text { in } \xi \text { stetig ist." }{ }^{114}\right.
$$

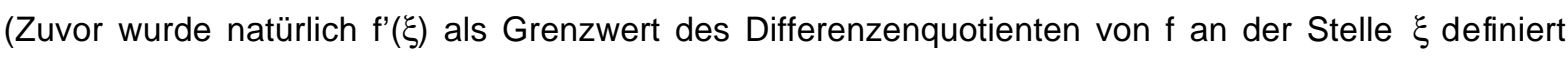
und die Existenz des Grenzwerts voraus gesetzt.)

Die zur Einbettung in der m-Welt benötigten Objekte kann man (natürlich) den Schreibweisen der heute gebräuchlichen Kalküle ansehen: Bei der $\varepsilon$ - $\delta$-Definition des Grenzwerts des Differenzenquotienten stellen die gleichnamigen Umgebungen die oben genannten Referenzmengen dar. Beim lim-Kalkül bedeutet „t $\rightarrow t_{0}$ " bei der Schreibweise $\lim _{\mathrm{t} \rightarrow t_{0}}$ den All-Quantor „für alle Folgen mit Grenzwert to“. Betrachtet man nun alle Folgenwerte aller Folgen zugleich, dann kann man diese Menge wieder als eine Art $\delta$-Umgebung auffassen und somit auch die $\varepsilon$-Umgebung erhalten.

Auch in der deutschsprachigen Mathematikdidaktik findet sich z.B. bei Griesel die Idee des Lückenwerts bei der Differenzenquotientenfunktion. Ausführliche Vorüberlegungen und Veranschaulichungen (in der g-Welt) münden in die Definition:

Definition: Die (Ausgangs-) Funktion $f$ heißt an der Stelle a differenzierbar, falls die Differenzenquotientenfunktion von $\mathrm{f}$ an der Stelle a eine Lücke hat.

Bei dieser Definition wird der Begriff „Lücke“ naiv anschaulich gefasst. Eine Präzisierung soll später erfolgen. Der Begriff „Lückenwert“ wird ebenfalls nur anschaulich gefasst. Er stellt die Zahl dar, durch welche die Lücke „vollgestopft“ wird.

Der Lückenwert gibt die Steigung der Tangente an. Das ist kein Satz, sondern eine Definition.“115

\footnotetext{
${ }_{114}$ [Heuser 1990], S. 261

115 [Griesel 1976], S. 30
} 
Da man sich hier der Lücke der Quotientenfunktion bewusst ist, vermeidet man vielleicht das 0/0-Problem, da man ständig den Quotienten im Blick behält und nicht die Grenzwerte von Zähler und Nenner getrennt betrachtet.

Die Stetigkeit der intuitiv vorhandenen Geschwindigkeit-Zeit-Funktion erleichtert einerseits die einbettende Sichtweise, birgt aber andererseits auch die Gefahr, dass bei Schülerinnen und Schülern der Eindruck entsteht, Ableitungsfunktionen seien grund sätzlich stetig. Auf dieses Problem wird in Kapitel 4.4 eingegangen.

\subsection{Die isolierende Sichtweise}

In beiden Welten ist die einbettende Sichtweise für Schülerinnen und Schüler nicht selbstverständlich, wie die Transkripte zeigen. Durch ihre isolierende Sichtweise unterliegen sie einer Fehlvorstellung. Steht „nur“ eine Weg-Zeit-Funktion zur Verfügung, kann die Frage nach der Geschwindigkeit zu einem Zeitpunkt Probleme hervorrufen, wenn aufgrund der Formulierung „zu einem Zeitpunkt“ die Bewegung nicht mehr als Ganzes gesehen wird. Wird der Zeitpunkt nicht mehr in seinem Zeitraum eingebettet sondern isoliert gesehen, kann man der Weg-Zeit-Funktion nur noch entnehmen, wo sich der Körper zu einem Zeitpunkt befindet, bzw. welche Strecke er in dem Zeitraum zurückgelegt hat. Nicht nur der Zeitpunkt wird isoliert gesehen, sondern da nun auch die Intuition keine Geschwindigkeit-Zeit-Funktion zur Verfügung stellt (zu einem Zeitpunkt kann man sich ja nicht bewegen), kann die Momentangeschwindigkeit dort nicht eingebettet gesehen werden. In der r-Welt werden bei dieser isolierenden Sichtweise der Zeitpunkt und der zugehörige Ort isoliert betrachtet. Ist "nur" eine WegZeit-Funktion gegeben, betrachtet man in der m-Welt die Diffe renzenquotientenfunktion. Sie ist an der besagten Stelle jedoch nicht definiert. Die isolierende Sichtweise führt aber dazu, nur diese nicht definierte Stelle zu betrachten. Man kann also keine Aussagen über irgendwelche Eigenschaften dieser Funktion treffen.

Aber nicht nur der Zeitpunkt wird isoliert betrachtet, sondern auch der Zeitraum, wenn Schülerinnen und Schüler sagen, dass Bewegungen und somit Geschwindigkeiten nur über Zeiträume stattfinden. Dann werden die Zeitpunkte des Zeitraums 
ausgeblendet, da für diese ja keine Aussagen getroffen werden können. „Isolierende Sichtweise" heißt also, dass Zeitpunkt und Zeitraum voneinander isoliert betrachtet werden. Beide Isolationsvorgänge hängen eng zusammen, da sich die charakteristischen Argumentationen und die Schlussfolgerungen daraus entsprechen: Da zu einem Zeitpunkt keine Bewegung stattfindet, gibt es keine Momentangeschwindigkeit; bzw.: Da Bewegung nur in einem Zeitraum stattfindet, gibt es nur Durchschnittsgeschwindigkeit. Letztlich liegt also nur ein Isolationsvorgang vor.

Wird die Weg-Zeit-Funktion als einzige Informationsquelle über die Bewegung genutzt, muss man auf die Orts-Änderungs-Rate zurückgreifen und den zugehörigen Grenzprozess betrachten. Da es nach der isolierenden Sichtweise keine Momentangeschwindigkeit gibt, messen Schülerinnen und Schüler, die dennoch den zugehörigen Wert des Diffe renzialquotienten berechneten, diesem Wert keinerlei außermathematische Bedeutung zu oder sie weichen gedanklich dem Zeitpunkt wieder aus und betrachten unendlich kleine Zeitintervalle: „Also, wir haben jetzt, hier ist es nicht gleich Null, sondern es geht gegen Null, unendlich klein.“ 116

Eine Verstärkung der Fehlvorstellung findet dann statt, wenn die lokalen Änderungsraten der Zähler- und Nennerfunktion des Differenzenquotienten getrennt betrachtet werden. Die Vorstellung einer Bewegung und einer Momentangeschwindigkeit als lokale Änderungsrate erscheinen sinnlos, da nun Orts- und Zeitänderung beide Null sind. Das 0/0-Problem drängt sich auf. Weil man zu einem Zeitpunkt keinen Weg zurücklegt, kann man auch keine Momentangeschwindigkeit haben. Somit können die Referenzmengen, in denen die Momentangeschwindigkeit und ihr Wert eingebettet gesehen werden können, verloren gehen.

Das im Unterricht oft gewählte Sekanten-Tangenten-Problem ist „nur" eine Einkleidung der lokale Änderungsrate und schützt vor der isolierenden Sichtweise nicht: Ein Punkt kann keine Steigung haben und schon gar nicht eine eindeutig bestimmte. Erst als „Mitglied einer Kurve“ (und hier geschieht die Einbettung!!) kann man der Kurve in dem Punkt (nicht dem Punkt selber!!) die Eigenschaft „Steigung“ zuschreiben.

${ }^{116}[14 ; 218-218]$ 
Fazit: An den Überlegungen wurde deutlich, dass Differenzierbarkeit an einer Stelle eine lokale, aber keine punktuelle Eigenschaft ist. Zur Berechnung der lokalen Änderungsrate an nur einer Stelle müssen die Funktionswerte in einer ganzen Umgebung um die Stelle herum bekannt sein. Die lokale Eigenschaft „lokale Änderungsrate“ ist aus der alleinigen Kenntnis der punktuellen Eigenschaft „Funktionswert an der Stelle“ nicht zu ermitteln, die zugehörige Differenzenquotientenfunktion hat dort eine Definitionslücke. Und obwohl die (stetige) Funktion an einer Stelle keine lokale Änderung hat, kann sie an derselben Stelle eine positive lokale Änderungsrate haben.

\subsection{Zwei Bemerkungen zu den Sichtweisen}

\section{Prototypische Argumentationen}

Die Ausschärfung der beiden Sichtweisen war bei mir nicht von Anfang an vorhanden, sondern wurde durch die intensive Beobachtung der Arbeitsphasen der Schülerinnen und Schüler anhand der Transkripte erheblich gefördert. Die Beiträge von Claudia und Alexander gaben mir den ersten Anstoß, die Überlegungen anzustellen, aus denen schließlich die Sichtweisen hervorgingen. Aus jetziger Sicht, also mit der Kenntnis der Sichtweisen, lesen sich die Äußerungen wie Prototypen für die Auswirkungen der Sichtweisen auf das Bewegungsproblem. Alexander und Claudia haben zwar nicht gemeinsam an der Aufgabe gearbeitet, dennoch liest sich Claudias Beitrag (einbettende Sichtweise) wie eine Antwort auf Alexanders Stellung nahme (isolierende Sichtweise).

\section{Alexander:}

„Genau, weil in dem Augenblick keine Zeit vergeht. So. Und äh, theoretisch bewegt sich das Auto zu diesem Zeitpunkt nicht vorwärts. Sondern genau zu dieser Zeit bleibt es ja genau da stehen, wo ich hin gucke. [...] Ne Sekunde später ist das Auto woanders. Und dann kann ich den Weg, dann habe ich einmal den Weg, den Weg, den es zurückgelegt hat und die Zeit, und daraus ich irgendwie da die Geschwindigkeit berechnen. Also hat ne Geschwindigkeit, oder ne Beschleunigung oder wie 
auch immer, oder eine Verzögerung in diesem Zeitraum bestanden. [...] Aber genau zu dem Augenblick, wo ich kucke [...], ist es für mich gleich 0.“117

Claudia:

„Weil ich ja in dem Moment, die genau diese Geschwindigkeit (klopft mit einem Stift auf den Tisch und lässt die Stiftspitze auf einem Punkt), zu diesem Zeitpunkt, ich habe in diesem Moment vielleicht keine Strecke zurückgelegt, (bewegt den Stift über den Tisch) aber wenn ich das im Ganzen betrachte, würd ich, bin ich in dem Moment so schnell." ${ }^{118}$

Die hier aufgezeigten Zusammenhänge zwischen $\mathrm{F}$ und $\mathrm{m}$-Welt, zwischen Momentangeschwindigkeit und Grenzwert am Differenzenquotienten sind die, die Newton wahrscheinlich dazu gebracht haben, die Differenzialrechnung so zu entwickeln, wie er es getan hat. Es wird eine Mathematik entwickelt, in die man bei der Modellbildung etwas bezüglich der r-Welt Sinnvolles hineinsehen kann: Was da getan wird, ist sinnvoll (da es eine Momentangeschwindigkeit / lokale Änderungsrate gibt), wie es getan wird, ist sinnvoll (der Grenzprozess bringt immer genauere Durchschnittsgeschwindigkeiten / Änderungsraten im oben genannten Sinne) und die Bedeutung des Ergebnisses des Kalküls ist sinnvoll (das ist der Wert der Momentangeschwindigkeit / der lokale Änderungsrate) und auch die Güte (der Grenzwert ist ganz genau und nicht nur beliebig genau). Auch auf das Phänomen, dass Zähler und Nenner des Differenzenquotienten getrennt betrachtet werden, ist er in seinen Ausführungen über letzte Größen und letzte Verhältnisse eingegangen. Auch wenn weder Newton geschweige denn die Schülerinnen und Schüler in dieser Untersuchung eine formal ausgereifte Mathematik entwickeln, bietet die Aufgabe eine gute Möglichkeit, mathematisch im Sinne Freudenthals zu arbeiten, vgl. 1.1: Freudenthal fordert, aus Anwendungen Mathematik entstehen zu lassen. In diesem Sinne ist der Vorschlag der Vorstellung des Hin- und Herwanderns gemeint: Nachdem die einbettende Sichtweise eine plausible Vorstellung von Momentangeschwindigkeit in der r-Welt sicherte, wurde von Newton eine Mathematik entwickelt, die eine mathematische Beschreibung des Bewegungsproblems unter Berücksichtigung der einbettenden Sichtweise darstellt. Im Sinne Freudenthals können auch Schülerinnen und Schüler Newtons intuitiven Grenzwertbegriff nachentdecken. 


\section{Eine Gefahr der einbettenden Sichtweise}

Wenn man sich nicht gerade vorstellt, dass der Radfahrer in der Aufgabe gegen eine Wand fährt, kann man von einer stetigen Geschwindigkeit-Zeit-Funktion ausgehen. Da die Erarbeitung des intuitiven Grenzwertbegriffs an Zusammenhängen zwischen r- und m-Welt ansetzt, besteht die Gefahr, dass Schülerinnen und Schüler den Eindruck gewinnen, Ableitungsfunktionen seien grundsätzlich stetig. Der Nachweis der Tatsache, dass Ableitungsfunktionen keine Sprungstelle haben können, wird Gaston Darboux (1842 - 1917) zugesprochen. (Idee des Widerspruchsbeweises, „graphisch“ gesprochen: Hätte die Ableitungsfunktion f' der differenzierbaren Funktion $f$ eine Sprungstelle, so hätte f selber dort einen „Knick“ haben müssen, wäre dort also nicht differenzierbar gewesen.) Es kommt also nur die zweite Art von Unstetigkeit in Frage, „beliebig starkes Oszillieren“. Ein bekanntes Beispiel für eine differenzierbare Funktion, deren Ableitungsfunktion nicht stetig ist, ist:

$$
f(x)=\left\{\begin{array}{ll}
x^{2} \sin \left(\frac{1}{x}\right), & \text { falls } x \neq 0 \\
0 & , \text { falls } x=0
\end{array}, \text { vgl. Abbildung } 8\right.
$$

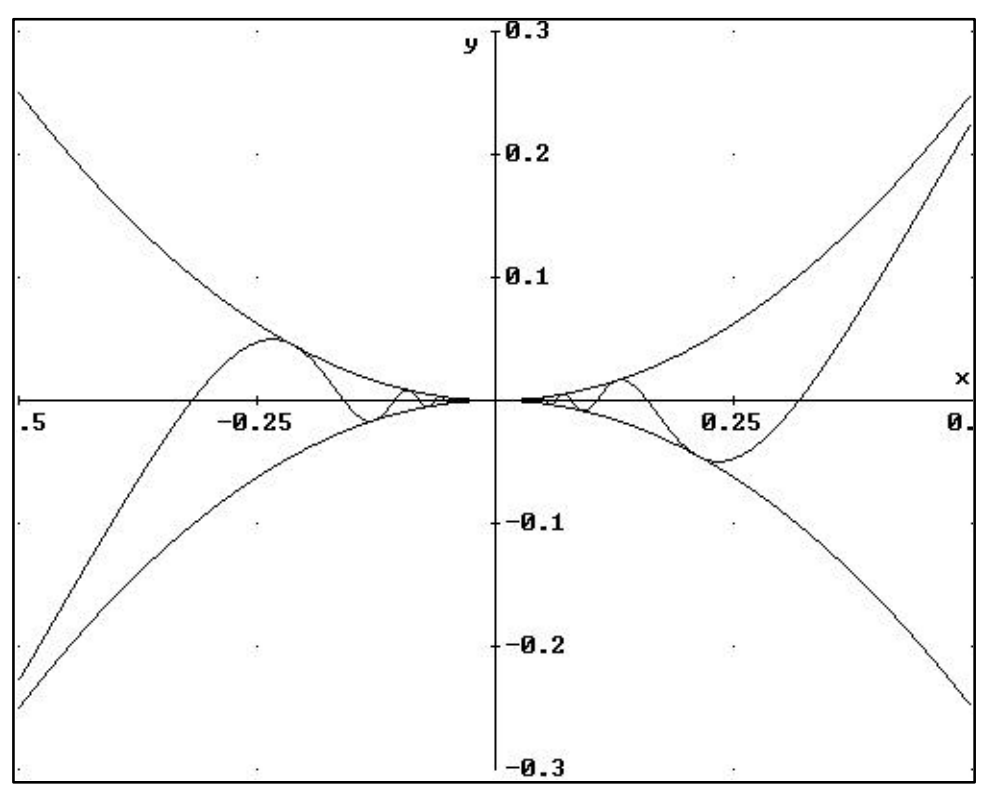

Abbildung 8: Der Graph von $\mathrm{f}$, eingeschlossen in eine „Parabelschere“ aus $x \mapsto x^{2}$ und $x \mapsto-x^{2}$ 
Für $\mathrm{x} \neq 0$ ist $\mathrm{f}$ natürlich differenzierbar, aber auch für $\mathrm{x}=0$, und zwar ist:

$$
\lim _{h \rightarrow 0} \frac{h^{2} \sin \left(\frac{1}{h}\right)-0}{h}=\lim _{h \rightarrow 0}\left(h \cdot \sin \left(\frac{1}{h}\right)\right)=0 .
$$

Somit gilt: $\quad f^{\prime}(x)= \begin{cases}2 x \cdot \sin \left(\frac{1}{x}\right)-\cos \left(\frac{1}{x}\right), & \text { falls } x \neq 0 \\ 0 & \text {, falls } x=0\end{cases}$

Man sieht, dass f' in 0 nicht stetig ist: Zwar „verschwindet“ der vordere Summand $2 x \cdot \sin \left(\frac{1}{x}\right)$ an der Stelle 0 , aber $\cos \left(\frac{1}{x}\right)$ oszilliert um diese Stelle herum beliebig stark zwischen -1 und 1.

Um den skizzierten Beweis genau führen zu können, muss neben einem intuitiven Grenzwertbegriff auch ein formaler beherrscht werden. Im empirischen Teil dieser Untersuchung steht aber der intuitive Grenzwertbegriff im Vordergrund. Das zeigt schon die Aufgabe. Sie kann meines Erachtens im Rahmen eines Einstiegs in die Differenzialrechnung eingesetzt werden. Mit ihrer Hilfe können („nur“) Vorstellungen und Intuitionen vom Grenzwertbegriff beim Ableiten gewonnen werden. Letztlich muss die Lehrperson entscheiden, in wieweit der Grenzwertbegriff im Unterricht präzisiert und formalisiert werden soll. Nach der Einführung in die Differenzialrechnung würde ich meine Schülerinnen und Schüler zudem zunächst mit Positiv-Beispielen, also mit stetig differenzierbaren Funktionen arbeiten lassen, bevor ich sie mit einem Funktionsmonster wie dem oben angeführten konfrontieren würde.

\subsection{Kritik an der lokalen Änderungsrate als Grundvorstellung}

Wenn Schülerinnen und Schüler über die Momentangeschwindigkeit diskutieren, entwickeln sie fast zwangsläufig beide Sichtweisen. Da innen plausible Argumentationen für bei zwei gegensätzliche Standpunkte zur Verfügung stehen, befinden sie sich in einem inneren Konflikt, der durch die Sichtweisen beschrieben und erläutert werden kann. Im weiteren soll dieser innere Konflikt etwas grundsätzlicher betrachtet werden. 
Kann man einen Zeitraum als aus Zeitpunkten zusammengesetzt betrachten? Folgender Schüler scheint dies zu versuchen:

„Du kannst doch schreiben, dass es keine Momentangeschwindigkeit geben würde, gibt's auch keine Durchschnittsgeschwindigkeit, weil dann wird er sich ja gar nicht bewegen. ${ }^{\text {"119 }}$

Wie in Kapitel 3.2 bemerkt, verweist diese Frage auf die viel grundsätzlichere erkenntnistheoretische Diskussion über die Topologie des Kontinuums. Denn hier zeigt sich vielleicht das Grundparadoxon des infinitesimalen Denkens (in geometrischer Fassung): Man betrachte eine Strecke. Wahr scheinlich kann man sich leichter einen Punkt der Strecke, z.B. als Schnittpunkt mit einer anderen Strecke vorstellen, also diesen Punkt als Element der gegebenen Punktmenge "Strecke“ (als Kontinuum) sehen, als dass man sich vorstellen kann, die Strecke als Kontinuum endlicher Länge (größer Null) aus Punkten ohne Ausdehnung „zusammenzusetzen“. Folgendes Schülerinnen-Zitat zeigt, wie sich das Problem des Zusammensetzens bezüglich einer Bewe gungssituation auswirken kann:

„Hör mal, wenn da, wenn da eine Momentangeschwindigkeit ist, muss er da immer ja gleich fahren. ... Oder nicht?" ${ }^{120}$

Die Schülerin scheint die Wertemenge einer Geschwindigkeit-Zeit-Funktion aus Geschwindigkeitswerten zusammengesetzt und nicht als „fertiges Kontinuum“ zu sehen: Da für sie die Geschwindigkeitswerte diskret vorliegen (isolierende Sichtweise), scheint sie sich Geschwindigkeitsänderungen nur als Sprünge vorstellen zu können. Aufgrund von r-Welt-Vorstellungen erwartet sie aber, dass die Geschwindigkeit-ZeitFunktion der Radfahr-Situation stetig ist, zwischen den Geschwindigkeitswerte also kein Sprung auftreten darf. So kommt sie zum Schluss, dass der Radfahrer „da immer gleich fahren muss". - Die Geschwindigkeitswerte scheinen für sie also diskret zu sein. Sie weiß aber sehr wohl, dass der Radfahrer seine Geschwindigkeit ändern kann. Es bleibt die Frage, was „zwischen den Werten“ passiert. Die geometrische Version der Frage (Was passiert zwischen den Punkten?) hat auch die führenden Köpfe der Zeit der mathematischen Grundlagenkrise beschäftigt. 1921 stellte

\footnotetext{
119 [03; 281 - 283]

$120[06 ; 217-218]$
} 
Weyl seine „atomistische Auffassung des Kontinuums“ vor und beantwortete die Frage z.B. so: „die kontinuierliche „Raumsauce“, welche zwischen innen ergossen ist, tritt gar nicht in die Erscheinung;" ${ }^{121}$

Auch andere bekannte Paradoxien lassen sich auf die Denkweisen „fertiges Kontinuum“ und „Zusammensetzen aus diskreten Punkten“ zurückführen. Drei Beispiele werden nun aufgeführt, zunächst das Pfeilflug-Paradoxon von Zenon:

- Ein Pfeil wird auf eine Zielscheibe geschossen. Der Pfeil wird nicht ankommen, er wird nicht mal den Bogen verlassen, denn: Man erwartet, dass der Pfeil den Mitter punkt zwischen dem Schützen und der Zielscheibe erreichen muss. Dazu muss er aber den Mittelpunkt des Mittelpunkts und dem Schützen erreichen usf. - Die Wortwahl legt die isolierende Sichtweise nahe. Dieses wiederum suggeriert, dass man anschließend die Flugbahn (Kontinuum) aus Orten (ohne Ausdehnung) zusammensetzen muss, so dass die Flugbahn die Länge Null haben muss. Dennoch: Niemand würde sich als Zielscheibe zur Verfügung stellen!!

- Das Paradoxon des unbegrenzten Teilens und Zusammensetzens von Zenon (vgl. [Becker 1975, S. 42]): Zenon beginnt mit einer Strecke endlicher Länge größer Null. Er setzt voraus und behauptet, dass, wenn man diese Strecke in zwei Teile teilt, diese Teile wiederum eine endliche Länge größer Null besitzen. Nun teilt er fortgesetzt die Teilstrecken:

\begin{abstract}
„Und von dem vor jedem liegenden Teile, d.h. dem Teil jeden Teiles, gilt dieselbe Behauptung. Auch dieser wird nämlich Größe haben, und es wird ein anderer vor inm liegen. Dieselbe Behauptung gilt nun ein für allemal. Denn kein derartiger Teil desselben (des Ganzen) wird die äußerste Grenze bilden, und nie wird der eine ohne Verhältnis zum anderen sein. Wenn so viele Dinge sind, so müssen sie notwendig zugleich klein und groß sein: so klein, daß sie keine Größe haben, so groß, daß sie unbegrenzt viele sind. “122
\end{abstract}

Und darin liegt das Paradoxon: Durch das fortgesetzte Teilen einer Strecke (man beginnt mit einem „fertigen Kontinuum“) erhält man unbegrenzt viele Teile mit Länge

\footnotetext{
121 [Weyl 1921], S. 47

122 [Becker 1975], S. 42
} 
größer Null. Setzt man aber unbegrenzt viele Teile größer Null zusammen, müssen sie zusammen unbegrenzte Länge haben. Da man aber mit einer endlichen Länge begann, müssen die Teile die Länge Null haben.

\title{
- Ein letztes Beispiel:
}

\begin{abstract}
„Wenn ein Kegel parallel der Grundfläche von einer Ebene geschnitten wird, wie soll man sich die entstehenden Schnittflächen denken, gleich oder ungleich (kongruent oder inkongruent)? Sind sie ungleich, dann werden sie den Kegel ungleichmäßig machen, da er viele stufenartige Einschnitte und Vorsprünge erhält; sind sie dagegen gleich, so werden auch die Schnitte gleich sein und der Kegel wird die Erscheinung des Zylinders darbieten, da er aus gleichen, nicht aus ungleichen Kreisen bestehen wird, was doch sehr ungereimt ist. “" ${ }^{123}$
\end{abstract}

Wieder beginnt man mit einem „fertigen Kontinuum“, dem Kegel, den man schneidet. Dann denkt man sich den Kegel aus den Schnittflächen zusammengesetzt, was zum Paradoxon führt. Die „Stufen“ und „Vorsprünge" erinnern an die Vorstellungen der Schülerin von oben: Zwischen zwei Werten einer Geschwindigkeit-Zeit-Funktion darf es ebenso wenig Sprünge geben, wie zwischen den Radien der Kreise.

Die Sichtweisen beschreiben die beiden gegensätzlichen Denkweisen des Grundparadoxons: Die einbettende Sichtweise kann man nur einnehmen, wenn man gedanklich mit einem „fertigen Kontinuum" beginnt. Dieses stellt nämlich die Referenzmenge dar, in die eingebettet werden kann. Einen Zeitpunkt kann man z.B. in das Kontinuum eines Zeitraums eingebettet sehen. Will man weiterhin einen Momentangeschwindigkeitswert einbetten, sind die Wertemengen einer Geschwindigkeit-ZeitFunktion oder einer Durchschnittsgeschwindigkeit-Zeit-Funktion mögliche Kontinua, wobei im letzten Fall die Momentangeschwindigkeit den Lückenwert darstellt, was den Einbettungsgedanken jedoch nicht behindert. - Das Zusammensetzen eines Kontinuums kann mit der isolierenden Sichtweise in Verbindung gebracht werden, wenn z.B. isoliert betrachtete Zeitpunkte zusammengefasst werden müssen, damit ein im Alltag beobachteter Bewegungsvorgang beschrieben werden kann. Dann kommt es jedoch zu den Problemen, die die oben angeführten Schülerinnen- und Schüler-Zitate andeuten. Man kann das Auftreten der Sichtweisen also als Indikator

${ }^{123}$ [Becker 1975], S. 56 
für das Auftreten des Grundparadoxons ansehen. Das Grundparadoxon ist jedoch „nur" ein erkenntnistheoretisches, wie die Maßtheorie zeigt. Diese verbindet beide Sichtweisen und zeigt letztlich, dass beide innerhalb dieser Theorie akzeptabel sind.

Die Untersuchung zeigt, dass Schülerinnen und Schüler beide Sichtweisen entwickeln, wenn sie intensiv über den Begriff der Momentangeschwindigkeit nachdenken. Da die Sichtweisen die Denkweisen des Grundparadoxons beschreiben, kann man vermuten, dass beide Sichtweisen ebenso auftreten, wenn Schülerinnen und Schüler lokale Änderungsraten in beliebigen Kontexten studieren: So ist z.B. aus dem Analysis-Unterricht bekannt, dass die Sichtweisen auch bei Überlegungen zur Steigung eines Graphen in einem Punkt auftauchen. - Die ausführlichen Transkriptanalysen in Kapitel 5 zeigen, wie Schülerinnen und Schüler durch intensives Nachdenken über die lokale Änderungsrate (in dem Fall Momentangeschwindigkeit) in einen inneren Konflikt geraten, der durch die Sichtweisen beschrieben und durch das Grundparadoxon ausgelöst und begründet wird.

Die Sichtweisen beschreiben zwei erkenntnistheoretisch zueinander paradoxe Denkweisen bezüglich der lokalen Änderungsrate. Der Begriff der lokalen Änderungsrate trägt also das Grundparadoxon bereits in sich. Der didaktische Ansatz, der die lokale Änderungsrate dem Ableitungsbegriff als Grundvorstellung unterlegt, ist daher in der gängigen Form nur bedingt brauchbar. Wie die Grundvorstellung der linearen Approximation einzuordnen ist, bleibt dahingestellt. Da aber eine Infinitesimalrechnung ohne Grenzprozesse und Grenzwerte ein Widerspruch in sich darstellt, ist zu erwarten, dass bei Vorstellungen von der linearen Approximation zu den Sichtweisen verwandte Denkweisen gefunden werden können.

Meine Untersuchung hat ergeben, dass für Schülerinnen und Schüler die einbettende Sichtweise diejenige ist, die den Zusammenhang von Prozessen in der Umwelt und lokalen Änderungsraten zur Beschreibung lokaler Eigenschaften der Prozesse in der Mathematik besonders deutlich und plausibel macht. Weiterhin wurde in Kapitel 4.2 gezeigt, wie die einbettende Sichtweise genutzt werden kann, den Grenzwert zu ermitteln. Damit ist die Frage klar, welche Sichtweise für den Analysisunterricht die wünschenswerte ist. Dass im Analysisunterricht die einbettende Sichtweise auch bedenkenlos betont werden darf, wird durch die Maßtheorie sichergestellt. Kapitel 6 
beschäftigt sich ausführlich mit dem Umgang mit beiden Sichtweisen im Analysisunterricht.

\subsection{Statische und dynamische Sichtweise bei Folgen}

In diesem Unterkapitel soll auf Peter Benders Artikel „Fehlvorstellungen und -verständnisse bei Folgen und Grenzwerten“ ${ }^{124}$ eingegangen werden. Zunächst soll gezeigt werden, dass auch Bender eine Art einbettende Sichtweise für adäquate Vorstellungen vom Grenzwert bei Folgen für notwendig hält. Er nennt sie die statische Sichtweise. Seiner statischen Sichtweise stellt er die dynamische gegenüber, die allerdings der hier entwickelten isolierenden Sichtweise nicht entspricht. Weiterhin sollen die Grenzwertbegriffe bei Folgen und am Differenzenquotienten diskutiert werden. Wenn von einer dynamischen Sichtweise oder Vorstellung die Rede ist, ist natürlich nicht gemeint, dass die Vorstellung an sich dynamisch ist, sondern dass sich jemand über dynamische Vorgänge Gedanken macht.

In seinem Artikel führt Bender Fehlvorstellungen vom Grenzwert bei Folgen unter anderem auf eine dynamische Sichtweise von Folgen zurück. Darunter versteht er das gliedweise Durchwandern einer Folge mit dem Ziel, den Grenzwert zu bestimmen. Dazu setzt man (bei explizit vorgegebenen Folgen) der Reihe nach die natürlichen Zahlen ein, um die Werte der Folgenglieder zu bestimmen. Der Wunsch, so tatsächlich an den Grenzwert gelangen zu können, führt zu Vorstellungen, es könne eine Art letztes Folgenglied geben, das den Index $\infty$ trägt, und welches man mittels des Durchwanderns auch erreichen könne. Natürlich stellt die Betrachtung eines Anfangsstücks einer Folge einen wichtigen Schritt der Analyse einer Folge dar. Durch die Betrachtung kann man zu einer Vermutung gelangen, welche Zahl der Grenzwert sein könnte. Bender schreibt das so:

„Für konvergente Folgen (besonders deutlich bei Reihen) ist charakteristisch, daß das numerisch Wesentliche am Anfang, geschieht' und die Folgenglieder sich mit wachsenden Nummern immer weniger voneinander unterscheiden. “'25

\footnotetext{
$\overline{124}$ [Bender 1991b], Hervorhebungen wie im Original

125 [Bender 1991b], S. 240, Hervorhebungen wie im Original
} 
Dann macht Bender auf die Unmöglichkeit aufmerksam, dass das Durchwandern, also der Grenzprozess zum Grenzwert führen könne: In den meisten Fällen ist der Grenzwert nicht zugleich auch Wert eines Folgenglieds. Da es aber kein letztes Folgenglied gibt, da die natürlichen Zahlen über jede Schranke wachsen, kann der Grenzwert i.A. auch nicht erreicht werden. Es liegt also am Wesen der Folge (als Funktion von den natürlichen Zahlen in die reellen), dass man weder die Definitionsmenge vollständig durchlaufen, noch in der Wertemenge den Grenzwert erreichen kann: In der Wertemenge der konvergenten Folge (in der Schulmathe matik sind das zumeist die reellen Zahlen) nähert man sich sehr wohl dem Grenzwert beliebig an, aber in der Definitionsmenge (den natürlichen Zahlen) findet keine Näherung irgendeiner Art statt, da es keine Zahl $\infty$ gibt, und selbst wenn es eine solche gäbe, was sollte dann Nähern heißen ${ }^{126}$ ?

Im 11. Absatz seines Artikels betont Bender, dass es für ein mathematisch adäquates Verständnis vom Grenzwert bei Folgen unerlässlich ist, unter der Schreibweise "lim“ (wie bei jedem algebraischen Term) zwischen der Handlungsaufforderung, den Grenzwert zu berechnen, und dem Ergebnis der Berechnung, dem Grenzwert als Zahl, zu unterscheiden. Beides wird durch diese Schreibweise repräsentiert. Die Tatsache, dass eine Schreibform zugleich Handlungsaufforderung und Ergebnis darstellt, sollte für Schülerinnen und Schüler in der Oberstufe nicht neu sein. Nur wird beim lim-Operator die Handlungsaufforderung oft missverstanden, nämlich als Aufforderung, die Folge (endlos) zu durchwandern, anstatt den Grenzwert zu berechnen. Es ist aber angemessen, den lim-Operator als Abbildung der Menge der konvergenten Folgen in die Menge der reellen Zahlen anzusehen.

Hier wird der Zusammenhang zu der zuvor entwickelten einbettenden Sichtweise sichtbar: Das Argument des lim-Operators ist eine Folge als Ganzes! Genau wie für den Begriff der Momentangeschwindigkeit die Bewegung als Ganzes betrachtet werden muss, muss auch die Folge als Ganzes betrachtet werden. Insbesondere ein

\footnotetext{
${ }^{126}$ Unter „sich Nähern“ soll hier „Abstand verringern“ verstanden werden. Der Abstand kann mittels des Betrags der Differenz von zwei Zahlen notiert werden. Daher kann z.B. der Abstand von $\infty$ und $n$ nicht betrachtet werden, da $\infty$ keine Zahl ist, somit $|\infty-n|$ eine sinnfreie Schreibform ist. Der Abstand von 0 und 1/n, also $|0-1 / \mathrm{n}|$ kann dagegen betrachtet werden: Durchwandert man gedanklich ein Anfangs stück der Folge $(0-1 / n)_{n}$, kann man beobachten, dass der Wert der Folgenglieder „Von rechts gegen Null strebt“. Hier passiert das numerisch Wesentliche, hier kann die Vermutung entstehen, dass 0 der Grenzwert der Abstands-Folge ist. Diese Beobachtung ist bei Abstands-Folgen von divergenten Folgen eben nicht möglich, da $\infty$ keine Zahl, also auch kein Grenzwert ist.
} 
Hauptteil einer Folge als Ganzes gesehen ermöglicht es erst, eine Vermutung über den Grenzwert zu verifizieren. Denn das beliebig kleine $\varepsilon$ der üblichen formalen Definition des Grenzwerts einer Folge erzwingt beliebig große Indizes der Folgenglieder, von denen an alle Folgenglieder in der $\varepsilon$-Umgebung um den vermuteten Grenzwert zu liegen haben.

Durchläuft man eine Folge in Gedanken, kann man sie nie als Ganzes sehen, und der für den Grenzwert wesentlichen Teil der Folge tritt nicht in Erscheinung, denn

„Gegen dieses numerisch Wesentliche ist das infinitesimal Wesentliche (s.o.) einer Folge deutlich abzugrenzen: Ersteres ist an Anfangsstücke, letzteres (insbesondere auch die Eigenschaft der Konvergenz selbst) an Hauptstücke der Folge gebunden. (der Ausdruck ,Hauptstück' o.ä. ist m.E. der Wendung ,Endstück' vorzuziehen, damit nicht wieder der Gedanke an ein Ende der Folge aufkommt.) ““27

Bolzano bemerkt dazu: „D.h. um mir die Vorstellung eines Ganzen aus unendlich vielen Teilen zu machen, ist es unnötig (und unmöglich), mir deren unendlich vielen Teile vorzustellen. “128 Genau das aber versucht der Denker beim Durchwandern der Folge.

Die Bezeichnung „statische Sichtweise“ für die Sicht der Folge als Ganzes taucht in Benders Artikel nur einmal auf, als Bender das Verhältnis der Sichtweisen zueinander diskutiert. Dort heißt es:

„Die Hauptthese der vorliegenden Arbeit lautet aber, daß diese sog. dynamische Auffassung von Folgen, die Schülern schon immer, also unabhängig vom Arbeitsmittel ,Computer', nahegebracht werden sollte, mit verantwortlich ist für verbreitete Fehlvorstellungen und -verständnisse (FVV) vom Begriff des Grenzwerts. Mit dieser These ist nicht die Konsequenz verbunden, die dynamische Sichtweise gänz lich zu eliminieren, die ja als Einstieg und bei Anwendungen überaus erfolgreich ist. Aber es gibt eine (nicht zeitliche, sondern epistemologische) Phase bei der Ausbildung des Grenzwertbegriffs, wo eine eher statische Betrachtungsweise geboten ist, weil die vordergründig dynamische in die Irre führt. [...] Es ist nicht ausgemacht, daß es gelingt, in den Lernprozessen der Schüler die dynamischen

\footnotetext{
$\overline{127}$ [Bender 1991b], S. 240, Hervorhebungen wie im Original

128 Bolzano: „Wissenschaftslehre“ Neudruck Leipzig 1929-31; zitiert nach Spalt, D.D. „Die Unendlichkeiten bei Bernard Bolzano“ in: [König 1990]
} 
Vorstellungen in den entscheidenden Phasen der Begriffsbildung auszuschalten und sie dann wieder zuzulassen. Dies ist m.E. die Hauptaufgabe des AnalysisUnterrichts,... “'129

Hier kann man nun einen Unterschied zwischen Benders und dem hier gezeigten Ansatz sehen: Geht es um das Verständnis des Grenzwertbegriffs bei Folgen (nicht um eine Vermutung, was Grenzwert sein könnte), lehnt Bender jede dynamische Vorstellung ab. Zur intuitiven Erfassung der Bedeutung des Lückenwerts gehört hier die Betrachtung der Differenzenquotientenfunktion einer Funktion $f$ an einer Stelle $x_{0}$, wobei man mit x (dynamisch!) gedanklich mehrmals über die Definitionslücke $x_{0}$ hinund herwandern sollte, um anhand von Überlegungen „Wie genau sind die Werte der einzelnen Durchschnittsgeschwindigkeiten bzgl. der Momentangeschwindigkeit?" zu schließen, dass der Lückenwert den Wert der Momentangeschwindigkeit angibt. Dazu muss hier sowohl eine Umgebung in der Definitionsmenge als Ganzes ins Auge genommen werden, als auch eine Umgebung der Wertemenge der Funktion $f^{\prime}$ an besagter Stelle (die Geschwindigkeit-Zeit-Funktion f' existiert intuitiv und liefert die Referenzmenge, in die der Lückenwert eingebettet wird). Das Zusammenspiel der Vorstellung von den Umgebungen als Ganzes mit der dynamischen Vorstellung des Hin- und Herwanderns soll bei diesem Ansatz Vorteile beim Entwickeln des Grenzwertbegriffs am Diffe renzenquotienten verschaffen.

Die Möglichkeit des Wanderns in der Definitionsmenge über die „kritische Stelle“ hinaus ist zentral bei dieser Überlegung. Diese Art von Wanderung ist bei Folgen nicht möglich! Wie sollte man zuerst bis zu einer Zahl $\infty$ gelangen und, wenn das ginge, über diese hinaus und wieder zurück? Damit steht der unüberwindliche Unterschied zwischen Benders und dem hier gezeigten Ansatz fest: Der Unterschied liegt in der Beschaffenheit der beteiligten mathematischen Objekte, insbesondere der Definitionsmengen begründet. Nur wenn endliche Intervalle betrachtet werden, wie bei dem Bewegungsproblem, kann man hin- und herwandern. Das gilt auch für den Fall des einseitigen Grenzwerts, bei dem man sich immerhin gedanklich der zu untersuchenden Stelle $x_{0}$ beliebig nähern kann. Sobald man aber unendliche Intervalle betrachten muss ( $\lim _{x \rightarrow \infty}$ bei Funktionen bzw. $\lim _{n \rightarrow \infty}$ bei Folgen), ist dieses Nähern in der Definitions menge nicht mehr möglich. Da die für Folgen typische Problematik mit den

${ }^{129}$ [Bender 1991b], S. 239, Hervorhebungen wie im Original 
natürlichen Zahlen (die sich nicht in einem endlichen Intervall einschließen lassen) hier nicht auftritt, kann man gegen die dynamische Vorstellung des Hin- und Herwanderns nichts einwenden.

1991 beginnt Bender seinen Artikel mit den Worten „Auch wenn Folgen und Grenzwerte in den Analysis-Kursen heutzutage knapper behandelt werden, spielt die Begrifflichkeit nach wie vor eine zentrale Rolle im Zusammenhang mit infinitesimalem Denken. “ ${ }^{130}$ Später fährt er fort:

\begin{abstract}
„Allerdings stellt der Abbau der ersten beiden Gebiete [Folgen / Grenzwerte und Stetigkeit, d. A.] eine didaktische Milchmädchenrechnung dar, jedenfalls wenn man meint, daß sich ohne einen eigens und ordentlich behandelten Grenzwertbegriff (in irgendeiner Form) als Fundament später mehr als nur vage und letztlich falsche und ungeeignete Vorstellungen und Verständnisse von zentralen Fragen, Methoden und Begriffen der Analysis wie lokale Änderungsrate u. v. a. m. ausbilden.“131
\end{abstract}

Interessant wären Untersuchungen und Diskussionen, ob der hier angeführte Zugang zum Grenzwertbegriff am Differenzenquotienten ebenfalls zu brauchbaren Vorstellungen und Verständnissen desselben führen könnte, die dann anders herum die Begriffsbildung des Grenzwerts von Folgen unterstützen können, die im Curriculum ${ }^{132}$ nur noch eine Ergänzung darstellen und z.B. nach den Ableitungsregeln oder nach der Integralrechnung behandelt werden können. Es ist denkbar, dass Schülerinnen und Schülern die Sichtweise „als Ganzes“, angewendet auf endliche Intervalle, leichter fällt, als wenn sie bzgl. der natürlichen Zahlen, die sich nicht in ein endliches Intervall einschließen lassen, benutzt werden soll.

\footnotetext{
${ }_{130}^{130}$ [Bender 1991b], S. 238

131 [Bender 1991b], S. 239

132 vgl. [NRW 1999]
} 


\section{Transkriptanalysen}

\subsection{Transkript 06}

Datum

Ort

Teilnehmerinnen
: 30.5.1999, 16:15-17:12

: Büro D2.326 in der Uni Paderborn

Aus dem Transkript 06 soll die Partnerarbeitsphase zu Aufgabenteil c) analysiert werden. Dazu wurde diese Arbeitsphase in fünf Szenen unterteilt. In der ersten Szene klären $\mathrm{K}$ und $\mathrm{C}$ für sich die Begriffe Durchschnittsgeschwindigkeit und Momentangeschwindigkeit. In der zweiten Szene zeigen sie, vor welchem Erfahrungshintergrund sie die Aufgabenstellung interpretieren, was ihre wichtigsten Argumentationsmuster für und wider die Existenz der Momentangeschwindigkeit sind und was für sie diese Existenz überhaupt heißt. Dabei gerät $C$ in einen Konflikt widersprüchlicher Argumentationen (Grundparadoxon). Szene 3 wird u.a. zeigen, wie auch $\mathrm{K}$ in diesen Konflikt kommt. In der vierten Szene beschäftigen die Mädchen sich u.a. mit der Frage, welche der beiden Geschwindigkeiten die grundlegendere ist. Die Analyse der letzten Szene zeigt, wie die Argumente präzisiert werden und was K unter „unendlich kleinen Einheiten“ versteht.

Über die gesamte Arbeitsphase ändern die Mädchen ihre Meinungen, ob es Momentangeschwindigkeit gibt, oder nicht. Es wird versucht, diese Veränderung nachzuzeichnen und nachzuvollziehen.

Zunächst wird kurz zusammengefasst, wie C und K die Aufgabenteile a) und b) bearbeiteten.

\section{Aufgabenteile a) und b)}

Die Bearbeitung des Aufgabenteils a) bereitet $\mathrm{C}$ und $\mathrm{K}$ einige Probleme. Zuerst versuchen sie den Funktionsterm zu interpretieren. Es kommt ihnen komisch vor, dass 
dieser eine 5 im Nenner enthält, sie aber eine Entfernung für 3 Stunden berechnen sollen. Dann setzen sie den Term mit Null gleich und wollen inn nach $t$ auflösen. Schließlich setzen sie für $t$ den Wert 3 ein.

Der Aufgabenteil b) bereitet innen keine Schwierigkeiten. Sofort erkennen sie, dass die Formulierung "Durchschnittsgeschwindigkeit der letzten $x$ Stunden" die Berechnung einer Wegdifferenz erfordert.

Nach insgesamt ca. 17 Minuten Arbeitszeit decken sie den Aufgabenteil c) auf:

\section{Szene 1; Zeilen 160 - 175}

160 Beide lesen die Aufgabe, holen Blatt b) wieder hervor.

161 C: 16 Uhr. Das war die letzte Stunde, ne? ... Ja doch, ja aber

162 K: Ehm, aber um 16 Uhr, genau um 16 Uhr...

163 C: Ja, haben wir jetzt hier eigentlich eben auch die Durchschnittsgeschwindigkeit 164 berechnet oder gehabt? (meint b))

165 K: Ja, also hier ja, also Durchschnittsgeschwindigkeit (C: Ja.) ... ja ... (nuschelt)

166 C: Ja, dann haben wir doch, das ist doch dann, ... wenn wir das genauso aus gerech167 net haben, dann muss das doch was anderes sein, als das, weil wir den Durch168 schnitt-Momentangeschwindigkeit gesprochen.

169 K: Ja.

170 C: Das kann ja nicht, das ist doch dann die Momentangeschwindigkeit. (zeigt auf b))

171 K: Nein, das ist die Durchschnittsgeschwindigkeit.

172

173 (17:45) K erklärt, was sie unter dem Begriff Durchschnittsgeschwindigkeit versteht. C 174 stimmt ihr zu. (18:10)

C setzt Momentangeschwindigkeit mit Durchschnittsgeschwindigkeit gleich
Nachdem $\mathrm{C}$ und $\mathrm{K}$ die Aufgabe gelesen haben, vergleichen sie diese mit dem Aufgabenteil b). C bezieht die Zeitangabe 16 Uhr auf Aufgabe b) (Zl. 161): "16 Uhr. Das war die letzte Stunde, ne?" und versucht auch den Begriff "Durchschnittsgeschwindigkeit" dem Aufgabenteil b) zuzuordnen (ZI. 163 - 164). Sie erkennt einerseits, dass es einen Unterschied zwischen 
den Aufgabenteilen b) und c) geben muss, andererseits ist inr der Unterschied zwischen Durchschnittsgeschwindigkeit und Momentangeschwindigkeit nicht klar (Zl. 166 - 168, 170). K scheint zumindest der Durchschnittsgeschwindigkeitsbegriff klar zu sein. Sie erklärt $C$, was sie unter Durchschnittsgeschwindigkeit versteht. Weiterhin denkt sie gleich von Beginn an über die Bedeutung des Zeitpunkts (für die Momentangeschwindigkeit?) nach, vgl. Zl. 162.

\section{Szene 2; Zeilen 176 - 199}

176

C: (K murmelt, C zeigt auf Funktionsgleichung) Ja dann müssen wir das einfach durch irgendwas rechnen, weil ... (Pause 7s) ...

K: (nuschelt, dann) Momentangeschwindigkeit heißt ja, dass er in dem Moment so viel fährt. ... Also müsste es so was geben. Ob man, wie man so etwas aus rechnet..? (nuschelt, C: Ja.) ... Hier steht ja eigentlich auch gar kein nicht, keine Rechenaufgabe. Hier steht nur, gibt es auch so etwas wie eine Momentangeschwindigkeit um 16 Uhr? ... Und wenn ja, begründet. Ja, er muss ja ne,... ne bestimmte Geschwindigkeit draufgehabt haben.

C: Ja, das ist klar. Also, er muss eine gehabt haben. Ich meine, durchschnittlich könn wir das ausrechnen, würd ich das auch sagen (unverständlich)

$\mathrm{K}$ : Wie viel er genau gefahren ist, willst du schreiben?

C: Die Momentangeschwindigkeit ...

K: Wenn das..

C: Ja genau um 16 Uhr kann man das ja eigentlich nicht rechnen, weil er genau um 16 Uhr (macht eine Armbewegung, die wohl eine Bewegung andeuten soll), $16 \mathrm{Uhr}$ ist ja nicht lange, ne? Ist ja nur ne Sekunde...

$\mathrm{K}$ : (unterbrechen sich gegenseitig) Ja, wenn das jetzt...

C: Ja das ist..

K: Er fährt ja durch. Er unterbricht (???) ja nicht die Fahrt. Ja ehm, das sind, in jeder Sekunde muss er ja ne bestimmte Geschwindigkeit draufhaben. ... Kann man ja bis in die Tausendstel (unverständlich)

C: Also in sofern kann's das ja dann nicht geben, also,... kannst aber nicht ... anhalten ... Oder du musst durch irgendwas rechnen, dann geht's ja. ... Aber du kannst ja nicht genau ausrechnen, wie viel er genau in dieser Sekunde fährt. ... 
C's und K's Vorerfahrungen mit den Geschwindigkeitsbegriffen

\section{Erfahrungshintergründe}

In diesem Abschnitt soll analysiert werden, in welchen Zusammenhängen $\mathrm{K}$ und $\mathrm{C}$ den Begriff Momentangeschwindigkeit kennen, welchem Kontext sie inn zuschreiben. Beiden scheint das Wort Momentangeschwindigkeit bekannt zu sein. Sie verwundern sich nicht über dieses Wort. C möchte sofort den Wert der Momentangeschwindigkeit berechnen, wie man ihren ersten beiden Redebeiträgen (ZI. 176 - 177; 184 - 185) entnehmen kann. Vielleicht kennt sie dieses Wort aus einem mathematisch-physikalischen Zusammenhang. Vielleicht zeigt sich hier auch nur, dass sie versucht, der Erwartung, die sie dem Versuchsleiter ihr gegenüber zuschreibt, gerecht zu werden. C weiß, dass der Versuchsleiter „,von der Mathematik an der Universität" kommt, und dass sie an einer mathematischen Untersuchung teilnimmt. Zudem wurde sie in einer Mathe matikstunde in der Schule durch den Versuchsleiter rekrutiert. C's Sicht: Es geht hier um Mathematik, also muss gerechnet werden. Ihre Formulierung „Ja dann müssen wir das einfach durch irgendwas rechnen,..." (ZI. 176 - 177) zeigt an, dass sie die in Aufgabenteil b) erfolgreich genutzte Strategie "Weglänge / Zeitdauer“ weiter nutzen will.

Während $C$ den Begriff der Momentangeschwindigkeit eher einem physikalisch-mathe matischen Kontext ( $m$-Welt) zuschreibt, deren Wert zu berechnen ist, erkennt $\mathrm{K}$, dass die Aufgabenstellung eine Berechnung der Momentangeschwindigkeit gar nicht erfordert (ZI. 180 - 182). Sie scheint den Begriff zunächst aus dem Wort selber heraus zu interpretieren (Zl. 178 179): „Momentangeschwindigkeit heißt ja, dass er in dem Moment so viel fährt." Die Formulierung "dass er so viel fährt" zeigt an, dass sie sich den Radfahrer vorstellt. Sie interpretiert den Begriff Momentangeschwindigkeit in der Erfahrungswelt, in der r-Welt.

\section{Argumentationsmuster}

Hier sollen die beiden Argumentationsmuster von $\mathrm{C}$ und $\mathrm{K}$ aufgezeigt werden, mit denen sie begründen, warum es die Momentangeschwindigkeit gibt, bzw. nicht gibt. 
Zunächst zu C:

Durchschnittsge- In den Zeilen 184 und 185 antwortet $C$ auf ein Argument für die Existenz schwindigkeit in $r$ - und $m$-Welt der Momentangeschwindigkeit von $\mathrm{K}$ : „Ja, das ist klar. Also, er muss eine gehabt haben. Ich meine, durchschnittlich könn wir das ausrechnen, würd ich das auch sagen." Auf den ersten Blick sieht es so aus, als ob $\mathrm{C}$ die Existenz der Momentangeschwindigkeit in der r-Welt befürwortet („er muss eine gehabt haben"), sie aber in der m-Welt nicht berechnen kann (,durchschnittlich könn wir das ausrechnen“). Aber in der Analyse von Szene 1 wurde gezeigt, dass $C$ die Bedeutung der Begriffe "Durchschnittsgeschwindigkeit" und "Momentangeschwindigkeit" noch vermischt. Nach wie vor könnte sie hier unter beiden Begriffen die Durchschnittsgeschwindigkeit verstehen. Unter dieser Voraussetzung existiert für sie die Durchschnittsgeschwindigkeit in beiden Welten: „er muss eine gehabt haben“, „durchschnittlich könn wir das ausrechnen“, nämlich wie in Aufgabenteil b). K's Argumente für die Existenz der Momentangeschwindigkeit, denen sie zustimmt, sind eher der r-Welt zuzuordnen (s.u.). C's Schlussfolgerungen passen in der $\mathrm{F}$ und $\mathrm{m}$-Welt zusammen: Die Momentangeschwindigkeit (was auch immer sie darunter versteht) gibt es, und man kann ihren Wert berechnen: Also „würd ich das auch sagen.“ (ZI. 185) Das „auch“ bezöge $C$ auf sich selber: Auch ich bin der Meinung. Das „auch“ kann sich aber ebenso auf "durchschnittlich“ (ZI. 185) in folgendem Sinn beziehen: Momentangeschwindigkeit gibt es ja, aber auch Durchschnittsgeschwindigkeit, die kann man nämlich berechnen. In diesem Fall würde $C$ zwischen Durchschnittsgeschwindigkeit und Momentangeschwindigkeit unterscheiden.

Andererseits könnte C's Antwort auch so gemeint sein: Ja, die Momentangeschwindigkeit gibt es auch, aber ausrechnen können wir nur die Durchschnittsgeschwindigkeit. Wahrscheinlich ist ihr schon klar, dass es einen Unterschied gibt, auch wenn sie den Begriff der Momentangeschwindigkeit noch nicht richtig fassen kann. Wichtig für die Frage nach der Existenz der Momentangeschwindigkeit scheint ihr die Berechenbarkeit zu sein. Durchschnittsgeschwindigkeit gibt es, da man sie berechnen kann (ZI. 184 185). Die Momentangeschwindigkeit möchte sie auch berechnen (ZI. 176 - 
177). Das wird auch an ihrem folgenden Redebeitrag deutlich (ZI. 189 191):

C will die Momentangeschwindigkeit berechnen

C's Existenzbegriff

Der Zeitpunkt ist Ursache von C's Berechnungsproblem in der m-Welt
"Ja genau um 16 Uhr kann man das ja eigentlich nicht rechnen, weil er genau um $16 \mathrm{Uhr}$, [...] $16 \mathrm{Uhr}$ ist ja nicht lange...", und (ZI. 197): "Also in sofern kann's das ja dann nicht geben,..." Dass sich $C$ hier auf die Momentangeschwindigkeit bezieht und nicht auf die Durchschnittsgeschwindigkeit, kann man so begründen: In Aufgabenteil b) hat sie gezeigt, dass sie die Durchschnittsgeschwindigkeit berechnen kann. Wenn also eine Geschwindigkeit nicht berechenbar ist, dann die Momentangeschwindigkeit.

Unterstellt man C die Alltagsvorstellung "Genau wenn sich ein Körper bewegt, hat er auch eine (positive) Momentangeschwindigkeit." und fügt hinzu "Genau wenn ein Körper eine (positive) Momentangeschwindigkeit hat, ist diese berechenbar.", so verwendet sie hier die Argumentations richtung: "Weil die Momentangeschwindigkeit nicht berechenbar ist, gibt es sie nicht." In dieser Argumentation vermischen sich Argumente aus der $\mathrm{r}$ - und $\mathrm{m}$-Welt, da man einerseits die Vorstellung des sich bewegenden Körpers bemüht und andererseits die Frage nach der Existenz aufgrund der Berechenbarkeit entschieden wird. Offen bleibt bis hier, warum $\mathrm{C}$ die Momentangeschwindigkeit für nicht berechenbar hält. Man kann aber erkennen, dass das mit dem Zeitpunkt 16 Uhr zusammenhängen muss.

C behauptet, dass man die Momentangeschwindigkeit um genau $16 \mathrm{Uhr}$ nicht berechnen kann (ZI. 189). Dabei formuliert sie "Man kann nicht" und nicht "Ich kann nicht". Das deutet darauf hin, dass die Nicht-Berechenbarkeit für sie ein prinzipielles und kein persönliches Problem zu sein scheint. Das ist nicht uninteressant, wenn man die Unsicherheiten bei der Bearbeitung der Teilaufgabe a) berücksichtigt. Dann versucht sie zu erklären, warum die Berechnung prinzipiell nicht möglich ist. Dazu macht sie eine Armbewegung, um eine Bewegung um 16 Uhr zu verdeutlichen (Zl. $190-$ 191): "16 Uhr ist ja nicht lange, ne? Ist ja nur eine Sekunde..." Die Armbewegung soll vielleicht die Vorstellung von einer Bewegung unterstützen. C müsste aus Aufgabenteil b) klar sein, dass sie die Durchschnittsge- 
Die Identifikation von „Zeitpunkt“ und "Sekunde“

Die Armbewegung klärt nicht auf

schwindigkeit für den Zeitraum einer Sekunde berechnen kann. Wahrscheinlich verwendet sie hier "Sekunde" und "Zeitpunkt" synonym, da ihr Kommentar ja ein Argument gegen die Berechenbarkeit der Momentangeschwindigkeit sein soll. - Die Identifikation von Zeitpunkt und Sekunde kann verschiedene Gründe haben. Zum einen ist die Maßeinheit Sekunde die kleinste Einheit für Zeit im Alltagsgebrauch (,pünktlich auf die Sekunde") und wird daher mit dem Zeitpunkt identifiziert. Hinzu kommt die Art und Weise, wie man Zeitpunkte im Alltag aufschreibt, z.B. 16:00:00. Erst für den Zeitpunkt 16:00:01 hat man eine neue Schreibform. Daran könnte es liegen, dass der Zeitpunkt 16:00:00 mit dem Zeitraum 16:00:00 16:00:01 identifiziert wird. Interessant für die Mathematik- und Physikdidaktik wäre die verallgemeinerte Frage, ob sich Jugendliche des „Digitalzeitalters" verschiedene kontinuierliche Größen zunehmend digitalisiert oder diskretisiert vorstellen. In diesem Zusammenhang könnte man die Frage untersuchen, ob Jugendliche Größen, die in der jeweils kleinsten (alltagsgebräuchlichen) Einheit gegeben sind, mit „unendlich klein“, identifizieren.

Die Armbewegung kann zur Klärung der Frage, ob Sekunde und Zeitpunkt identifiziert werden, nicht beitragen. $C$ könnte die Armbewegung wie folgt verstehen: Man kann hier nur die Durchschnittsgeschwindigkeit eines kurzen Zeitraums (Sekunde) berechnen (ganze Armbewegung), oder: Die Hand befindet sich zu jedem Zeitpunkt der Armbewegung an genau einem Ort.

Das 0/0-Problem; Hinweis auf die isolierende Sichtweise
C's Behauptung, dass man die Momentangeschwindigkeit nicht berechnen kann, kann als Indiz für die Identifikation von Sekunde und Zeitpunkt gesehen werden. Wie oben gezeigt, will sie die erfolgreiche Strategie "Weglänge / Zeitdauer" fortsetzen. Für Durchschnittsgeschwindigkeiten mit Weglängen größer Null in einem Zeitraum bereitet das kein Problem. Da es aber für Zeitpunkte keine lokale Änderung des Orts und der Zeit gibt, könnte sie auf das Problem gestoßen sein, dass sowohl Zähler als auch Nenner ihres Quotienten Null sind, und daher eine Berechnung nicht möglich ist. Das 0/0-Problem, das sie hier noch nicht explizit formuliert, kann aber als Indiz für die isolierende Sichtweise in der $\mathrm{F}$ und in der $\mathrm{m}$ - 
Welt angesehen werden (vgl. 3.2 „Momentangeschwindigkeit“ und 4.3): In der r-Welt wird die momentane Weg- und Zeitänderung in den Vordergrund gestellt, der Rest der Bewe gung (die Bewegung als Ganzes) ausgeblendet. Das hat direkte Auswirkungen auf die m-Welt, wenn die Grenzwerte für Zähler und Nenner des Differenzialquotienten gesucht werden, wegen der isolierenden Sicht nicht gefunden werden können und daher gleich Null gesetzt werden. Einen Grenzprozess in der m-Welt als Ganzes (einbettend) zu betrachten bedeutet zudem, den Differenzen- und den Diffe renzialquotienten als Ganzes zu sehen und nicht Zähler und Nenner getrennt. Denn dann kann man erkennen, dass der Grenzwert der Differenzenquotientenfolge einen Wert annimmt, der auch ungleich Null sein kann. Für $C$ steht aber der Zeitpunkt 16 Uhr stark im Vordergrund, so stark, dass sie vielleicht den Zähler aus den Augen verliert, ganz bestimmt aber den Bewegungsvorgang als Ganzes. Die isolierende Sicht in der $\mathrm{FWelt}$ zeigt hier eine große Auswirkung auf die Vorstellungen in der $\mathrm{m}$-Welt. $\mathrm{C}$ stellt einen Zusammenhang zwischen den Welten her. - Nimmt man die isolierende Sichtweise ein, werden Zeitpunkt und Zeitraum voneinander isoliert gesehen. Bis hier wurde auf die Betonung des Zeitpunkts eingegangen. C's Formulierung (ZI. 191) „ist ja nur eine Sekunde“ könnte auf eine ZeitraumBetonung hinweisen. Jedoch aufgrund der Identifikation von Zeitpunkt und Sekunde erscheint dies als unwahrscheinlich.

C's r-Welt-Überlegungen; einbettende Sichtweise
Von $\mathrm{K}$ angeregt, kann $\mathrm{C}$ aber auch die einbettende Sichtweise einnehmen. $\mathrm{K}$ bietet ihr eine r-Welt-Vorstellung in einbettender Sichtweise an (ZI. 194 196): "Er fährt ja durch. Er unterbricht (???) ja nicht die Fahrt. Ja ehm, das sind, in jeder Sekunde muss er ja ne bestimmte Geschwindigkeit draufhaben. ... Kann man ja bis in die Tausendstel (unverständlich)". C antwortet (Zl. 197 - 198): "Also in sofern kann's das ja dann nicht geben, also, ... kannst aber nicht ... anhalten".

$\mathrm{K}$ benutzt die Einheit tausendstel Sekunde. Wahrscheinlich setzt $\mathrm{C}$ das wieder mit einem Zeitpunkt gleich, denn dann kann ihre Schlussfolgerung (ZI. 197) „Also in sofern kann's das ja dann nicht geben“ mit den oben angeführten Argumenten als für sie schlüssig angesehen werden. Dann 
C gerät in einen Widerspruch

greift C K's Überlegung in der einbettenden Sichtweise auf: Nicht-AnhaltenKönnen entspricht K's Durchfahren, was den Bewegungsvorgang über einen Zeitraum in den Blick rückt. C's Formulierung (Zl. 198) „... kannst aber nicht ... anhalten" zeigt, dass sie hier ein Argument hat, das gegen ihre zuvor geäußerte Meinung (die Momentangeschwindigkeit gibt es nicht) spricht. Dieses neue Argument lautet so: Wenn es die Momentangeschwindigkeit nicht gäbe, würde keine Bewegung stattfinden. Also müsste die Bewegung des Radfahrers für einen Zeitpunkt unterbrochen worden sein, er müsste angehalten haben. Da der Radfahrer sich aber insgesamt bewegt (er befindet sich auf seiner Radtour), kann er nicht angehalten haben.

Zenons PfeilParadoxon

$\mathrm{C}$ und $\mathrm{K}$ haben hier letztlich das Pfeil-Paradoxon von Zenon für sich formuliert. Es kann so gesehen werden, dass eine kontinuierliche Bewegung nicht aus Bewegungen zu Zeitpunkten zusammengesetzt werden kann. Sehr deutlich bringt $C$ das Grundparadoxon in der schriftlichen Bearbeitung zu Papier (vgl. Abbildung 9): "Nein, da man nur wenn man in Bewegung ist eine Geschwindigkeit erreichen kann. Wenn man aber in Bewegung ist, läuft dies in einem Zeitraum ab und man legt einen Weg zurück. Deswegen gibt es nur eine Durchschnittsgeschwindigkeit." Hier zeigt sich die Betonung des Zeitraums bei der isolierenden Sichtweise. Man kann vermuten, dass die Existenz der Momentangeschwindigkeit mit einem positiven Wert gleichgesetzt wird.

C's Rückkehr in die $\mathrm{m}$-Welt

Das Grundparadoxon in den Denkwelten

Dieser Widerspruch, der durch $\mathrm{F}$ Welt-Überlegungen offenbar wurde, veranlasst $\mathrm{C}$ vielleicht, wieder zur $\mathrm{m}$-Welt zurückzukehren, was ihr aber auch nicht weiterhilft (Zl. 198 - 199): ,... Oder du musst durch irgendwas rechnen, dann geht's ja. ... Aber du kannst ja nicht genau ausrechnen, wieviel er genau in dieser Sekunde fährt. ..." Das „durch etwas Rechnen“ bringt sie bzgl. der Momentangeschwindigkeit sofort wieder zum 0/0-Problem.

Es zeigt sich hier das Grundparadoxon, allerdings nicht innerhalb einer Denkwelt: In der m-Welt gibt es für $\mathrm{C}$ keine Momentangeschwindigkeit, da man sie nicht berechnen kann. In der r-Welt gibt es sie, da der Radfahrer nicht anhält. Die Folgerungen aus Vorstellungen der Denkwelten passen 
nicht zusammen. Das liegt daran, dass C zwei verschiedene von den Denkwelten abhängige Existenzbegriffe hat. Zum einen entscheidet sie aufgrund der Berechenbarkeit „Was man nicht berechnen kann, gibt es nicht..", zum anderen aufgrund der Alltagserfahrung „Nur was sich bewegt, hat Momentangeschwindigkeit, also gibt es die."

c) Bisher war immer von Durchschnittsgeschwindigkeiten die Rede. Gibt es auch so etwas wie eine Momentangeschwindigkeit um 16:00 Uhr? Begründe!

Ja, abes mur weun mare den Punkt hat anden das $\operatorname{lad}$ un 16:00 uhr derergefahrer ist mad auf wich kluinste sinheit unredinet.

Nein, da man nor wenn man in Dewegung ist aine Gesdwindigkeit erreichen kann. Wenn man in Bewegung ist, läuft dies in cinem Zeitraum ab und man lest einen los wrück. Deswegen sibt es nor line Durchroluittgestowindiskeit.

Abbildung 9: Zwei alternative Antworten zu Aufgabenteil c

C's Wechsel und Zusammenhänge der Denkwelten, Überblick
Gedanklich startet C in der m-Welt (ZI. 176 - 177): Sie will die Momentangeschwindigkeit berechnen. Es sieht so aus, als könne die Entstehung und Festigung der isolierenden Sichtweise der m-Welt zugeschrieben werden, da $C$ in beiden Welten argumentiert, sich die einbettende aber nur in der $\mathrm{r}-$ Welt nachweisen lässt. In Zl. 184 bestätigt sie K's r-Welt-Argument (einbettende Sichtweise), fragt aber sofort nach einer Berechnungsmöglichkeit in der m-Welt. Genauso bringt sie in ihren beiden folgenden Redebeiträgen die Welten in einen Zusammenhang: ZI. 189 - 191: Armbewegung als 
Unterstützung einer r-Welt Vorstellung mit der Feststellung, dass „Ja genau um 16 Uhr kann man das ja eigentlich nicht rechnen" (isolierende Sichtweise). Ebenso in den Zeilen 197 - 199: „nicht anhalten können“ (einbettende Sichtweise) in der r-Welt wird dem "nicht rechnen können in genau dieser Sekunde" (isolierende Sichtweise) in der m-Welt gegenüber gestellt.

Nun zu K:

K's Argumente in Nachdem K in Szene 1 den Unterschied zwischen den Teilaufgaben a) und der r-Welt; einbettende Sichtweise b) analysiert hat, überlegt sie nun, was sie unter dem Wort Momentangeschwindigkeit verstehen will und ob es diese gibt (ZI. 178 - 183): "Momentangeschwindigkeit heißt ja, dass er in dem Moment so viel fährt.... Also müsste es so was geben. Ob man, wie man so etwas aus rechnet..? [...] ... Hier steht ja eigentlich auch gar kein nicht, keine Rechenaufgabe. Hier steht nur, gibt es auch so etwas wie eine Momentangeschwindigkeit um 16 Uhr? ... Und wenn ja, begründet. Ja, er muss ja ne,... ne bestimmte Geschwindigkeit draufgehabt haben."

Für sich beantwortet sie die Frage nach der Existenz der Momentangeschwindigkeit sofort positiv: „Also müsste es so was geben.“ Die Formulierung "...dass er in dem Moment so viel fährt." lässt darauf schließen, dass sie sich den Radfahrer vorstellt, gedanklich wahrscheinlich in der $r$-Welt ist. Dann will sie vielleicht C's Frage nach der Berechenbarkeit aufgreifen (Zl. 179): „Ob man, wie man so etwas aus rechnet..?“, blockt die Frage aber ab. Ihre Analyse der Aufgabe führt nämlich zu dem Ergebnis, dass die Berechnung der Momentangeschwindigkeit nicht gefordert wird. Sie bekräftigt noch einmal, dass es die Momentangeschwindigkeit gibt, wobei sie sich wieder auf den Radfahrer bezieht. $C$ setzt ihre Überlegungen bezüglich der

$\mathrm{K}$ will nicht in die m-Welt

einbettende Sichtweise Berechenbarkeit fort und lenkt K's Aufmerksamkeit auf kleine Zeiträume oder Zeitpunkte (ZI. 189 - 191). K will oder kann sich zunächst aufgrund einer deutlich ausgeprägten r-Welt-Sicht auf C's Problem nicht einlassen. Ihre dort einbettende Sichtweise wird an der Zeile 194 deutlich, die zudem als Begründung für K's Meinung gesehen werden kann, dass es eine Momentangeschwindigkeit gibt: "Er fährt ja durch. Er unterbricht (???) ja nicht die Fahrt." Unabhängig von der Radfahrsituation lautet ihre Argumentation 
so: Ein Körper, der sich in einem gewissen Zeitraum bewegt, hat zu jedem Zeitpunkt des Zeitraum eine positive Momentangeschwindigkeit.

An dieser Stelle ist $\mathrm{K}$ für sich zu einer Antwort mit einer für sie logischen Begründung gekommen. Vielleicht trägt dieser gedankliche Abschluss dazu bei, dass sie sich nun C's Problem der Berechnung zuwenden kann (ZI. 194): "Ja ehm, das sind, [...]". Eine konkrete Antwort kann sie jedoch nicht liefern. Vielleicht erkennt sie schon ansatzweise C's inneren Konflikt und flüchtet auf sicheren Boden zurück, zu ihrer logischen Argumentation (ZI. 195): "in jeder Sekunde muss er ja ne bestimmte Geschwindigkeit draufhaben....".

K sieht „Zeitpunkt" und "Se kunde" differenzierter
Man kann hier sehen, dass sie aber auf C's Wortwahl "Sekunde“ eingeht: Vielleicht versteht auch $\mathrm{K}$ in diesem Sprechabschnitt unter einer Sekunde einen Zeitpunkt. Kurz darauf zeigt sich jedoch, dass für $\mathrm{K}$ auch kürzere Zeiteinheiten eine Rolle spielen (ZI. 195 - 196): "... Kann man ja bis in die Tausendstel". Daher klappt die oben angeführte Argumentation „Sekunde = Zeitpunkt, da Sekunde = kleinste Zeiteinheit des Alltags" nicht. Diese gedankliche Entwicklung von Sekunden zu tausendstel Sekunden kann man vielleicht als Beginn eines Grenzprozesses sehen. In dem Fall will wohl auch K die erfolgreiche „Weglänge / Zeitdauer"-Strategie aus Aufgabenteil b) übernehmen und überlegt, wie genau sich die Momentangeschwindigkeit berechnen lässt.

\section{Entscheidungsgrundlagen}

Wie schon angedeutet, treten in dieser Szene zwei Entscheidungsgrundlagen zu Tage, die die Basis der Entscheidung der Frage nach der Existenz der Momentangeschwindigkeit bilden. In der r-Welt ist die Basis die Alltagsvorstellung: Bewegte Körper haben eine positive Momentangeschwindigkeit. In der m-Welt stellt die Berechenbarkeit die Basis: Nur wenn man die Momentangeschwindigkeit berechnen kann, gibt es sie auch. C, die Überlegungen in beiden Welten anstellt, kommt bezüglich der verschiedenen Entscheidungsgrundlagen zu verschiedenen Antworten und gerät in einen inneren Konflikt. 


\section{Szene 3; Zeilen 200 - 217}

200 K: Hmm... Ja, ob man's ausrechnen kann, weiß ich nicht. ... Nur, ich mein ja, hier 201 steht ja nicht, wir müssen ja hier nichts rechnen. Es ist, es gibt, es ist ja hier nur 202 die Frage, ob es so was gibt, oder ob's so was nicht gibt. ... Ich weiß es nicht. Ich 203 würd sagen, es gibt so was, nur ... Aber obwohl, meistens so nen Tachometer und 204 so rechnen auch immer mit Durchschnittsgeschwindigkeiten. ... (murmeln, Pause 205 19s) ... Moment, Tausendstel ... kleinen Moment ...

206 C: (flüstert) Weitermachen (meint nächsten Zettel)

207 K: Ja? Wart erst mal. Ist ja eigentlich ... Ist logisch, wenn'st Auto fährst..

208 C: Wenn'st Auto fährst..

209 K: (lacht) Ja, ok, wenn du Auto fährst

210 C: Kommst aus Bayern?

$211 \mathrm{~K}: \mathrm{Ne}$, ich komm nicht aus Bayern. Aber wenn du Auto fährst, dann fährst du 50,...

212 fährst du 60 (lacht), ich versuche mir das irgendwie vorzustellen, weil ... wie der

213 Tachometer da reagiert. Er misst ja, sagen wir mal in $100 \mathrm{~m}$, ich weiß nicht ob 214 (nuschelt) in 100, in Kilometern, ... wie viel man, ... wie schnell man ist und ... 215 muss ja auch irgendeine Geschwindigkeit, beziehungsweise und rechnet das dann 216 um. ... Aber er gibt die auch nicht ... ehm so ganz genau die Geschwindigkeit, ne 217 keine Ahnung.

\section{$\underline{\text { K's Weg in den inneren Konflikt }}$}

In C's vier längeren Redeabschnitten der vorherigen Szene wirft diese immer wieder die Frage nach der Berechenbarkeit auf: ZI. 177, 185, 189, 198, 199. Bei der ersten Stelle reagiert K so (ZI. 179 - 181): „Ob man, wie man so etwas aus rechnet..? (nuschelt, C: Ja.) ... Hier steht ja eigentlich auch gar kein nicht, keine Rechenaufgabe." Einerseits weiß auch K nicht, ob und wie man die Momentangeschwindigkeit berechnen könnte, andererseits interessiert es sie auch nicht so sehr, weil die Aufgabenstellung eine Berechnung nicht verlangt. C's zweiten und dritten Versuch, auf das Berechnungsproblem aufmerksam zu machen, ignoriert sie mehr oder weniger. 
Der Tachometer als Messinstrument der Momentangeschwindigkeit
K's Vorstellungen, wie ein Tacho funk tioniert
Diese Szene beginnt damit, dass $\mathrm{K}$ auf den vierten Versuch wie beim ersten reagiert (ZI. 200 - 202): „Ja, ob man's ausrechnen kann, weiß ich nicht. ... Nur, ich mein ja, hier steht ja nicht, wir müssen ja hier nichts rechnen. Es ist, es gibt, es ist ja hier nur die Frage, ob es so was gibt, oder ob's so was nicht gibt.“ Wahrscheinlich hat sie schon gemerkt, dass $\mathrm{C}$ hier ein echtes Problem, das Grundparadoxon, aufwirft. Aus ihrer Sicht hat K aber eine logische Begründung dafür, dass es Momentangeschwindigkeit gibt: Der Radfahrer ist in Bewegung. Diesen sicheren Grund will sie vielleicht nicht verlassen. Daher versucht sie, ihre Position zu stärken, indem sie ein Alltagsinstrument zur Messung von Momentangeschwindigkeit heranzieht, einen Tacho (ZI. 203 - 204): „Aber obwohl, meistens so nen Tachometer und so rechnen auch immer mit Durchschnittsgeschwindigkeiten."

Ihre Äußerung, dass ein Tacho die Durchschnittsgeschwindigkeit berechnet, erstaunt ein wenig. Im anschließenden Gespräch, auf das in dieser Analyse nicht weiter eingegangen werden soll, fragte der Versuchsleiter nach und erfuhr, dass sich $\mathrm{K}$ zunächst einen digitalen Fahrradtacho vorstellte, wahrscheinlich auf Grund der in der Aufgabe angebotenen Radfahrsituation. In einer längeren Pause scheint $\mathrm{K}$ weiter über die Genauigkeit der Berechnungen nachzudenken, wobei kurze Zeiträume eine besondere Rolle spielen (Zl. 205). Digitale Fahrradtachos berechnen in der Regel die Durchschnittsgeschwindigkeiten für feste Zeitintervalle, üblicherweise der jeweils vergangenen Sekunde. Vielleicht zeigen diese Zeitintervalle $\mathrm{K}$ auf, dass diese Art von Tacho die Momentangeschwindigkeit nicht anzeigen kann. Daher wechselt sie gedanklich zu einem anderen Tachotyp, dem Zeigertacho im Auto, bei dem die Anzeige nicht so umspringt, wie beim digitalen Tacho. Offenbar ist sie der Überzeugung, dass auch dieser Tachotyp die Geschwindigkeit berechnet, da sie sich überlegt, nach wie viel zurückgelegten Metern eine neue Berechnung angestellt werden könnte (ZI. 213 - 214). Hier nimmt K Weglängen in den Blick (ZI. 213 - 215): „Er misst ja, sagen wir mal in $100 \mathrm{~m},[\ldots]$... wie schnell man ist und ... [...] und rechnet das um." Sie kommt zu dem Ergebnis, dass ein Tacho, der nach ihren Vorstellungen funktioniert, die Momentangeschwindigkeit nicht 
Die aus den Überlegungen zur Funktions weise des Tachos resultierende Sichtweise

anzeigen kann (Zl. 216 - 217): „Aber er gibt auch nicht ... ehm so ganz genau die Geschwindigkeit, ne keine Ahnung." Auch hier kann man das 0/0-Problem unterstellen. Die Fahrradtachos scheiden aufgrund einer Diskretisierung der Zeit, die Autotachos aufgrund einer Diskretisierung der Wegstrecke aus.

$\mathrm{K}$ stellt sich die Frage nach der $\mathrm{m}$-Welt-Methode, nach der ein Tacho die Momentangeschwindigkeit berechnet. Das lässt auch bei ihr die isolierende Sichtweise dominieren, obwohl sie in der r-Welt schon eingehend die einbettende Sichtweise verwendet hat. Da aufgrund ihrer Vorstellung der Funktionsweise eines Tachos dieses Instrument die Existenz der Momentangeschwindigkeit nicht, wie vielleicht erwartet, unterstützen kann, wird sie wohl verunsichert sein.

Bei C war es nicht klar, ob sie "Tausendstel" und "Zeitpunkt" synonym verwendet. In den Zeilen 196 und 205 denkt K über diese Begriffe nach (ZI. 205): "Moment, Tausendstel ... kleinen Moment". Ich interpretiere den Gedankengang so: "Sind ein Moment und eine Tausendstel dasselbe? - Nein, ein Tausendstel ist ein kleiner Moment, also ein Zeitraum." Das würde bedeuten, dass $\mathrm{K}$ sehr wohl einen Unterschied zwischen Zeitpunkt und Zeitraum sieht. Das wird in der folgenden Szene in den Zeilen 220 und 221 bestätigt.

Fazit: Auch K muss sich nun mit dem Grundparadoxon plagen. Es stehen sich zwei jeweils logisch erscheinende aber unvereinbare Argumentationen gegenüber.

a) Die Momentangeschwindigkeit gibt es, denn wenn sich eine Körper über einen Zeitraum bewegt, so hat er zu jedem Zeitpunkt des Zeitraums eine positive Momentangeschwindigkeit drauf. (vgl. Zl. 194 195; einbettende Sichtweise)

b) 0/0-Problem: Die Momentangeschwindigkeit gibt es nicht, denn zu einem Zeitpunkt bewegt sich ein Körper nicht, er legt keinen Weg zurück. Daher hat er auch keine Momentangeschwindigkeit drauf. Diese explizite Formulierung wurde bis hier von keinem der Mädchen verwendet, 
wird aber in der 5. Szene genannt und in der schriftlichen Antwort zu Aufgabenteil c) verwendet. (isolierende Sichtweise)

\section{C's erster Ausbruchsversuch}

Bis hier wurden bzgl. dieser Szene fast nur K's Gedanken analysiert. Das liegt daran, dass $C$ hier keinen inhaltlichen Beitrag zur Diskussion leistet. Es soll jetzt gezeigt werden, dass sie sogar versucht, aus der Diskussion auszusteigen:

Mit ihrem Redebeitrag (Zl. 197 - 199) in der vorherigen Szene schließt C ihre Argumentation zum Aufgabenteil c) ab. Sie befindet sich in inrem inneren Konflikt, den sie für unlösbar hält, der sie vielleicht auch Frust verspüren lässt. Darin mögen Gründe liegen, dass sie in Zeile 206 nicht Willens ist, K's neuen Gedankenansatz über Tacho meter zu verfolgen. Sie C will weiter- $\quad$ fordert auf (ZI. 206): „Weitermachen“. Damit könnte sie K einerseits animiemachen ren, die Überlegung „Moment, Tausendstel, kleiner Moment“ im Sinne eines Grenzprozesses fortzuführen. Wahrscheinlich will sie aber die Bearbeitung der Teilaufgabe beenden und die nächste beginnen. $\mathrm{K}$ scheint darüber erstaunt zu sein (Zl. 207) „Ja?“ und lehnt ab (Zl. 207): „Wart erst mal." Sie will ihren Gedanken weiterführen und erhofft sich vom Wechsel vom Fahrrad- zum Autotacho Klärung (Zl. 207): „Ist logisch, wenn'st Auto fährst." Diese Formulierung nutzt $C$ für einen weiteren Versuch, aus den Überlegungen zu dieser Teilaufgabe auszubrechen (ZI. 208 - 210), indem sie $\mathrm{K}$ einen bayrischen Dialekt unterstellt. Doch davon lässt sich $\mathrm{K}$ nicht beeindrucken (ZI. 211 - 217).

\section{Szene 4; Zeilen 218 - 247}

218 C: (unterbricht K) Hör mal, wenn da, wenn da eine Momentangeschwindigkeit ist, muss er 219 da immer ja gleich fahren. ... Oder nicht?

$220 \mathrm{~K}$ : Ja, in ner Sekunde. ... Ja, sagen wir mal, wenn (verhaspelt) es in ner Sekunde ist. Aber 221 wenn's jetzt genau $16 \mathrm{Uhr}$ ist? ... (Pause 6s) ... 
222 C: Ja, du, du, ich find, du kannst nur die Durchschnittsgeschwin, ich find (haspeln) ich habe 223 keine Ahnung, aber ich glaub, du kannst nur die Durchschnittsgeschwindigkeit 224 berechnen, weil, äh.

225 K: Berechnen ja.

226 C: Ja oder so.

227 K: Das hätt ich jetzt auch gesagt: Berechnen ja. ... Dass man immer die Durchschnittsge228 schwindigkeit in der Zeit angibt. ... Aber ob es ne Momentangeschwindigkeit gibt..

229 C: (fällt $K$ ins Wort) Also er wird eine Geschwindigkeit meinetwegen um genau 16 Uhr 230 haben. ... ehm

231 K: Ja. (murmelt) Ja ich könnt mir das gut vorstellen, dass es so was gibt. ... (C: Hm) ... 232 (unverständlich, vielleicht) Müssen zu Konsens kommen. ... Aber meines Erachtens, weiß 233 ich nicht, wenn ich mir das so mit nem Auto vorstelle, irgendwo muss du eine 234 Geschwindigkeit haben, in einer bestimmten Zeit. ... (C: Ja, ehm) Du bist ja, ich sag mal, 235 du bist in Bewegung, ... dann musst du die Geschwindigkeit irgendwo ausdrücken 236 können. Oder ob man das so drücken tut, das da ist ehm..

237 C: Drücken tun.. (K lacht)

238 K: Ehmmm, Durchschnittsgeschwindigkeit aus, aber, ... und errechnen kann man auch die 239 Durchschnittsgeschwindigkeit, aber ... Momentangeschwindigkeit ... (Pause 15s)

240 C: Also ich könnte jetzt, ehm, keine genaue Antwort geben...

241 K: Wir könn ja jetzt um den heißen Brei, also so drum herum reden. (lacht) Es könnte so 242 was geben, muss es aber nicht. ... Also für mich wäre es plausibel, wenn es so was 243 geben würde. ... Allein schon weil, wenn man sich das vorstellt, man kann ja nicht nur Durchschnittsgeschwindigkeit fahren. Wenn man jetzt, man kann ... den kleinsten Weg nehmen. Das ist ja auch Durchschnittsgeschwindigkeit.

In der Betrachtung dieser Szene soll weiter verfolgt werden, wie die Schülerinnen mit dem Grundparadoxon weiter umgehen, wie sich der innere Konflikt weiter entwickelt und wie K mit der Frage der Berechnung der Momentangeschwindigkeit umgeht. Doch zunächst soll aufgedeckt werden, welche Art von Geschwindigkeit den Schülerinnen als die grundlegendere erscheint. 
Die konstante Geschwindigkeit; das Problem der Vorstellung lokaler Änderungs-

raten;

r-Welt

Zu den Arten von Geschwindigkeit

C unterbricht $K$ bei ihren Überlegungen zum Tachometer (ZI. 218 - 219):

"Hör mal, wenn da, wenn da eine Momentangeschwindigkeit ist, muss er da immer ja gleich fahren." Wahrscheinlich kann sich $\mathrm{C}$ eine positive lokale Geschwindigkeitsänderungsrate ebenso wenig vorstellen, wie sie sich eine positive lokale Ortsänderungsrate vorstellen kann. Daher ist sie in ihrer Vorstellung auf kleine Zeiträume angewiesen. Bei diesem Gedankengang geht $C$ von einer Momentangeschwindigkeit aus. Da sie keine Information über die Geschwindigkeit in dem kleinen Zeitraum um den betrachteten Zeitpunkt hat, aber kleine Zeiträume für ihre Vorstellung braucht, bleibt ihr nichts anderes übrig, als die bekannte Momentangeschwindigkeit für den ganzen betrachteten kleinen Zeitraum anzunehmen, also eine konstante Geschwindigkeit. In ihrer Vorstellung hat ein Radfahrer in allen kleinen Zeiträumen eine konstante Geschwindigkeit, so dass die Durchschnittsgeschwindigkeiten den Momentangeschwindigkeiten entsprechen. Betrachtet C aber kleine Zeiträume mit den zugehörigen Durchschnittsgeschwindigkeiten, müsste sie diese Zeiträume anschließend zusammensetzen, um an eine Beschreibung der Bewegung des Radfahrers über die gesamte Tour zu kommen. Damit es an den "Nahtstellen" nicht zu Sprüngen kommt, müssen alle Durchschnittsgeschwindigkeiten den gleichen Wert haben. Denn solche Sprünge verbietet die r-Welt-Vorstellung einer Radtour. Daher sieht $C$ sich gezwungen, auf eine konstante Geschwindigkeit über die ganze Radtour zu schließen.

konstante Geschwindigkeit K stimmt C's These "..., muss er da immer ja gleich fahren." zu, schränkt und Zeitpunkte in der r-Welt aber ein (ZI. 220 - 221): "Ja, in ner Sekunde. ... [...] ... Aber wenn's jetzt genau 16 Uhr ist?" Das kann wie folgt interpretiert werden:

1. Damit der Radfahrer überhaupt eine Geschwindigkeit haben kann, muss er in Bewegung sein. Bewegung findet aber in einem Zeitraum, hier einer Sekunde, statt. Aber bewegt er sich auch um genau $16 \mathrm{Uhr}$ ?

2. Es könnte sein, dass sie ihre Gedanken zu den Tachometern weiterführt. Für sie zeigt ein Tachometer immer nur Durchschnittsgeschwindigkeiten für gewisse feste Ortsänderungen oder feste kleine Zeiträume an. Spätestens bei den Tachoüberlegungen ist ihr die Wichtigkeit des 
Unterschieds zwischen Zeitpunkten und kleinen Zeiträumen klar geworden. In diesem Fall könne ihre Antwort bedeuten: Für eine Sekunde gibt es eine Art konstante Durchschnittsgeschwindigkeit, nämlich die, die ein Tacho anzeigt. Aber was passiert an einem Zeitpunkt wie $16 \mathrm{Uhr}$ ?

3. Andererseits könnten ihre ausgeprägten $\mathrm{rWelt-Vorstellungen} \mathrm{ihre} \mathrm{Re-}$ aktion hervorrufen. Erfahrungsgemäß fährt man auf einer Radtour nicht mit einer konstanten Geschwindigkeit. Für eine Sekunde der Radtour kann das schon mal vorkommen. Hier könnte eine Altagserfahrung eine Rolle zu spielen, dass ein Radfahrer in einer Sekunde kaum eine wesentliche Beschleunigung erzielen kann. (K geht nicht davon aus, dass der Radfahrer gegen einen Baum fährt.) Auch hier bleibt aber unklar, ob zu einem Zeitpunkt eine Momentangeschwindigkeit vorliegen kann.

die konstante Geschwindigkeit ist grundlegend
In dem Versuch, C's Gedankengang nachzuzeichnen, taucht ein dritter Typ von Geschwindigkeit auf, die konstante Geschwindigkeit. Den Gedankengängen meine ich entnehmen zu können, dass die konstante Geschwindigkeit als grundlegend angesehen wird: Die Überlegungen beginnen mit der Momentangeschwindigkeit (ZI. 218) „..., wenn da eine Momentangeschwindigkeit ist,“. Wie gezeigt, wird dann versucht, die Momentangeschwindigkeit (auf kleinen Zeiträumen) mit Hilfe der konstanten Geschwindigkeit zu erklären. (Zl. 219) ,..., muss er ja immer gleich fahren.“ Da C in ihren Vorstellungen einen Zeitraum benötigt, braucht sie auch die konstante Geschwindigkeit, um sich Momentangeschwindigkeit erklären zu können.

Obwohl die Momentangeschwindigkeit diejenige ist, die den Mädchen das Kopfzerbrechen bereitet, scheint sie in diesen Überlegungen doch der Ausgangspunkt zu sein und daher grundlegender als die Durchschnittsgeschwindigkeit. Wichtig erscheint mir hier, dass die genannten Gedankengänge im Wesentlichen die r-Welt bemühen. Eine weitere Bestätigung dieser "Rangordnung“ der Geschwindigkeiten sehe ich in K's folgendem Redebeitrag am Ende dieser Szene (Zl. 245 - 246): „Allein schon weil, wenn man sich das vorstellt, man kann ja nicht nur Durchschnittsgeschwindigkeit 
Fazit: lokale Änderungsraten

fahren." Also muss man immer eine Momentangeschwindigkeit fahren, damit eine Durchschnittsgeschwindigkeit irgendwie zustande kommen kann. Die Durchschnittsgeschwindigkeit könnte als verallgemeinerter Mittelwert im Sinne der Integralrechnung gesehen werden.

C begegnet dem Problem der lokalen Änderungsrate in zwei verschiedenen Gestalten: lokale Weg- und lokale Geschwindigkeitsänderungsrate. In beiden Fällen bereitet ihr die Vorstellung der positiven lokalen Änderungsrate Probleme. Vermutlich kann sie sich lokale Änderungsraten an sich nicht vorstellen. Das würde bedeuten, dass ihr die Grundvorstellung „lokale Änderungsrate" vom Ableiten an sich Probleme bereitet, unabhängig vom Kontext (Momentangeschwindigkeit, Beschleunigung, Tangentensteigung, ...), in den sie eingebettet ist. Die Grundvorstellung, die Schülerinnen und Schülern eine Hilfe bei der Begriffsbildung sein soll, ist vermutlich ihr Problem.

\section{Zu den Argumentationsmustern}

Bis hier folgert $\mathrm{K}$ aus ihren $\mathrm{r}$-Welt-Vorstellungen, die die einbettende Sichtweise bemühen, dass es eine Momentangeschwindigkeit gibt. $C$ hat das Ziel, die Momentangeschwindigkeit zu berechnen, wobei sie $\mathrm{r}$ - und $\mathrm{m}$-WeltVorstellungen in der isolierenden Sichtweise heranzieht. Erstaunlicherweise scheinen beide in dieser Szene die Meinung zu ändern und jeweils die Position der anderen einzunehmen:

$\mathrm{C}$ und $\mathrm{K}$ ändern scheinbar ihre anfangs vertretene Meinung
C leitet diesen Vorgang mit einer längst bekannten Aussage ein (ZI. 222 224): „Ja, du, du, ich find, du kannst nur die Durchschnittsgeschwin, ich find (haspeln) ich habe keine Ahnung, aber ich glaub, du kannst nur die Durchschnittsgeschwindigkeit berechnen, weil, äh." Dennoch scheint für sie in dieser Aussage eine neue Erkenntnis vorzuliegen, da sie vielleicht vor Aufregung etwas stottert und sich verhaspelt. $\mathrm{K}$ scheint sich jetzt so weit auf das Berechnungsproblem eingelassen zu haben, dass sie diese Äußerung bestätigen kann (Zl. 227): „Das hätt ich jetzt auch gesagt: Berechnen ja." Dann kommt der Seitenwechsel: Bis hier hatte sich $\mathrm{K}$ mit einer 
für sie logischen Argumentation die Existenz der Momentangeschwindigkeit gesichert. Nun fragt sie (ZI. 228 - 229): „Aber ob es ne Momentangeschwindigkeit gibt.." Und auch C, die bisher die Existenz der Momentangeschwindigkeit abgelehnt hat, ändert nun ihre Meinung, wenn sie direkt auf K's Frage entgegnet (ZI. 230 - 231): „Also wird er eine Geschwindigkeit meinetwegen um genau $16 \mathrm{Uhr}$ haben." Dem stimmt $\mathrm{K}$ jetzt wieder zu (Zl. 232): „Ja ich könnt mir das gut vorstellen, dass es so was gibt.“

Wahrscheinlich wechseln beide nicht wirklich ihre Meinungen. Oben wurde schon vom inneren Konflikt, dem Grundparadoxon berichtet. Die einzelnen Äußerungen spiegeln dann nur ein Hin- und Herpendeln zwischen den beiden Positionen wieder. Dass sich die Mädchen beider Positionen bewusst sind, wird an der schriftlichen Bearbeitung der Aufgabe deutlich, in der beide Positionen getrennt wiedergegeben werden, vgl. Abbildung 9. Auch die Fortsetzung von K's Redebeitrag zeigt das (ZI. 233): „Müssen zu einem Konsens kommen."

Dann führt $\mathrm{K}$ noch einmal die beiden Positionen für sich an: Die $\mathrm{r}$-WeltVorstellung einer Autofahrt bekräftigt die Existenz der Momentangeschwindigkeit, aber ob man die „ausdrücken kann“ (vgl. Zl. 237). Wahrscheinlich bedeutet „ausdrücken können“ für sie, dass man eine Zahl angeben können muss, die den Wert der Momentangeschwindigkeit wiedergibt. Diese Zahl könnte berechnet oder gemessen werden, wie die vorangegangenen Überlegungen zeigten. Ähnliche Überlegungen wie in den Zeilen 232 bis 237 führt $\mathrm{K}$ noch einmal in den Zeilen 243 bis 247 an. $\mathrm{m}$ letztgenannten Absatz macht sie sich über „kleinste Wege“ (Zl. 247) Gedanken. Diese Gedanken werden in der folgenden Szene wieder aufgenommen und dort analysiert.

$\mathrm{K}$ stellt eine Forderung an die Mathematik
Interessant scheint mir hier noch K's Formulierung zu sein (ZI. 236 - 237): Man muss die Momentangeschwindigkeit ausdrücken können. Nachdem sie zuvor die Existenz der Momentangeschwindigkeit mit r-Welt-Überlegungen gesichert hat, fordert sie nun von der Mathematik (im Fall, dass sie die Momentangeschwindigkeit berechnen will) oder der Physik (im Fall, dass 
sie die Momentangeschwindigkeit messen will und ein Messvorgang der Physik zugeschrieben wird) eine Möglichkeit, den Wert der Momentangeschwindigkeit zu bestimmen. Diese Forderung zeigt an, dass sie die Erfahrungswelt über die Mathematik- oder Physik-Welt stellt. Die letztgenannten haben also die Aufgabe, die Erlebniswelt zu beschreiben oder dieser zu dienen. Diese Einstellung ins besondere zur Mathematik ist nicht allgemein verbreitet, wie andere Transkripte zeigen. Dort wird schon mal die m-Welt über die r-Welt gestellt, wenn aus Überlegungen in der m-Welt hervorgeht, dass die Momentangeschwindigkeit nur approximativ berechenbar ist, es sie daher in der r-Welt nicht gibt.

\section{C's zweiter Ausbruchsversuch}

In der vorherigen Szene hat $\mathrm{C}$ durch ihren Ausbruchsversuch gezeigt, dass sie aus ihrem Widerspruch nicht herauskommt und daher keine Lust mehr hat, die Teilaufgabe zu bearbeiten und nun eine neue Teilaufgabe beginnen will. Trotz des Widerspruchs entwickelt sie zu Beginn dieser Szene neue Gedanken (ZI. 218 - 219; „Er muss immer gleich fahren.“) und schöpft daraus vielleicht Hoffnung, doch noch weiter kommen zu können, wie ihre Aufregung in ZI. 222 zeigt. Doch bereits in Zl. 224 erfährt sie eine Ernüchterung. Sie zieht sich etwas zurück, wie der zweite Teil der Szene zeigt, in dem sie von 21 Dialogzeilen nur einen Redeanteil von 4 Zeilen hat. Zudem kann man einen weiteren Ausbruchsversuch erkennen: $\mathrm{K}$ formuliert etwas ungeschickt (ZI. 237) „Ob man das so drücken tut,“. Analog zu der „Kommst aus Bayern“-Szene versucht C daraus einen kleinen Witz zu machen (ZI. 238): „Drücken tun..“ worauf K auch lacht, aber dann ihre Gedanken weiter führt.

\section{Szene 5; Zeilen 248 - 272}

C: (fällt $K$ ins Wort) Ja das muss ja auch ehm ... Ja, ja, das ja nen Bruch, das muss genau um 16 Uhr sein. Das ist.. durch gefahren ist. ... Haben 
252 C: Ja eben ja noch nicht mal. Weil dann hast ja (K: Ja.) schon wieder 2 Sekunden, die Zeit 253 läuft doch weiter. ... (K: Ja.) Die bleibt ja nicht stehen. (K lacht) Die kann ja nicht genau 254 um 16 Uhr...

255 K: Den Punkt haben. ...

256 C: Ach, schreib was hin. Also ich würd sagen, nein, aber hm ... (Pause 8s) ...

257 K: Ich weiß es auch nicht, (unverständlich)

258 C: Weil du kannst doch die Geschwindigkeit nicht messen, wenn du keine Zeit hast. ... Und 259 momentan ist ja um eine bestimmte Zeit, und das muss doch genau an diesem Punkt 260 sein. ... Aber du brauchst ja auch, ehm, wenn ein Weg zurücklegst, die Zeit. Und das 261 wäre ja dann, wenn's ein Zeitraum ist durchschnittlich. Er fährt meinetwegen in ner 262 Sekunde durchschnittlich so und so weit. ... (K: Man könnt ja..) Wenn du keine Zeit hast, 263 wenn (K: Unendlich kleinste Einheit.) du genau 16 Uhr hast, wenn du da ehm die 264 Geschwindigkeit messen willst, hast du doch keine Zeit, weil das ist genau 16 Uhr, und 265 du darfst ja nicht weiter rechnen, weil du sonst wieder ne Durchschnittsgeschwindigkeit 266 hast. ...

$267 \mathrm{~K}:$ Ja, irgendwie ist das auch logisch. ... Ach, das ist, ich weiß es nicht, warum ...

268 C: Ganz am Anfang hab ich auch gedacht, das gibt es nicht. (Durcheinander)

269 K: Ganz spontan hätt ich gesagt, es gibt so was, aber wenn man ehrlich (???) ...

270 (25:40) C macht den Vorschlag, beide Meinungen jeweils mit Begründung aufzuschreiben. 271 Zunächst wird die „Es gibt eine Momentangeschwindigkeit“-Antwort geschrieben. K schreibt 272 und lässt die Antwort von C kontrollieren. (27:20)

\section{Zusammenhang von Ort und Zeit}

kleine und kleinste Größen in der m-Welt
Die vorherige Szene endete damit, dass $\mathrm{K}$ sich einen Bewegungsvorgang vorstellte ( $r$-Welt) und überlegte, dass auch zu dem kleinsten Weg eine Durchschnittsgeschwindigkeit gehört (ZI. 245 - 247). Diese Szene beginnt damit, dass C einen Bogen zur m-Welt schlägt (Zl. 248): „Ja, ja, das ja nen Bruch, das muss genau um 16 Uhr sein." Vielleicht hat sie sich von K's kleinsten Wegen anregen lassen, wieder über die Bedeutung des Zeitpunkts 16 Uhr Gedanken zu machen. K versucht nun, mit Hilfe der Vorstellung der Radfahrsituation die Ortsänderung zu ermitteln, damit $C$ rechnen kann (ZI. 250 - 251): „Ja, das muss genau wissen wie... Wie viel Zentimeter 
eh man da durch gefahren ist." Damit ist C nicht zufrieden (ZI. 252): „Ja eben nicht mal." Sie ist sich der Bedeutung des Zeitpunkts nun wirklich bewusst geworden. Schon in K's Redeabschnitt hat sie eingeworfen (ZI. 150) „Genau um 16 Uhr“. Sie begründet ihre Unzufriedenheit so (Zl. 252 254): Zentimeter sind nicht zugelassen, „weil dann hast ja (K: Ja.) schon wieder 2 Sekunden, die Zeit läuft doch weiter. ... (K: Ja.) Die bleibt ja nicht stehen. (K lacht.) Die kann ja nicht genau um 16 Uhr..." Das heißt, dass auch die kleinste Ortsänderung nur in einem Zeitraum ablaufen kann.

$\mathrm{K}$ bestätigt jeden Absatz der Argumentation. Sie scheint $\mathrm{C}$ gut folgen zu können. An einer Stelle lacht sie sogar. Vielleicht ist das die Stelle, an der ihr klar wird, dass „kleine Wege“ hier nicht weiterhelfen. In dem Fall wäre das Lachen eines, das vielleicht etwas Resignation anzeigt, nach dem Motto: Du hast ja recht.

$\mathrm{C}$ bringt sich und $\mathrm{K}$ durch ihre Argumentation an einen Punkt der Resignation (Zl. 256 - 257): C: „Ach, schreib was hin. Also ich würd sagen, nein, aber hm... K: Ich weiß es auch nicht." In den vorhergehenden Szenen habe ich versucht, C's inneren Konflikt zu beschreiben. Dabei habe ich ihr das 0/0-Problem zugeschrieben, aus dem sie die Nichtexistenz der Momentangeschwindigkeit schloss. Dabei habe ich auch auf die schriftliche Bearbeitung hingewiesen. In C's folgendem Redebeitrag stellt sie erstmals explizit den Zusammenhang zwischen einer Ortsänderung der Größe Null zu einem Zeitpunkt (der Länge Null) her, der das 0/0-Problem offenbar werden lässt (ZI. 258 - 266):

das 0/0-Problem explizit; Was kann man messen? unendlich kleinste Größen
„Weil du kannst doch die Geschwindigkeit nicht messen, wenn du keine Zeit hast. ... Und momentan ist ja um eine bestimmte Zeit, und das muss doch genau an diesem Punkt sein. ... Aber du brauchst ja auch, ehm, wenn ein Weg zurücklegst, die Zeit. Und das wäre ja dann, wenn's ein Zeitraum ist, durchschnittlich. Er fährt meinetwegen in ner Sekunde durchschnittlich so und so weit. ... (K: Man könnt ja..) Wenn du keine Zeit hast, wenn (K: Unendlich kleinste Einheit.) du genau 16 Uhr hast, wenn du da ehm die Geschwindigkeit messen willst, hast du doch keine Zeit, weil das ist genau 
Wann kann man 16 Uhr, und du darfst ja nicht weiter rechnen, weil du sonst wieder ne rechnen?

$\mathrm{r}$ - und $\mathrm{m}$-Welt Durchschnittsgeschwindigkeit hast. ..." Das Messen dient der Ermittlung des Wertes der Ortsänderung. Die Betrachtung eines Zeitpunkts unterbindet die Messung, ein Ort (Punkt) kann nicht gemessen werden.

Noch einmal das Grundparadoxon in den Sicht weisen

$\mathrm{K}$ versucht logisch zu denken und ist für Argumente zugänglich. In Szene 3 sagt sie, dass sie ihre Argumentation (ein bewegter Körper hat zu jedem Zeitpunkt der Bewegung eine Momentangeschwindigkeit) plausibel findet (ZI. 244). C's Argumentation hier gesteht sie auch eine Logik zu (Zl. 267): „Ja, irgendwie ist das auch logisch." Schon während $\mathrm{C}$ argumentiert hat $\mathrm{K}$ das Problem verstanden, denn sie versucht, ihren Gedanken der „kleinsten Wege“ zu verbessern (ZI. 262 - 263): „Man könnt ja.. Unendlich kleinste Einheit." Hier erfindet sie für sich ein Objekt aus der Welt des unendlich Kleinen. Vermutlich schreibt sie ihrem Objekt „unendlich kleinste Einheit“ folgende Eigenschaften und Bedeutungen zu: Für die Berechnung braucht man Einheiten, daher die Wortwahl „Einheit“. Die Größe der Einheit muss aber so klein sein, dass man mit ihr eine Momentangeschwindigkeit und nicht nur eine Durchschnittsgeschwindigkeit ermitteln kann. Andererseits muss sie groß genug sein, damit man überhaupt mit ihr rechnen kann.

Beide stehen nun in einem inneren Konflikt, der in den Zeilen 268 und 269 zum Aus druck kommt:

C: Ganz am Anfang hab ich auch gedacht, das gibt es nicht. (Durcheinander)

K: Ganz spontan hätt ich gesagt, es gibt so was, aber wenn man ehrlich (???) ...

der naive Geschwindigkeitsbegriff wird in Frage gestellt
Bezeichnend ist, dass beide ihre eingangs eingenommene Position jeweils in Frage stellen. Es scheint, als habe die Bearbeitung des Aufgabenteils $\mathrm{c}$ ) bei den Mädchen zu einem Prozess der Bedeutungsveränderung des Begriffs Momentangeschwindigkeit geführt. (ZI. 269) „Ganz spontan hätt ich gesagt, es gibt so was, aber wenn man ehrlich (???)..." Die Spontaneität am Anfang bringt einen Begriff der Momentangeschwindigkeit an den Tag, der vielleicht naiven Vorstellungen der Alltagswelt entspringt. Die intensive 
Aus einandersetzung mit diesem Begriff führt zu einem Prozess der Bedeutungsänderung, wobei die Naivität in Frage gestellt wird (,....aber wenn man ehrlich (???)...").

Am Ende dieser Szene verfügen die Schülerinnen über zwei in sich logische Argumentationen, die zu verschiedenen Antworten bzgl. der Frage nach der Existenz der Momentangeschwindigkeit führen. Es wurde versucht, das Auftreten des Grundparadoxons mit Hilfe Sichtweisen aufzudecken. Für diese beiden Schülerinnen hat sich gezeigt, dass die isolierende Sichtweise sowohl in der $\mathrm{k}$ also auch m-Welt eingenommen werden konnte, die einbettende nur in r-Welt-Vorstellungen anzutreffen war. 


\subsection{Transkript 14}

$\begin{array}{ll}\text { Datum } & : 9.6 .1999,15: 30-16: 33 \\ \text { Ort } & : \text { Pelizaeus Gymnasium, A308 }\end{array}$

Teilnehmer : Claudia B. (links im Bild) und David K. (rechts im Bild); Jahrgangsstufe 12 des Pelizaeus Gymnasiums, beide LK

Aus diesem Transkript sollen nur zwei Szenen vorgestellt werden. Die erste Szene gibt wieder, wie C und D den Aufgabenteil c) bearbeiten. Die zweite Szene zeigt einen Ausschnitt des Gesprächs, das der Versuchsleiter im Anschluss an die Partnerarbeitsphase mit C und D geführt hat. In diesem Ausschnitt greift der Versuchsleiter die schriftliche Lösung zu Aufgabenteil c) noch einmal auf.

Zunächst wird beschrieben, wie C und D die Aufgabenteile a) und b) bearbeiten.

\section{Zum Aufgabenteil a) und b)}

Beide lesen sich den Aufgabentext zu Aufgabenteil a) durch und überlegen schweigend. Nach 40 Sekunden macht $D$ den Vorschlag, einen in der Tabelle gegebenen Wert zu überprüfen. C wertet mit dem Taschenrechner die Funktion an einer Stelle aus. Die Überprüfung war erfolgreich, daher wird nun $\mathrm{f}(3)$ berechnet.

Dann wird Aufgabenteil b) aufgedeckt. Wieder lesen beide 40 Sekunden lang den Aufgabentext. Dann wird Blatt a) noch einmal herangezogen. D hat die Idee „Weg durch Zeit“ zu berechnen. Er fordert C auf, 117.6 durch 3 zu teilen. Im Anschluss werden auch die Durchschnittsgeschwindigkeiten der jeweils ersten x Stunden berechnet. Auch der in der Wertetabelle fehlende Funktionswert wird ermittelt. Dann erst fält $D$ auf, dass sie fälschlich die Durchschnittsgeschwindigkeit der ersten $x$ Stunden berechneten. C will diesen Einwand übergehen, aber der Versuchsleiter ermuntert, darüber nachzudenken. Nun will D die Durchschnittsgeschwindigkeit für jede einzelne Stunde berechnen. $C$ ist nicht einverstanden, hat aber zunächst keinen besseren Vorschlag. Dann sagt sie etwas mit „Abziehen“, worauf D die Berechnung der Wegdifferenz erläutert. Nun werden die richtigen 
Berechnungen durchgeführt und die Ergebnisse notiert. Nur beim Wert für die letzten $1 \frac{1}{2}$ Stunden haben sie sich verrechnet.

\section{Szene 1; Zeilen 25 - 45}

Nach ca. 13 Minuten Gesamtarbeitszeit decken sie Aufgabenteil c) auf. Ungefähr 30 Sekunden lang lesen sie die Aufgabe und denken nach.

25 D: Also, natürlich hat er ne Geschwindigkeit drauf, aber die können wir nicht bestim26 men. ... (Pause 10s) ... Ja, würd ich auch so aufschreiben. ... Also... oder was 27 meinst Du?

28 C: Weiß nicht. ... (Pause 9s) ... nein, eigentlich...

29 D: Also, ich würd sagen, eine Momentangeschwindigkeit gibt es ja immer. Also, 30 wenn du Fahrrad fährst, hast du ja immer ne Momentangeschwindigkeit, aber in 31 diesem Fall können wir die ja nicht ausrechnen. Aber das gibt es ja trotzdem. Also 32 würd ich sagen: natürlich gibt es eine Momentangeschwindigkeit, nur lässt sie sich 33 mit diesen Werten nicht berechnen.

34 C: Na gut. (beide lachen, $C$ schreibt das auf)

35 D: Berechnen können wir es ja nicht, ne. Er konnte ja vielleicht... . Am Anfang konnte 36 er ja vielleicht mal, wir haben ja bisher nur Durchschnittsgeschwindigkeit berech37 net. Es kann ja sein, dass er die ersten zwei Stunden, was weiß ich, 60 gefahren 38 ist und dann nochmals 20 getrottet hat.

39 C: Hm! ... Wir haben ja nur die Formel für einen Zeitraum ... zu einem Zeitpunkt. ... 40 (D: Hm! Pause 5s) ... nicht für einen Zeitpunkt...

41 D: Nach der Funktion lässt sie sich bestimmt nicht ... berechnen, zeigt nur an wie 42 weit er gekommen ist.

43 C: Ja ... (Pause 6 s; beide beschließen, den zweiten Teil der Bearbeitung aufzu44 schreiben. C schreibt und murmelt dabei) ... Müssen wir bestimmt gleich berech45 nen. (Beide lachen)

In dieser Szene hat C nur einen geringen Redeanteil. Daher soll hier nur D's Argumentation aufgezeigt werden. 
D's einbettende Sicht in der $r$ Welt

\section{D's Argumente}

Zunächst trifft D zwei Aussagen, ohne sie zu begründen (ZI. 25 - 26): „Also, natürlich hat er ne Geschwindigkeit drauf, aber die können wir nicht bestimmen." Zudem ist nicht klar, ob D mit dem Wort „Geschwindigkeit“ die Durchschnittsgeschwindigkeit oder die Momentangeschwindigkeit meint. Sein nächster Redeabschnitt, in dem er auch eine Begründung für die Existenz der Momentangeschwindigkeit anführt, zeigt, dass er die Momentangeschwindigkeit meint (ZI. 29 - 30): „Also, ich würd sagen, eine Momentangeschwindigkeit gibt es ja immer. Also, wenn du Fahrrad fährst, hast du ja immer ne Momentangeschwindigkeit,..... D stellt sich vor, wie man Rad ährt. Das Wort „immer“ zeigt an, dass er sich einen Zeitraum vorstellt, in dem man immer Momentangeschwindigkeit hat. Weil er sich so einen Abschnitt der Radtour vorstellt, kann er sich auch die Bewegung des Radfahrers in diesem Zeitraum vorstellen. Es zeigt sich, dass $D$ in der $r$-Welt eine ausgeprägte einbettende Sichtweise an den Tag legt.

Für D gibt es Momentangeschwindigkeit, obwohl man diese nicht berechnen kann
Im Anschluss an diese Argumentation bekräftigt $D$ noch einmal, dass er die Momentangeschwindigkeit nicht berechnen kann (ZI. 30 - 31): ,....aber in diesem Fall können wir die ja nicht ausrechnen." Zum zweiten Mal betont $\mathrm{D}$, dass er bzw. $\mathrm{C}$ und er die Momentangeschwindigkeit nicht berechnen können (vgl. Zl. 25). Es scheint für ihn kein generelles Problem vorzuliegen. Als Bestätigung dieser Interpretation kann auch die Formulierung „in diesem Fall“ gesehen werden. Erst nachdem D noch einmal die Existenz der Momentangeschwindigkeit bekräftigt hat (ZI. 31 - 32) „Aber das gibt es ja trotzdem. [...] natürlich gibt es eine Momentangeschwindigkeit“ begründet er, worin in diesem Fall das Problem liegt, nämlich dass die Momentangeschwindigkeit nicht berechenbar ist (ZI. 32 - 33): ,....nur lässt sie sich mit diesen Werten nicht berechnen." Mit „diesen Werten" meint D wahrscheinlich die in der Wertetabelle gegebenen. Auf der Videoaufnahme kann man erkennen, dass direkt vor inm das Blatt a) liegt, er deutet jedoch nicht darauf. Andererseits könnte er auch die Werte meinen, die mit Hilfe der Funktionsgleichung berechenbar sind. Auch die Funktionsgleichung ist auf Blatt a) abgebildet. In keinem Fall wird an 
dieser Argumentation deutlich, warum die gegebenen Werte für $D$ die Berechnung nicht ermöglichen. Die Begründung dafür gibt $D$ in seinem nächsten Redebeitrag (ZI. 36 - 38):

D unterscheidet Durchschnittsgeschwindigkeit und Momentangeschwindigkeit
D kann Momentangeschwindigkeit trotz einbettender Sichtweise nicht berechnen

D hat das $0 / 0$ Problem nicht „....wir haben ja bisher nur Durchschnittsgeschwindigkeit berechnet. Es kann ja sein, dass er die ersten zwei Stunden, was weiß ich, 60 gefahren ist und dann nochmals 20 getrottet hat." - Bis hier konnte man nur annehmen, dass $D$ einen Unterschied zwischen Durchschnittsgeschwindigkeit und Momentangeschwindigkeit sieht. Hier wird diese Annahme bestätigt, denn $D$ sagt (sinngemäß): ,Bisher haben wir nur die Durchschnittsgeschwindigkeit berechnet. Jetzt soll etwas anderes getan werden." Dann vergleicht $D$ Werte, die 60 (für die ersten zwei Stunden) und die 20 (für die dritte Stunde). Es wird nicht deutlich, ob die Zahlen zurückgelegte Weglängen oder Durchschnittsgeschwindigkeiten der jeweiligen Zeitabschnitte sein sollen. Da D zuvor von Durchschnittsgeschwindigkeiten gesprochen hat, ist anzunehmen, dass er auch jetzt Durchschnittsgeschwindigkeiten meint. Auf den Punkt gebracht, bedeutet D's Argumentation:

14 Uhr beginnt die Radtour. Nach zwei Stunden Fahrt ist man an dem in Aufgabenteil c) zu betrachtenden Zeitpunkt 16 Uhr angelangt. Man kann mit den Werten der Wertetabelle die Momentangeschwindigkeit um 16 Uhr nicht berechnen, weil die Durchschnittsgeschwindigkeiten der benachbarten Zeiträume 14 bis $16 \mathrm{Uhr}$ und 16 bis $17 \mathrm{Uhr}$ verschieden sein können. An dem Zeitpunkt 16 Uhr zwischen den beiden Zeiträumen kann also ein Sprung der Durchschnittsgeschwindigkeitswerte entstehen. Man kann also keine Aussage über die Momentangeschwindigkeit um $16 \mathrm{Uhr}$ treffen.

Interessant erscheint hier, dass D im Vergleich zu anderen Schülerinnen und Schülern nicht die isolierende Sichtweise mit Zeitpunkt-Betonung einnehmen muss, um die Nicht-Berechenbarkeit zu zeigen. Zwar überlegt auch er, was zum Zeitpunkt 16 Uhr passiert. Dabei behält er aber die Radtour über die ersten drei Stunden als Ganzes im Auge. Er argumentiert insbesondere nicht über das 0/0-Problem, dass also zu einem 
Zeitpunkt (Nenner $=0$ ) keine Ortsänderung stattfinden kann $($ Zähler $=0$ ).

D's Problem liegt eher darin, dass die Wertetabelle nur Werte für jede halbe Stunde liefert. Das könnte er mit seinen Worten „in diesem Fall“ gemeint haben. Da die Wertetabelle Ursache für die Beschränkung der Betrachtung auf Zeiträume zu sein scheint, soll $D$ auch nicht die isolierende Sichtweise mit Zeitraum-Betonung unterstellt werden. Es wird allerdings nicht klar, was für Werte er meint zu benötigen, um die Momentangeschwindigkeit berechnen zu können.

C hat offenbar mitgedacht und überlegt auch, was zur Berechnung der Momentangeschwindigkeit zur Verfügung steht und was benötigt wird (ZI. 39): „Wir haben ja nur die Formel für einen Zeitraum ... zu einem Zeitpunkt." Es ist nicht ganz klar, ob sie mit der Formel die Gleichung Durchschnittsgeschwindigkeit $\mathrm{v}=\Delta \mathrm{S} / \Delta \mathrm{t}$ oder die Gleichung der Weg-Zeit-Funktion meint. Beide Gleichungen geben Werte für Zeiträume (unterschiedlichen Charakters) an: Für die Durchschnittsgeschwindigkeit ist das unmittelbar klar; die Weg-Zeit-Funktion gibt laut Aufgabenteil a) an, wie weit Jan Ullrich bis zu einem Zeitpunkt schon vorangekommen ist. Hier wird also die Zeitspanne vom Start bis zu einem Zeitpunkt betrachtet. Nach wie vor überlegt $\mathrm{D}$, wie er die Momentangeschwindigkeit berechnen könnte. Durch C's Beitrag wird er angeregt, die Funktionsgleichung zu betrachten. Doch das hilft inm nicht weiter (ZI. 40 - 41): „Nach der Funktion lässt sie sich bestimmt nicht ... berechnen, zeigt nur an wie er weit er gekommen ist." Die hier aufgeführten Argumente sind auch so in die schriftliche Bearbeitung eingegangen (vgl. Abbildung 10). Bis zum Ende dieser Szene wurden nur die ersten drei Zeilen geschrieben, die später durchgestrichen wurden. 
c) Bisher war immer von Durchschnittsgeschwindigkeiten die Rede. Gibt es auch so etwas wie eine Momentangeschwindigkeit um 16:00 Uhr? Begründe!

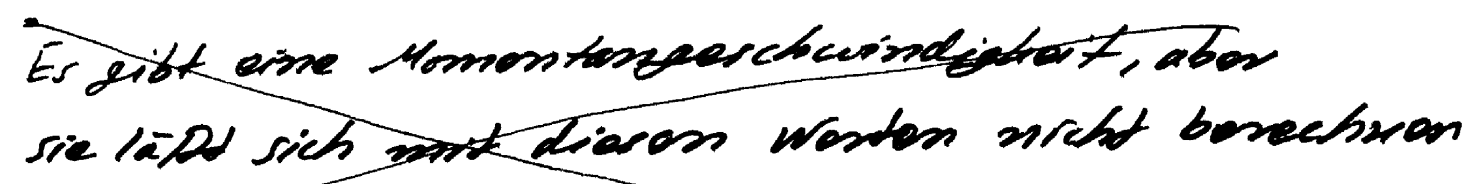

Dre Fn. zeigd lediglich den wurrickgelegten weg an

Wir bestimmon die Darchschnittegerchusndighaid fir hleinore Zeitabschritte und kamme so an einen Wat der an die Mommentan geschwindigkeit möglidst gencen herankernnt. Da der Faher un 16:00 Uhe eine beohiminte Goschuindigkeit haber mupk, gill es and eire Mamartan gesolwridigkeit.

Abbildung 10: Die Bearbeitung von Aufgabenteil c)

\section{Dund die Denkwelten}

Aufgabenteil c) fordert zu einer Entscheidung (Gibt es so etwas wie eine Momentangeschwindigkeit?) und Begründung auf. Betrachtet man die ersten drei großen Redeabschnitte von D, fällt auf, dass er die Entscheidung aufgrund von rWelt-Vorstellungen trifft und den Wunsch nach einer Berechnungsmöglichkeit in der $r$ - oder m-Welt gegenüberstellt. Begründungen dafür, dass er den Wert der Momentangeschwindigkeit nicht berechnen kann, sucht er in beiden Welten. Folgende Tabelle soll das Pendeln zwischen den Welten aufzeigen. Insbesondere das Argument, dass die Momentangeschwindigkeit nicht berechenbar ist, weil der Radfahrer verschieden schnell sein kann, zeigt, dass D versucht, Zusammenhänge zwischen den Welten aufzuzeigen. Der Wunsch nach einer Berechnungsmöglichkeit ist nicht ohne weiteres einer Denkwelt alleine zuzus chreiben. Daher kann man keine seiner Äußerungen jeweils eindeutig einer Denkwelt zuordnen. Das dichte Zusammenspiel der Welten halte ich aber hier für interessant genug, um eine Tabelle mit meinen Einschätzung zu erstellen, welche Welt $D$ an welcher Stelle wohl stärker betonen mag, vgl. Tabelle 3. In der 
linken Spalte befinden sich Aussagen, die ich eher der $\mathrm{kWelt}$ zuordne, in der rechten Spalte die, die ich der mWelt zuordne. Es wird nur dann die Spalte gewechselt, wenn es eine Aussage erfordert. Füllsätze, die sich keiner Welt eindeutig zuordnen lassen, werden der vorherigen Aussage einfach angehängt:

\begin{tabular}{|c|c|c|}
\hline$\overline{\mathrm{Zl}}$. & eher r-Welt & eher m-Welt \\
\hline 26 & $\begin{array}{l}\text { Also, natürlich hat er ne Geschwindig- } \\
\text { keit drauf, }\end{array}$ & $\begin{array}{l}\text { aber die können wir nicht bestimmen. } \\
\text {... (Pause 10s) ... Ja, würd ich auch so } \\
\text { aufschreiben. ... Also ... oder was } \\
\text { meinst Du? }\end{array}$ \\
\hline 30 & $\begin{array}{l}\text { Also, ich würd sagen, eine Momentan- } \\
\text { geschwindigkeit gibt es ja immer. Also, } \\
\text { wenn du Fahrrad fährst, hast du ja }\end{array}$ & \\
\hline 31 & $\begin{array}{l}\text { immer ne Momentangeschwindigkeit, } \\
\text { Aber das gibt es ja trotzdem. Also } \\
\text { würd ich sagen: natürlich gibt es eine }\end{array}$ & $\begin{array}{l}\text { aber in diesem Fall können wir die ja } \\
\text { nicht ausrechnen. }\end{array}$ \\
\hline $\begin{array}{l}33 \\
35\end{array}$ & Momentangeschwindigkeit, & $\begin{array}{l}\text { nur lässt sie sich mit diesen Werten } \\
\text { nicht berechnen. } \\
\text { Berechnen können wir es ja nicht, ne. } \\
\text { Er konnte ja vielleicht... . }\end{array}$ \\
\hline 36 & Am Anfang konnte er ja vielleicht mal, & wir haben ja bisher nur Durchschnitts- \\
\hline 37 & $\begin{array}{l}\text { Es kann ja sein, dass er die ersten } \\
\text { zwei Stunden, was weiß ich, } 60 \\
\text { gefahren ist und dann nochmals } 20 \\
\text { getrottet hat. }\end{array}$ & geschwindigkeit berechnet. \\
\hline
\end{tabular}

Tabelle 3: D und die Denkwelten

Das Pendeln zwischen den Denkwelten selber kann als Indiz angesehen werden, dass $D$ enge Verbindungen zwischen den Welten sieht. Seine Redebeiträge zeigen aber deutlich an, dass es Ungereimtheiten gibt. In der $\mathrm{r}-$ 
Welt gibt es für inn die Momentangeschwindigkeit (ZI. 25, 29, 31, 32), der Berechnungs-Wunsch ist für inn aber weder in $r-$ noch in $m$-Welt erfüllbar (ZI. 25, 31, 33, 35). Die Nicht-Berechenbarkeit war für andere Probanden Grund genug, die Existenz der Momentangeschwindigkeit zu bestreiten. Für D gilt das nicht (ZI. 31): „Aber das gibt es ja trotzdem.“

$D$ ist in dieser Szene der Wortführer

In dieser Szene ist $\mathrm{D}$ der Wortführer. Schnell hat er eine in der $\mathrm{r}$-Welt schlüssige Begründung für die Existenz gefunden. (Ein Körper, der sich bewegt, hat eine Momentangeschwindigkeit.) Dem hat $C$ nichts entgegenzusetzen. Wie die folgende Szene zeigt, hat $D$ sie sogar überzeugt. Vielleicht spricht $D$ auch nur Gedanken aus, die $C$ eh schon hatte, und bekräftigt sie also nur.

Die längeren Pausen im ersten Abschnitt der Szene und D's direktes Nachfragen bei C (vgl. Zl. 27), ob sie mit seinen Äußerungen übereinstimmt, zeigen, dass $D$ zwar Wortführer ist, $C$ aber dennoch Möglichkeiten der Meinungsäußerung gibt.

\section{Aufgabenteile d) und e)}

Zwischen der ersten und zweiten Szene bearbeiten C und D die Aufgabenteile d) und e). Um den roten Faden nicht zu verlieren, sollen ihre Herangehensweisen kurz beschrieben werden.

\section{Zu Aufgabenteil d)}

Beide lesen die Aufgabe und lachen (vgl. Szene 1, Zl. 44 - 45). C will die Bearbeitung von c) durchstreichen, wird aber von $D$ daran gehindert. $D$ betrachtet Blatt a). Nun argumentiert er, dass die Funktion für jeden Zeitpunkt wiedergibt, wie weit Jan Ulrich gefahren ist und die Geschwindigkeit von diesen Strecken irgendwie abhängig ist. Das sieht $C$ sofort ein. Nun wird auch Blatt b) zu Rate gezogen. Nun macht $C$ den Vorschlag, auch die Strecke für 15:59 Uhr zu berechnen, die Wegdifferenz zu 16 Uhr zu berechnen und dann zu teilen. Sie kann allerdings nicht angeben, wodurch sie teilen will. Sie meint schließlich, die Durchschnittsgeschwindigkeit einer Minute berechnet zu haben (19:30). David stimmt ihr zu: 
„D: Ja, klar geht auch, klar, weil wenn wir uns hier die Funktion so aufzeichnen würden. (zeichnet Treppenfunktion, vgl. Abbildung 11) Was wir hier haben wäre Durchschnittsgeschwindigkeit, fängt an erste halbe Stunde hier oben... (zeigt auf Treppenfunktion). Nur für diese Zeiträume und so ausgerechnet. Jetzt können wir diese so klein wie möglich

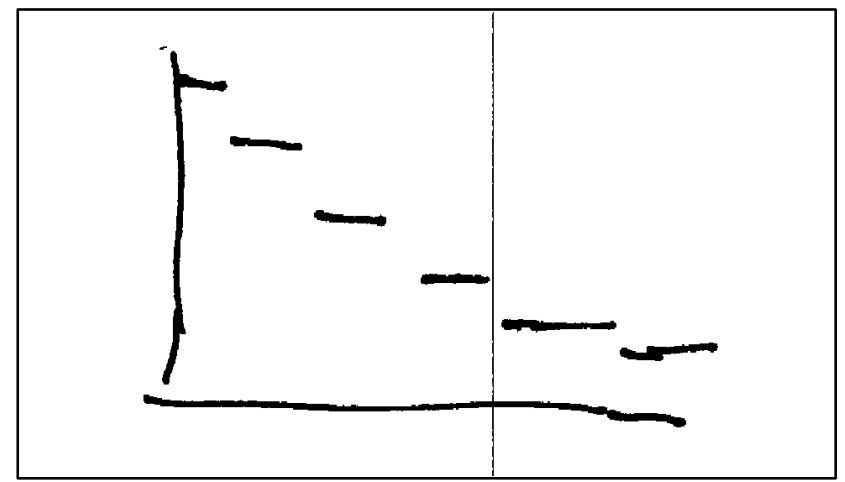
machen dann bekämen wir eine immer genauere Durchschnittsgeschwindigkeit. Dann hätten wir einen Näherungswert, der genau damit übereinstimmt. Das wäre die Lösung für die nächste Aufgabe.“133

Abbildung 11: D’s Treppenfunktion

Dem kann $\mathrm{C}$ folgen. Diese Idee wird nun als neue Bearbeitung für Aufgabe c) notiert, die alte Bearbeitung durchgestrichen. C schreibt die ersten 1 1/2Zeilen der neuen Bearbeitung.

D formuliert jetzt noch einmal, dass er eine Grenze des Zeitintervalls „gegen 16 Uhr streben lassen“ will, weil das "genau möglichste“ ist. Nun ergänzt D die neue Antwort auf c). Gemeinsam wird die Formulierung erarbeitet.

C macht nun den Vorschlag, sich von beiden Seiten zu nähern und den beidseitigen Grenzwert zu berechnen. D ist noch nicht zufrieden und betrachtet Blatt a). Nun erläutert C ihm noch einmal ihre Idee, die zurückgelegten Wege bzgl. 15:59 Uhr und 16 Uhr zu berechnen und die Differenz zu bestimmen. Dabei beschränkt sie sich nun auf das Nähern von einer Seite. D erinnert sich an Aufgaben aus dem Unterricht, wo der beidseitige Grenzwert berechnet wurde. Der Abstand aus dem Unterricht, der verringert wurde, wird nun mit der Zeit dieser Aufgabe identifiziert. C notiert nun die erste Zeile der Lösung auf Blatt d) und gerät ins Stocken. D beschreibt daraufhin noch einmal präzise das Näherungsverfahren, wobei er die Differenzenbildung im Zähler des Differenzenquotienten explizit erwähnt. C will eine Funktion aufstellen, die die Geschwindigkeit angibt, da man in die Differenz 16 Uhr nicht einsetzen kann. $D$ liest die Aufgabe noch einmal laut vor und $C$ stellt fest, dass sie gar nichts berechnen sollen. Also wird nun die Strategie der Verkleinerung des Zeitintervalls von C notiert. 


\section{Zu Aufgabenteil e)}

D liest die Aufgabe vor. C schlägt vor, „das jetzt mit dem Limes zu machen“134. D weist darauf hin, dass die gegebene Funktion $f$ eine bedeutende Rolle spielt und $\mathrm{C}$ notiert sie noch einmal auf Blatt e). Dann wird zunächst die Wegdifferenz mit der Durchschnittsgeschwindigkeit gleichgesetzt. Verbal formuliert $D$ nun die folgende Sichtweise des

Differenzenquotienten: $\lim _{h \rightarrow 0} \frac{f(x+h)-f(x)}{h}$. Nun beginnt D erneut, die Wegdifferenz zu notieren. C erkennt, dass die Differenz von zwei Wegen nicht die Durchschnittsgeschwindigkeit sein kann. D ergänzt ihre Lösung um den Nenner, den ihm C diktiert. Dann will er weiter eine allgemeine Lösung mit dem Limes notieren.

C will lieber ein Beispiel rechnen und beginnt, das Beispiel aufzuschreiben, indem sie die beiden Zeitgrenzen notiert. Rechts daneben wird nun der Abstand h berechnet und in einer neuen Zeile der Differenzenquotient aufgeschrieben. Dann wird die Funktion eingesetzt, to durch $\mathrm{t}$ - $\mathrm{h}$ ersetzt, ausmultipliziert und $\mathrm{h}$ wird gekürzt. Ein $\mathrm{h}$ verbleibt im Zähler. $\mathrm{C}$ weiß sofort, dass das nicht stört. $D$ will nun für $h$ wieder $t_{1}-t_{2}$ einsetzten. $C$ hält ihn davon ab. Nun wird $\mathrm{h}$ gleich 0 gesetzt, und für $\mathrm{t}_{1}$ wird 2 eingesetzt. Das Ergebnis ist negativ. Der VL lobt die beiden für den Rechenweg und vermutet, dass irgendwo ein Rechenfehler aufgetreten ist. Diesen Vorzeichenfehler wollen die beiden unbedingt finden, was ihnen auch gelingt.

Anhand des Terms nach der Grenzwertbildung erkennt C, dass die Werte für große t wieder negativ werden. $D$ weist darauf hin, dass die gegebene Funktion nur für $\mathrm{t}$ kleiner als 5 Stunden definiert ist. $\mathrm{C}$ setzt 5 ein und erhält 0 .

\section{Szene 2; Zeilen 140 - 172}

Nach der Partnerarbeitsphase hat der Versuchsleiter in den meisten Fällen noch ein Gespräch mit den Schülerinnen und Schülern geführt. Dort nahm er gelegentlich Argumente der Schülerinnen und Schüler aus der Partnerarbeitsphase auf und hakte nach, so auch in der folgenden Szene. Zwischen dem Ende der ersten Szene und diesem Abschnitt des Gesprächs liegen 33 Minuten. Insgesamt beschäftigen sich C und D jetzt schon über 50 Minuten mit der Aufgabe.

${ }^{134}[14,110]$ 
140 Gespräch zu Aufgabenteil c): Der VL lobt, dass sich D zunächst überlegt hat, dass es 141 eine Momentangeschwindigkeit gibt und erst dann eine Berechnungsmöglichkeit 142 sucht. Der VL liest die Bearbeitung vor (vgl. Abbildung 10) und fragt im Hinblick auf 143 diese, warum Jan Ulrich denn eine Momentangeschwindigkeit haben muss.

150 VL: Ja, wieso muss er das denn haben?

151 D: Aber.. Ja, eigentlich, Momentangeschwindigkeit gibt's ja in dem Sinne ja nicht, 152 weil, eine Geschwindigkeit gibt es ja immer erst dann, wenn man einen Weg 153 zurücklegt.

154 C: Eine Zeit schon längst wieder vorbei.

155 VL: Ja, zu einem Zeitpunkt, (D: Ja.) ne, zu einem Zeitpunkt vergeht keine Zeit. Kann 156 ich da überhaupt eine Geschwindigkeit haben?

157 D: Ich glaub nicht, weil äh, eine Geschwindigkeit ergibt sich erst daraus, dass man 158 eine Strecke zurücklegt.

159 VL: Ja, aber was hast du denn dann da gerade ausgerechnet?

160 C: Ja, aber in dem Moment ist er dann ja, in dem Moment wo er,.. zu dem Zeitpunkt 161 hat er je ne gewisse Geschwindigkeit drauf. ... Weg hat er vielleicht zurückgelegt, 162 aber die zeigt vielleicht ein Tacho (D: Ja.) würd das anzeigen. (VL: Aha.) Und der, 163 in dem Moment. (VL: Ehm. D: Ja, hatten wir..) Das hatten wir ausgerechnet....

164 D: (nuschelt) ...

165 VL: Ja ist es nun die Momentangeschwindigkeit oder nicht?

166 C: Doch!

167 D: (nuschelt) Ist es die,.. ja

168 C: Ist es. ... Weil ich ja in dem Moment, die genau diese Geschwindigkeit (klopft mit 169 einem Stift auf den Tisch und lässt die Stiftspitze auf einem Punkt), zu diesem 170 Zeitpunkt, ich habe in diesem Moment vielleicht keine Strecke zurückgelegt, 171 (bewegt den Stift über den Tisch) aber wenn ich das im Ganzen betrachte, würd 172 ich, bin ich in dem Moment so schnell.

Diese Szene soll unter den folgenden Gesichtspunkten betrachtet werden: Zunächst soll D's Wechsel der Sichtweise und die damit verbundene Meinungsänderung bzgl. der Existenz der Momentangeschwindigkeit betrachtet werden. Damit zusammen hängen C's Redebeiträge, die eine stark ausgeprägte einbettende Sichtweise erkennen lassen. In 
einem zweiten Abschnitt soll die Rolle des Versuchsleiters in dieser Szene betrachtet werden.

$D$ ändert seine Meinung; isolierende Sichtweise

\section{D's Wechsel der Sichtweise und C's einbettende Sicht}

Der VL liest inzwischen die Bearbeitung zu Aufgabenteil c) vor (vgl. Abbildung 10): „Da der Fahrer um 16:00 Uhr eine bestimmte Geschwindigkeit haben musste, gibt es auch eine Momentangeschwindigkeit." und fragt (Zl. 150): „Ja, wieso muss er das denn haben?“ Vergleicht man D’s Antwort mit der Meinung, die er in Szene 1 vertreten hat, ist man erstaunt (ZI. 151): „Aber.. Ja, eigentlich, Momentangeschwindigkeit gibt's ja in dem Sinne ja nicht..." In Szene 1 hat er die gegenteilige Meinung vertreten. Er begründet seine neue Einstellung so (ZI. 152): „...weil, eine Geschwindigkeit gibt es ja immer erst dann, wenn man einen Weg zurücklegt.“

Hier erkennt man den zweiten Teil der für die isolierende Sichtweise typische Argumentation in der r-Welt. Dabei wird „keine Momentangeschwin digkeit haben“ mit „Momentangeschwindigkeit =0“ identifiziert: $\mathrm{Zu}$ einem Zeitpunkt legt man keinen Weg zurück. Wenn man keinen Weg zurücklegt, hat man keine Momentangeschwindigkeit. (m-Welt-Version: Da die lokale Änderung gleich Null ist, gilt dies auch für die lokale Änderungsrate.) Vielleicht liefert $C$ den ersten Teil der Begründung, als sie direkt auf D's Argument antwortet (vgl. Zl. 154): „Eine Zeit, schon längst wieder vorbei.“

Gründe für D's Sichtweisenwechsel
Es stellt sich die Frage, ob man an dem Video erkennen kann, ob es eine Stelle gibt, an dem D seinen Sichtweisenwechsel vornimmt. Der Wechsel lässt sich aber nicht explizit ausmachen. Allerdings gibt es zwei Stellen zwischen den vorgestellten Szenen, die einen Anlass zum Sichtweisenwechsel bieten: Bei der Bearbeitung von Aufgabenteil d) zeichnet D eine Treppenstufenfunktion, die verschiedene Durchschnittsgeschwindigkeiten in gewissen Zeiträumen angibt, vgl. Abbildung 11. Seine Zeichnung kommentiert er so (vgl. D's Zitat im Abschnitt zu Aufgabenteil d)): „Jetzt können wir diese so klein wie möglich machen dann bekämen wir eine immer genauere Durchschnittsgeschwindigkeit. Dann hätten wir einen Näherungswert, der genau damit übereinstimmt.“ Hier formuliert D einen Grenzpro- 
zess, der damit endet, dass ein Näherungswert mit der Momentangeschwindigkeit genau übereinstimmt. Etwas später sagt er, dass das „das genau möglichste" sei. Es scheint inm klar zu sein, dass er nicht einfach den Zeitpunkt in seine Formel für die Durchschnittsgeschwindigkeit einsetzen kann, was zu der Idee führen kann, dass es die Momentangeschwindigkeit nicht gibt, da man sie nicht berechnen kann. Die zweite Ster le schließt inhaltlich und auch im zeitlichen Ablauf direkt daran an: $\mathrm{C}$ möchte eine Geschwindigkeit-Zeit-Funktion aufstellen, da man „16 Uhr nicht in die Differenz einsetzen kann." Sie macht damit D's Problem explizit.

Bei der Bearbeitung von Aufgabenteil c) behauptet D, dass man die Momentangeschwindigkeit nicht berechnen kann. Aufgrund der beiden aufgeführten Stellen des Aufgabenteils d) kann man vermuten, dass die Bemühungen, eine Berechnungsmöglichkeit zu finden, zu dem Sichtweisenwechsel geführt haben.

Nun greift der Versuchsleiter C's Bemerkung über die Zeit auf und fragt, ob es zu einem Zeitpunkt eine Momentangeschwindigkeit geben kann, vgl. Zl. 155 - 156. Daraufhin wiederholt D seine Argumentation (ZI. 157 - 158): „Ich glaub nicht, weil äh, eine Geschwindigkeit ergibt sich erst daraus, dass man eine Strecke zurücklegt." Mit Bezug auf die Bearbeitung von Aufgabenteil e) fragt dann der Versuchsleiter, was $C$ und D denn ausgerechnet haben.

Für C gibt es die Momentangeschwindigkeit;
Jetzt schaltet sich C ins Gespräch ein, indem sie zunächst an D's Argumente aus der ersten Szene erinnert (ZI. 160 - 161): „Ja, aber in dem Moment ist er dann ja, in dem Moment wo er,.. zu dem Zeitpunkt hat er je ne gewisse Geschwindigkeit drauf. ..." Dann fährt sie fort (ZI. 161): „Weg hat er vielleicht zurückgelegt,“. Sie erkennt D's Problem, dass es zu einem Zeitpunkt keine Ortsänderung gibt. Ihre Vorstellung von dem sich bewegenden Radfahrer ist aber so mächtig, dass die lokale Ortsänderung keine Rolle für sie spielt: Ob Weg zurückgelegt wurde? - „Vielleicht“, vielleicht im Sinne von „Ich will mich nicht festlegen." $C$ braucht sich auch nicht fest- 
legen, denn sie kann die Frage des Versuchsleiters nach dem, was berechnet wurde, beantworten: Die Zahl, die ein Tacho anzeigen würde, wurde berechnet, vgl. Zl. 162 - 163.

$D$ ist verwirrt

Auch $D$ befindet sich nun im inneren Konflikt: Zu einem Zeitpunkt, zu dem die Ortsänderung Null ist, kann es sehr wohl eine positive lokale OrtsÄnderungs-Rate geben, vgl. Zl. 164 und 167.

C's Argumente; Prototyp der einbettenden Sichtweise
Wegen D's Nuscheln fragt der Versuchsleiter noch einmal nach (ZI. 165): „Ja ist es nun die Momentangeschwindigkeit oder nicht?" $C$ ist sich sicher (ZI. 166): „Doch!“, D nicht (ZI. 167): ,,nuschelt) Ist es die,.. ja“ C versucht in ihrer folgenden Argumentation auf das scheinbare Paradoxon einzugehen. Zunächst zeigt sie D an, dass sie sein Problem verstanden hat (Zl. 168 - 179): „Weil ich ja in dem Moment, die genau diese Geschwindigkeit (klopft mit einem Stift auf den Tisch und lässt die Stiftspitze auf einem Punkt), zu diesem Zeitpunkt, ich habe in diesem Moment vielleicht keine Strecke zurückgelegt,“. Nicht nur mit Worten, sondern auch mit der Stiftspitze bedeutet sie einen Punkt bzw. Zeitpunkt. Die Formulierung „zu diesem Zeitpunkt, ich habe in diesem Moment vielleicht keine Strecke zurückgelegt" zeigt noch einmal deutlich, dass sie D verstanden hat, dass sein Argument für sie aber keine Rolle spielt, denn, so fährt sie fort (ZI. 170 - 172): „(bewegt den Stift über den Tisch) aber wenn ich das im Ganzen betrachte, würd ich, bin ich in dem Moment so schnell." Mit neuen Worten wiederholt sie D's Argument aus der ersten Szene („Also, wenn du Fahrrad fährst, hast du ja immer ne Momentangeschwindigkeit.". Auch diesen Redebeitrag unterstreicht sie durch eine Geste: Wurde zur Verdeutlichung eines Zeitpunkts (oder Orts) die Stiftspitze auf einer Stelle des Tisches gelassen, fährt sie nun mit ihr über den Tisch, um eine Bewegung aufzuzeigen.

C's Formulierung (ZI. 171 - 172) „aber wenn ich das im Ganzen betrachte, [...], bin ich in dem Moment so schnell." kann als Prototyp der einbettenden Sichtweise angesehen werden. Erst vor dem Hintergrund einer Bewegung über einem Zeitraum (als Ganzes) kann man sinnvoll von einer Momentan- 
C und die Denkwelten

geschwindigkeit zu einem Zeitpunkt denken und reden. Ihre ausgeprägte einbettende Sichtweise scheint sie gegen das Grundparadoxon „immun“ zu machen.

Besonders wichtig erscheint mir hier, dass es $C$ gelingt, die beiden Denkwelten in Einklang zu bringen: In der r-Welt gibt es Momentangeschwindigkeit, weil etwas, das sich bewegt, Momentangeschwindigkeit hat. Die Momentangeschwindigkeit würde sogar von einem Tacho angezeigt. In der m-Welt kann sie mit Hilfe der Diffe renzialrechnung die lokale Änderungsrate der Orts-Zeit-Funktion berechnen. Dieser theoretisch ermittelte Wert hat aber eine Bedeutung in der Realität. Er muss mit der Tachoanzeige übereinstimmen. D hat einen Sichtweisenwechsel zur isolierten Sichtweise hinter sich. Er hat nun Probleme, den berechneten Wert zu deuten: In den Zeilen 164 und 167 nuschelt er nur noch, was als Unsicherheit gedeutet werden kann, zumal er gerade in eine Falle des Versuchsleiters getappt ist, s.u. Man kann hier erkennen, dass es einen Zusammenhang zwischen der Wahl der Sichtweise und dem Vermögen, der m-Welt Sinnhaftigkeit zuzuschreiben, gibt. Die einbettende Sichtweise ermöglicht es, $r$ - und $\mathrm{m}$-Welt in Einklang zu bringen, die isolierende verbietet es, oder erschwert es zumindest deutlich.

\section{Die Rolle des Versuchsleiters in dem Gespräch}

Obwohl die Versuchsanordnung dieser Untersuchung eine klinische ist, nimmt der Versuchsleiter einen großen Einfluss auf die Schülerinnen und Schüler. Sie wissen, dass der Versuchsleiter „von der Mathematik an der Universität" kommt und dass er eine mathematische Untersuchung vor hat.

Wahrscheinlich beeinflusst dieses Wissen das Denken und Handeln der Schülerinnen und Schüler, da sie vielleicht versuchen zu berücksichtigen, welche Erwartungen der Versuchsleiter an sie hat.

Der VL beeinflusst das Gespräch
In dieser Szene nimmt der Versuchsleiter aber nachweislich deutlicher Einfluss. Zunächst greift er die schriftliche Antwort zu Aufgabenteil c) auf und 
fordert eine weitere Begründung, vgl. ZI. 150. D's Reaktion ist die Betonung der isolierenden Sichtweise. C denkt an Zeitpunkte. Den Zeitpunkt greift der Versuchsleiter wieder auf und fragt (ZI. 157 - 158): „Kann ich da überhaupt eine Geschwindigkeit haben?“ Selbst wenn er das Wort „überhaupt" weggelassen hätte, kann sich D an dieser Stelle in seiner Meinung nur bestärkt sehen. Die Formulierung mit dem „überhaupt“ fordert die Antwort „Nein“ heraus. Der Versuchsleiter suggeriert, dass es keine Momentangeschwindigkeit gibt.

Wie sich im Weiteren zeigt, kann diese Frage auch als das Aufstellen einer Falle interpretiert werden, denn diese lässt der Versuchsleiter zuschnappen, wenn er weiter fragt (ZI. 159): „Ja, aber was hast du denn dann da gerade ausgerechnet?" Der Hinweis auf den berechneten Wert legt nahe, dass der Versuchsleiter meint, es gäbe doch eine Momentangeschwindigkeit.

Die vierte Nachfrage durch den Versuchsleiter kann in diesem Sinne als neutral angesehen werden. $C$ hat den berechneten Wert mit der Tachoanzeige in Verbindung gebracht, D befindet sich in dem inneren Konflikt. Der Versuchsleiter wiederholt inhaltlich die letzte Frage und richtet sie wohl an D. 


\section{Ein Blick in Schulbücher}

In diesem Abschnitt werden zunächst bei einigen Schulbüchern die Kapitel zur Einführung in die Differenzialrechnung im Hinblick auf die isolierende und einbettende Sichtweise betrachtet. Anschließend werden aus den in dieser Arbeit gewonnenen Erkenntnissen Konsequenzen für die Schulpraxis gezogen.

Folgende Schulbücher wurden gesichtet (chronologische Reihenfolge der Veröffentlichung): [Athen/Griesel 1979], [Glaser/Scheid/Wellstein 1983], [Kroll 1985], [Schmid/ Schweizer 1990], [Hahn/Dzewas 1994], [Baumann 1998], [Griesel/Postel 1999].

\subsection{Einstiege in die Differenzialrechnung}

Einleitend kann festgehalten werden, dass das Sekanten-Tangentensteigungsproblem in jedem Werk auftaucht. In allen Werken, bis auf [Schmid/Schweizer 1990] bildet die Frage nach der Tangentensteigung den Einstieg in die Differenzialrechnung. Schmid und Schweizer stellen die Frage nach der Momentangeschwindigkeit und visualisieren die Situation anschließend sofort mittels des Graphs der Weg-Zeit-Funktion und mit Tangenten. In einigen anderen Werken lassen sich Bewegungsaufgaben als Anwendungen finden. Drei Konzepte lassen sich in den berücksichtigten Schulbüchern aus machen:

Konzept 1: Tangentensteigung ([Kroll 1985], [Hahn/Dzewas 1994], [Griesel/Postel 1999]):

Der aus der Mittelstufe bekannte Tangentenbegriff wird wiederholt und auf Kreise und Parabeln angewendet, vgl. Abbildung 12. Die Frage nach der Steigung oder der Richtung von beliebigen Kurven öffnet das eigentliche Problemfeld. Dass eine Kurve in einem Punkt überhaupt eine Richtung hat, wird nicht in Frage gestellt, sondern vorausgesetzt: 
„Bekanntlich spricht man auch bei krummlinigen Kurven von einer Richtung, etwa wenn man sagt, daß man ,eine bestimmte Richtung gerade eingeschlagen hat' oder daß man ,in einer bestimmten Richtung im Augenblick fährt oder fliegt'." [...] Versucht man, „in diesen Punkten die jeweilige Kurvenrichtung so genau wie möglich zu bestimmen, so wird jeder vermutlich mit Hilfe eines Pfeils bzw. eines kleinen Geradenstücks, das im betreffenden Punkt ansetzt, dem Kurvenverlauf möglichst gut zu folgen suchen. “135

„Die Steigung eines Graphen verändert sich von Punkt zu Punkt.، “136

Dieses Problem haben wir in diesem Buch schon für einige Spezialfälle einer Funktion $\mathrm{f}$ behandelt.

(1) $f(x)=a \cdot x^{2}$.

Der Graph dieser Funktion ist eine Parabel (siehe Seite 49). Die Steigung der Tangente an der Stelle $\mathrm{x}_{0}$ beträgt:

$\mathrm{m}=2 \mathrm{ax}_{0}$

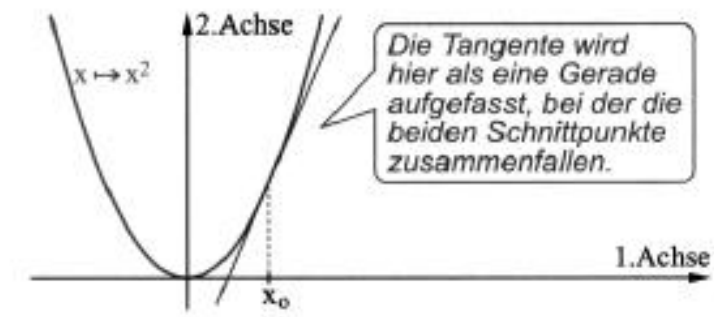

(2) $f(x)=\sqrt{r^{2}-x^{2}}$.

Der Graph dieser Funktion ist ein Halbkreis (siehe Seite 62). Die Steigung der Tangente an der Stelle $\mathrm{x}_{0}$ beträgt:

$\mathrm{m}=-\frac{\mathrm{x}_{0}}{\mathrm{y}_{0}}=-\frac{\mathrm{x}_{0}}{\sqrt{\mathrm{r}^{2}-\mathrm{x}_{0}^{2}}}$

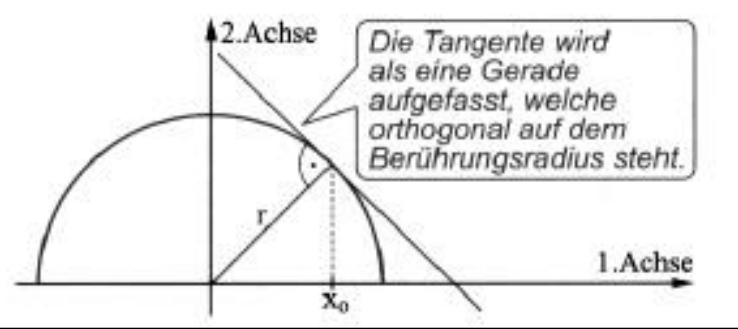

Abbildung 12: Tangenten an Parabeln und Kreisen, [Griesel/Postel 1999], S. 146

Es wird also versucht, Intuitionen bezüglich der Eigenschaft „Steigung einer Kurve“ zu unterstützen. Weiterhin werden diese Intuitionen genutzt, um die Existenz der Tangentensteigung zu begründen. Es wird vorausgesetzt, dass Schülerinnen und Schüler Kurven in jedem Punkt intuitiv eine Steigung bzw. Richtung zugestehen und sich somit Tangenten in jedem Punkt vorstellen können.

Anschließend wird der Begriff der Tangente erweitert: Zunächst „darf“ eine Tangente nun auch den Graphen schneiden (Standard-Beispiel: Der Graph von $\mathrm{x} \mapsto \mathrm{x}^{3}$ an der Stelle $x=0$ ). Weiterhin wird die Eigenschaft „Tangente-sein“ als lokale Eigenschaft gesehen (Eine Gerade kann an einer Stelle Tangente sein und den selben Graphen an anderer Stelle schneiden.), vgl. Abbildung 13.

\footnotetext{
${ }^{135}$ [Kroll 1985], S. 31

136 [Griesel/Postel 1999], S. 147
} 
Der Wert der Ableitung einer Funktion an einer Stelle wird mit dem Wert der Tangentensteigung identifiziert, vgl. Abbildung 14, untere Definition. Dann stellt sich die Frage nach der Berechnung der Tangentensteigung. Dazu wird ein Grenzprozess der Sekantensteigungen betrachtet. Ein „intuitiver Grenzwertbegriff“ hilft, den Wert zu bestimmen. Zum formalen Grenzwertbegriff heißt es z.B.: „Im Anhang auf Seite 246 wird der Begriff der Ableitung mithilfe von Folgen präzisiert.“ ${ }^{137}$

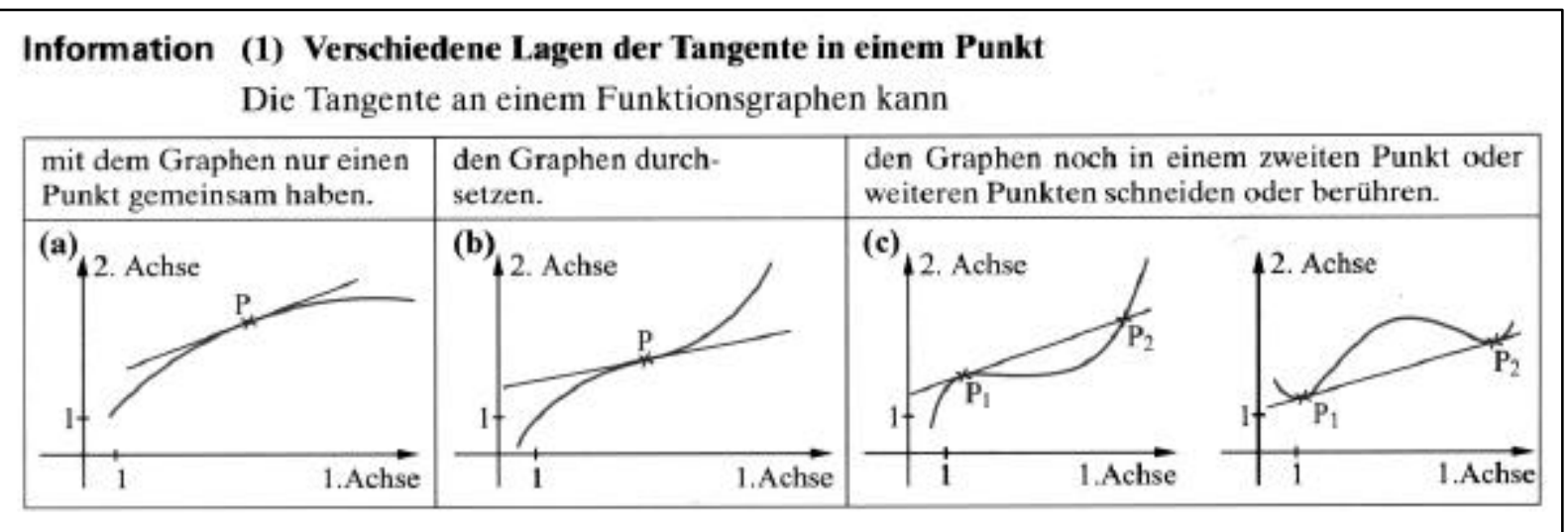

Abbildung 13: Erweiterung des Tangentenbegriffs, [Griesel/Postel 1999], S. 148

Damit kommen wir nun auch zu einer endgültigen Festlegung des Tangentenbegriffs:

Definition: Eine Gerade $t$ nennen wir Tangente an den Graphen von $f$ im Punkt $P_{0}\left(x_{0} ; f\left(x_{0}\right)\right)$, wenn sie folgende Eigenschaften hat:

(1) Die Gerade $t$ hat mit dem Graphen von $f$ den Punkt $P_{0}$ gemeinsam.

(2) Die Steigung von $t$ ist $f^{\prime}\left(x_{0}\right)$.

Wir übertragen den Steigungsbegriff von den Tangenten an Funktionsgraphen auf die Graphen selbst:

Definition: Unter der Steigung eines Graphen an einer Stelle $x_{0}$ versteht man die Steigung der Tangente an dieser Stelle.

Die Ableitung einer Funktion gibt also die Steigung der Tangente und damit des Graphen an der betrachteten Stelle an. Sie ist der Grenzwert der Sekantensteigungen.

Auch das Problem der Tangentengleichung ist nun gelöst.

Abbildung 14: Der Zusammenhang von Ableitung und Tangentensteigung, [Hahn/Dzewas 1994], S. 136 
Zu Verwirrung kann es bei Schülerinnen und Schülern kommen, wenn (wie z.B. bei Hahn und Dzewas) zunächst die Tangente, bzw. ihre Steigung mit Hilfe des Werts der Ableitung definiert wird, wobei die Ableitung (intuitiv) als Steigung des Graphs in einem Punkt eingeführt wurde, dann aber die Steigung des Graphs wiederum mit Hilfe der Tangentensteigung erklärt wird, vgl. Abbildung 14. Der Unterschied zwischen einer Definition und der Beschreibung einer Intuition muss klar heraus gearbeitet werden.

Ähnlich, wie die rWelt-Vorstellungen die Existenz der Momentangeschwindigkeit sichern, werden hier Eigenschaften einer intuitiv immer vorhandenen Tangente bemüht. Dass aber Schülerinnen und Schüler aufgrund einer isolierenden Sichtweise Probleme mit dem Tangentenbegriff haben können, bleibt unberücksichtigt. Es wird davon ausgegangen, dass die Schülerinnen und Schüler wie für die Momentangeschwindigkeit auch einen Wert für die Tangentensteigung erwarten und berechnen.

Konzept 2: Lückenwert ([Athen/Griesel 1978], [Glaser/Scheid/Wellstein 1983]):

In beiden Lehrwerken findet man ausführliche Kapitel zu den Themen Grenzwerte von Folgen und Funktionen. Athen und Griesel führen der Wert der Tangentensteigung als Lückenwert der Sekantensteigungsfunktion, also der Differenzenquotientenfunktionen ein, vgl. Abbildung 16. Der Grenzwert wird als Lückenwert an der besagten Stelle gesehen. Glaser, Scheid und Wellstein definieren den Grenzwert von stetigen Funktionen als Lückenwert schon im Kapitel „Stetigkeit“, vgl. Abbildung 15.

\section{Der Grenzwert einer Funktion an der Stelle $x_{0}$ ist diejenige Zahl, die von den Werten an den benachbarten Stellen an- genähert wird. Der Punkt $\left(x_{0} \mid c\right)$ ist derje- nige Punkt, der in den bei $x_{0}$ unterbro- chenen Graph paBt (Abb. 5).}

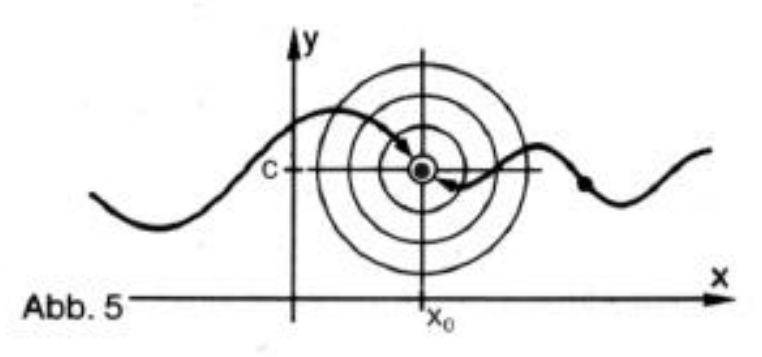

Abbildung 15: Der Grenzwert stetiger Funktionen als Lückenwert, [Glaser/Scheid/Wellstein 1983], S. 30

Die Formulierung „benachbarte Stellen“ (vgl. Abbildung 15) kann jedoch höchstens auf einer intuitive Ebene genutzt werden: Schülerinnen und Schüler kennen benachbarte Zahlen aus der Grundschule bezüglich der natürlichen Zahlen. 4 und 6 sind die 
Nachbarn der Zahl 5. andererseits haben sie gelernt, dass zwischen zwei verschiedenen rationalen Zahlen immer eine weitere rationale Zahl liegt, man also in dieser Zahlenmenge und allen, die diese enthalten, nicht mehr von Nachbarschaft reden kann.

9.2. Berechnung der Tangentensteigung bei verschiedenen Funktionen

Aufgabe 1: Funktion $x \mapsto x^{3}$; Stelle a

a) Bestimme die Steigung der Tangente an den Graphen der Funktion $x \mapsto x^{3}$ an der Stelle a.

b) Bestätige das Ergebnis durch Ausfüllen der Lücke im Graphen der Sekantensteigungsfunktion.

Lösung: a) (1) Steigung $s k(x)$ der Sekante durch $P=p\left(a ; a^{3}\right)$ und $S=p\left(x ; x^{3}\right)$

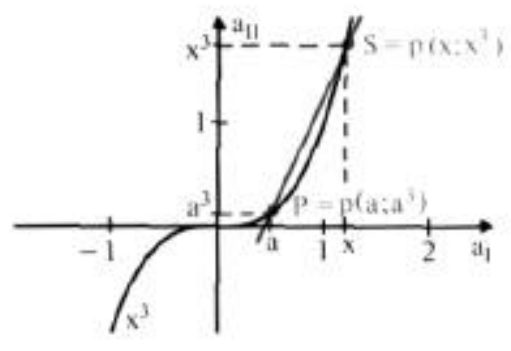

$s k(x)=\frac{x^{3}-a^{3}}{x-a}=x^{2}+x a+a^{2} \quad($ für $x \neq a)$

(2) Steigung der Tangente im Punkt $P$

Wandert $\mathrm{S}$ auf $\mathrm{P} z \mathrm{zu}$, so unterscheidet sich $\mathrm{x}$ immer weniger von a und $s \mathrm{k}(\mathrm{x})$ immer weniger von

$a^{2}+a \cdot a+a^{2}=3 a^{2}$.

Es gilt: $\lim s k(x)=3 a^{2}$.

$3 \mathrm{a}^{2}$ ist $\stackrel{x \rightarrow a}{\operatorname{ser}}$ Grenzwert von $\mathrm{sk}(\mathrm{x})$ für $\mathrm{x}$ gegen $\mathrm{a}$.
Die Steigung der Tangente im Punkt $P$ beträgt $3 \mathrm{a}^{2}$

b) (3) Bestätigung des Ergebnisses durch Ausfüllen der Lïcke im Graphen der Sekantensteigungsfunktion $[x \mapsto s k(x) ; x \neq a]$

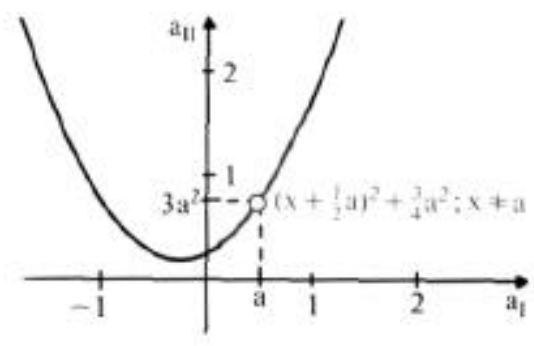

Beachte für die Zeichnung des Graphen:

$\operatorname{sk}(x)=x^{2}+a x+a^{2}=\left(x+\frac{1}{2} a\right)^{2}+\frac{3}{4} a^{2}$.

Der Scheitelpunkt der Parabel ist: $p\left(-\frac{1}{2} a ; \frac{3}{4} a^{2}\right)$.

$3 \mathrm{a}^{2}$ füllt die Lücke aus.

Abbildung 16: Der Wert der

Tangentensteigung als Lückenwert, [Athen/Griesel 1978], S. 118

Die Idee des Lückenwerts habe ich ebenfalls in Kapitel 4.2 entwickelt. Im Vergleich zu den rein innermathematischen Darstellungen in den Schulbüchern spielt bei mir jedoch die r-Welt für die Ausbildung notwendiger Intuitionen eine wichtige Rolle.

Konzept 3: Momentangeschwindigkeit ([Schmid/Schweizer 1990]):

Auch in diesem Schulbuch gehen der Differenzialrechnung Kapitel über Grenzwerte bei Folgen und Reihen voran. Als Einstiegsproblem in die Differenzialrechnung wird nach der Momentangeschwindigkeit einer Kugel, die eine schiefe Ebene hinunter läuft, gefragt. Die Frage, ob es für die Schülerinnen und Schüler so etwas wie eine Momentangeschwindigkeit gibt, wird nicht diskutiert. Offensichtlich wird diese Einsicht in die Existenz der Momentangeschwindigkeit bei den Schülerinnen und Schülern als 
gegeben vorausgesetzt. Der Wert der gesuchten Momentangeschwindigkeit wurde jedoch zuvor mit dem Tachostand eines Autos in Verbindung gebracht. Dann wird der Grenzwert der Durchschnittsgeschwindigkeiten-Funktion berechnet.

zwei Zeitpunkten. In Fig. 83.2 wurde deshalb versucht, die zum Zeitpunkt $t_{0}=1,5$ vorhandene "Schnelligkeit" der Kugel über ihre mittleren Geschwindigkeiten in den Zeitintervallen

$[1,5 ; 2,5] ;[1,5 ; 2] ;[1,5 ; 1,6] ;[1,5 ; 1,51]$; $[1,5 ; 1,501] ; \ldots$ anzunähern.

Die zugehörigen mittleren Geschwindig-

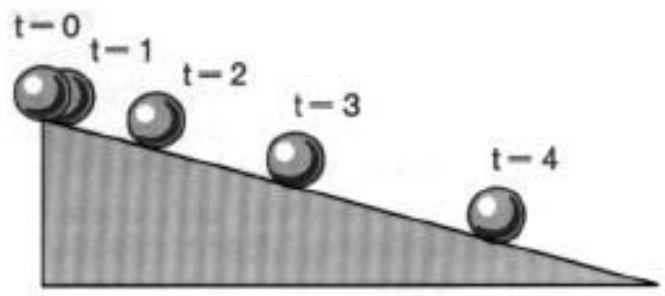
keiten (in $\frac{\mathrm{m}}{\mathrm{s}}$ )

0,$8 ; 0,7 ; 0,62 ; 0,602 ; 0,6002 ; \ldots$ scheinen dem Grenzwert 0,6 zuzustreben. Dies ist der gesuchte .,Tachostand" zum Zeitpunkt $\mathrm{t}_{0}=1,5$; er ergibt sich also als Grenzwert von mittleren Geschwindigkeiten.

Das Vorgehen läßt sich so zusammenfassen: Zuerst berechnen wir für die Zeitspanne zwischen $t_{0}-1,5$ und einem beliebigen Zeitpunkt $t \neq 1,5$ die mittlere

Geschwindigkeit

$$
v_{m}=\frac{s(t)-s\left(t_{0}\right)}{t-t_{0}}=\frac{0,2 \cdot t^{2}-0,2 \cdot 1,5^{2}}{t-1,5}=0,2 \cdot(t+1,5) .
$$

Danach untersuchen wir das Verhalten von $v_{m}$ für $t \rightarrow t_{0}$, also $t \rightarrow 1,5$.

Es zeigt sich: $\lim _{\mathrm{t} \rightarrow 1,5} \mathrm{v}_{\mathrm{m}}=\lim _{\mathrm{t} \rightarrow 1,5}(0,2 \cdot(\mathrm{t}+1,5))=0,6$.

Bei einer Bewegung nach dem Weg-Zeit-Gesetz $t \mapsto s(t)$ heiBt der Grenzwert

$$
\lim _{i \rightarrow t_{0}} \frac{s(t)-s\left(t_{0}\right)}{t-t_{0}}
$$

Momentangeschwindigkeit $v\left(t_{0}\right)$ zum Zeitpunkt $t_{0}$.

Abbildung 17: Der Wert der Ableitung als Momentangeschwindigkeit, [Schmid/Schweizer 1990], S. 83

Das Buch von Baumann ist kein Unterrichtswerk im Sinne eines Lehrbuchs. Der Untertitel heißt „Ein Arbeitsbuch mit Derive“. Der Autor stellt einen Vorschlag vor, wie das CAS Derive in den Analysisunterricht durchgängig eingebunden werden kann. Auf dieses Buch wird in Kapitel 6.2 eingegangen.

Beachtet man die Erscheinungsdaten der Schulbücher, kann man folgendes erkennen: Je weiter die „Strengewelle“ der 70er Jahre zurückliegt, desto weniger wird auf 
einen formalen Grenzwertbegriff Wert gelegt. Als Beispiel für diese Entwicklung können die Schulbücher [Griesel/Postel 1988] und [Griesel/Postel 1999] gesehen werden: Die Unterrichtseinheiten über Folgen, Reihen und ihre Grenzwerte sowie Stetigkeit stellen in dem Werk von 1988 eigenständige Kapitel dar, die sich im Buch an den Stellen einordnen, die der kanonische Aufbau der Analysis nahe legt. In dem Schulbuch von 1999 wird das Kapitel über Folgen, Reihen und Grenzwerte in den Anhang verdrängt, Stetigkeit wird gar nicht mehr behandelt.

\subsection{Sichtweisen und Denkwelten in den Schulbüchern}

Die Rolle der r-Welt übernimmt in den hier betrachteten Büchern zumeist die g-Welt der Kurven und Funktionsgraphen. Intuitionen über die Eigenschaft „Steigung“ in dieser Welt werden durchaus angesprochen. Ohne dass ein der einbettenden Sichtweise entsprechender Begriff verwendet wird, soll durch die Intuitionen die Existenz der lokalen Steigung einer Kurve gesichert werden.

Klar ist, dass man in jedem Schulbuch Formulierungen und Visualisierungen finden kann, die als einbettende Sichtweise interpretiert werden können. Das ist im Wesen der betrachteten Mathematik begründet. Beispiele dafür sind folgende, schulbuchpräzise Formulierungen: „Kurvenrichtung“ (Es heißt richtigerweise nicht „Punktrichtung“), „Steigung des Funktionsgraphs“, „Steigung des Graphs in einem Punkt“, „Steigung der Tangenten am Graphen“ ${ }^{138}$. Stets wird die Kurve als Referenz betont.

Auf die isolierende Sichtweise und die damit verbundenen Probleme wird in nur einem der betrachteten Werke eingegangen. Baumann problematisiert sie mit Hilfe der „Steigung eines Punktes“. Die fiktive Schülerin Mathilde erklärt dem ebenso fiktiven Schüler Nikolaus, was unter der „Grenzlage der Sekante“ zu verstehen ist:

„Nikolaus und Mathilde führen anlässlich der Tangentendefinition folgendes Gespräch:

- Nikolaus: Heute haben wir „Tangente“ als „Grenzlage von Sekanten“ definiert, aber ich raff' das irgendwie nicht.

${ }^{138}$ [Athen/Griesel 1979], Kap. 9.1; [Kroll 1985], S. 32; [Kroll 1985], S. 33; [Griesel/Postel 1999], S. 147 
- Mathilde: Was verstehst du denn nicht?

- Nikolaus: Ja ... - solange $Q$ mit $P$ nicht zusammenfällt, ist die Sekante keine Tangente. Sobald aber $Q$ wrklich „in $P$ hineingerückt“ ist, wie Lehrer Höfler sich aus zudrücken liebt, gibt es keine zwei Punkte mehr - dann gibt es aber auch keine Richtung mehr!

- Mathilde: Willst Du damit sagen, dass die Richtung der Tangente unbestimmt ist?

- Nikolaus (zögernd): Ja ... wenn nur noch ein Punkt P da ist, kann man durch inn Geraden in beliebiger Richtung zeichnen ... -

- Mathilde: - ... die alle Anspruch darauf erheben könnten, aus jenen Sekanten hervorgegangen zu sein. Das wolltest du doch sagen?

- Nikolaus: Ja, genau ... - die sich also alle mit gleichem Recht „Tangente“ nennen dürften.

- Mathilde: Du hättest recht, wenn es nur darauf ankäme, dass aus $P$ und $Q$ der eine Punkt $P$ entsteht.

- Nikolaus: Freut mich, dass ich recht hätte ...

- Mathilde: Aber darauf kommt's eben nicht an!

- Nikolaus: Schade! Worauf kommt's denn an?

- Mathilde: Es kommt darauf an, wie der Nachbarpunkt $Q$ an $P$ heranrückt!

- Nikolaus: Aha ... -?-

- Mathilde: $\mathrm{Q}$ rückt doch wohl nicht beliebig an $\mathrm{P}$ heran - oder?

- Nikolaus: Nee - beliebig wohl nicht ... -

Ü1: Auf welche Weise rückt $Q$ an $P$ heran? (Rekapitulieren Sie die Arbeitsaufträge 2 und 3.)

- Mathilde: Richtig, durch stetige Führung längs des Kurvenbogens PQ.

- Nikolaus: „Stetige Führung“ ... - klingt gut - aber was heißt denn das?

- Mathilde: Pass' aus. Wie zeichnest du von Hand eine Tangente an einen Kreis im Punkt P?

- Nikolaus: Ich nehme das Lineal und lasse die Linealkante durch P gehen.

- Mathilde: Vermutlich wird sie zuerst den Kreis in einem weiteren Punkt schneiden?

- Nikolaus: Kann sein. Solange dies der Fall ist, drehe ich das Lineal, bis... -

- Mathilde: - ... bis beide Schnittpunkte mit dem Auge nicht mehr zu unterscheiden sind.

- Nikolaus: Dann mache ich Halt.

- Mathilde: Dieses Haltmachen in der Drehung aber ist etwas ganz anderes, als wenn Du gesagt hättest: ich will nur den Punkt P! 
- Nikolaus: Mit diesem „Haltmachen in der Drehung“ ist die Tangente also festgelegt?

- Mathilde: Richtig. Ihr habt es (in der Schule) als "Grenzlage“ bezeichnet,

- Nikolaus: So ist das also - na schön ...“139

Hier tritt die isolierende Sichtweise in dem Gewand "Steigung eines Punktes" auf. Die Einbettung soll durch die „stetige Führung“ des Lineals an der Kurve stattfinden. Es bleibt festzuhalten, dass die Phänomen-Ebene nicht verlassen wird, das heißt, dass beide Sichtweisen in einer Einkleidung aufgeführt, aber selber nicht thematisiert werden. Im folgenden Kapitel wird begründet, warum sie thematisiert werden sollten.

\subsection{Konsequenzen für die Schulpraxis}

In den in Kap. 5 vorgestellten Transkripten zeigen Katharina und David folgendes Phänomen: Bei der ersten Bearbeitung des Aufgabenteils c) nutzen sie spontan die einbettende Sichtweise. In den Interpretationen habe ich das den „naiven Geschwindigkeitsbegriff" genannt. Auf ähnlich naiven Vorstellungen von Steigung und Geschwindigkeit bauen offensichtlich auch die Schulbücher auf. Aufgrund der Aufgabenstellung dieser Untersuchung und durch das Gespräch mit dem Versuchsleiter findet ein intensives Nachdenken über den Geschwindigkeitsbegriff statt. Das führt bei Katharina und David in das Grundparadoxon, den inneren Konflikt zwischen zwei logischen Argumentationsmustern in den beiden Sichtweisen. Es ist offensichtlich die Aufgabe, die die Schülerinnen und Schüler in diese Verwirrung stürzt. In den Schulbüchern (abgesehen von [Baumann 1998]) wird versucht, die isolierende Sichtweise gar nicht erst aufkommen zu lassen.

\section{Die Sichtweisen - Ein Thema für den Unterricht?}

Es stellen sich folgende Fragen: Sollten die Sichtweisen als Phänomene in der Schulpraxis thematisiert werden? Sollten Schülerinnen und Schüler zeitweilig auch die isolierende Sichtweise kennen lernen, was unweigerlich mit einer zeitweiligen Ver-

${ }^{139}$ [Baumann 1998], S. $137-138$ 
wirrung durch den Konflikt zwischen den Sichtweisen einher ginge? Ist dieser ein notwendiges geistiges Hindernis oder eine Denkhürde ${ }^{140}$, die bei der Erarbeitung des Grenzwertbegriffs genommen werden muss? Sollen die Sichtweisen auf einer Metaebene selber im Unterricht auftauchen, und wenn ja, wann?

Meiner Meinung nach sollte auf eine Diskussion der Sichtweisen auf der Phänomenund der Metaebene im Unterricht nicht verzichtet werden. Gründe dafür sind:

\section{(1) Die isolierende Sichtweise tritt auf:}

Nicht nur in den ausführlich besprochenen Transkripten legen Schülerinnen und Schüler die isolierende Sichtweise an den Tag. Sie ist ein uraltes Phänomen: Man kann verschiedene Paradoxien auf die isolierende Sichtweise zurückführen, vgl. 4.5 und 5.1. Auch Newton warnt davor, Zähler- und Nenner des Differenzenquotienten getrennt zu betrachten, was für die isolierende Sichtweise typisch ist. Er muss gegen den Einwand argumentieren, dass es kein letztes Verhältnis gibt, da es keine letzten Größen gibt, vgl. 1.2. Beide Sichtweisen, die isolierende und die einbettende, scheinen dem menschlichen Denken nahe zu liegen. Selbst wenn man die isolierende Sichtweise im Unterricht vermeidet, muss man damit rechnen, dass aufmerksame Schülerinnen und Schüler sie z.B. im Rahmen von Hausaufgaben selbständig entwickeln. Auch die im Unterricht unvermeidlichen Wortfaulheiten können die isolierende Sicht betonen und den Konflikt auslösen: Aus „Steigung des Graphs in einem Punkt“ über „Steigung in einem Punkt“ wird z.B. schnell „Steigung des Punkts“.

Die isolierende Sichtweise kann also meines Erachtens nicht vermieden und sollte daher nicht ignoriert werden. Daher sollten Lehrpersonen in der Lage sein, sie zu erkennen, um angemessen reagieren zu können. Auch Schülerinnen und Schüler sollten die beiden Sichtweisen erkennen können, wenn der verständige Umgang mit Mathematik ein Ziel des Mathe matikunterrichts sein soll. Denn wenn die Sichtweisen nicht nur als Phänomene auftreten, sondern mit eigenen Namen als Begriffe expliziert werden, können die Schülerinnen und Schüler in der Lage sein, auf einer Meta ebene über die Phänomene nachzudenken. Dann wiederum können sie bewusst die einbettende Sichtweise einnehmen.

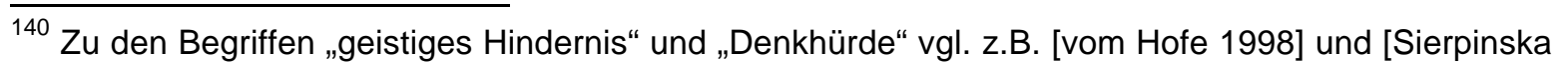
1992], aber auch [Hefendehl-Hebeker 1989]; der Begriff „geistiges Hindernis“ geht auf [Brousseau 1983] zurück.
} 
(2) Zusammenhänge zwischen r- und m-Welt

(2a) adäquate Vorstellungen:

Auch unabhängig von Mathematik ist die Idee, dass es positive lokale Änderungsraten gibt, auch wenn keine lokale Änderung stattfindet, Teil eines möglichen Weltbildes, das von heute akzeptierten physikalischen Vorstellungen (nicht-relativistisch, nichtquantenphysikalisch) getragen wird und zur Wahrnehmung alltäglicher Bewegungsvorgänge passt. Dafür ist die einbettende Sichtweise notwendig: Man muss einen Vorgang an einer Stelle beobachten, ohne ihn als Ganzes aus den Augen zu verlieren. Auch die Frage nach der jeweiligen Bedeutung der lokale Änderungsrate gehört zur Erschließung der Lebensumwelt: Welche Bedeutung hat der Lückenwert der Durchschnittswerte-Funktion (Diffe renzenquotientenfunktion) in dieser Situation? Wie genau ist der Lückenwert? - Durch die Analyse mit Hilfe der Sichtweisen erhoffe ich mir eine Vertiefung des Verständnisses des Zusammenhangs von r-Welt und dem zugehörigen Modell in der m-Welt, der Differenzialrechnung, bei den Schülerinnen und Schülern. Falls diese später überhaupt noch einmal in einen Konflikt geraten sollten, können sie sich aufgrund des Metawissens selber fragen, welcher Sichtweise welche ihrer Argumente zugehören, und welche Sichtweise der betrachteten außermathematischen Situation angemessen ist.

\section{(2b) Bedeutung von Vorstellungen:}

Von der Kenntnis der Sichtweisen erhoffe ich mir eine Verbesserung der Vorstellungen in beiden Denkwelten mit den beschriebenen Vorteilen für Schülerinnen und Schüler. Mit einer „verbesserten Vorstellung“ in der $\mathrm{m}$-Welt ist ins besondere nicht eine formale Grenzwertdefinition gemeint. Würde man die hier gestellte Aufgabe für einen Einstieg in die Differenzialrechnung verwenden, so würde man durch die dadurch angeregten Überlegungen auch keine formale Grenzwertdefinition gewinnen. Über die Bedeutung einer tragfähigen Vorstellung sagt vom Hofe:

\footnotetext{
„Nach heutigem Wissensstand sind - wie insbesondere Fischbein in zahlreichen Untersuchungen gezeigt hat - mathematische Problemlöseprozesse, auch auf höherem Niveau, stets mit intuitiven Vorstellungen und Begleitannahmen verbunden, die den Lösungsweg mehr oder weniger unbewußt beeinflussen. Insofern gibt es kein Denken ohne Vorstellungen. Im günstigen Falle können solche Vorstellungen mathematisches Denken positiv beeinflussen. Sie können jedoch auch [...] in die Irre
} 
führen, wenn sich Fehlvorstellungen zu unbewußt wirksamen „tacit models“ verfestigen. Es stellt sich daher die Frage, wie man mit diesem intuitiven Bereich umgeht, ob man etwa annimmt, daß sich adäquate Vorstellungen bei einem angemessenen formalen Umgang mit Mathematik von selbst einstellen, oder ob man die Ausbildung adäquater Vorstellungen bewußt begleitet und fördert.

Vieles spricht dafür, sich gründlicher als bisher mit der Entwicklung des intuitiven Bereichs zu befassen." ${ }^{141}$

Eine Vertiefung des Verständnisses des im Lehrplan geforderten „anschaulichen Grenzwertbegriffs" 142 kann meines Erachtens durch die Thematisierung der Sichtweisen erreicht werden.

\section{(2c) Das Bild von Mathematik:}

Die Differenzialrechnung ist ein schönes Beispiel dafür, wie aus außermathe matischen Situationen Mathematik entstehen kann, wie eine Alltagssituation Eigenschaften mathematischer Objekte und Eigenschaften nahe legt. Da Schülerinnen und Schüler die Mathematik oft als die Disziplin ewiger, festgeschriebener Wahrheiten sehen, als Formelgebäude ohne Bedeutung, müssen sie die r-Welt „verbiegen“. Z.B. bei der Rückinterpretation eines Rechenergebnisses einer Anwendungsaufgabe kann man gelegentlich beobachten, dass Schülerinnen und Schüler Vorstellungen von der r-Welt „verbiegen“, damit ein Rechenergebnis „passt“. Nur selten wird in Frage gestellt, ob die verwendete Mathematik problemangemessen war, oder ob ein Rechenfehler vorliegt. - Auch in dieser Untersuchung tritt dieses „Verbiegen“ auf: Fast alle Schülerinnen und Schüler sind zunächst der Meinung, dass es Momentangeschwindigkeit gibt („,naiver Ge schwindigkeitsbegriff, vgl. 5. Szene in Kap. 5.1). Im Laufe der Bearbeitung kommen sie gelegentlich zum Schluss, dass es sie doch nicht gibt, weil sie diese nicht berechnen können. In diesem Sinn wird dann die m-Welt über die $\mathrm{F}$ Welt gestellt. Dominiert jedoch die r-Welt, wird das Verbiegen unterbunden: „Es gibt eine Momentangeschwindigkeit, aber wir können sie nicht ermitteln. “143

Die Erschließung von Mathematik aus außermathematischen Problemen entspricht der in Kapitel 1.1 zitierten Forderung Freudenthals. Hier kann ein Bild von Mathe matik entstehen, das sie nicht als starres Gebäude aus feststehenden Regeln und Formeln

\footnotetext{
${ }^{141}$ [vom Hofe 1995b], S. 42

142 [NRW 1999], S. 16

143 [10; 111 - 112]
} 
darstellt. Die Mathematik kann als vom Menschen geschaffenes Gedankengebäude verstanden werden, das u.a. Werkzeuge zur Problemlösung bereit stellt.

\section{Die Sichtweisen im Unterricht - ein Vorschlag}

Es bleibt die Gretchen-Frage, wie die Sichtweisen im Mathematikunterricht berücksichtigt werden könnten. In diesem Absatz möchte ich einige Ideen vorstellen.

Wenn die Sichtweisen beim Erwerb eines intuitiven Grenzwertbegriff helfen sollen, müssen sie recht früh in einer Unterrichtseinheit „Diffe renzialrechnung“ auftauchen. Wie oben angedeutet, könnte ich mir vorstellen, die Jan-Ullrich-Aufgabe aus meiner Untersuchung zu Beginn der Unterrichtsreihe bearbeiten zu lassen. Das könnte z.B. in einer Partnerarbeitsphase geschehen. Meine Erfahrungen lassen mich erwarten, dass die Sichtweisen in Form der in meiner Arbeit beschriebenen Phänomene auftauchen. Beim Vergleich der Arbeitsergebnisse zum Teil c) der Jan-Ullrich-Aufgabe sollten also Argumentationen für und gegen die Existenz der Momentangeschwindigkeit aufeinanderprallen. Sind die Positionen auf der Phänomenebene bekannt, könnte sich eine zweite Einzel- oder Partnerarbeitsphase anschließen, in der alle Schülerinnen und Schüler Argumente für beide Positionen erarbeiten. Dadurch könnte man eine weitere intensive Aus einandersetzung mit den Positionen erreichen.

Beim anschließenden Sammeln der Argumente kann eine Sortierung nach den Kriterien „Momentangeschwindigkeit gibt es.“ bzw. „Momentangeschwindigkeit gibt es nicht“, vorgenommen werden. Je nach Diskussionskompetenz und -freudigkeit des Kurses könnte die Phase des Sammelns der Argumente verschieden ablaufen:

- Die Lehrperson behält die Diskussionsleitung und sammelt die Argumente.

- Je eine Vertreterin bzw. ein Vertreter der beiden Positionen treten an die Tafel und sammeln Argumente ihrer Mitschülerinnen und -schüler für ihre bzw. seine Position.

- Die Einzel oder Partnerarbeitsphase kann auch mit der Sammelphase zusammenfallen, wenn die Schülerinnen und Schüler ihre Argumente sofort und zunächst kommentarlos an die Tafel schreiben. Der Tafelanschrieb ermöglicht es den anderen Teilnehmerinnen und Teilnehmer des Kurses, in Ruhe auf die neuen Argumente 
einzugehen. Auch dadurch kann vielleicht erreicht werden, dass sich die Schülerinnen und Schüler intensiv mit den Positionen auseinandersetzen.

- In einem schwachen Grundkurs könnten Argumentationsanstätze durch die Lehrerin bzw. den Lehrer vorgegeben werden, die zu ergänzen sind. Eine weitere Erleichterung könnte eine Sammlung möglicher Argumentationsstränge sein, die lediglich noch den Positionen zuzuordnen wären.

Anhand der Sammlung der Argumente können im späteren Verlauf die Eigenschaften der Sichtweisen abstrahiert werden. Wahrscheinlich kann der Abstraktions prozess erleichtert werden, wenn eine große Anzahl von Argumenten für beide Positionen zur Verfügung steht. Da die Sichtweisen unabhängig von der Einkleidung der Situation auftreten, könnten die Positionen z.B. auch anhand der Frage, ob eine Kurve an einer Stelle eine Steigung hat, diskutiert werden. In Kasten 1 ist die zur Untersuchungsaufgabe entsprechende „Steigungs“-Version der Aufgabe notiert. Natürlich müssten die Teilaufgaben wie in dieser Untersuchung nach und nach aufgedeckt werden, damit Aufgabenteil c) sinnvoll bleibt. Aufgrund der außermathematischen Situation in der Jan-Ullrich-Aufgabe der Untersuchung konnte das von mir als positiv empfundene Spannungsverhältnis zwischen den Denkwelten erzeugt werden, vgl. Kapitel 2.1. Die „Steigungs“-Version ist innermathematisch. Die g-Welt ist ein Teil der m-Welt. Ob mit der „Steigungs“-Version der Aufgabe das besagte Spannungsverhältnis aufgebaut werden kann, bezweifle ich. Will man dennoch an dem Steigungsgedanken festhalten, bietet sich die Idee einer Höhe-Zeit-Funktion eines Flugzeugs während eines Flugs an. Verbindet man die Steigung des Funktionsgraphs mit der Steigung des Flugzeugs und diese wiederum mit der Richtung der Längsachse des Flugzeugs (als Gerade), ist die Frage nach der Steigung des Graphs in einem Punkt fast trivial: Natürlich hat das Flugzeug zu jedem Zeitpunkt einen Steigung, die der Steigung der besagten Geraden entspricht. Die Frage nach der Steigung kann unabhängig von der Tatsache, dass die lokale Höhen-Änderung Null ist, beantwortet werden. Wie bei der Bewegungsaufgabe trennt man sich von Überlegungen bezüglich der lokalen Änderung der Funktionswerte, die der isolierenden Sichtweise zugeschrieben werden können. Die Vorstellung vom Flugzeug erleichtert diese Trennung. Genauso, wie man bei der Bewegungsaufgabe intuitiv von der Existenz einer Geschwindigkeit-Zeit-Funktion aus geht, hilft die Vorstellung vom Flugzeug, ebenso die Existenz der Steigungsfunktion (des Flugzeugs sowie bzgl. des Graphs der Höhe-Zeit-Funktion) zu akzeptieren. 


\section{Aufgabe:}

Folgende Aufgabe besteht aus fünf Teilaufgaben. Jede Teilaufgabe soll schriftlich bearbeitet werden.

a) Gegeben ist eine Funktion $f$ mit $f(t)=\frac{28 t(10-t)}{5}$ für $t \in[0 ; 5]$.

Folgende Wertetabelle gibt einige Funktionswerte an:

\begin{tabular}{|l|l|l|l|l|l|l|l}
\hline $\mathrm{t}$ & 0.5 & 1 & 1.5 & 2 & & & \\
\hline $\mathrm{f}(\mathrm{t})$ & 26.6 & 50.4 & 71.4 & 89.6 & & & \\
\hline
\end{tabular}

Berechne den Funktionswert für $\mathrm{t}=3$.

b) Nun sollen einige Durchschnittsteigungen des Funktionsgraphs von $f$ bezüglich der Stelle $\mathrm{t}=3$ berechnet werden. Berechne die Durchschnittssteigungen zwischen 0 und 3 , zwischen 0.5 und 3, zwischen 1 und 3, 1.5 und 3, 2 und 3, 2.5 und 3.

c) Bisher war immer von Durchschnittssteigung die Rede. Gibt es auch so etwas wie eine Steigung an der Stelle $\mathrm{t}=2$ ? Begründe!

d) Wie kannst Du möglichst genau die Steigung an einer Stelle berechnen?

e) Überlege, ob und wie Du mit Deiner Methode die Steigung an einer Stelle ganz genau berechnen kannst.

Kasten 1: Die Aufgabe im Gewand „Steigung einer Kurve an einer Stelle“

Natürlich kann auch die bekannte „Badewannen-Aufgabe“ herangezogen werden, bei der nach Wasser-Zulauf- und -ablaufgeschwindigkeiten gefragt wird. Eine Sammlung von Situationen für die sich entsprechende Aufgaben formulieren lassen, findet man in [Blum/Törner 1983, S. 92].

Die Sammlung von Argumenten zu den beiden Positionen anhand verschiedener Situationen (Momentangeschwindigkeit, Kurvensteigung an einer Stelle, Zuflussgeschwindigkeiten,...) kann aufzeigen, dass die Positionen unabhängig von den Phänomenen in den verschiedenen Situationen auftreten. Die Frage nach den von den konkreten Situationen unabhängigen Ausprägungen der Positionen leitet den Abstraktionsprozess von den Phänomenen zu den Sichtweisen ein. Die Schülerinnen und Schüler haben die Aufgabe, nach den von den konkreten Situationen unabhängigen Eigenschaften (das Eingebettet- und Voneinander-isoliert-Sehen) der Positionen zu suchen und zu benennen. Ein Schritt der Abstraktion besteht darin, dass den Positio- 
nen Namen gegeben werden, etwa „isolierende“ und „einbettende Sichtweise“. Anschließend sollten weitere Übungen durchgeführt werden, z.B. die Umkehrung der Denkrichtung: Man könnte von verschiedenen Situationen ausgehend die Sichtweisen unter der Fragestellung betrachten: Isolierend bzw. oder einbettend Sehen bedeutet in dieser Situation...

Es schließt sich die Frage an, welche Sichtweise zu bevorzugen ist. Wie in Kapitel 4 beschrieben, sollte die einbettende Sichtweise betont werden: Sie liegt dem Denken der Schülerinnen und Schüler nahe (vgl. Kapitel 4.1); Sie kann bei der Ermittlung des Grenzwerts helfen (vgl. Kapitel 4.2); Sie ist aus mathematischer Sicht akzeptabel (vgl. Kapitel 4.5). Diese Frage wird also durch diese Arbeit beantwortet, und nicht von den Schülerinnen und Schülern, die weder Maßtheorie noch Ergebnisse der Mathematikdidaktik kennen. Die Entscheidung für die einbettende Sichtweise als die besser passende kann durch die Lehrperson durch folgende Argumente vor dem Kurs vertreten werden: „Wenn man zu keinem Zeitpunkt Geschwindigkeit hätte, würde man sich überhaupt nicht bewegen, da man ja nie Geschwindigkeit hätte, auch über einem Zeitraum nicht." ${ }^{144}$ Da aber Bewegungen über Zeiträume stattfinden, muss es auch zu den Zeitpunkten eine Geschwindigkeit geben, obwohl zu einem Zeitpunkt keine Bewegung möglich ist. - Zenons Pfeil fliegt und wird ankommen, das ist eine Alltagserfahrung. Es wird niemanden geben, der sich freiwillig als Zielscheibe für den Pfeil zur Verfügung stellt, und das, obwohl der Pfeil zu keinem Zeitpunkt einen Weg zurücklegt. - Jan Ullrich wird vom Start bis in das Ziel fahren, auch er wird ankommen. - Eine Kurve hat in jedem Punkt eine Richtung: Hinterlässt ein Bus (ohne Gelenk in der Mitte) eine Reifenspur, kann die Richtung in jedem Punkt der Spur mit der Richtung der Längsachse des Busses an jeder Stelle identifiziert werden. Das alles sind keine Beweise für die Existenz lokaler Änderungsraten in verschiedenen Situationen. Aber sie lassen sie höchst plausibel erscheinen. Darauf stützt sich die Entscheidung, dass die einbettende Sichtweise die für die Beschreibung der Alltagswelt in der $r$-Welt adäquat ist.

Zuletzt bleibt die Frage, wie man eine lokale Änderungsrate berechnen kann, die Frage nach einem Kalkül, der die gängigen Konvention berücksichtigt. Aufgrund der

\footnotetext{
${ }^{144}$ Hier zeigt sich wieder das Grundparadoxon „Zusammensetzen von kontinuierlichen aus diskreten Größen“, vgl. Kap. 4.5.
} 
Aufgabenteile d) und e) werden wahrscheinlich einige der Schülerinnen und Schüler auf die Idee des Grenzprozesses gestoßen sein. Diese Idee kann nun aufgenommen werden. Fast automatisch stellt sich die Frage, „wie genau“ er ist. Nun können die Schülerinnen und Schüler die erarbeiteten Sichtweisen nutzen, um sowohl die Bedeutung des Grenzwerts als Wert der Momentangeschwindigkeit zu erkennen, als auch dessen Exaktheit, vgl. Kapitel 4.2.

Der Mathematikunterricht sollte meiner Meinung nach ermöglichen, Mathematik nicht als „Vom Himmel gefallene“ Wahrheit zu erleben, sondern als vom Menschen günstig gewähltes und ausgehandeltes Gedankengebäude. Hier liegt ein dazu geeignetes Beispiel vor.

Die Sichtweisen - zu schwer für den Unterricht?

Der Grenzwertbegriff stellt vielleicht den anspruchvollsten Begriff der Oberstufenmathematik dar. Gelegentlich wird die Frage gestellt, ob die Begriffe der Infinitesimalrechung für die durchschnittliche Schülerin bzw. den durchschnittlichen Schüler unter den üblichen Unterrichtsbedingungen nicht zu schwierig sei ${ }^{145}$. Wenn man diese Frage für sich positiv beantwortet, muss man sich weiter fragen, ob die Hinzunahme der Sichtweisen als eigene Begriffe statt zu einer Klärung des Grenzwertbegriffs zu einer (zusätzlichen) Überforderung führt.

An der vorliegenden Untersuchung haben Schülerinnen und Schüler der Jahrgangsstufen 10 und 12 teilgenommen. Die Schülerinnen und Schüler der Jahrgangsstufe 12 besuchten Mathematik-Grund- und -Leistungskurse. Ihre Leistungen im Unterricht erstreckten sich von „mangelhaft“ im Grundkurs bis „sehr gut“ im Leistungskurs. - Die Transkripte zeigen, dass alle Schülerinnen und Schüler in der Lage sind, die Phänomene, die zu den Sichtweisen führen können, auf eben der Phänomenebene zu diskutieren. Aus meiner Sicht unterscheidet sich das Niveau der Diskussion auf der Jahrgangsstufe 10 kaum von dem auf der Jahrgangsstufe 12. Man kann also erwarten, dass im Unterricht eine Sammlung von Argumenten für beide Sichtweisen auf der Phänomenebene erstellt werden kann. Weitere Prognosen lassen sich aufgrund der Transkripte nicht aufstellen: In meiner Untersuchung endete die Partner-

${ }^{145}$ vgl. [Bender 1991b] 
arbeitsphase oft mit dem in Kapitel 5.1 beschriebenen inneren Konflikt. Das anschließende Gespräch mit dem Versuchsleiter kann nicht als eine Art Unterrichtsgespräch aufgefasst werden und daher nicht weiterhelfen, und das schon deswegen, weil zum Zeitpunkt der Gespräche die Sichtweisen beim Versuchsleiter noch nicht ausgeschärft waren ${ }^{146}$, er also keine Hilfen, die auf den Sichtweisen basieren, hat anbieten können. Es werden wohl Unterrichtsversuche benötigt, damit die Auswirkungen der Sichtweisen auf die Begriffsbildung im Analysisunterricht eingeschätzt werden können.

\footnotetext{
${ }^{146}$ Sie entstanden schließlich erst als Deutungshypothesen bei der Interpretation der Transkripte, vgl.
} Kapitel 2.3. 


\section{Schlussbemerkung}

Bei meiner Arbeit waren mir drei grundlegende Gesichtspunkte wichtig:

- Die Schulpraxis betreffend: Die Kategorien der einbettenden und der isolierenden Sichtweise macht einen besonderen inhaltlichen Aspekt des Denkens in funktionalen Abhängigkeiten zugänglich. Sie liefern damit sowohl ein Instrument zur Analyse von Schülerinnen und Schüleräußerungen und den dahinter stehenden Denkweisen im Analysisunterricht als auch für den Entwurf für Unterrichtseinheiten insbesondere zur Einführung in die Differenzialrechnung

- Die Didaktik der Mathematik betreffend: Mit Hilfe der Kategorien der Sichtweisen konnte gezeigt werden, dass der didaktische Ansatz, die lokale Änderungsrate dem Ableitungsbegriff als Grundvorstellung zu unterlegen, in sich das Grundparadoxon infinitesimalen Denkens als geistiges Hindernis trägt, und daher in der gängigen Form nur bedingt geeignet ist. Die Auseinandersetzung mit beiden Sichtweisen und anschließende Betonung der einbettenden Sichtweise könnte dazu beitragen, das geistige Hindernis zu überwinden.

- Die Forschungsmethode betreffend: Nicht zuletzt kann diese Arbeit als Beleg für die Möglichkeit dienen, Stoffdidaktik und empirische Unterrichtsforschung gewinnbringend miteinander zu verbinden. 


\section{Literaturverzeichnis}

[Arons 1965]: Arons, Arnold B.: „Development of concepts of physics“; Addison Wesley; Massachusetts 1965

[Arons 1990]: Arons, Arnold B.: „A guide to introductory physics teaching“; Wiley;

New York 1990

[Athen/Griesel 1979]: Hermann Athen, Heinz Griesel (Hrsg.): „Mathematik heute -

Einführung in die Analysis1“; Schroedel Verlag, Hannover 1979

[Bauersfeld 1983]: Heinrich Bauersfeld: „Subjektive Erfahrungsbereiche als

Grundlage einer Interaktionstheorie des Mathematiklernens und -lehrens";

in: Heinrich Bauersfeld u.a. (Hrsg.) : „Lernen und Lehren von Mathematik“;

Aulis Verlag, Köln 1983

[Baumann 1998]: Rüdeger Baumann: „Analysis 1“; Klett Verlag; Stuttgart, 1998

[Beck/Jungwirth 1999]: Christian Beck, Helga Jungwirth: „Deutungshypothesen in der interpretativen Forschung" in: Journal für Mathematik-Didaktik (JMD), Jahrgang 20 (1999); S. $231-259$

[Becker 1975]: Oskar Becker: „Grundlagen der Mathematik in Geschichtlicher

Entwicklung“; Suhrkamp Taschenbuch Verlag; München 1975;

[Bender 1991a]: Peter Bender: „Ausbildung von Grundvorstellungen und

Grundverständnissen“; in: Helmut Postel u.a. (Hrsg.): „Mathematik lehren und lernen“; Schroedel Verlag, Hannover 1991;

[Bender 1991b]: Peter Bender: „Fehlvorstellungen und Fehlverständnisse bei Folgen

und Grenzwerten"; Der mathematische und naturwissenschaftliche

Unterricht (MNU); Jahrgang 44 (1991), S. 238 - 243

[Bivens 1986]: Irl C. Bivens: "What a Tangent Line is When it isn't a Limit“; College

Mathematics Journal; Vol 17 (Mar 1986), p. 133 - 143

[Blum 1985]: Werner Blum: „Anwendungsorientierter Mathematikunterricht in der

didaktischen Diskussion“; in: Mathematische Semesterberichte; Jahrgang

32 (1985), S. $195-232$

[Blum/Törner 1983]: Werner Blum; Günter Törner: „Didaktik der Analysis“;

Vandenhoeck \& Ruprecht; Göttingen 1983 
[Brousseau 1983]: G. Brousseau: „Les obstacles épistémologique et les problèmes en mathématiques", in: Revue Recherches en Didactique des mathématiques ; Vol. 4 (1983), p. 165 - 198

[Busse 1999]: Andreas Busse: „Mathematische Modellbildung aus der Sicht des Subjekts"; in: Der mathematische und naturwissenschaftliche Unterricht (MNU); Jahrgang 52 (1999), S. 243 - 345

[Dellian 1988]: Ed Dellian (Hrsg.): „Isaac Newton: Mathematische Grundlagen der Naturphilosophie"; Ausgewählt, übersetzt, eingeleitet und herausgegeben von Ed Dellian; Übersetzung der 3. lateinischen Ausgabe von 1726; Felix Meiner Verlag; Hamburg 1988

[Duden 1984]: „Das große Duden Schülerlexikon“; Bibliographische Institut Mannheim; Meyers Lexikonverlag; Mannheim 1984

[Fischbein u.a. 1979]: Efraim Fischbein u.a.: „The intuition of infinity“; Educational Studies in Mathematics; Vol 10 (1979), p. 3 - 40

[Freudenthal 1974]: Hans Freudenthal "Mathematik als pädagogische Aufgabe Bd.1“; Klett Verlag; Stuttgart 1974

[Glaser/Scheid/Wellstein 1983]: Herbert Glaser, Harald Scheid, Hartmut Wellstein (Hrsg.): „, Grundkurs Analysis“; Klett Verlag; Stuttgart 1983

[Glaser/Strauss 1979a]: Barney G. Glaser; Anselm L. Strauss: „Die Entdeckung gegenstandsbezogener Theorie: Eine Grundstrategie qualitativer Sozialforschung“; in: Christel Hopf (Hrsg.): „Qualitative Sozialforschung“ Klett-Cotta Verlag; Stuttgart 1979; S. 91 - 111

[Glaser/Strauss 1979b]: Barney G. Glaser; Anselm L. Strauss: „Die Entdeckung begründeter Theorie“; in: Klaus Gerdes (Hrsg.): „Explorative Sozialforschung“: Ferdinand Enke Verlag; Stuttgart 1979; S. 63 - 67

[Griesel 1976]: Heinz Griesel: „Grundkurs Analysis - die Beschreibung des Ablaufs einer Curriculumentwicklung“; Mathematikunterricht (MU); Jahrgang 22 (1976), S. $25-46$

[Griesel/Postel 1999]: Heinz Griesel, Helmut Postel (Hrsg.): „Elemente der

Mathematik 11. Schuljahr Nordrhein-Westfalen“; Schroedel Verlag; Hannover 1999

[Hahn/Dzewas 1994]: Jutta Cukrowicz (Hrsg.): „Mathematik Grundkurs Analysis Gesamtband“; Westermann Verlag; Braunschweig 1994 
[Hefendehl-Hebeker 1989]: Lisa Hefendehl-Hebeker: „Die negativen Zahlen zwischen anschaulicher Deutung und gedanklicher Konstruktion - geistige Hindernisse in ihrer Geschichte“, in: mathematik lehren; Heft 35, S. 6 - 12 [Hering 1989]: Hermann Hering: „Begriffsentwicklung und präformales Beweisen bei infinitesimalen Prozessen“; Journal für Mathematikdidaktik (JMD); Jahrgang 10 (1989), S. 123 - 140

[Heuser 1990]: Harro Heuser: „Lehrbuch der Analysis Teil I“; Teubner Verlag;

Stuttgart, 1990

[Hölzl 1994]: Reinhard Hölzl: „Im Zugmodus der Cabri-Geometrie“; Deutscher

Studien Verlag; Weinheim 1994

[Jahnke 1998]: Thomas Jahnke: „Zur Kritik und Bedeutung der Stoffdidaktik“;

mathematica didactica; Jahrgang 21 (1998), S. 61- 74

[Kirsch 1960]: Arnold Kirsch: „Ein geometrischer Zugang zu den Grundbegriffen der Differentialrechnung“; Mathematikunterricht (MU); Jahrgang 6 (1960), S. 5 $-21$

[Kroll 1985]: Wolfgang Kroll: „Grund- und Leistungskurs Analysis Band 1:

Differentialrechnung“; Dümmler Verlag; Bonn 1985

[Malle 1988]: Günther Malle: „Entstehung neuer Denkgegenstände“; in: Willibald

Dörfler: „Kognitive Aspekte mathematischer Begriffsbildung“; Hölder-

Pichler-Tempsky; Wien 1988, S. 259 - 319

[Malle 1993]: Günther Malle: „Didaktische Probleme der elementaren Algebra“;

Vieweg Verlag; Wiesbaden 1993

[NRW 1981]: Kultusminister des Landes Nordrhein-Westfalen (Hrsg.) „Richtlinien für die gymnasiale Oberstufe in Nordrhein-Westfalen, Mathematik“; Greven Verlag; Köln 1981

[NRW 1999]: Ministerium für Schule und Weiterbildung, Wissenschaft und Forschung des Landes Nordrhein-Westfalens (Hrsg.): „Richtlinien und Lehrpläne für die Sekundarstufe II - Gymnasium / Gesamtschule in NordrheinWestfalen“; Ritterbach Verlag; Frechen 1999

[Oevermann 1983]: Ulrich Oevermann, u.a.: „Die Methodologie einer ,Objektiven

Hermeneutik'“, in: Peter Zedler, Heinz Moser (Hrsg.): „Aspekte qualitativer

Sozialforschung“, Leske + Budrich, Oplanden 1983

[Schmid/Schweizer]: August Schmid, Wilhelm Schweizer (Hrsg.): „Analysis

Leistungskurs Gesamtausgabe“; Klett Verlag; Stuttgart 1990 
[Sierpinska 1992]: Anna Sierpinska: „On understanding the notion of function“ in:

Guershon Harel, Ed Dubinsky (Hrsg.): „The concept of function - Aspects of epistemology and pedagogy“; American Mathematical Society Notes, Volume 25 (1992), p. 25 - 58

[Stachowiak 1973]: Herbert Stachowiak: „Allgemeine Modellbildungstheorie“;

Springer Verlag; Wien 1973

[Voigt 1984]: Jörg Voigt: „Interaktionsmuster und Routinen im Mathematikunterricht“;

Beltz Verlag, Weinheim 1984

[Voigt 1996]: Jörg Voigt: „Empirische Unterrichtsforschung in der Mathematikdidak-

tik“, in: Gerd Kadunz u.a.(Hrsg.): „Trends und Perspektiven“; Schriften-

reihe Didaktik der Mathematik Bd. 23, Hölder-Pichler-Tempsky, Wien 1996

[Volkert 1988]: Volkert, Klaus: „Geschichte der Analysis“; BI Wissenschaftsverlag;

Mannheim 1988

[Vollrath 1989]: Hans-Joachim Vollrath: „Funktionales Denken“; Journal für

Mathematikdidaktik (JMD); Jahrgang 10 (1989), S. 3 - 37

[Vollrath 1994]: Hans-Joachim Vollrath: „Algebra in der Sekundarstufe“; Bト

Wissenschaftsverlag; Mannheim 1994

[vom Hofe 1995a]: Rudolf vom Hofe: „Grundvorstellungen mathematischer Inhalte“;

Spektrum Verlag; Heidelberg 1995

[vom Hofe 1995b]: Rudolf vom Hofe: „Vorschläge zur Öffnung normativer

Grundvorstellungskonzepte für deskriptive Arbeitsweisen in der

Mathematikdidaktik“; in: Hans-Georg Steiner / Hans-Joachim Vollrath

(Hrsg): „Neue problem- und praxisbezogene Forschungsansätze“; Aulis

Verlag; Köln 1995

[vom Hofe 1996]: Rudolf vom Hofe: „Über die Ursprünge des Grundvorstellungs-

konzepts in der deutschen Mathematikdidaktik“ Journal für Mathematik-

Didaktik (JMD); Jahrgang 17 (1996), S. 238-264.

[vom Hofe 1998]: Rudolf vom Hofe: „Probleme mit dem Grenzwert - genetische

Begriffsbildung und geistige Hindernisse“ Journal für Mathematik-Didaktik (JMD); Jahrgang 19 (1998), S. 257 - 291

[vom Hofe 1999]: Rudolf vom Hofe: „Explorativer Umgang mit Funktionen“; Journal

für Mathematik-Didaktik (JMD); Jahrgang 20 (1999), S. 186 - 221 
[Weigand 1988]: Hans-Georg Weigand: „Zur Bedeutung von Zeitfunktionen für den

Mathematikunterricht“; Journal für Mathematik-Didaktik (JMD); Jahrgang 9 (1988), S. 55 - 86

[Weigand 1993]: Hans-Georg Weigand: „Zur Didaktik des Folgenbegriffs“; BI

Wissenschaftsverlag; Mannheim 1993

[Weyl 1921]: Hermann Weyl: „Über die neue Grundlagenkrise der Mathematik“;

Mathematische Zeitschrift, Band10, S. 39 - 79; Springer Verlag, Berlin 1921

[Wolfers 1872]: J. Ph. Wolfers: „Isaac Newton: Mathematische Prinzipien der

Naturlehre“; mit Bermerkungen und Erläuterungen und herausgegeben von J. Ph. Wolfers; unveränderter Nachdruck der Ausgabe Berlin 1872; wahrscheinlich Übersetzung der 3. lateinischen Ausgabe, da ein Vorwort zur 3. Ausgabe vorhanden ist; Wiss. Buchgesellschaft; Darmstadt 1963 


\section{Anhang}

\section{Verzeichnis der Transkripte}

Die Transkripte sind Abschriften der von mir videographierten Arbeits- und Gesprächsphasen der Schülerinnen und Schüler, die die von mir gestellte Aufgabe bearbeiteten. Die Videoaufnahmen wurden nach der zeitlichen Reihenfolge der Aufnahmen von 01 bis 16 durchnumeriert. Die Dialoge und die Beschreibungen der Handlungen zwischen zwei Gesprächsabschnitten wurden zeilenweise nummeriert. Somit sind z.B. mit [03; 23-25] die Zeilen 23 bis 25 des dritten Transkripts gemeint.

Alle vollständigen Transkripte befinden sich auf der beiliegenden CD-ROM.

[01]: Hüseyin und Tobias; Jahrgangsstufe 11.2 LK

[02]: Anika und Juliane; Jahrgangsstufe 10

[03]: Christian und Heiko; Jahrgangsstufe 10

[04]: Annika und Sandra; Jahrgangsstufe 12 LK

[05]: Heike und Kathrin ; Jahrgangsstufe 12 GK

[06]: Christine und Kathrin; Jahrgangsstufe 12 GK

[07]: Sven und Thomas; Jahrgangsstufe $12 \mathrm{GK}$

[08]: Anne und Petra; Jahrgangsstufe 12 GK

[09]: Barbara und Isabelle; Jahrgangsstufe 12 GK

[10]: Alexandra und Alexandra; Jahrgangsstufe 12 GK

[11]: Angela und Christina; Jahrgangsstufe $12 \mathrm{GK}$

[12]: Maren und Nadia; Jahrgangsstufe 12 GK

[13]: Alexander und Sebastian; Jahrgangsstufe 12 LK

[14]: Claudia und David; Jahrgangsstufe 12 LK

[15]: Ann-Kathrin und Julia; Jahrgangsstufe 12 GK

[16]: Haug und Mareike; Jahrgangsstufe 12 LK 


\section{Abbildungsverzeichnis}

Abbildung 1: Newtons Funktionsbegriff mit Indivisibilien, Fluenten und Fluxionen.... 8

Abbildung 2: Skizze der zentralen Begrifflichkeiten in den Kapiteln 3 und 4 ... 17

Abbildung 3 : Skizze aus Transkript 11, Bearbeitung zu Aufgabenteil c) 52

Abbildung 4: Identifikation von Weglänge und Momentangeschwindigkeit 56

Abbildung 5: Ein Drehspulmessinstrument erzeugt Wechselstrom............................61

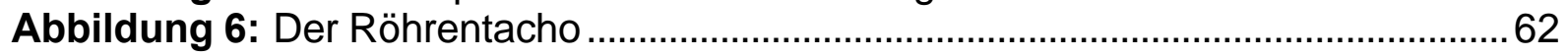

Abbildung 7: Die doppelte Einbettung von Lückenwerten......................................... 71

Abbildung 8: Der Graph von $\mathrm{f}$, eingeschlossen in eine „Parabelschere“ aus $\mathrm{x} \mapsto \mathrm{x}^{2}$ und $x \mapsto-x^{2}$

Abbildung 9: Zwei alternative Antworten zu Aufgabenteil c ....................................97

Abbildung 10: Die Bearbeitung von Aufgabenteil c) ..............................................119

Abbildung 11: D's Treppenfunktion ......................................................................122

Abbildung 12: Tangenten an Parabeln und Kreisen...............................................131

Abbildung 13: Erweiterung des Tangentenbegriffs ..............................................132

Abbildung 14: Der Zusammenhang von Ableitung und Tangentensteigung ...........132

Abbildung 15: Der Grenzwert stetiger Funktionen als Lückenwert ...........................133

Abbildung 16: Der Wert der Tangentensteigung als Lückenwert..............................134

Abbildung 17: Der Wert der Ableitung als Momentangeschwindigkeit.....................135

\section{Verzeichnis der Tabellen}

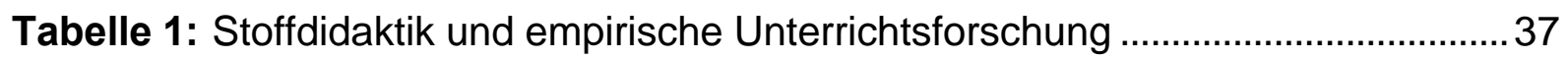

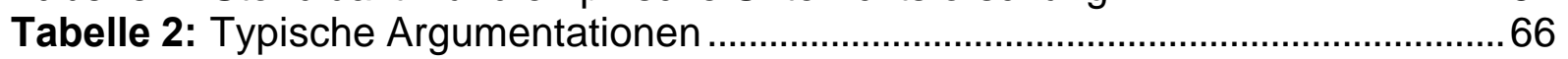

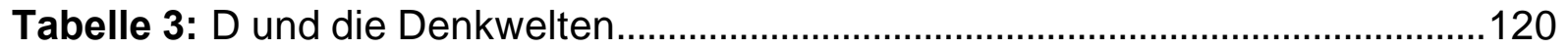

\section{CD-ROM}

Auf der CD-ROM befindet sich die Dissertationsschrift sowie alle Transkripte als .pdfDateien. Starten der CD-ROM mit X:lindex.html ( $X$ = Laufwerksbuchstabe des CDROM-Laufwerks). Von der CD-ROM kann zudem den AcrobatReader 4.0 .5 für Windows, Macintosh und LINUX installieren.

Tim Rinkens hat die kleine Fahrrad-Animation zur einbettenden Sichtweise programmiert. 